\title{
COMPUTATIONAL AND EXPERIMENTAL MODELING OF SLURRY BUBBLE COLUMN REACTORS
}

\author{
Grant No.: DE-FG-98FT40117 \\ U.S. Department of Energy \\ National Energy Technology Laboratory \\ University Coal Research \\ Program Manager: Dr. Donald Krastman
}

FINAL TECHNICAL REPORT

\author{
Dr. Paul C. K. Lam \\ Principal Investigator (2000-2002) \\ Associate Dean, College of Engineering \\ The University of Akron \\ Akron, Ohio 44326-3903 \\ Tel.: 330-972-7930; Fax: 330-972-5162 \\ Email: plam@uakron.edu \\ Dr. Isaac K. Gamwo \\ Principal Investigator (1998-1999) \\ Department of Mechanical Engineering \\ The University of Akron \\ Akron, Ohio 44326-3906 \\ and \\ Dr. Dimitri Gidaspow \\ Co-Principal Investigator \\ Distinguished Professor \\ Department of Chemical and Environmental Engineering \\ Illinois Institute of Technology \\ Chicago, IL 60616 \\ Tel.: 312-567-3045; Fax: 312-567-8874 \\ Email: gidaspow@iit.edu \\ INDUSTRY COLLABORATORS: \\ Dr. Bing Sun, UOP, Des Plaines, IL 60017 \\ Dr. Rachid Oukaci, Energy International Corporation, Pittsburgh, Pennsylvania \\ Award Period \\ September 1, 1998 - July 30, $2001+8$ months no cost extension
}

May 2002 


\title{
COMPUTATIONAL AND EXPERIMENTAL MODELING OF SLURRY BUBBLE COLUMN REACTORS
}

\begin{abstract}
The objective of this study was to develop a predictive experimentally verified computational fluid dynamics (CFD) model for gas-liquid-solid flow. A three dimensional transient computer code for the coupled Navier-Stokes equations for each phase was developed and is appended in this report. The principal input into the model is the viscosity of the particulate phase which was determined from a measurement of the random kinetic energy of the 800 micron glass beads and a Brookfield viscometer. The details are presented in the attached paper titled "CFD Simulation of Flow and Turbulence in a Slurry Bubble Column". This phase of the work is in press in a refereed journal (AIChE Journal, 2002) and was presented at the Fourth International Conference on Multiphase Flow (ICMF 2001) in New Orleans, May 27-June 1, 2001 (Paper No.
\end{abstract} 909).

The computed time averaged particle velocities and concentrations agree with Particle Image Velocimetry (PIV) measurements of velocities and concentrations, obtained using a combination of gamma-ray and X-ray densitometers, in a slurry bubble column, operated in the bubbly-coalesced fluidization regime with continuos flow of water. Both the experiment and the simulation show a down-flow of particles in the center of the column and up-flow near the walls and nearly uniform particle concentration.

Normal and shear Reynolds stresses were constructed from the computed instantaneous particle velocities. The PIV measurement and the simulation produced instantaneous particle velocities. The PIV measurement and the simulation produced similar nearly flat horizontal profiles of turbulent kinetic energy of particles. To better understand turbulence we studied fluidization in a liquid-solid bed. This work was also presented at the Fourth International Conference on Multiphase Flow (ICMF 2001, Paper No. 910).

To understand turbulence in risers, measurements were done in the IIT riser with 530 micron glass beads using a PIV technique. This report summarizes the measurements and simulations completed so far. This work will continue under the sponsorship of the National Science Foundation and Dow Corning Corporation. This phase of the work is part of the DOE/Industry/University Multiphase Fluid Dynamics Research Consortium.

Optimization of the LaPorte pilot plant reactor was attempted by rearranging the heat exchangers. The paper accepted for presentation at the Sixth World Congress of Chemical Engineering, Melbourne, Australia, September 23-27, 2001 is a part of this report. 


\section{Disclaimer}

This report was prepared as an account of work sponsored by an agency of the United States Government. Neither the United States Government nor any agency therefor, nor any of their employees, makes any warranty, express or implied, or assumes any legal liability or responsibility for the accuracy, completeness, or usefulness of any information, apparatus, product, or process disclosed, or represents that its use would not infringe privately owned rights. Reference herein to any specific commercial product, process, or service by trade name, trademark, manufacturer, or otherwise does not necessarily constitute or imply its endorsement, recommendation or favoring by the United States Government or any agency thereof. The views and opinions of authors expressed herein do not necessarily state or reflect those of the United States Government or any agency thereof. 


\section{Table of Contents}

Executive Summary

Objective

Accomplishments

CFD Simulation of Flow and Turbulence in a Slurry Bubble Column (in press AIChE Journal, 2002)

Computation of Turbulence in Liquid-Solid Reactors

Measurement and Computation of Turbulence in Risers

CFD Models for Slurry Bubble Column Reactors

Measurement of Thermal Conductivity of FCC Particles

Large Scale Oscillations or Gravity Waves in Risers and Bubbling Beds

Turbulence of Particles in a CFB and Slurry Bubble Columns Using Kinetic Theory

Articles and Presentations

Appendix: Three Dimensional Transient CFD Code 


\section{EXECUTIVE SUMMARY}

\section{OBJECTIVE}

This project was a collaborative effort between two universities (The University of Akron and Illinois Institute of Technology) and two industries (UOP and Energy International). The overall objective of this research was to develop predictive hydrodynamic models for gas-liquid-solid catalyst reactors using computational fluid dynamics (CFD) techniques. The work plan involved a combination of computational, experimental and theoretical studies with a feedback mechanism to correct model deficiencies. The tasks involved: 1- Development of a CFD code for slurry bubble column reactors. 2- Optimization. 3- Comparison to reactor data. 4- Development of a three dimensional transient CFD code. 5- Measurement of particle turbulent properties. 6a) Measurement of thermal conductivity of particles in the IIT two story riser. b) Measurements of evaporation rates of liquid nitrogen in the IIT riser.

\section{ACCOMPLISHMENTS}

Our paper describing the basic approach using kinetic theory to predict the turbulence of catalyst particles in a slurry bubble column reactor, has been published in a refereed journal (Wu and Gidaspow, 2000). The computed slurry height, gas hold up and rate of methanol production agreed with the Department of Energy La Porte pilot plant reactor data.

A three dimensional transient computer code for the coupled Navier-Stokes equations for each phase was developed and is appended in this report. The principal input into the model is the viscosity of the particulate phase which was determined from a measurement of the random kinetic energy of the 800 micron glass beads and a Brookfield viscometer. The details are presented in the attached paper titled "CFD Simulation of Flow and Turbulence in a Slurry Bubble Column". This phase of the work is in press in a refereed journal (AIChE Journal, 2002) and was presented at the Fourth International Conference on Multiphase Flow (ICMF 2001) in New Orleans, May 27 June 1, 2001 (Paper No. 909).

We have invented an alternate technique for computing turbulence in a slurry bubble column. It involves direct numerical simulation of the equations of motion with the measured particular viscosity as an input. We have computed the flow profiles, particle concentration profiles and Reynolds stresses for an IIT slurry bubble column. The computed time averaged particle velocities and concentrations agree with PIV measurements of velocities and concentrations, obtained using a combination of gammaray and X-ray densitometers, in a slurry bubble column, operated in the bubbly-coalesced fluidization regime with continuos flow of water. Both the experiment and the simulation show a down-flow of particles in the center of the column and up-flow near the walls and nearly uniform particle concentration. The computations were done using our previous two dimensional three phase code and a newly developed three dimensional version. This work was reported in the Ph.D. thesis by Diana Matonis (IIT, 2000) and in a paper submitted for publication and presentation in ICMF 2001. 
Measurements of thermal conductivity of catalyst particles in the IIT riser were completed. The details are presented in the attached manuscript titled "Measurement of Thermal Conductivity of FCC Particles". The IIT riser was redesigned to eliminate asymmetries, similarly to the Sandia National Laboratory riser, sponsored by the Multiphase Fluid Dynamics Research Consortium. Our CCD camera system was used to measure Reynolds stresses and granular temperature for $450 \mu \mathrm{m}$ glass beads. The granular temperature when computed, following the gravity wave, shows a maximum in the center, in agreement with conventional theory of granular flow. Numerical simulation of the riser is in progress.

In view of the complexity of the simulation a better understanding of the processes using simplified analytical solutions is required. Such analytical solutions are presented in the attached paper titled "Large Scale Oscillations or Gravity Waves in Risers and Bubbling Beds". This paper presents analytical solutions for bubbling frequencies and standing wave flow patterns. The flow patterns in operating slurry bubble column reactors are not optimum. They involve upflow in the center and downflow at the walls.

To obtain an optimum size for slurry bubble column reactors, we are following up on the observation of George Cody of Exxon who reported a maximum granular temperature (random particle kinetic energy) for a particle size of 90 microns. The attached paper titled "Turbulence of Particles in a CFB and Slurry Bubble Columns Using Kinetic Theory", supports George Cody's observations. However, our explanation for the existence of the maximum in granular temperature differs from that proposed by George Cody. This work was preprinted in the Fluidization and Fluid-Particle Systems Preprint Volume, AIChE, pp. 261-266. Further computer simulations and experiments involving measurements of granular temperature are needed to obtain a sound theoretical explanation for the possible existence of an optimum catalyst size.

In an attempt to optimize the system, flow patterns for the production of methanol in slurry bubble columns were investigated by rearranging the location of the heat exchangers in the La Porte pilot plant. 


\title{
CFD SIMULATION OF FLOW AND TURBULENCE IN A SLURRY BUBBLE COLUMN
}

\author{
Diana Matonis, Dimitri Gidaspow and Mitra Bahary \\ Department of Chemical and Environmental Engineering \\ Illinois Institute of Technology, Chicago, IL 60616
}

\begin{abstract}
The objective of this study was to develop a predictive experimentally verified computational fluid dynamic ( CFD ) model for gas-liquid-solid flow. A three dimensional transient computer code for the coupled Navier-Stokes equations for each phase was developed. The principal input into the model is the viscosity of the particulate phase, which was determined from a measurement of the random kinetic energy of the 800-micron glass beads and a Brookfield viscometer.

The computed time averaged particle velocities and concentrations agree with PIV measurements of velocities and concentrations, obtained using a combination of gammaray and X-ray densitometry, in a slurry bubble column, operated in the bubbly-coalesced fluidization regime with continuous flow of water. Both the experiment and the simulation show a down-flow of particles in the center of the column and up-flow near the walls and nearly uniform particle concentration.

Normal and shear Reynolds stresses were constructed from the computed instantaneous particle velocities. The PIV measurement and the simulation produced similar nearly flat horizontal profiles of turbulent kinetic energy of particles.
\end{abstract}




\section{INTRODUCTION}

Fluidized beds are widely used industrially because the particles can be introduced into and out of the reactor as a fluid and because of good heat and mass transfer in the reactor. For conversion of synthesis gas into methanol or hydrocarbon liquid fuels, a slurry bubble column reactor has several advantages over a fixed bed reactor (Bechtel Group, 1990; Viking Systems International, 1994). Cooling surface requirement is less than in a fixed bed reactor. Catalyst deactivation due to carbon formation can be handled by catalyst withdrawal and removal, whereas replacement of fixed bed catalyst requires a shutdown. In view of these advantages, slurry bubble column reactors have recently (Parkinson, 1997;) become competitive with fixed bed reactors for converting synthesis gas into liquid fuels. Fan (1989) has reviewed other applications of three-phase fluidization. Slurry reactor design is usually done (Bechtel Group, 1999; Viking Systems International, 1994) using hold-up correlations. In the early nineties Tarmy and Coulaloglu (1992) of EXXON showed that there were no three phase hydrodynamic models in the literature and that there was a need for such models, as illustrated by the development of a three phase hydrodynamic model at EXXON presented at 1996 Computational Fluid Dynamics in Reaction Engineering Conference (Heard et al., 1996). Today, Computational Fluid Dynamics (CFD) has emerged as a new paradigm for modeling multiphase flow and fluidization, as seen from recent conferences (NICHE, 2000; FLUIDIZATION IX, 1998; CFD in Reaction Engineering, 2000), the formation of an industry-led, Department of Energy Multiphase Fluid Dynamics Research Consortium (Thompson, 1999), which consists of 6 national laboratories, 6 universities and American chemical companies, and papers published throughout the world.

The term CFD has come to denote minimal simulation using Navier-Stokes equations.

Three types of CFD models are being used in the literature to model gas-solid multiphase flow and fluidization:

1.Viscosity Input Models, where the principal input is an empirical viscosity. Examples are the papers of Anderson, Sundaresan and Jackson (1995) for bubbling beds, Tsuo and Gidaspow (1990) and Benyahia, Arastoopour and Knowlton (1998) for risers.

2.Kinetic Theory Based Models, as described in Gidaspow (1994).

The most successful example of this model is the prediction of the core-annular regime by Sinclair and Jackson (1989) for steady developed flow in a riser. Transient simulations and comparisons to data were done by Samuelsberg and Hjertager (1996) and Mathiesen, et al (2000) for multisize flow.

3.K-Epsilon Model., where the K corresponds to the granular temperature equation and epsilon is a dissipation for which another conservation law is required. Its success has been to model turbulence for steady single-phase flow. It appears as an option in most commercial CFD codes. Kashiwa and VanderHeyden (1998) are extending this model to multiphase flow as a part of the Multiphase Fluid Dynamics Consortium, where a discussion has begun at the quarterly meetings concerning mechanisms of turbulence production and dissipation.

In single-phase flow, the most fundamental approach to turbulence is DNS, Direct Numerical Simulation of the Navier-Stokes equations. It was quite successful in 
predicting the logarithmic velocity profile for channel flow (Kim, el al 1985) and other turbulence profiles, but with present computers and solution methods is restricted to relatively low Reynolds numbers, about 10,000. The viscosity input model for multiphase flow is a method similar to the DNS in single phase flow. With particular input viscosities, a system of coupled Navier-Stokes equations is solved producing instantaneous fluctuating velocities. Averaging of these velocities produces the normal and the shear Reynolds stresses for the various phases. Such a computation was recently done for a bubble column by Pan, Dudukovic and Chung (2000) using the Los Alamos CFDLIB code. Their comparison to the Particle Image Velocity (PIV) data of Mudde, et al (1997) was quite good.

This paper presents a similar computation for three phases. The computed time average particle gas and solids hold-ups and the particle velocities generally agree with the measurements in a slurry bubble column. The computed horizontal profile of particle turbulent kinetic energy also agrees with the PIV measurements, similar to those of Mudde, et al (1997).

Recently Pfleger, et al (1999) and Krishna, et al (1999) applied the commercial CFX code to bubble columns using the k-epsilon model, while Grevskott, et al (1996) successfully compared their computed steady state velocity profiles to their experiments. Li, et al (1999) computed the bubble shape in the three-phase system by using an advection equation for the bubble surface. Discrete particle methods have also been used for simulating gas-solid systems (e.g. Xu and Yu, 1997; Kwaguchi, Tanaka and Tsuji, 1998) but have not been applied to slurry bubble columns.

The experiments and the CFD computations presented in this paper are in the socalled bubbly coalesced flow regime, also called the churn-turbulent regime. Such a flow regime occurs in large diameter columns for reasonably large input gas velocities, say $0.02 \mathrm{~m} / \mathrm{s}$. Much below this velocity one would be in the dispersed bubble regime.

The large "bubbles" in the bubbly coalesced regime occur due to the intersection of paths of the fine bubbles. In the slurry bubble column, unlike in the bubble column without solids, these bubbles are not very distinct. They contain solids and almost disappear at high liquid velocities. Therefore they are hard to characterize experimentally and proved to be, so far, impossible to compute. The computations only show the ghosts of the bubbles, e.g. high velocities in the region where a large bubble was expected. Figure 6.3 in Gidaspow's book (1994) shows a picture of the most distinct bubbles that could be obtained in the experimental set-up described here. These bubbles are for liquid velocities about half of those analyzed in this paper.

In the CFD simulation separate momentum balances are solved for the continuous flowing liquid phase and for the discrete solid and gas phases. The gas phase is treated as a particulate phase, as is the standard practice in the CFD simulation of gas-liquid bubble columns (e.g. Pan, et al 2000 ). Since the flow inside the column is not far from homogeneous, one can add the three momentum equations and see that the total pressure is the sum of the individual phase pressures and that the mixture viscosity is the sum of the three phase viscosities. The viscosity of the bubble gas phase and its collisional pressure are small. The viscosity of the solid phase is large. Its measured value is used here. 


\section{PART I. EXPERIMENTAL BUBBLY COALESCED FLOW REGIME}

A. Experimental Setup. The setup used in the bubbly coalesced regime for volume fraction, velocity and viscosity measurement experiments consisted of four major parts: fluidization equipment, densitometers assembly, a high resolution micro-imaging I measuring system or a video-digital camera unit, and a Brookfield viscometer. A schematic diagram of the fluidized bed and video-digital camera unit for velocity measurements is shown in Figure 1. The schematic diagram for source-detector-recorder assembly for X-ray and ã-ray densitometers for volume fraction measurements is shown schematically in Figure 2.

B. Fluidization Equipment. A rectangular bed was constructed from transparent acrylic (Plexiglas) sheets to facilitate visual observation and video recording of the bed operations such as gas bubbling and coalescence, and the mixing and segregation of solids. The bed height was $213.36 \mathrm{~cm}$ and cross-section was $30.48 \mathrm{~cm}$ by $5.08 \mathrm{~cm}$. A centrifugal pump was connected to the bottom of the bed by a 1.0-inch $(2.54 \mathrm{~cm})$ diameter stainless steel pipe. Gas injection nozzles from an air compressor were connected to the sides of the bed. Liquid was stored in and recycled back to a fifty-five gallon storage tank.

The liquid and gas distributors were located at the bottom of the bed. Two perforated Plexiglas plates with $0.28 \mathrm{~cm}$ diameter holes distributed the liquid. They were placed at $35.6 \mathrm{~cm}$ and $50.8 \mathrm{~cm}$ above the bottom of the bed, with $0.25 \mathrm{~cm}$ size glass bead particles filled inside. The gas distributor consisted of six staggered porous tubes of $15.24 \mathrm{~cm}$ length and $0.28 \mathrm{~cm}$ diameter. The fine pores of porous tubes had mean diameter of 42ìm. The porous tubes were placed at the bottom of the bed just below the top liquid distributor plate.

C. Densitometer Assembly. Two densitometers were used alternatively for measuring the time-averaged volume fractions of three phases at a designated location by means of the X-ray and ã-ray adsorption techniques. The assembly consisted of radioactive sources as well as detecting and recording devices and a positioning table. A schematic diagram of the source, detector and recording devices assembly is shown in Figure 2.

(1)Radioactive Source. The source is a $200-\mathrm{mCi} \mathrm{Cu}-244$ source having 17.8-year half-life. It emitted X-rays with photon energy between 12 and $23 \mathrm{keV}$. The source was contained in ceramic enamel, recessed into a stainless steel support with a tungsten alloy packing, and sealed in welded Monel Capsule. The device had brazed Beryllium window. For the ã-ray densitometer, a 20-mCi Cs-137 source having a single y-ray of $667 \mathrm{keV}$ and a half-life of 30 years was used. The source was sealed in a welded, stainless steel capsule. The source holder was welded, filled with lead, and provided with a shutter to turn off the source. This is the same unit used previously by Seo andGidaspow (1987). 
(2)Detecting and Recording Devices. The intensity of the X-ray beam was measured by using a $\mathrm{NaI}$ crystal scintillation detector (Teledyne, ST-82-I/B). It consisted of a 2-mm thick, $5.08 \mathrm{~cm}$ diameter tube with $0.13-\mathrm{mm}$ thick Beryllium window. For ã-ray densitometer, the intensity of the ã-ray beam was detected by another NaI crystal detector (Teledyne, S-44-I/2). The dimensions of the crystal were as follows: $5.08 \mathrm{~cm}$ thick and $5.08 \mathrm{~cm}$ in diameter. The two detectors could be switched for use with different sources. The photomultiplier of the detector was connected sequentially to a preamplifier, an amplifier and a single-channel analyzer, a rate meter, and a 186 IBM compatible personal computer. The rate meter has a selector and a $0-100-\mathrm{mV}$ scale range.

(3)Positioning Table. Both the source holder and detector were affixed to either side of the bed on a movable frame and could be moved anywhere up-or-down or to-and-fro by means of an electric motor.

D. Particle Image Velocity (PIV) System. The digital camera technique used to measure particle velocities as shown on Figure 1 comprised of the following units:

1) Image Recording and Displaying Devices. A high resolution color video camera equipped with electronic shutter speed settings ranging from OFF to $1 / 10000 \mathrm{sec}$ and super fine pitch color monitor were used to record and display solid velocities.

2) Data Recording Device. A 486 I 33 MHz IBM compatible personal computer with a micro-imaging board inside and a micro-imaging software. Image-Pro Plus were used to record and store raw solid velocities data at any given location inside the fluidized bed.

E. Brookfield Viscometer. Brookfield digital viscometer (model LVDV-II+) with spring a torque of 673.7 dyne-cm was used to measure the effective bed viscosities. This viscometer can produce twenty different rotational speeds ranging from 0 to 100 revolutions per minute (rpm) at four different modes, namely, LV, RV, HA, and HBDVII+.

\section{Experimental Procedure and Interpretation.}

A. Fluidization Experiments. The liquid from the storage tank was fed to the bed from the bottom of the bed using the centrifugal pump. The gas was fed to the bed through a compressor. Both gas and liquid from the top of the bed were directed through three openings of 1.0-inch $(2.54 \mathrm{~cm})$ diameter back to the storage tank, where the gas was separated from the liquid.

In order to achieve a uniform fluidization, the liquid distributor section was designed in such a way that the pressure drop through the distributor section was $10-20 \%$ of the total bed pressure drop. The gas was distributed in the fluidized bed through the six staggered porous tubes.

Air and water were used as the gas and liquid, respectively, in this experiment. Ballotini (leaded glass beads) with an average diameter of $0.889 \mathrm{~cm}$ and a density of 2.94 
$\mathrm{g} / \mathrm{cm}^{3}$ were used as the solids. The experimental operating conditions are shown in the Table 1 (Bahary, 1994).

B. Volume Fractions Determination. X-ray and ã-ray densitometers have been used to measure porosities of fluidized beds (Miller and Gidaspow, 1992: Seo and Gidaspow, 1987; Gidaspow, et al, 1995) and solids concentrations in nonaqueous suspensions (Jayaswal, et al, 1990). These techniques are based on the fact that the liquid, gas and solid phases under consideration have different absorptivities for X-ray and ã-rays. The same concept was adopted to measure concentration profiles inside our three phase fluidization systems. The particles used in this system contain lead, to aid in the absorption of X-rays.

The intensity of the transmitted X-rays or ã-rays are described as a linear function of the volume fractions of liquid, gas and the solid phases. The amount of radiation that is absorbed by a material can be given by the Beer-Bougert-Lambert Law:

$$
\mathrm{I}=\mathrm{I}_{\mathrm{o}} \exp (-\kappa \rho l)
$$

where $\mathrm{I}$ is the intensity of transmitted radiation, $\mathrm{I}_{\mathrm{o}}$ is the intensity of incident radiation, $\hat{e}$ is the attenuation coefficient, $\tilde{n}$ is the density of material, and 1 is the path length.

The logarithmic form of equation 1 for three-phase (gas-liquid-solid) fluidized beds is

$$
\ln \left[\frac{I}{I_{o}}\right]=A_{g} \varepsilon_{g}+A_{l} \varepsilon_{l}+A_{s} \varepsilon_{s}
$$

where

$$
A=\kappa \rho l
$$

and where I is the intensity readings of the x-ray or ã-ray densitometers; and $\mathrm{a}_{\mathrm{g}}$, $\mathrm{a}_{1}$ and $\AA_{s}$ are the volume fractions of gas, liquid, and solid phases, respectively. The relation for volume fractions is:

$$
\varepsilon_{g}+\varepsilon_{l}+\varepsilon_{s}=1.0
$$

The coefficients in equations (2) were calculated using the least square error technique from the calibration measurements of the intensity readings of X-ray and ã-ray densitometers at known concentrations of gas, liquid and solids in three phase mixtures. However, these coefficients were found to have values with $20 \%$ of error for X-ray and $2 \%$ of error for ã-ray.

C. Velocity Measurements. In order to get a good visualization of microscopic movement of particles, a fiber-optic light was reflected on the field of view in the front and the back of the bed. The field of view in most experiments was a $2 \mathrm{~cm} \times 2 \mathrm{~cm}$ area. As the particles were fluidized inside the bed, the camera with a zoom lens $\mathbf{1 8 - 1 0 8} \mathbf{~ m m}$ 
and close up focus transferred its field of view to the monitor with streak lines. These streak lines represented the space traveled by the particles in a given time interval specified on the camera. The images were then captured and digitized by a microimaging board and analyzed using Image-Pro Plus software. Radial and axial velocity measurements were conducted at different locations inside the bed. The velocity vector was calculated as,

$$
\begin{aligned}
& v_{x}=\frac{\Delta L}{\Delta t} \cos \alpha \\
& v_{y}=\frac{\Delta L}{\Delta t} \sin \alpha
\end{aligned}
$$

where, $\ddot{A} L$ is the distance traveled, á is the angle from horizontal, $\ddot{A} t$ is the inverse of shutter speed, and $v_{x}$ and $v_{y}$ are the vertical and horizontal velocity components, respectively.

D. Viscosity Measurements using Brookfield Viscometer. The viscometer was placed at the top of the fluidized bed, and secured over the centerline of the bed. A cylindrical spindle (\#1 LV) of $0.9421 \mathrm{~cm}$ diameter, $7.493 \mathrm{~cm}$ effective length and overall height of $11.50 \mathrm{~cm}$ was used. The cylindrical spindle was attached to the bottom of the viscometer without the guard and was lowered inside the fluidized bed by an extension wire until it was completely immersed in the mixture during measurements.

The measurements in this experiment were made under LV mode at different speeds between of 2 and $20 \mathrm{rpm}$. At each rotational speed, between 10 and 30 readings were taken. The calibration of the viscometer-spindle apparatus was done using a Newtonian liquid, namely, water.

E. Granular Temperature Determination. The granular temperature, which is $3 / 2$ of the random particle kinetic energy, is obtained from the frequency distribution of the instantaneous velocities measured with the PIV system. Figure 4 shows typical distributions. The variances, ó $^{2}$, give the granular temperature, è, as shown below $\boldsymbol{\theta}=\frac{1}{3}\left[\sigma_{x}^{2}+\sigma_{y}^{2}+\sigma_{z}^{2}\right]$

Since no distributions were measured into the depth of the bed, the $\mathrm{z}$ direction, and since the variance in the direction of flow is the largest, in the calculation the assumption was made that the $\mathrm{z}$ direction variance equals the $\mathrm{x}$ direction variance. Hence

$\boldsymbol{\theta}=\frac{1}{3}\left[2 \sigma_{x}^{2}+\sigma_{y}^{2}\right]$

\section{Experimental Results for Bubbly Coalesced Regime.}

A. Phase Hold-Up. From the calibration curves of the X-ray and gamma-ray densitometers, the time average values of volume fraction for liquid, gas and solid phases were calculated. Tables 2 and 3 represent such a subset of the volume fraction of gas and 
solids at varying heights and two different horizontal positions. The particle and gas concentrations appear to be nearly constant throughout the region. A computer simulation of this system, using the experimental superficial liquid velocity of $2 \mathrm{~cm} / \mathrm{s}$ and gas velocity of $3.37 \mathrm{~cm} / \mathrm{s}$, also shows uniformity in solids concentration distribution. Figure 3 compares the volume fractions obtained from the computer simulation with the experimental results. Computer simulations will be discussed in detail in the next section.

B. Instantaneous Velocity Distribution. The measured velocity data were analyzed using frequency distribution plots. The frequency distribution plots for particles vertical and horizontal velocities are shown in Figures 4(a) and 4(b) for three phase fluidized bed.

C. Granular Temperature. Figures 5 shows a graph of the granular temperature, calculated using particle velocity measurements, as a function of horizontal distance from centerline of the bed at two different heights. The granular temperature of the 800 micron beads is about $200(\mathrm{~cm} / \mathrm{s})^{2}$, except near the left wall, where there is a higher velocity and more dilute flow due to the asymmetry in the system. This compares to about $1000(\mathrm{~cm} / \mathrm{s})^{2}$ for 500 micron beads in air, determined in the IIT CFB and about $10(\mathrm{~cm} / \mathrm{s})^{2}$ for 500 micron beads in water measured at IIT. Clearly a higher gas flow increased the turbulence of the system. For 45 micron methanol catalyst particles, Wu and Gidaspow(2000) computed the granular temperature to be between 20 and 10 for volume fractions corresponding to 0.1 nad 0.25 , respectively. These computations approximately agree with the measurements of Mostofi (2000). The lower value of the granular temperature is due to the smaller particle size.

D. Fluid Bed Viscosity. The viscosity of the glass beads in the mixture was obtained in two ways: 1 . from a direct measurement of the viscosity by a Brookfield viscometer and 2 . from the measurement of the random particle velocity using the equation

$$
\mu_{s}=\frac{5 \rho_{s} d_{s}(\pi \theta)^{\frac{1}{2}}}{48(1+e) g_{o}}\left[1+\frac{4}{5}(1+e) g_{o} \varepsilon_{s}\right]^{2}+\frac{4}{5}(1+e) g_{o} \varepsilon_{s}^{2} \rho_{s} d_{s}\left(\frac{\theta}{\pi}\right)^{\frac{1}{2}}
$$

Gidaspow and Huilin (1996) have shown that these two methods give the same value of the viscosity for 75 micron FCC particles in a riser. The same result holds here, since the large viscosity of the 800 micron beads exceeds the viscosity of water and air. Figure 6 shows that the viscosities are the same within experimental error.

\section{Part II. SIMULATION}

Hydrodynamic Model

A transient, isothermal, three-dimensional model for multiphase flow was developed. The hydrodynamic model uses the principle of mass conservation and momentum balance for each phase. This approach is similar to that of Soo(1967) for multiphase flow and of Jackson (1985) for fluidization. The equations are similar to Bowen's (1976) balance laws for multi-component mixtures. The principle difference is the appearance of the volume fraction of phase " $k$ " denoted by $\stackrel{a}{k}_{k}$. The fluid pressure, $\mathrm{P}$, is in the liquid (continuous) phase.

For gas-solid fluidized beds, Bouillard, et al. (1989) have shown that this set of equations produces essentially the same numerical answers for fluidization as did the earlier 
conditionally stable model, which has the fluid pressure in both the gas and the solids phases. In this model (hydrodynamic model B), the drag and the stress relations were altered to satisfy Archimedes' buoyancy principle and Darcy's Law, as illustrated by Jayaswal (1991). Note in Table 4, no volume fraction is put into the liquid gravity term, while in the gas/solid momentum balance contains the buoyancy term. This is a generalization of model B for gas-solid systems as discussed by Gidaspow(1994) in section 2.4. This model is unconditionally well-posed, ie, the characteristics are real and distinct for one-dimensional transient flow. It does not require the presence of solid's pressure for stability and well-posedness.

The numerical method is an extension of Harlow and Amsden's(1971) method, which was subsequently used in the K-FIX program (Rivard and Torrey, 1977). The present program was developed from Jayaswal's two-dimensional MICE program (1991); which originated from the K-FIX program (Rivard and Torrey, 1977). To obtain the numerical solution, the non-uniform computational mesh is used in finite-differencing the equations based on the ICE, implicit Eularian method (Rivard, 1977; Jayaswal, 1991) with appropriate initial and boundary conditions. Stewart and Wedroff (1984) have critically reviewed the ICE algorithm and related staggered mesh conservative schemes. The scalar variables are located at the cell center and the vector variables at the cell boundaries. The momentum equation is solved using a staggered mesh, while the continuity equation is solved using a donor cell method.

Table 4 shows the continuity and the separate phase momentum equations for threedimensional transient three-phase flow. There are nine nonlinear-coupled partial differential equations for nine dependent variables. The variables to be computed are the volume fractions, $\stackrel{\circ}{\text { phases- }}_{1}$, the liquid phase pressure $\mathrm{P}$, and the phase horizontal, $\mathrm{x}$ direction, and vertical velocity, $y$-direction components, $u_{\text {phase }}$ and $v_{\text {phase. The gradient of }}$ pressure is in the fluid (continuous) phase only. This leads to an unconditionally wellposed problem, as discussed in detail by Gidaspow(1994) and Lyczkowski, et al. (1978). A value of 10 poises times the particle concentration was used throughout the simulations. This equation was obtained by fitting the experimental viscosity values at given superficial liquid and gas velocities (Bahary, 1994). Therefore, the viscous stress terms for the phases are of the Newtonian form as follows

$$
\begin{gathered}
\left.\overline{\overline{\tau_{k}}}\right]=2 \varepsilon_{k} \mu_{k}\left[\overline{\overline{S_{k}}}\right] ; \quad \mu_{k}=\varepsilon_{k} 10(\text { poises }) \\
{\left[\overline{\overline{S_{k}}}\right]=\frac{1}{2}\left[\nabla \vec{v}_{k}+\left(\nabla \vec{v}_{k}\right)^{T}\right]-\frac{1}{3}\left[\nabla \cdot \overrightarrow{\mathrm{v}}_{\mathrm{k}} \overline{\bar{I}}\right]}
\end{gathered}
$$

The solids' pressure is calculated by the solids stress modulus using the following equations, $\nabla P_{k}=G\left(\varepsilon_{k}\right) \nabla \varepsilon_{k} ; \quad G\left(\varepsilon_{k}\right)=10^{-8.686 \varepsilon_{k}+6.385}$

As a particle moves through a viscous liquid there exists a resistance of the liquid to the motion of the particle, hence the interphase drag has to be defined. One type is the packedpressure drop data expressed in the form of a correlation, such as the Ergun equation.

For $\stackrel{\circ}{k}_{k} 0.2$ 


$$
\boldsymbol{\beta}_{f k}=\beta_{k f}=150 \frac{\left(1-\varepsilon_{f}\right) \varepsilon_{k} \mu_{f}}{\left(\varepsilon_{f} d_{k} \boldsymbol{\Psi}_{k}\right)^{2}}+1.75 \frac{\varepsilon_{k} \rho_{f}\left|\vec{v}_{f}-\vec{v}_{k}\right|}{\varepsilon_{f} d_{k} \boldsymbol{\Psi}_{k}}
$$

For $\stackrel{\circ}{k}_{k}<0.2$, the empirical correlation is based on Stoke's drag coefficient $\mathrm{C}_{\mathrm{d}}$ as follows

$$
\begin{aligned}
& \beta_{f k}=\beta_{k f}=3 / 4 C_{D} \frac{\varepsilon_{k} \varepsilon_{f}^{-2.65} \rho_{f}}{d_{k} \Psi_{k}}\left|\vec{v}_{f}-\vec{v}_{k}\right| \\
& \text { where } \begin{aligned}
& C_{D}=\frac{24}{R e_{k}}\left[1+0.15 \mathrm{Re}_{k}^{0.687}\right], \text { for } \operatorname{Re}_{k}<1000 \\
& C_{D}=0.44, \text { for } \operatorname{Re}_{k} \geq 1000
\end{aligned}
\end{aligned}
$$

Arastoopour, Lin and Gidaspow (1980) observed that solid-solid momentum transfer is necessary to correctly predict the segregation among particles of different sizes in a pneumatic conveyor. Particle-particle drag equations to describe such interactions have been derived by several researchers: Soo (1967), Nakamura and Capes(1976) and Syamlal (1985). In the present work the drag coefficient is based on kinetic theory (Syamlal, 1985) represented as

$$
\beta_{k l}=\frac{\alpha(1+e) \varepsilon_{k} \rho_{k} \varepsilon_{l} \rho_{l}\left(d_{k}+d_{l}\right)^{2}\left[1+3\left(\frac{\varsigma_{k l}}{\varepsilon_{k}+\varepsilon_{l}}\right)^{\frac{1}{3}}\right]}{2 \varepsilon_{f}\left(\rho_{k} d_{k}^{3}+\rho_{l} d_{l}^{3}\right)\left[3\left(\frac{\varsigma_{k l}}{\varepsilon_{k}+\varepsilon_{l}}\right)^{\frac{1}{3}}-1\right]}\left|\vec{v}_{k}\right|
$$

where

$$
\begin{aligned}
& \int\left[\left(\phi_{k}-\phi_{l}\right)+(1-\alpha)\left(1-\phi_{k}\right) \phi_{l}\right]\left[\phi_{k}+\left(1-\phi_{l}\right) \phi_{k}\right] \frac{\chi_{k}}{\phi_{k}}+\phi_{l} \\
& \text { for } \chi_{k} \leq \frac{\phi_{k}}{\left[\phi_{k}+\left(1-\phi_{k}\right) \phi_{l}\right]} \\
& (1-\alpha)\left[\phi_{k}+\left(1-\phi_{k}\right) \phi_{l}\right]\left(1-\chi_{k}\right)+\phi_{k} \\
& \text { for } \chi_{k}>\frac{\phi_{k}}{\left[\phi_{k}+\left(1-\phi_{k}\right) \phi_{l}\right]} \\
& \alpha=\sqrt{\frac{d_{l}}{d_{k}}} \quad d_{k} \geq d_{i} \\
& \chi_{k}=\frac{\varepsilon_{k}}{\varepsilon_{k}+\varepsilon_{f}}
\end{aligned}
$$

The gas phase is treated as a particulate phase by specifying the injected air bubble diameter, viscosity and density as visually seen in experiment. The simulation results are time-average as follows

$$
<v(x, y, z)>=\frac{1}{t_{o}} \int_{t}^{t+t_{o}} v(x, y, z, t) d t(16)
$$


The other corresponding equations used to analyze the output from the simulation are summarized in Table 5. These equations are a result from time-averaging the equationsof continuity and of motion (Bird et al., 1960). These terms are the components of the turbulent momentum flux and are referred to as the Reynolds stresses.

Coordinate system and numerical considerations

The solution of the preceding conservation equations depends on the definition of boundary conditions for adequate comparison to experiment. The diameter of the leaded glass beads was $0.889 \mathrm{~cm}$ with a density of $2.49 \mathrm{~g} / \mathrm{cm}^{3}$. The viscosity was an input in all simulations to match experimentally obtained viscosities as discussed previously.

Different inlet conditions and grid sizes were prescribed to test the sensitivity of the final flow field solution and are summarized as cases in Table 6. Figure 7illustrates the twodimensional computational domain for case FB2d3d in Table 6. The third dimension, to represent the experimental $5.08 \mathrm{~cm}$ depth of the bed, is added with a grid size of $1.02 \mathrm{~cm}$. It will be shown that the two-dimensional simulation can properly represent the flow hydrodynamics and be less computer time intensive. The remainder of this section will be to study the effects of varying grid size, injected air bubble diameter and void fraction as represented in Table 4. The left side is taken to be the inlet from left wall to centerline of the horizontal, $15 \mathrm{~cm}$.

Three Dimensional Simulation: Case FB2d3d in Table 6

Flow Field and Averaged Velocity Profiles

Figures $8 \mathrm{a}$ and $8 \mathrm{~b}$ show the three-dimensional time-averaged, 16 to 42 seconds, gas and solid volume fraction contour plots along with the time-averaged velocity vectors at the $x-y$ plane of $3 \mathrm{~cm}$ from the front wall. Figure $8 \mathrm{c}$ shows the solid contour plot with corresponding velocity vectors at a time of 39 seconds in the $y-z$ plane of $17.5 \mathrm{~cm}$ from the left wall. The computed flow pattern correctly shows gas up flow in the center region as visually confirmed in the experiment. A video of the experiment and of the simulations shows that the solid fluctuates upward and downward in the center region. Time-averaged velocities show the solid moving downward in the center as illustrated in Figure $8 \mathrm{~b}$. The phenomenon of downward flow of particles in the center is almost identical to that observed experimentally in a two dimensional gas-solid fluidized bed at low gas velocities, where the bubbles form between the center of the bed and the walls, as shown in Fig 10.5 b in Gidaspow's ( 1994) book. His figure 10.5 a shows the corresponding computations. His vorticity equation 10.46 explains the phenomenon quantitatively. Once bubbles form near the walls, producing upward motion of particles in this region, conservation of mass of particles forces them to descend in the center. Figure 9 reveals that the two-dimensional time-averaged vertical velocities overlap the three-dimensional time-averaged velocity pattern and both agree well with experiment. Figure 10 further illustrates that the solids vertical velocity does not change when going into the bed. Figure 11 further demonstrates the time-averaged water downward vertical velocities in the center region. This down flow in the center produces a rotation or particle vorticity as seen in Figure 8a. Due to the buoyancy air moves up only. Reynolds Stresses

The stresses are calculated from the velocity vectors directly using equations presented in Table 5. The profiles of Figures 12 and 14 and all cases studied show that the Reynolds 
stress <u'u'> peaks in the center, whereas 〈v'v'> peaks close to the walls in agreement with Muddle, et al., (1997) and Pan, et al., (2000) for gas-liquid flow only. The explanation of the appearance and diagonal vortical movement by Muddle, et al., (1997) causes drastic swings in the vertical velocity close to the wall, where the motion is primarily upward. In the center, the horizontal velocity attains its highest magnitude, contributing the most to the horizontal stresses in the center, but the least by the wall. At the left wall, the vertical Reynolds stress is the highest, thus the granular temperature exhibits this maximum at the wall as shown in Figure 13. From experiment, Figure 5, the same characteristic experimental maximum turbulence is observed closer to the left wall; even at the lower inlet water and air superficial velocities. Figure 14 shows the stresses plotted into the depth at z-y plane of $12 \mathrm{~cm}$ from the left wall. The horizontal stress exhibits the characteristic maximum in the center region, but the vertical Reynolds stress is much flatter. This can be attributed to no vortex formation in the third direction. Two-Dimensional Low Velocity Simulations

Flow Field and Averaged Velocity Profiles

Figure 15 represents the time-averaged, 15 to 44 seconds, solids contour plot and velocity vectors for Case FB5. Figure 16a illustrates the time-averaged solids' contour plot and velocity vectors for Case FB2. The only difference between these two cases is the injected air bubble diameter and grid size. From Figures 18 through 20 it will be shown that grid size did not affect the transient behavior of the bed. Figure 15 and 16a show the difference in the bed expansion is due to the increase in bubble diameter. Figures 16b-c illustrate the instantaneous vortex movement from 14 seconds to 15 seconds for Case FB2.

Figure 17 shows the power spectrum of the vertical velocity for Case FB2 at $8 \mathrm{~cm}$ from left wall and at a bed height of $11 \mathrm{~cm}$. Muddle, et al., (1997) had found a similar low frequency peak and no dominant frequency above $1 \mathrm{~Hz}$ and Bahary (1995) has measured similar low frequencies. The time-averaged vertical and horizontal velocities are presented in Figure 18 and agree with the experimental averages, Figure 4, of $2.3 \mathrm{~cm} / \mathrm{s}$ for the vertical velocity and $-4.32 \mathrm{~cm} / \mathrm{s}$ for the horizontal velocity at a Bed Height of $9 \mathrm{~cm}$ and Horizontal Position of $7.5 \mathrm{~cm}$ from Left Wall. Figures 18, 19, and 20 represent the time-averaged velocities for the remaining cases with an injected air bubble diameter of $0.01 \mathrm{~cm}$. The agreement of the time-averaged velocities of Figures 18, 19 and 20 is expected, since the grid size is the only thing that varies between these cases. However, a noticeable difference exists in these Figures and the two proceeding Figures, 21 and 22. Figure 21 represents the time-averaged horizontal and vertical particle velocity profiles for a uniform inlet with only an inlet bubble diameter increase. These profiles are much flatter due to the lack of vortical structure production. Figure 22 represents the larger injected air bubble diameter and not symmetric inlet conditions (Case FB5). This velocity plot is not flat; instead, there exists an increase in the velocity in the half of the bed where more gas is injected.

Reynolds Stresses and Granular Temperature Figures 23, 24 and 25 show how damping of the stresses occurs by an increase in grid size. From Figure 23 to 24 , the y-directional grid cell is halved in size from $2.5 / 4.5 \mathrm{~cm}$ to $1 \mathrm{~cm}$. The shape of the curves remains the same, but the stresses are doubled. The same pattern can be seen when going from Figure 25 to 23, where the x-directional grid cell is halved in length and the stresses are double in size. Thus, the stresses appear to be 
linearly proportional to the grid size and is due to the small calculated stress values for this three-phase system. However, this grid size dependency is not seen with the granular temperature.

The granular temperature is compared to experiment in Figure 26 and shows general agreement. The damping in the stresses can also be seen in the granular temperature as the mean fluctuation of the velocity decreases so does the granular temperature. Figure 27 represents the granular temperature of the larger inlet bubble diameter (Case FB4) and as the time-averaged velocity profile was flattened, so is the granular temperature. The test for developed flow, as in DNS for single-phase, was performed on the cases. Figure 28 presents the test for developed flow of case FB3. The following equations are used to obtain the curves

$$
\begin{aligned}
& \int-\frac{d P}{d y}-g \sum_{i=1}^{3}\left(\varepsilon_{i} \rho_{i}\right) d x=\text { pressuredropminusweightof bed } \\
& \left\langle u^{\prime} v^{\prime}\right\rangle \sum_{i=1}^{3}\left(\varepsilon_{i} \rho_{i}\right)=\text { principal ReynoldsStress }
\end{aligned}
$$

The two expressions are not equal because the $\mathrm{u}$ and $\mathrm{v}$ velocities, see Figure 19, are of the same order of magnitude due to the vortex formation. The stresses shown in Figure 28 are on the order of magnitude of the solid's pressure, which is approximately equal to $P_{s}=\varepsilon_{s} \rho_{s} v_{s}^{2}$

This value is small as compared to the fluid pressure.

Discussion

Computations of the granular temperature and frequency allow us to speculate concerning vortex size from dimensional analysis and approximate solutions of NavierStokes equations for standing waves(Tolstoy,1973). Characteristic length equals the pseudosonic velocity divided by the major frequency; where the pseudosonic velocity equals the square root of the granular temperature. The granular temperature ranges between 50 and 100 from Figure 26 and major frequency is taken to be $0.3 \mathrm{~Hz}$. Entering these ranges into the equation gives us vortex sizes of 20 and $30 \mathrm{~cm}$. Hence, in our system we expect to have one to two vortices and do not expect any vortices into the depth. Figure 8 shows that there is no vortex in the third dimension. Figure $16 \mathrm{~b}-\mathrm{d}$ better illustrates the instantaneous single and double vortices generated by the code and also visible seen in experiment. This fluctuating particle vorticity and the flow of the liquid cause the particle concentration to be uniform throughout the bed. That is in contrast to the case of no liquid flow, where there is a vertical density gradient (Wu and Gidaspow, 2000). Such a catalyst distribution is reasonable. It is usually modeled using a sedimentation model (Viking, 1993). In production of gasoline in a FCC riser, there exists a sharp radial catalyst gradient. In view of this undesirable distribution of particles, other designs such as the downer are being considered. Hence, the discovery of a uniform concentration described in this study is of some practical significance. In the future, this model will be explored further. Further, its' principle weakness is in the uncertainty of the bubble size. Figure 15 and 16 show an order of magnitude difference in bed expansion caused by the order of magnitude increase in bubble diameter. Such an effect is reasonable, since the bed expands a lot more for fine particles or fine bubbles. 
Conclusions

1. A transient, three-dimensional computer code for the solutions of the coupled Navier-Stokes equations for gas-liquid-solid flow was developed.

The principal input is the particulate viscosity, which was measured with a Brookfield viscometer and a PIV technique.

2. The computed time-averaged particle velocities and concentrations agree with measurements done in the slurry bubble column with continuous flow of liquid in the churn-turbulent regime. The particle velocities were measured using the PIV technique. The concentrations were determined using a combination of gamma-ray and x-ray densitometers. Both the experiment and the simulations show a downflow of particles in the center of the column with upflow near the wall. The situation is unlike the case of no liquid recirculation (Wu and Gidaspow, 2000) where there exist large inhomogeneities of particles in the bed.

3. Computed instantaneous particle velocities were used to construct normal and shear Reynolds stresses, similar to the procedure in DNS for singlephase flow. The computed horizontal distributions of granular temperature, the turbulent kinetic energy of particles, agreed with measurements done using a PIV technique.

Acknowledgements

This study was partially supported by Department of Energy Grant No. DE-FG-

98FT40117.

$\begin{array}{ll}\text { Nomenclature } & \\ \text { Abbreviation } & \text { Term } \\ \mathrm{C}_{\mathrm{D}} & \text { drag coefficient } \\ \mathrm{d}_{\mathrm{k}} & \text { characteristic particulate phase diameter } \\ \mathrm{E} & \text { coefficient of restitution } \\ \mathrm{G} & \text { gravity } \\ \mathrm{G} & \text { solid compressive stress modulus } \\ \mathrm{g}_{\mathrm{o}} & \text { radial distribution function at contact } \\ \mathrm{P} & \text { Continuous phase pressure } \\ \mathrm{P}_{\mathrm{k}} & \text { Dispersed(particulate) phase pressure } \\ \mathrm{Re}_{\mathrm{k}} & \text { Reynolds number for phase } \mathrm{k} \\ \mathrm{T} & \text { time } \\ \mathrm{U} & \text { horizontal velocity, } \mathrm{x} \text {-direction } \\ \mathrm{V} & \text { vertical velocity, } \mathrm{y} \text {-direction } \\ \mathrm{W} & \text { depth velocity, } \mathrm{z} \text {-direction } \\ & \\ \mathrm{Greek} \text { Letters } & \\ \hat{\mathrm{a}}_{\mathrm{km}} & \text { interphase momentum transfer coefficient } \\ & \text { between } \mathrm{k} \text { and } \mathrm{m} \\ \mathrm{a}_{\mathrm{k}} & \text { volume fraction of phase } \mathrm{k} \\ \mathrm{E} & \text { granular temperature }\end{array}$




$\begin{array}{ll}\grave{\mathrm{I}} & \text { viscosity } \\ \tilde{\mathrm{N}} & \text { density } \\ \hat{\mathrm{O}}_{\mathrm{k}} & \text { stress } \\ \phi_{k} & \text { solids' volume fraction at maximum packing } \\ \varnothing & \text { particle sphericity }\end{array}$

\section{Literature Cited}

Anderson, K., S. Sundaresan and R. Jackson, "Instabilities and the Formation of Bubbles in Fluidized Beds," J. Fluid Mech., 303, 327-366(1995).

Ahmadi, G. and D. Ma, "A Thermodynamical Formulation for Dispersed Multiphase Turbulent Flows-I,” Int. J. of Mutiphase Flow, 16, 323-340(1990).

Arastoopour, H., D. Lin and D. Gidaspow, "Hydrodynamic Analysis of Pneumatic Transport of a Mixture of Two Particle Sizes," in "Multiphase Transport," ed. T.N. Veziroglu, Hemisphere Pub. Corp., 4, 1853-1871(1980).

Bahary, M., "Experimental and Computational Studies of Hydrodynamics in Three-Phase and Two-Phase Fluidized Beds," PhD Thesis, Illinois Institute of Technology, Chicago (1994).

Bechtel Group, "Slurry Reactor Design Studies. Slurry vs. Fixed Bed Reactors For Fischer-Tropsch and Methanol: Final Report, No. DE91005752, Jun. 1990.

Benyahia S. , H. Arastoopour, and T. Knowlton, "Prediction of Solid and Gas Flow Behavior in a Riser Using a Computational Multiphase Flow Approach," Fluidization X, Proceedings of the Ninth Eng. Found. Conf. On Fluidization, edited by L.-S. Fan and T. Knowlton, New York, (1998).

Bird, R.B., W.E. Stewart and E.N. Lightfoot, "Transport Phenomena," John Wiley and Sons, (1960).

Bouillard, J.X. and D. Gidaspow, "Porosity Distribution in a Fluidized Bed with an Immersed Obstacle," A.I.Ch.E. Journal, 35, 908-922(1989).

Bowen, R.M., "Theory of Mixtures," Continuum Pysics, Vol. III, editor A.C. Eringen, Academic Press, New York (1976).

Courant, R., E. Isaacson and M. Rees, "On the Solution of Nonlinear Hyperbolic Differential Equations by Finite Differences," Comm. in Pure and Applied Mathematics, 5, 243(1952).

Crowe, C., M. Sommerfeld, and Y. Tsuji, Multiphase Flows with Droplets and Particles, CRC Press, Boston, (1998).

Fan, L.-S., "Gas-Liquid-Solid Fluidization Engineering,” Butterworths, Boston, Mass. (1989).

Gidaspow, D., "Multiphase Flow and Fluidization: Continuum and Kinetic Theory Descriptions," Academic Press, New York, NY., (1994).

Gidaspow, D. M. Bahary and Y. Wu, "Hydrodynamic Models for Slurry Bubble Column Reactors," Proceedings of Coal Liquefaction and Gas Conversion Contractors Review Conference, PP. 397-401, DOE/PETC, Aug. 1995. 
Gidaspow, D., and L. Huilin, "Collisional Viscosity of FCC Particles in a CFB,"

A.I.Ch.E. Journal, 42, 2503-2510(1996).

Gidaspow, D., and L. Huilin, "Equation of State and Radial Distribution Functions of FCC Particles in a CFB," A.I.Ch.E. Journal, 44, 279-293(1998).

Grevskott, S. B.h. Sannaes, M.P. Dudukovic, K.W. Hjarbo and H.F. Svendsen, "Liquid Circulation, Bubble Size Distribtions and Solid Movement in Two- and ThreePhase Bubble Columns," Chem. Eng. Sci., 51, 1703-1713(1996).

Harlow, F.H and A. Amsden, "A Numerical Fluid Dynamics Calculation Method for all Flow Speeds," Jour. of Comp. Physics, 8, 197-213(1971).

Heard, W.B. and G.R. Richter, "Numerical Mutiphase, Multicomponent Flow Modeling," Computational Fluid Dynamics in Chem. Reaction Eng., United Eng. Found., San Diego, Ca., (Oct. 13-18,1996).

Jackson, R., "Hydrodynamic Stability of Fluid-Particle Systems," in Fluidization, edited by J.F.Davidson, R. Cliff and D. Harrison, Academic Press, New York, 4772(1985).

Jayaswal, U.K., "Hydrodynamics of Multiphase Flows: Seperation, Dissemination and Fluidization," Ph.D. Thesis, Illinois Institute of Technology, Chicago (1991).

Kashiwa, B.A. and W. B. VanderHeyden, "An Extended K-Epsilon Turbulence Model for Multiphase Flow," Thirteenth U.S. National Congress of Applied Mechanics, Univerisity of Florida, Gainesville, Fl., (June 21-26, 1998).

Kwaguchi, K., T. Tanaka and Y. Tsuji, "Numerical Simulation of Two-dimensional Fluidized Beds using the Discrete Element Method (Comparison between the Two- and Three-Dimensional Models)," Powder Tech., 96, 129-138(1998).

Kim, John, P. Moin and R. Moser, "Turbulence Statistics in Fully Developed Channel Flow at Low Reyolds Number," J. Fluid Mec. 177, 133-166(1987)

Krishna, R. M.I. Urseanu, J.M. Van Baten and J. Ellenberger, "Influence of Scale on the Hydrodynamics of Buble Columns Operating in the Churn-Turbulent Regime: Experiments vs. Eulerian Simulations," Chem. Eng. Sci., 54, 4903-4911(1999).

Li, Y. J. Zhang and Liang-Shih Fan, "Numerical Simulation of Gas-Liquid-Solid Fluidization Systems using a Combined CFD-VOF-DPM Method: Bubble Wake Behavior,” Chem. Eng. Sci., 54, 5101-5107(1999).

Lyczkowski, R.W., D. Gidaspow, C.w.Solbrig and E.C. Hughes, "Characteristics and Stability Analysis of Transient One-dimensional Two-Phase Flow Equations and their Finite Difference Aproximations," Nuclear Science and Eng., 66, 378396(1978).

Mathiesen, V., T. Solberg, H. Arastoopour and B. Hjertager, "Experimental and Computational Study of Multiphase Gas/Particle Flow in a CFB Riser," A.I.Ch.E. Journal, 45, 2503-2518 (1998).

Matonis, D., "Hydrodynamic Simulation of Gas-Liquid-Solid Fluidized Bed," PhD Thesis, Illinois Institute of Technology, Chicago (2000).

Miller, A. and D. Gidaspow, "Dense, Vertical Gas-Solid Flow in a Pipe," A.I.Ch.E. Journal, 38, 1801-1815 (1992).

Mostafi, R., PhD Thesis in progress, (2000).

Mitra-Majumdar, D., B. Farouk and Y.T. Shah, "Hydrodynamic Modeling of Threephase Flows through a Vertical Colum,” Chem. Eng. Sci., 52, 4485-4497(1997). 
Muddle, R.F., D.J. Lee, J. Resse and L.-S. Fan, "Role of Coherent Structures on Reynolds Stresses in a 2-D Bubble Column," A.I.Ch.E. Journal, 43, 913926(1997).

Nakamura, K. and C.E. Capes, "Vertical Pneumatic Conveying of Binary Particle Mixtures," Fluidization Technology, Vol. II, New York, 1976.

Neri, A., D. Gidaspow, "Riser Hydrodynamics: Simulation Using Kinetic Theory,"

A.I.Ch.E. Journal, 46, 52-67(2000).

Nikitin, N.V, "A Study of the Near-Wall Turbulent Flows via Direct Numerical Simulations," XIXth International Congress of Theoretical and Applied Mechanics, Kyoto Japan, 451(August 25-31,1996).

NICHE, The Council for Chemical Research New Industrial Chemistry and Eng., "Computational Chemistry and Fluid Dynamics," Marco Island, Florida, June 11$14,2000$.

Pan, Y., M.P. Dudukovic, M. Chang, "Numerical Investigation of Gas-Driven Flow in 2D Bubbble Columns," AIChE J., 46, 434-448(2000).

Parkinson, G., "Fischer-Tropsh Comes Back," Chem. Eng., 104, 39-41(1997).

Pfleger, D., S. Gomes, N. Gilbert, h.-G. Wagner, "Hydrodynamic Simulations of Laboratory Scale Bubble Columns Fundamental Studies of Eulerian-Eulerian Modeling Approach," Chem. Eng. Sci., 54, 5091-5099(1999).

Prosperetti,A., "Numerical Aspects of the Simmer-11 Code," Multiphase Process in LMFBR Safety Analysis, Ispra, Italy, April, 974-224 (1982).

Rivard, W.C., and M.D. Torrey, "K-FIX: A Computer Program for Transient Twodimensional, Two-Fluid Flow," LA-NUREG-6623, Los Alamos (1977).

Samuelsberg, A., and B.H. Hjertager, "Computational Moeling of Gas/Particle in a Riser," A.I.Ch.E. Journal, 42, 1536-1546(1996).

Seo, Y. and D. Gidaspow, "An X-ray-gamma-ray of Measurement of Binary Solids Concentrarions and Void in Fluidized Beds," Ind. Eng. Chem. Res., 26, 16221628 (1987).

Soo, S.L., "Fluid Dynamics of Multiphase Systems," Blaisdell Publ. Co., Waltham, Ma., (1967).

Sinclair, J.L. and R. Jackson, "Gas-Particle Flow in a Vertical Pipe with Particle-Particle Interactions," AIChE J., 35, 1473(1989).

Stewart, H.B. and B. Wendroff, “Two-Phase Flow: Models and Methods," J. Comput. Phys., 56, 363-409 (1984).

Symlal, M. "Multiphase Hydrodynamics of Gas-Solid Flow," Ph.D. Thesis, Illinois Institute of Technology, Chicago, Il., (1985).

Tarmy, B.L. and C.A. Coulaloglou, "Alpha-Omega and Beyond Industrial View of Gas/Liquid/Solid Reactor Development," Chem. Eng. Sci., 47, 3231-3246(1992).

Thompson, T, "The Mutiphase Fluid Dynamics Research Consortium." See "Vision 2020 Comes into Focus," C\&EN News, Aug.9, 1999.

Tolstoy, I., "Wave Propagation," New York, McGraw-Hill (1973).

Tsuo, Y.P. and D. Gidaspow, "Computations of Flow Patterns in Circulating Fluidized Beds," A.I.Ch.E. Journal, 36, 885-896 (1990).

Tsuji, Y, T. Tanaka and S. Yonemura, "Cluster Patterns in Circulating Fluidized Beds Predicted by Numerical Simulation (Discrete Particle Model versus Two-Fluid Model)," Powder Technology, 95, 254-264(1998). 
Vikings System International, "Design of Slurry Reactor for Indirect Liquefaction Applications," Report to DOE/PETC by A. Prakash and P.B. Bendale, DE-AC2289PC89870.

Wu, Y. and D. Gidaspow, "Hydrodynamic Simulation of Methanol Synthesis in GasLiquid Slurry Bubble Column Reactors," Chem. Eng. Sci., 55, 573-587(2000).

Xu, B.H., A.B. Yu, "Numerical Simulation of the GAS-Solid Flow in a Fluidized Bed by Combinin Discrete Particle Method with Computational Fluid Dynamics," Chem. Eng. Sci., 52,2785- 2809(1997). 


\begin{tabular}{ll}
\hline TABLE 1. Operating Conditions for Bubbly Coalesced Regime Experiments \\
\hline & \\
Temperature $\left({ }^{\circ} \mathrm{C}\right)$ & 23.5 \\
Particle Mean Diameter $(\mathrm{cm})$ & 0.8 \\
Particle Density $\left(\mathrm{g} / \mathrm{cm}^{3}\right)$ & 2.94 \\
Initial Bed Height $(\mathrm{cm})$ & $22 / 24$ \\
Minimum Fluidization Velocity $(\mathrm{cm} / \mathrm{s})$ & 0.76 \\
\hline
\end{tabular}

Table 2. Measured Phase Hold-up in Bubble Coalesced Regime for $V_{\text {gas }}=3.37 \mathrm{~cm} / \mathrm{s}$ at $11 \mathrm{~cm}$ from the Left Wall.

\begin{tabular}{l|lllllll}
\hline Bed heights, cm & 2.5 & 5 & 7.5 & 10 & 12.5 & 15 & 17.5 \\
$\mathfrak{a}_{\text {solid }}$ & 0.25 & 0.18 & 0.15 & 0.16 & 0.2 & 0.25 & 0.2 \\
$\mathfrak{a}_{\text {air }}$ & 0.56 & 0.4 & 0.34 & 0.36 & 0.36 & 0.3 & 0.32
\end{tabular}

Table 3. Measured Phase Hold-up in Bubble Coalesced Regime for $\mathrm{V}_{\text {gas }}=3.37 \mathrm{~cm} / \mathrm{s}$ at $-2 \mathrm{~cm} \mathrm{~cm}$ from the Left Wall.

\begin{tabular}{l|lllllll}
\hline Bed heights, cm & 2.5 & 5 & 7.5 & 10 & 12.5 & 15 & 17.5 \\
$\stackrel{a}{\text { asolid }}_{\mathbf{a}_{\text {air }}}$ & 0.15 & 0.21 & 0.08 & 0.12 & 0.1 & 0.15 & 0.16 \\
& 0.5 & 0.43 & 0.4 & 0.41 & 0.35 & 0.41 & 0.53
\end{tabular}


Table 4. Governing Equations

Continuity Equation for Each Phase, $k=g, l, s$

$\frac{\partial}{\partial t}\left(\varepsilon_{k} \rho_{k}\right)+\nabla \cdot\left(\varepsilon_{k} \rho_{k} \vec{v}_{k}\right)=0$

Continuous Phase (Liquid) Momentum Balance

$\frac{\partial}{\partial t}\left(\varepsilon_{f} \rho_{f} \vec{v}_{f}\right)+\nabla \cdot\left(\varepsilon_{f} \rho_{f} \vec{v}_{f} \vec{v}_{f}\right)=-\nabla \cdot P \overline{\bar{I}}+\rho_{f} \vec{g}+$

$$
\sum_{m=g, s} \beta_{f m}\left|v_{m}-v_{f}\right|+\nabla \cdot \tau_{f}^{=}
$$

Dispersed Phase (Gas or Solid) Momentum Balance

$$
\begin{gathered}
\frac{\partial}{\partial t}\left(\varepsilon_{k} \rho_{k} \vec{v}_{k}\right)+\nabla \cdot\left(\varepsilon_{k} \rho_{k} \vec{v}_{k} \vec{v}_{k}\right)=-\nabla P_{k} \bar{I}+\frac{\varepsilon_{k}}{\varepsilon_{f}}\left(\rho_{k}-\sum_{m=f g, s} \varepsilon_{m} \rho_{m}\right) \vec{g}+ \\
\sum_{\substack{m=, g, s \\
m \neq k}} \beta_{f m}\left|\vec{v}_{m}-\vec{v}_{k}\right|+\nabla \cdot \tau_{k}
\end{gathered}
$$

Viscous Stress Tensor

$$
\begin{gathered}
{\left[\bar{\tau}_{f}\right]=2 \varepsilon_{k} \mu_{k}\left[\overline{\overline{S_{f}}}\right]} \\
{\left[\overline{\overline{S_{f}}}\right]=\frac{1}{2}\left[\nabla \vec{v}_{f}+\left(\nabla \vec{v}_{f}\right)^{T}\right]-\frac{1}{3}\left[\nabla \cdot \overrightarrow{\mathrm{v}}_{\mathrm{f}} \overline{\bar{I}}\right]}
\end{gathered}
$$


Table 5. Equations of the calculated stresses.

$\left\langle v^{\prime} v^{\prime}\right\rangle=\left[\frac{1}{N(t)} \sum v(x, y, z, t) v(x, y, z, t)\right]-(<v(x, y, z)>)^{2}$

$<u^{\prime} v^{\prime}>=<v^{\prime} u^{\prime}>=\frac{1}{N(t)} \sum[\{u(x, y, z, t)-<u(x, y, z)>\}\{v(x, y, z, t)-<v(x, y, z)>\}]$

$<u^{\prime} u^{\prime}>=\left[\frac{1}{N(i)} \sum u(x, y, z, t) u(x, y, z, t)\right]-(<u(x, y, z)>)^{2}$

$<u^{\prime} w^{\prime}>=<w^{\prime} u^{\prime}>=\frac{1}{N(t)} \sum[\{u(x, y, z, t)-<u(x, y, z)>\}\{w(x, y, z, t)-<w(x, y, z)>\}]$

$<w^{\prime} w^{\prime}>=\left[\frac{1}{N(i)} \sum w(x, y, z, t) w(x, y, z, t)\right]-(<w(x, y, z)>)^{2}$

$<v^{\prime} w^{\prime}>=<w^{\prime} v^{\prime}>=\frac{1}{N(t)} \sum[\{v(x, y, z)-<v(x, y, z)>\}\{w(x, y, z)-<w(x, y, z)>\}]$

with $N(t)$ being the number of vectors in the time-average

Table 6. Simulation Cases under Investigation

\begin{tabular}{|c|c|c|c|c|c|c|c|c|}
\hline Case & $\ddot{\mathrm{A} x}, \mathrm{~cm}$ & Äy, cm & $\begin{array}{l}\mathrm{V}_{\text {Liquid }} \\
\mathrm{cm} / \mathrm{s}\end{array}$ & $\begin{array}{l}\mathrm{V}_{\mathrm{Gas}} \\
\mathrm{cm} / \mathrm{s}\end{array}$ & $\begin{array}{l}\mathrm{D}_{\text {air }}, \\
\mathrm{cm}\end{array}$ & $\begin{array}{l}\stackrel{\circ}{\text { left,wate }}_{\text {r }} \\
\text { lat }\end{array}$ & $\mathrm{å}_{\text {left,gas }}$ & $\begin{array}{l}\stackrel{\circ}{\text { aright,wat }}= \\
\text { er }= \\
\stackrel{\circ}{\text { right,gas }}^{\text {rign }}\end{array}$ \\
\hline $\begin{array}{l}\text { FB2d3 } \\
\text { d }\end{array}$ & $15 * 2$ & $18 * 5.825$ & 8.074 & 6.078 & 0.1 & 0.6 & 0.4 & 0.5 \\
\hline FB1 & $32 \times 1 \mathrm{~cm}$ & $\begin{array}{l}2 * 2.25 \\
19 * 4.5\end{array}$ & 4.04 & 3.37 & 0.01 & 0.5 & 0.5 & 0.5 \\
\hline FB2 & $32 \times 1$ & $\begin{array}{l}31 \times 1, \\
2,3,4,10 \times 5\end{array}$ & 4.04 & 3.37 & 0.01 & 0.5 & 0.5 & 0.5 \\
\hline FB3 & $14 \times 2.5$ & $\begin{array}{l}2 * 2.25 \\
19 * 4.5\end{array}$ & 4.04 & 3.37 & 0.01 & 0.5 & 0.5 & 0.5 \\
\hline FB4 & $14 * 2.5$ & $\begin{array}{l}2 * 2.25 \\
19 * 4.5\end{array}$ & 4.04 & 3.37 & 0.1 & 0.5 & 0.5 & 0.5 \\
\hline FB5 & $\begin{array}{l}15 * 2.03 \\
2\end{array}$ & $18 * 5.623$ & 4.04 & 3.37 & 0.1 & 0.6 & 0.4 & 0.5 \\
\hline
\end{tabular}




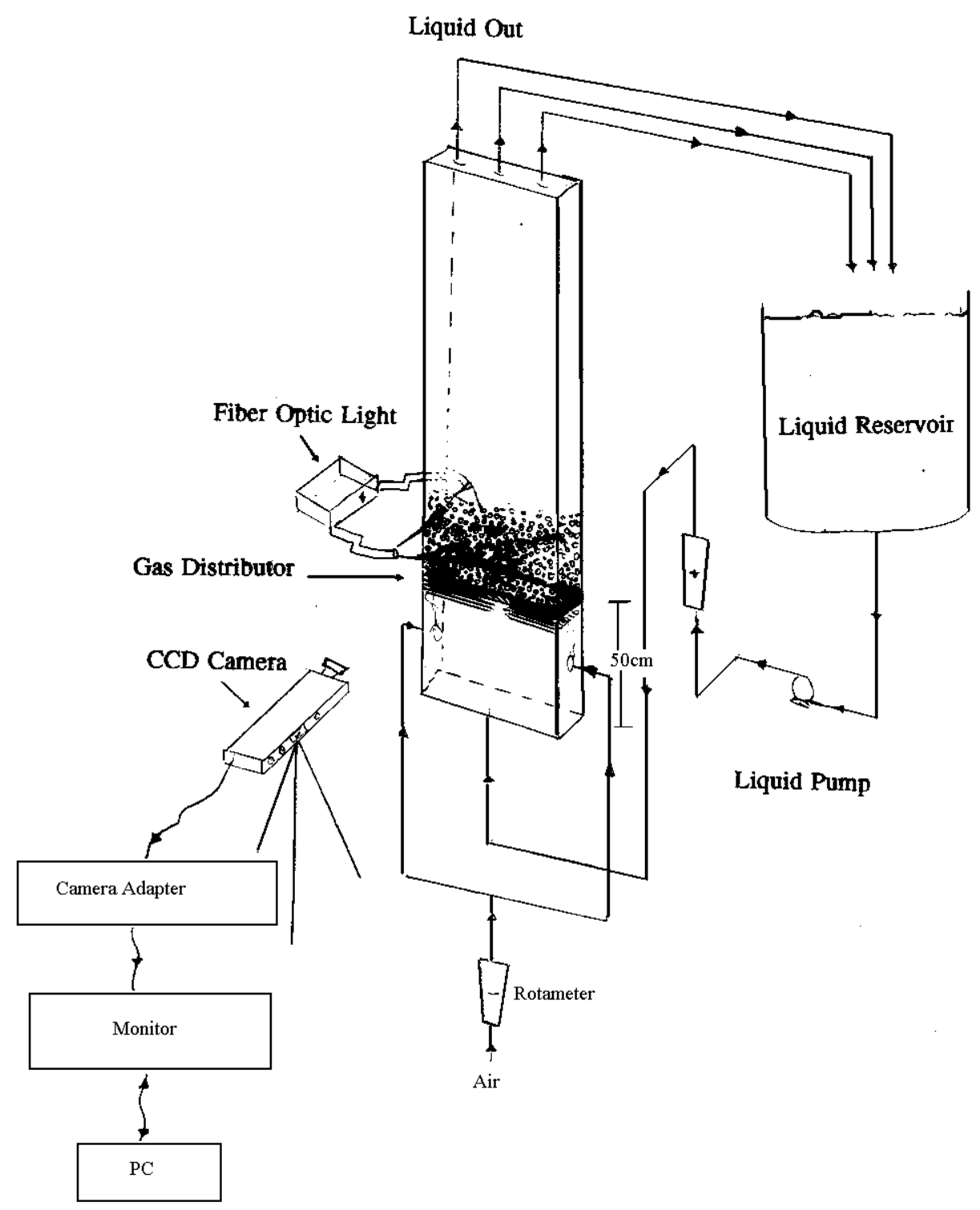

Figure 1. Experimental Schematic Diagram for Three-Phase Fluidization System. 


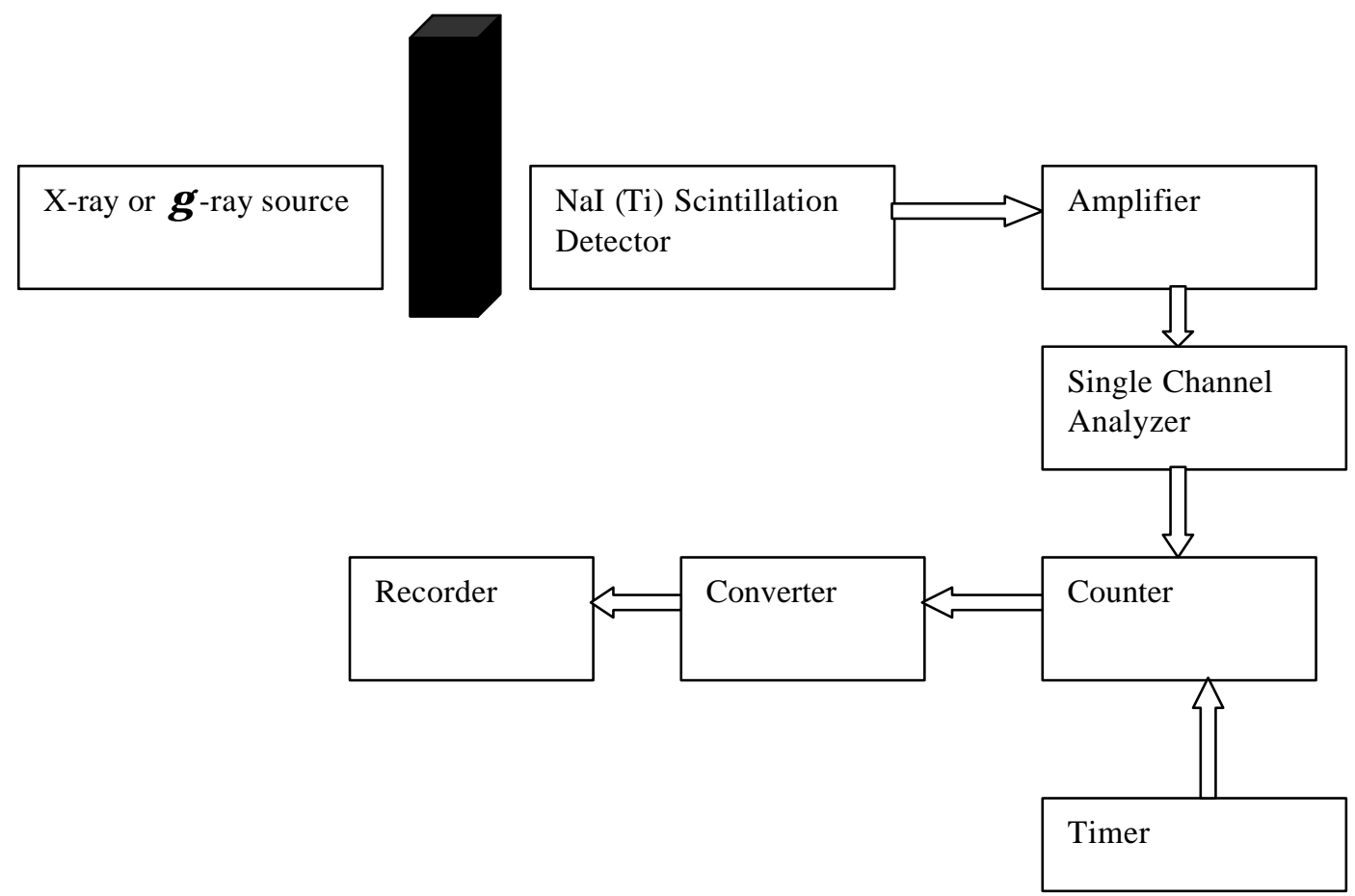

Figure 2. Schematic Diagram for Source-Detector-Recorder Assembly for X-ray and $\boldsymbol{\gamma}$-ray Densitometers. 


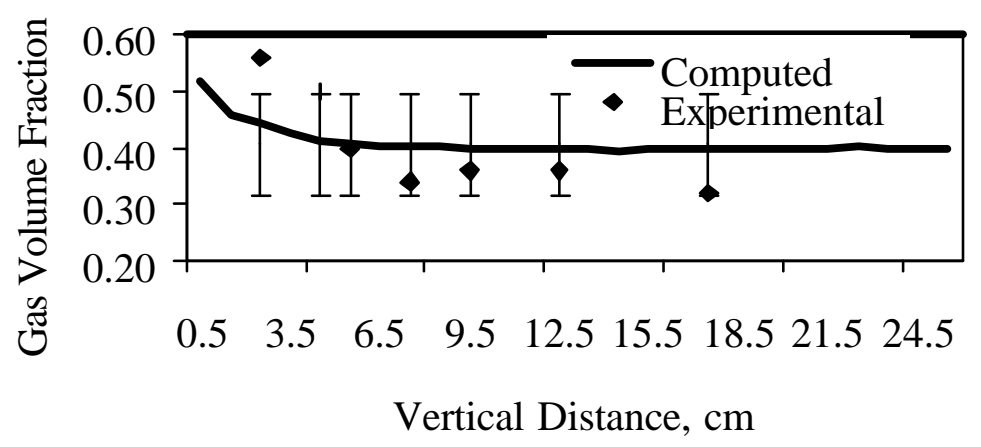

(a)

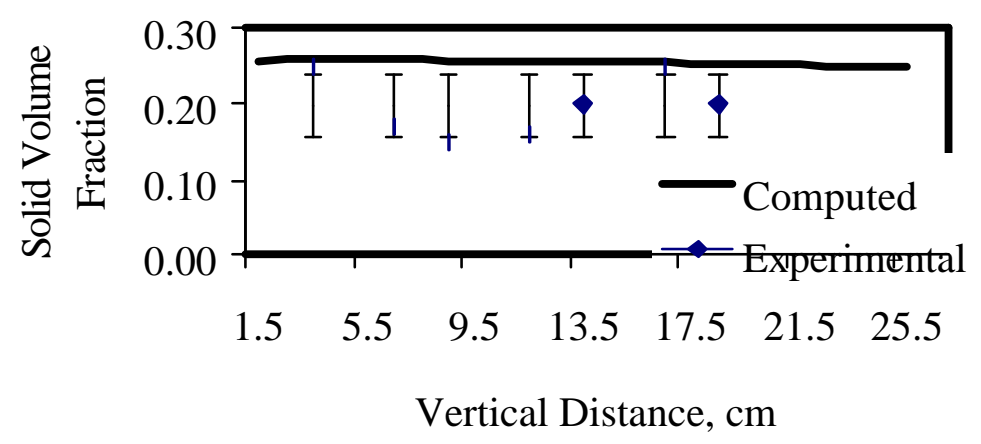

(b)

Figure 3 (a),(b). Comparison of measureu anu cumputed phase hold-up in bubbly coalesced regime for $\mathrm{V}_{\mathrm{L}}=2.04 \mathrm{~cm} / \mathrm{s}$ and $\mathrm{V}_{\mathrm{G}}=3.37 \mathrm{~cm} / \mathrm{s}$ at $4 \mathrm{~cm}$ from horizontal center of bed. 


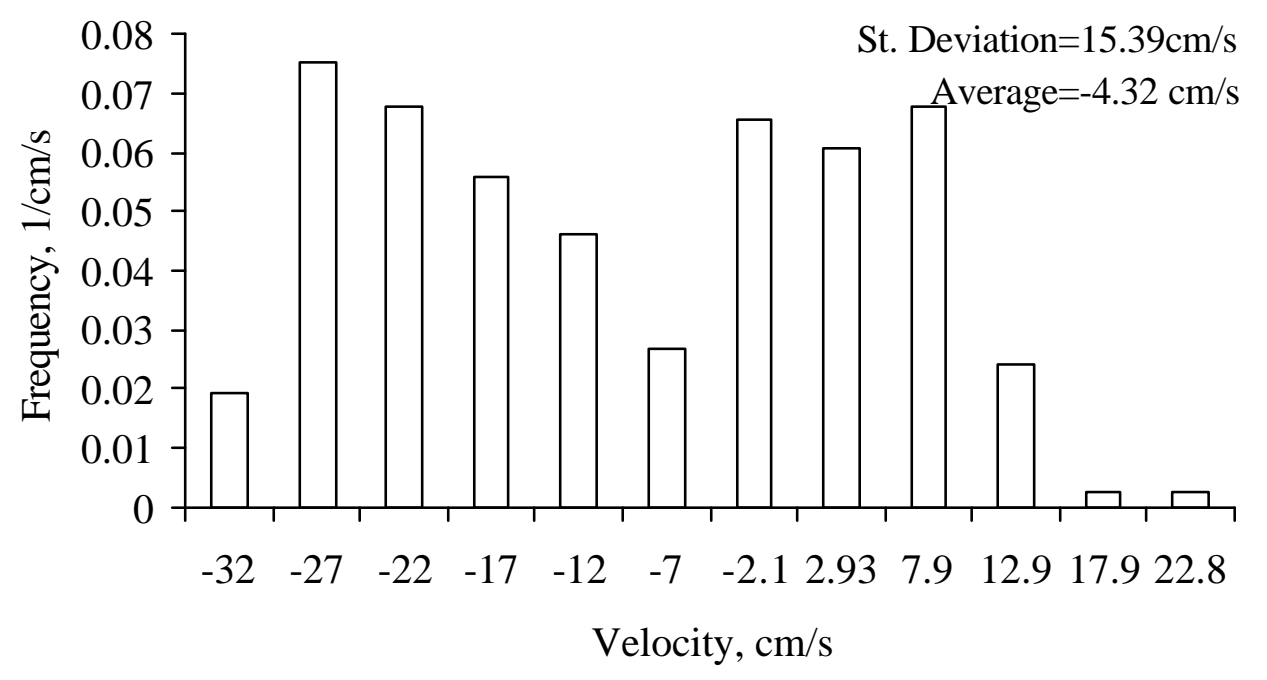

(a)

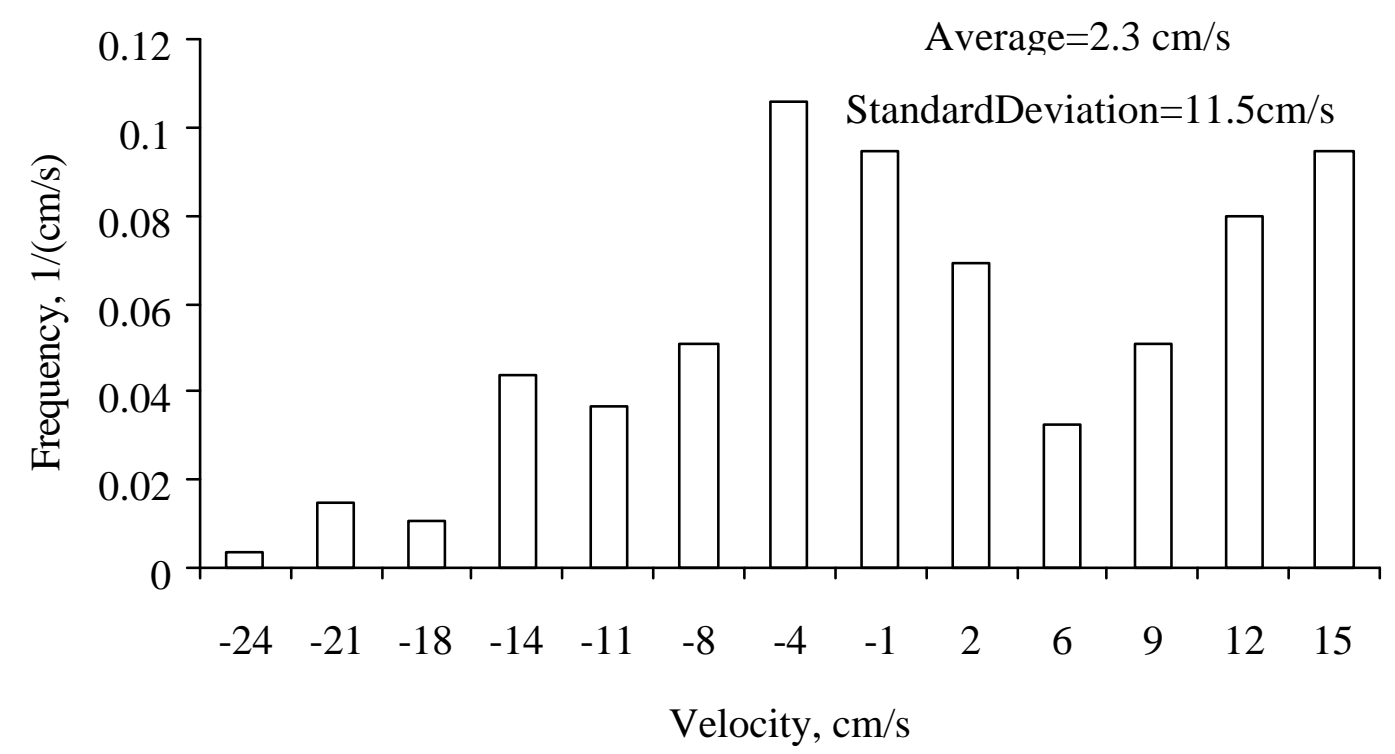

(b)

Figure 4. Experimental Vertical (a) and Horizontal (b) Particle Velocity Distribution of Solids' at a Bed Height of $9 \mathrm{~cm}$ and Horizontal Position of $7.5 \mathrm{~cm}$ from Left Wall. 


\section{$\mathrm{Y}=13 \mathrm{~cm}$ above Distributor}

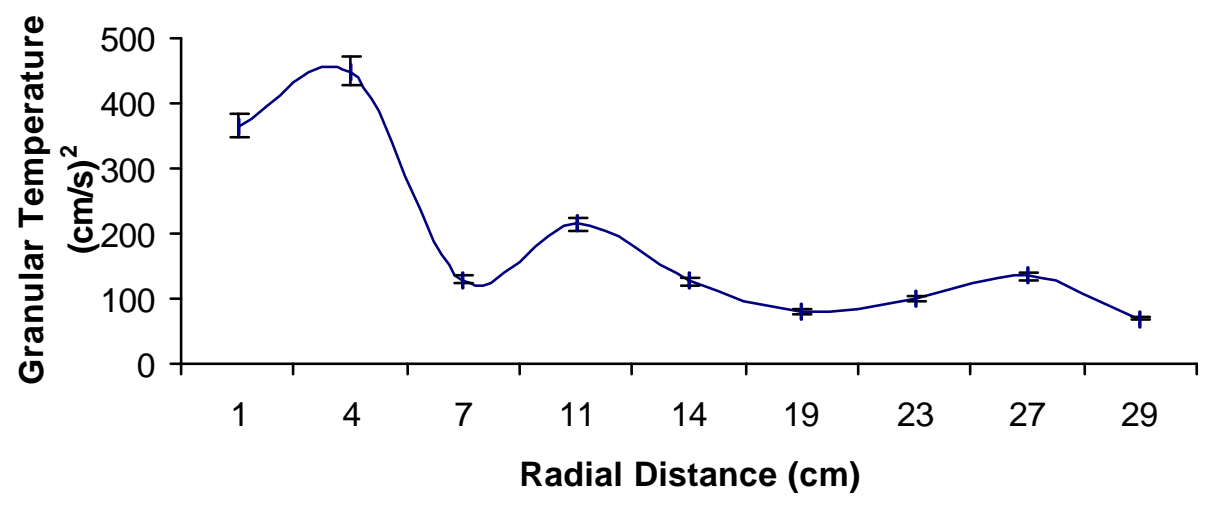

$\mathrm{Y}=7 \mathrm{~cm}$ above Distributor

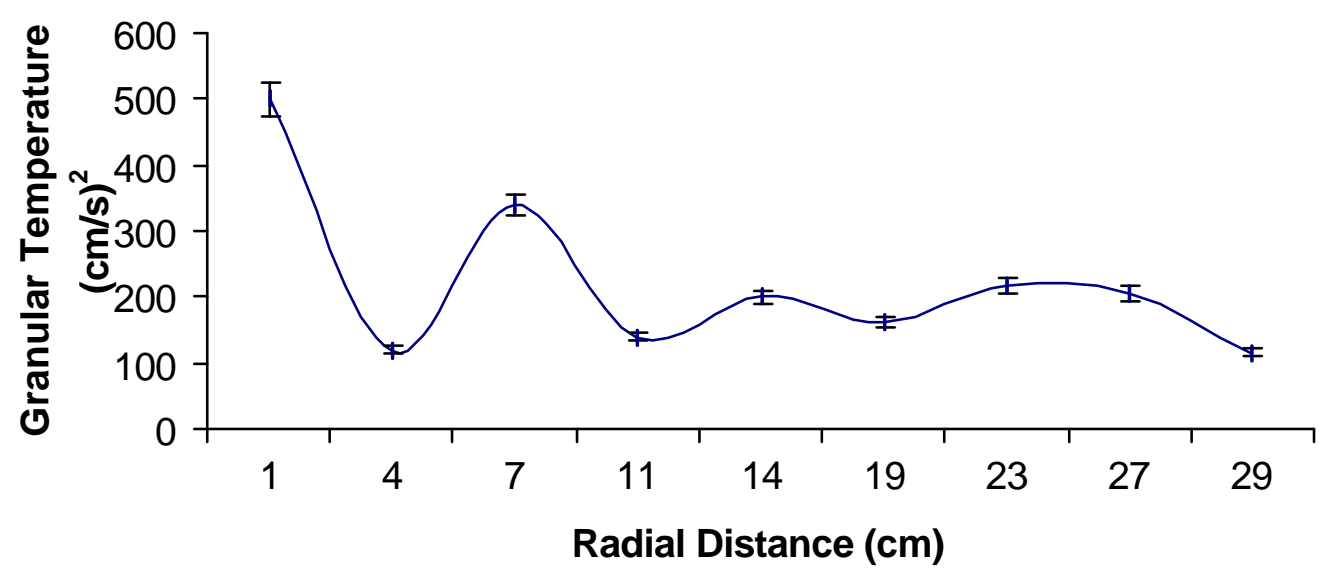

Figure 5. Granular Temperature vs. Horizontal Distance from Left Wall at Different Bed Heights in Gas-Liquid-Solid Fluidized Bed at Superficial Liquid Velocities of $4.04 \mathrm{~cm} / \mathrm{s}$ and $3.36 \mathrm{~cm} / \mathrm{s}$, respectively. 


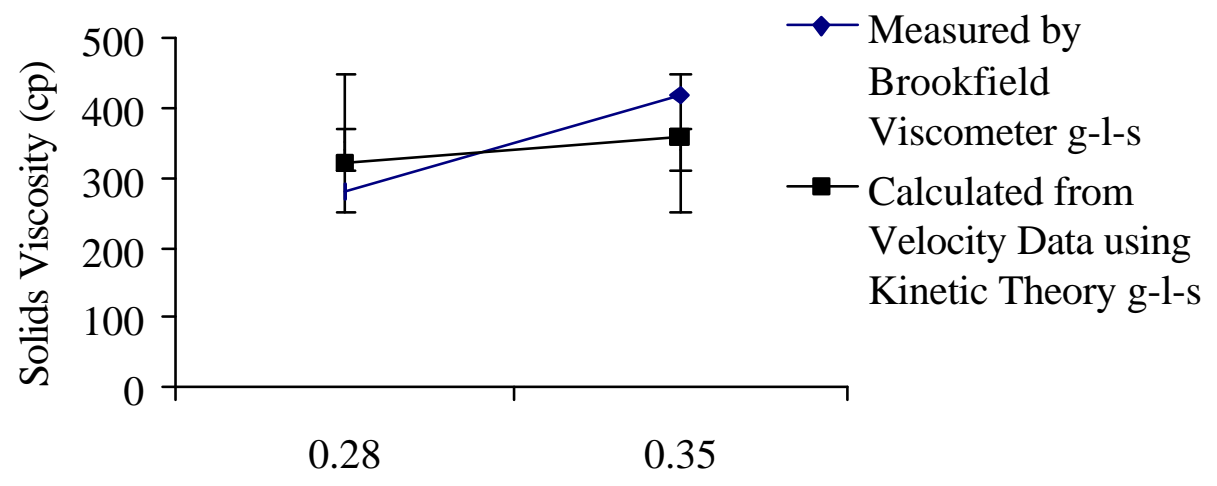

Solids Volume Fraction

Figure 6. Experimaental viscosities determined with a Brookfield Viscometer and from measurement of random particle oscillations using PIV. 


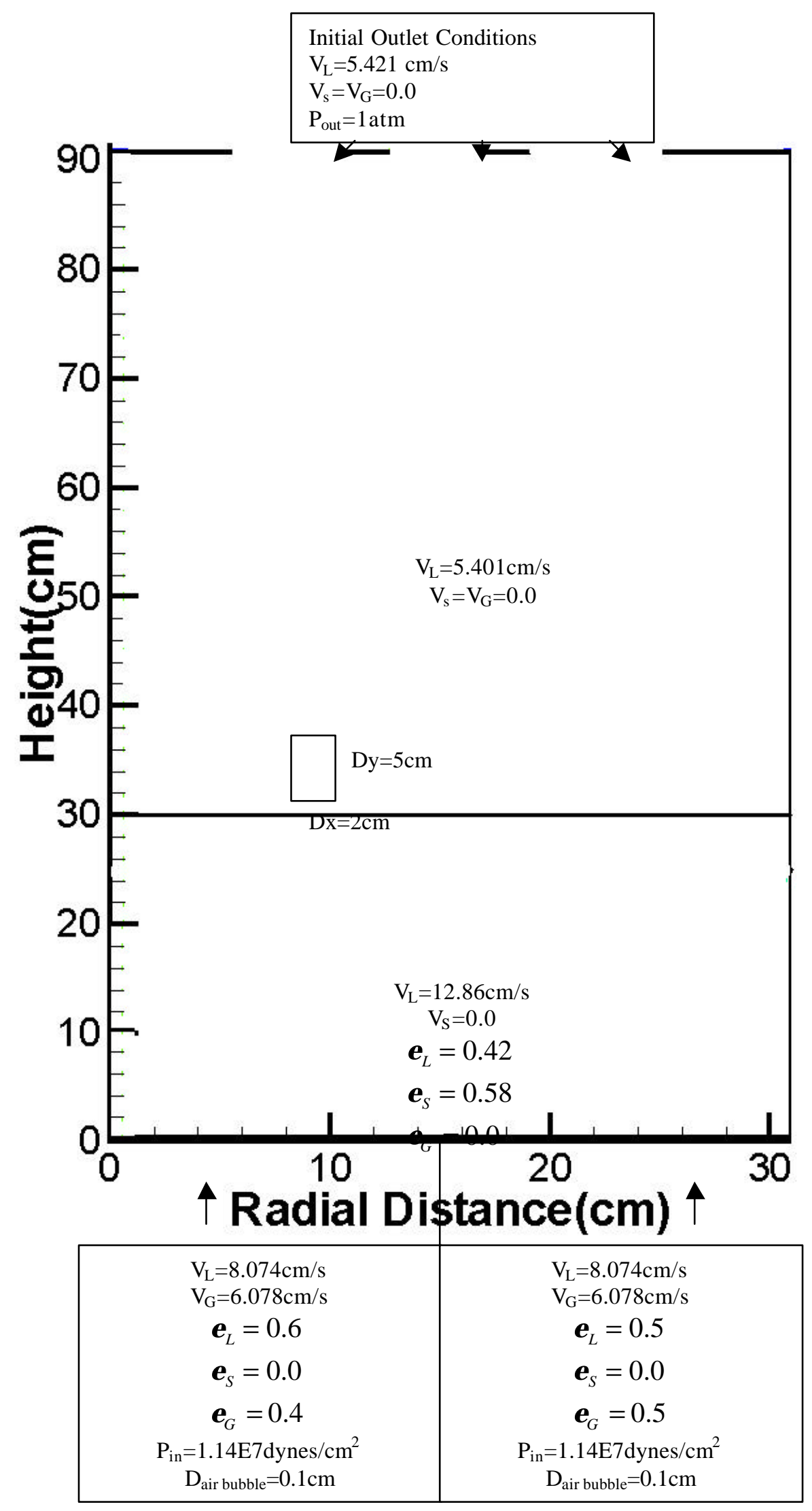

Figure 7. Inlet and initial conditions for simulations. 


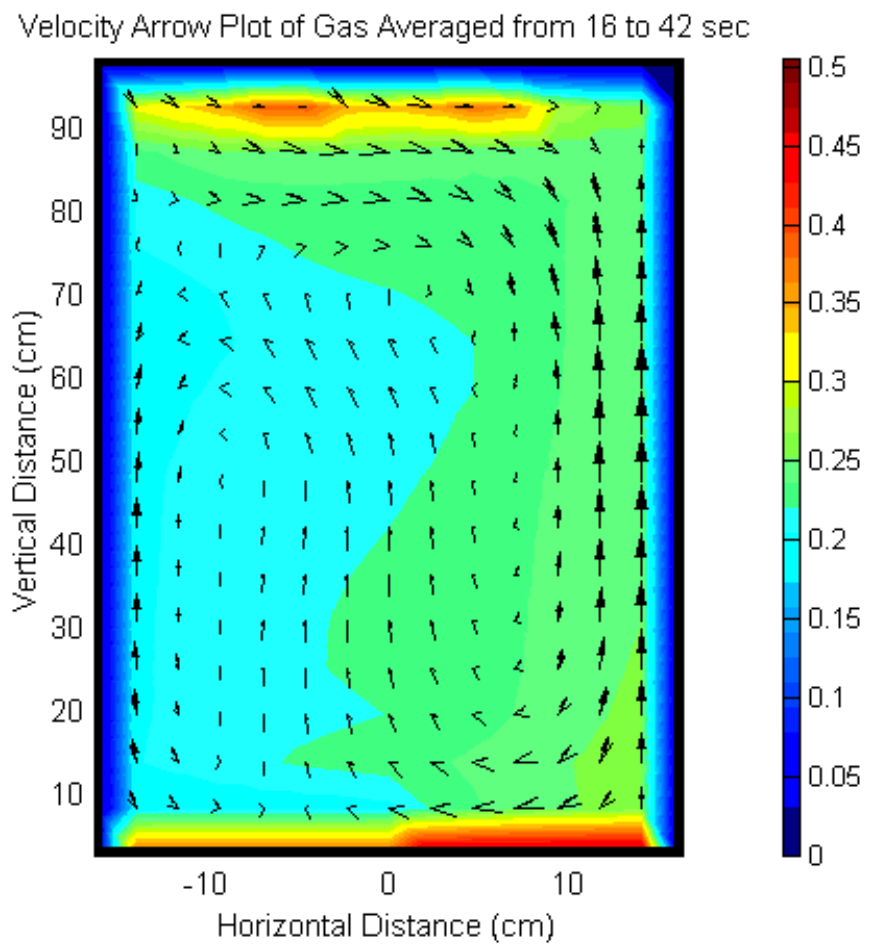

Velocity Arrow Plot of Solid 2 Averaged from 16 to $42 \mathrm{sec}$
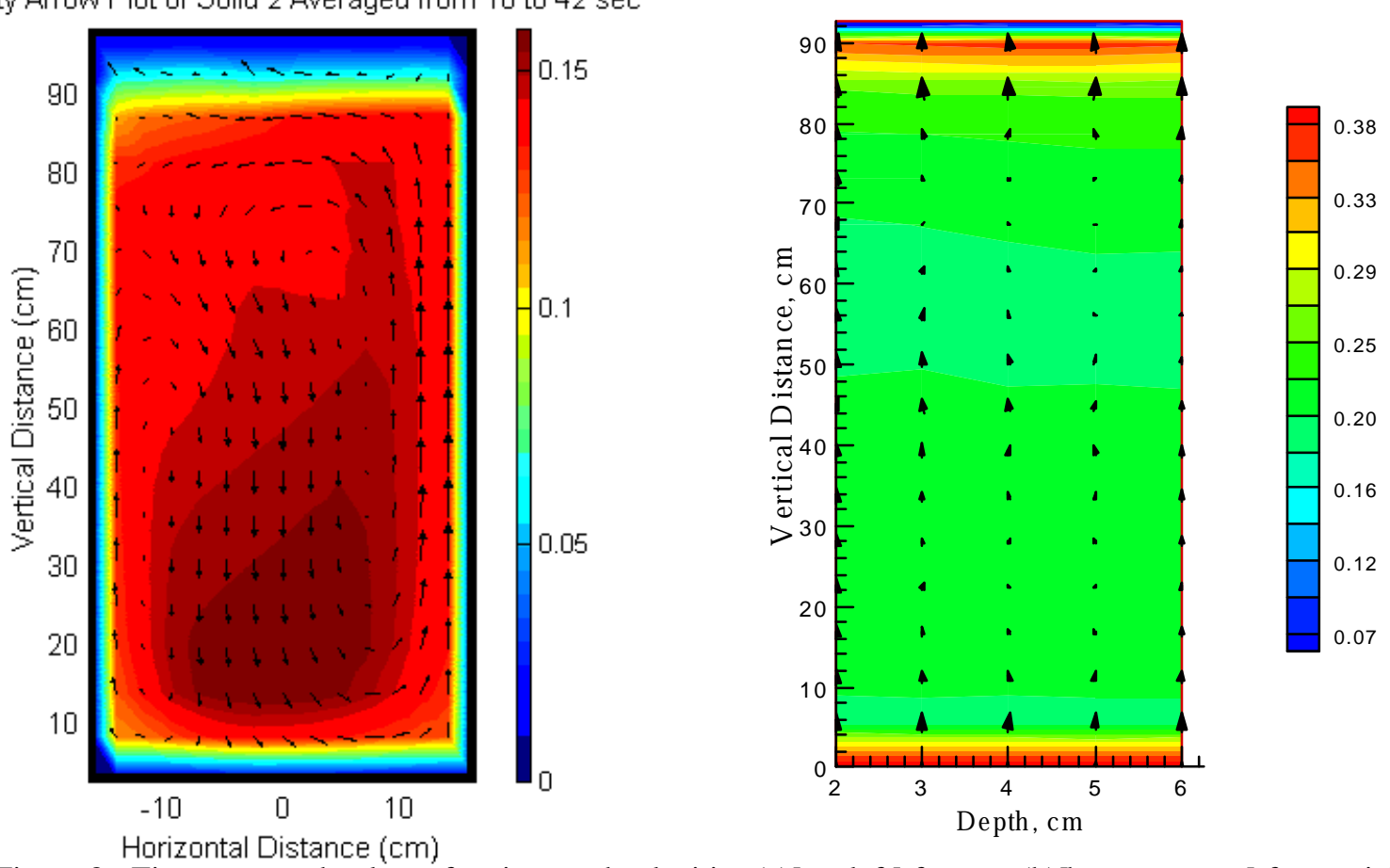

Figure 8. Time-averaged volume fractions and velocities (a)[top,left] for gas, (b)[bottom,center] for particles and 3 $\mathrm{cm}$ from front wall (c)[right] for particles. 


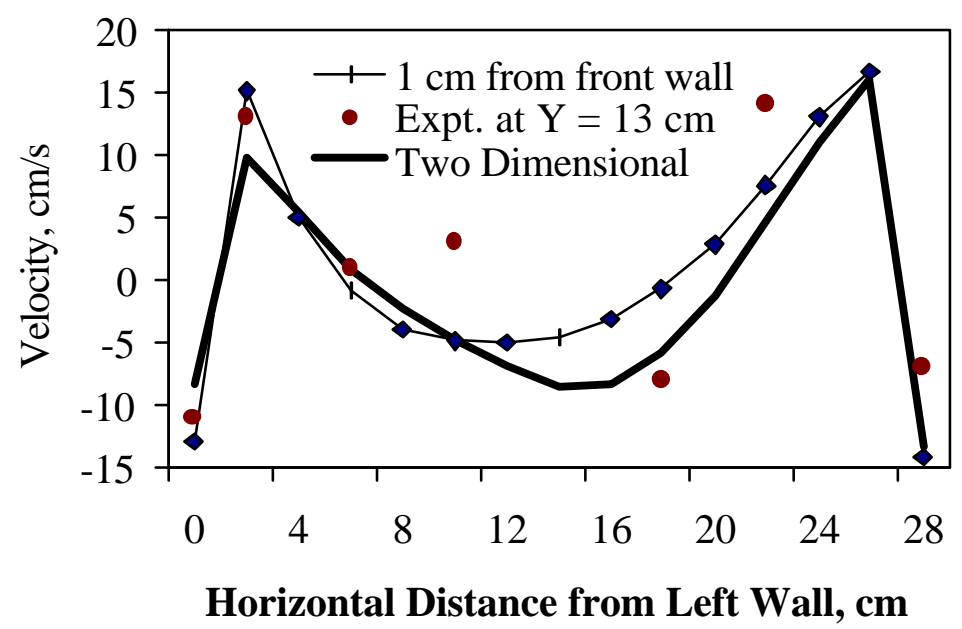

Figure 9. A comparison of two and three-dimensional vertical particle velocities to PIV measurements.

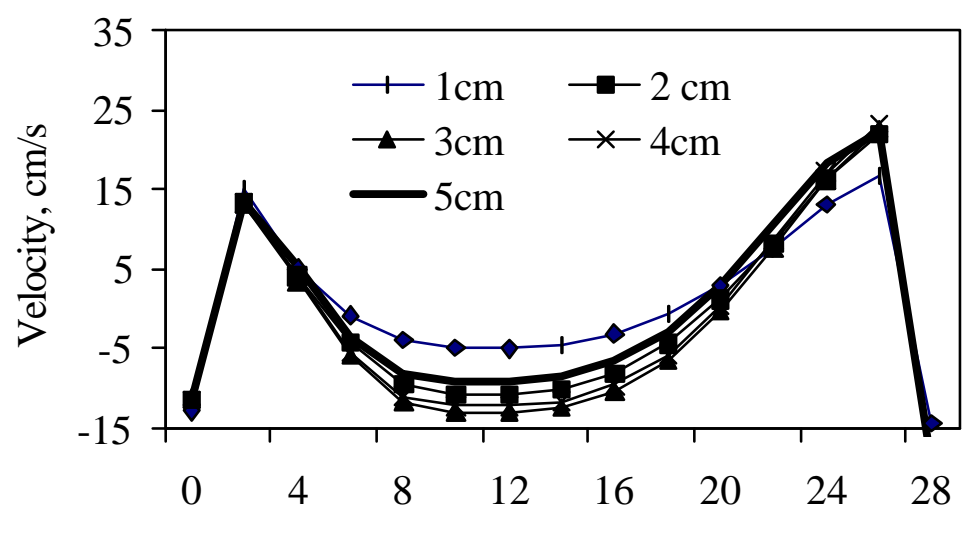

Horizontal Distance from Left Wall, cm

Figure 10. Time-averaged vertical particle velocities for various depths from front plate. 


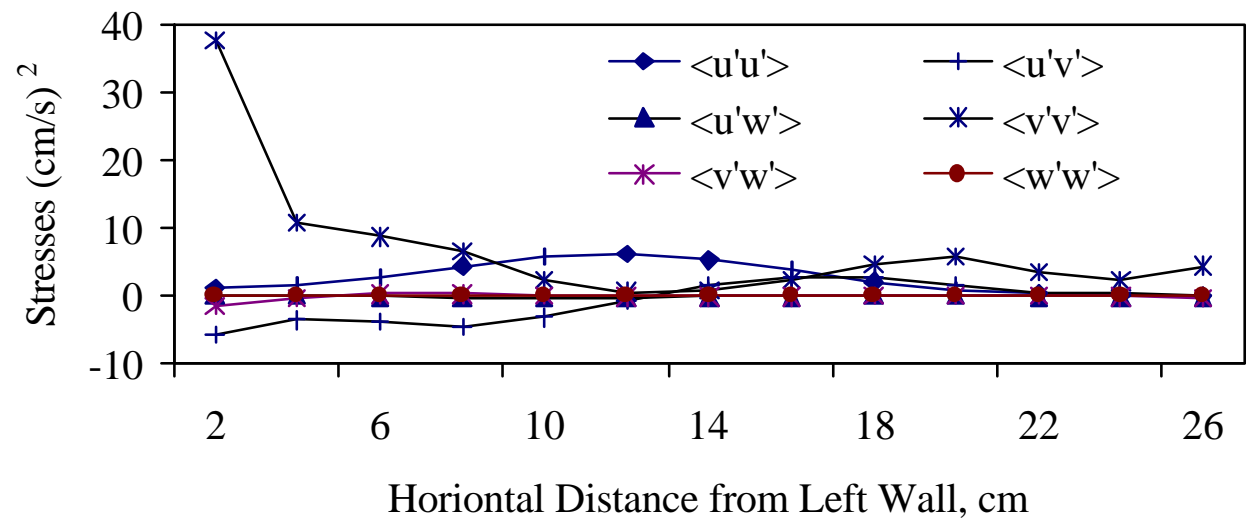

Figure 11. A comparison of the time-averaged verical velocities for gas, particles and liquid at the center of the vessel.

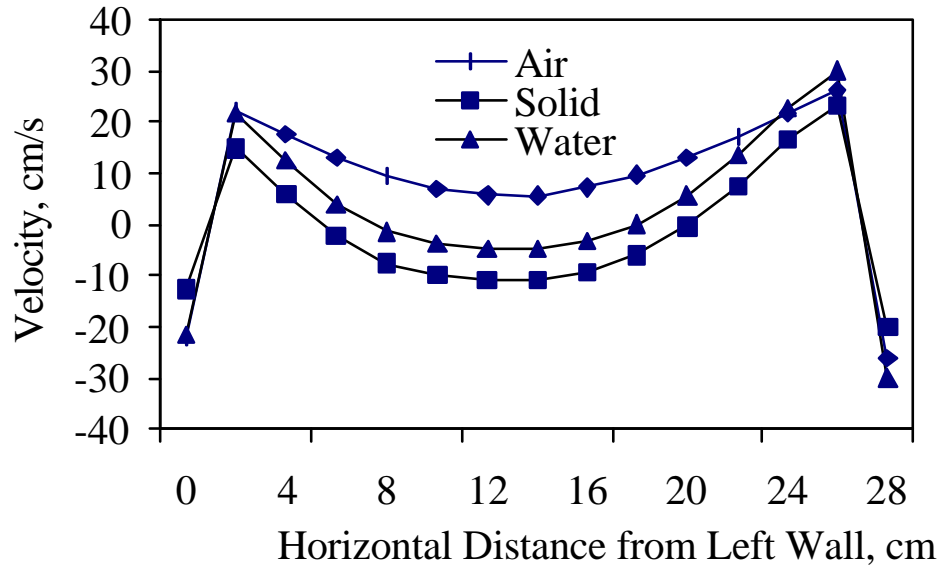

Figure 12. Typical computed Reynolds' stresses for particles at a bed height of $11.3 \mathrm{~cm}$. 


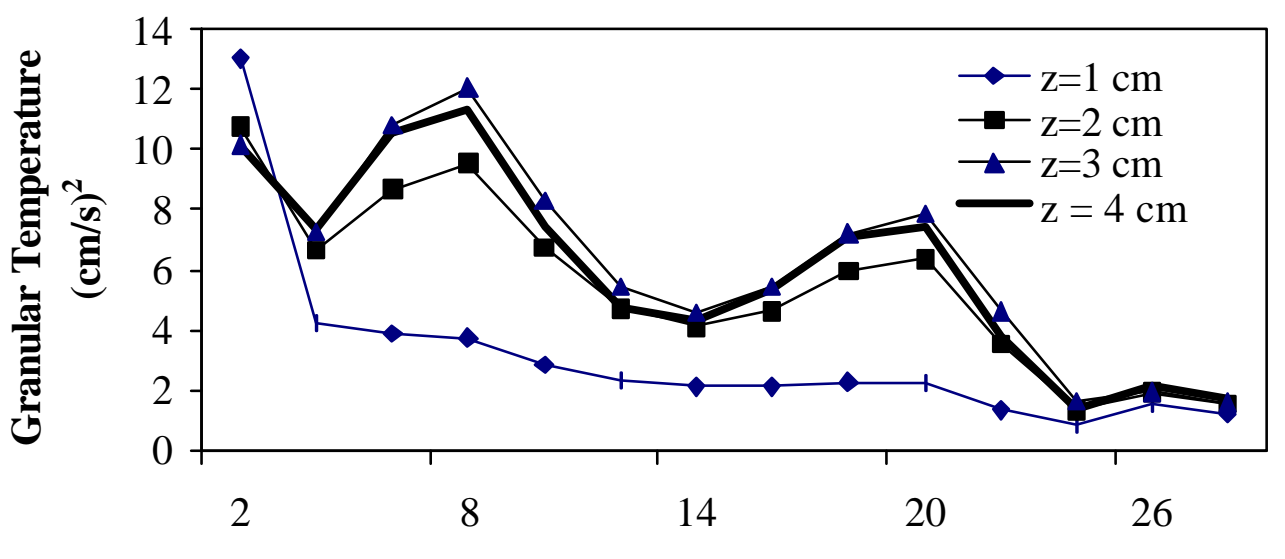

Horizontal Distance from Left Wall, cm

Figure 13. Granular Temperature (3/2 particle random kinetic energy) at several bed depths.

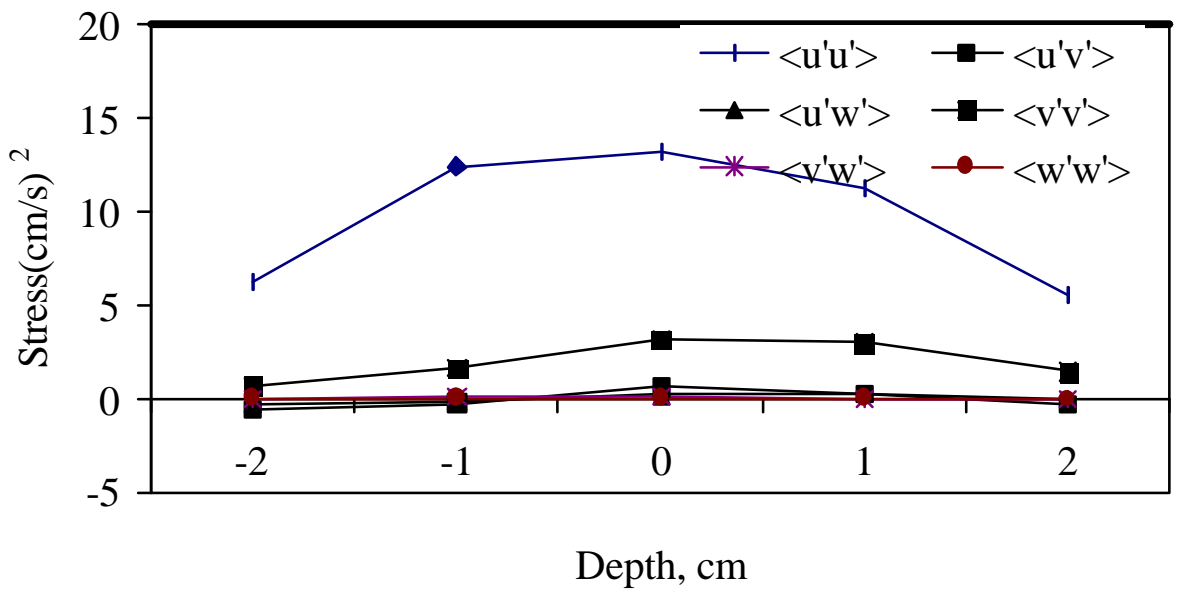

Figure 14. Variation of Reynolds stresses with depth at a bed height of $11.3 \mathrm{~cm}$ and $12 \mathrm{~cm}$ from Left Wall. 


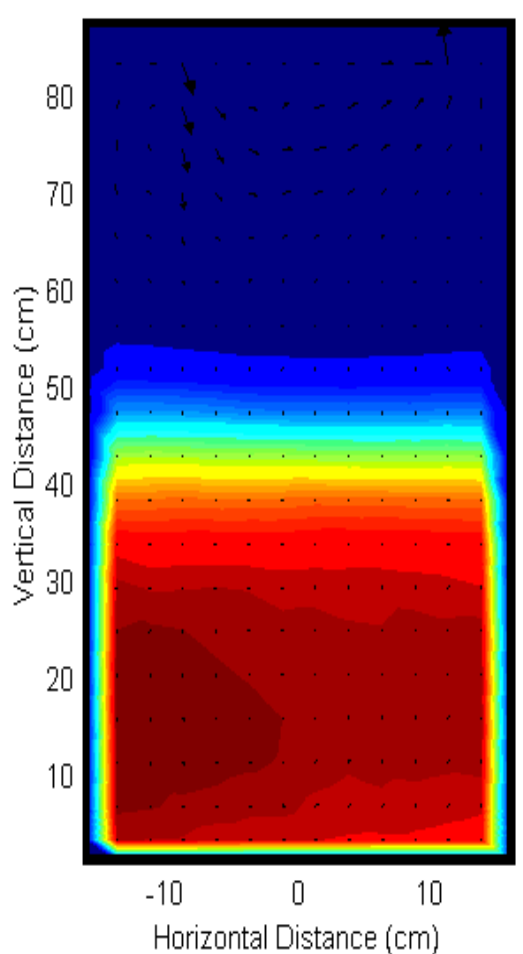

Figure 15. Particle contour and velocity vector plot for Case FB5.

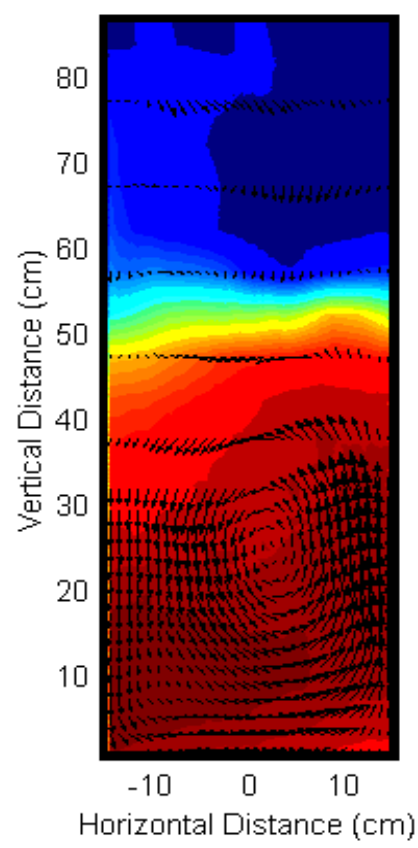

$16 \mathrm{~b}$

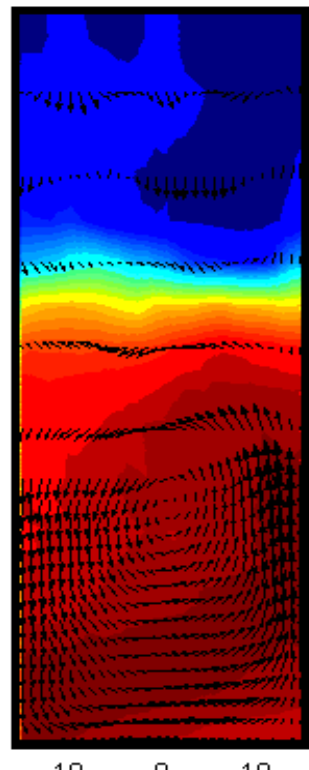

$-10$
0.3

$-0.25$

$-0.2$

$-0.15$

$-0.1$

0.05

$-10$

10

Horizontal Distance (cm)

$16 \mathrm{a}$
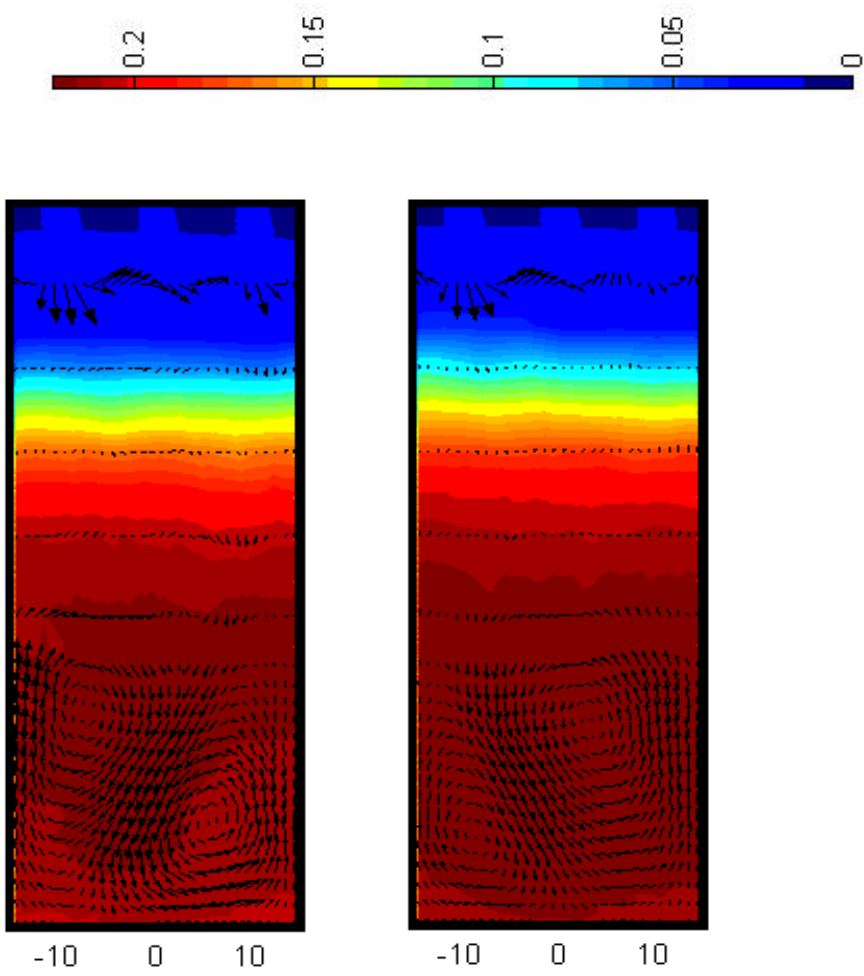

$\begin{array}{lll}-10 & 0 & 10\end{array}$

$16 \mathrm{e}$

Figure 16. (a) Time-averaged and instantaneous time, (b) $14 \mathrm{~s}$ (c) $15 \mathrm{~s}$ (d) $25 \mathrm{~s}$ (e) $28 \mathrm{~s}$ volume fraction contour and velocity vector plots for Case FB2 with the corresponding colormap bar for the volume fractions values. 


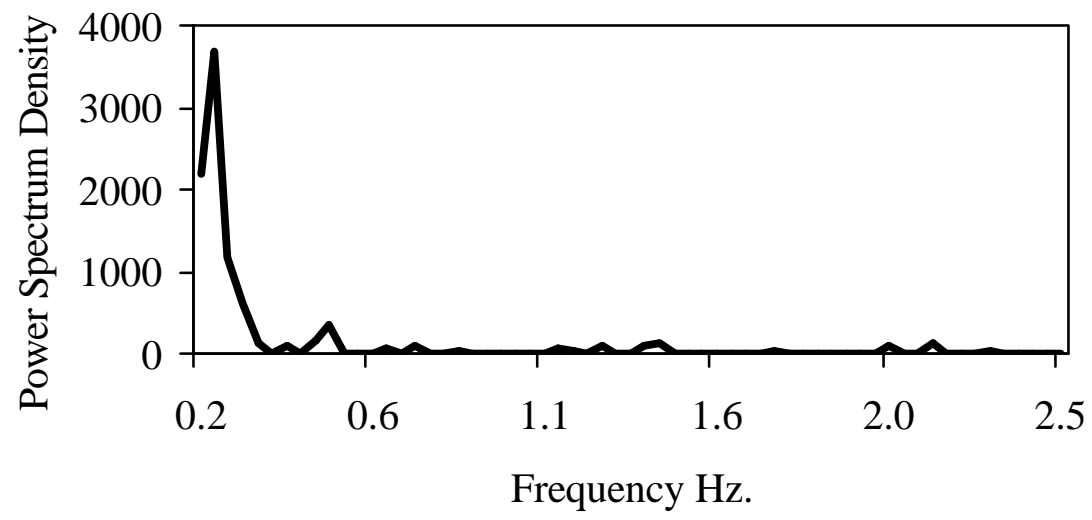

Figure 17. Power Spectrum of the Solid Axial Velocity Profile for Case FB2.

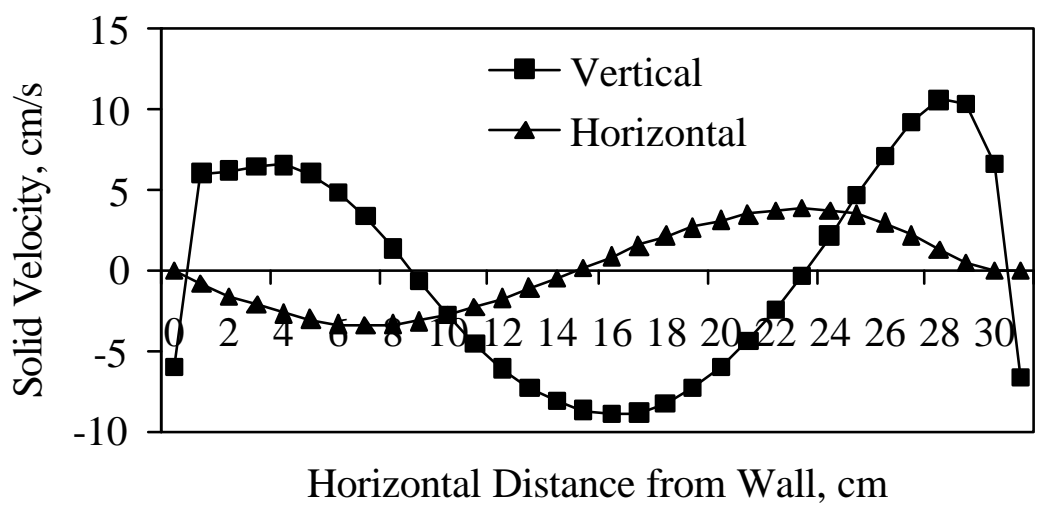

Figure 18. Time-averaged vertical and horizontal particle velocities for Case FB2 at a bed height of $9 \mathrm{~cm}$. 


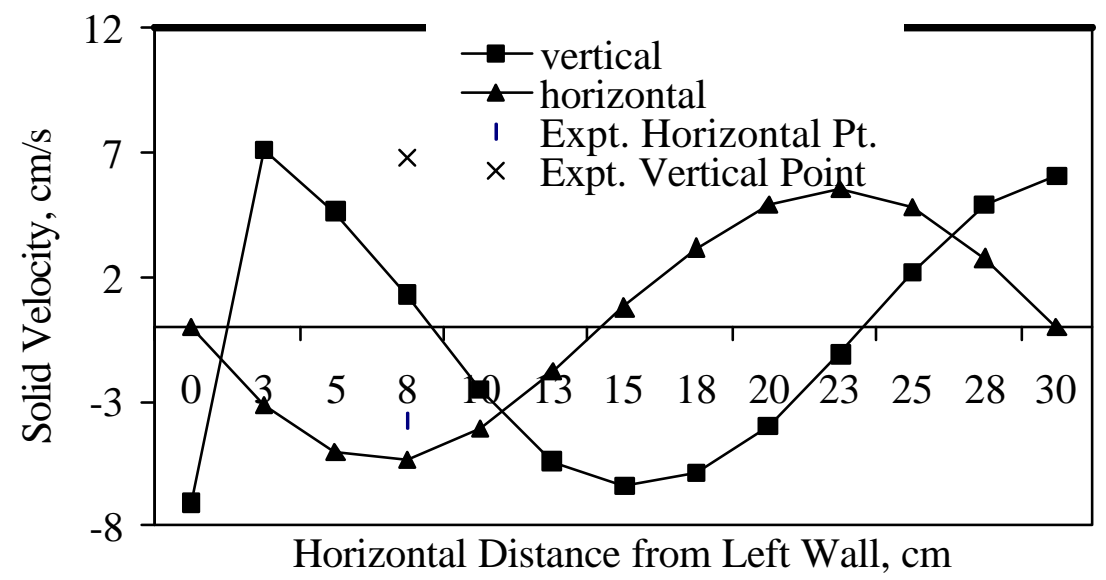

Figure 19. Time-averaged vertical and horizontal particle velocities for Case FB3 at a bed height of $9 \mathrm{~cm}$.

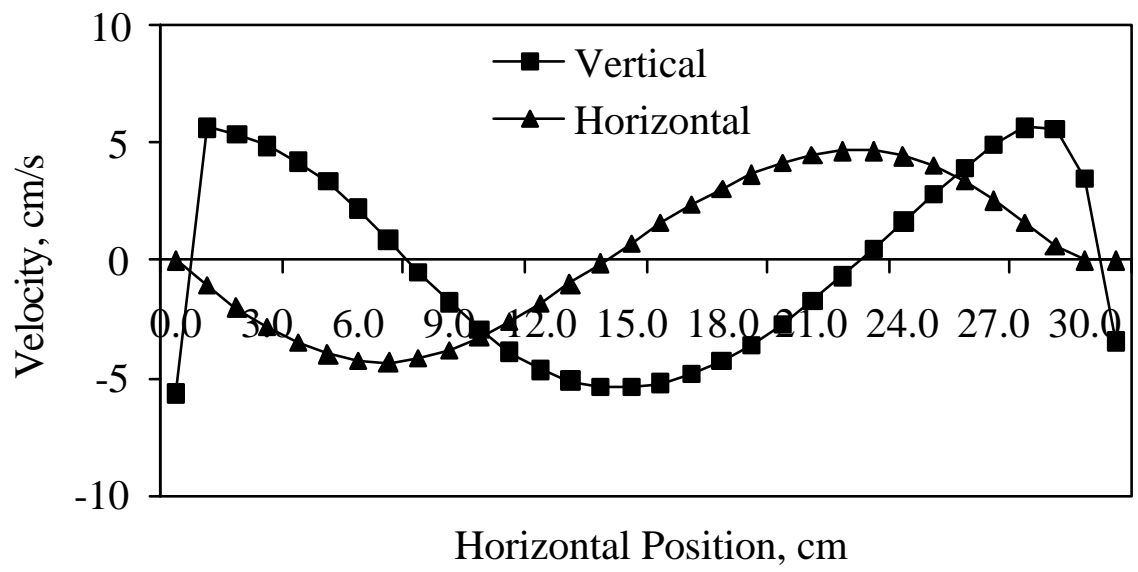

Figure 20. Time-averaged vertical and horizontal particle velocities for Case FB1 at a bed height of $9 \mathrm{~cm}$. 


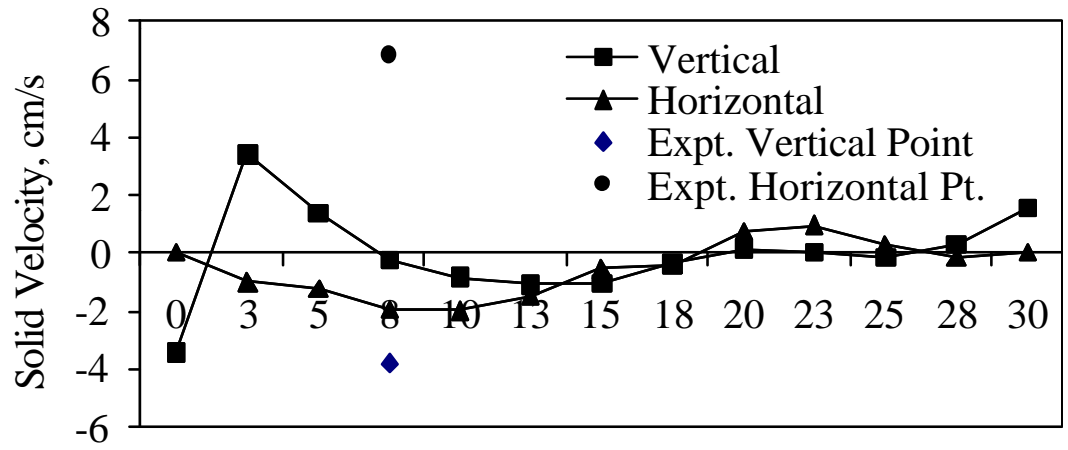

Horizontal Distance from Left Wall, cm

Figure 21. Time-averaged vertical and horizontal particle velocities for Case FB4 at a bed height of $9 \mathrm{~cm}$.

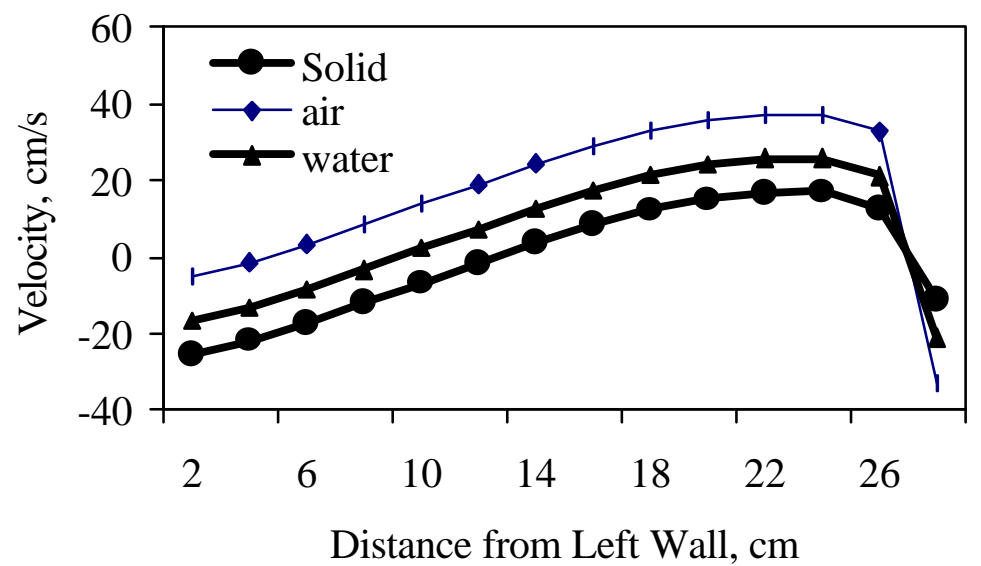

Figure 22. Time-averaged vertical and horizontal particle velocities for Case FB5 at a bed height of 9 $\mathrm{cm}$. 


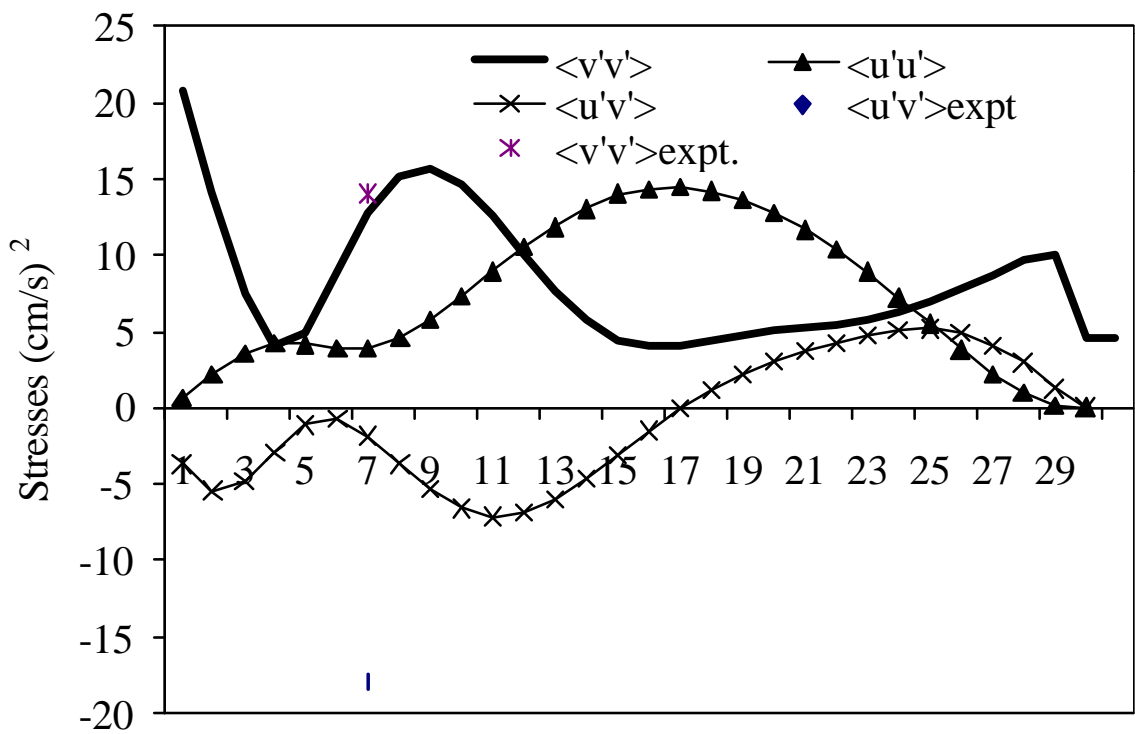

Horizontal Distance from Left Wall, cm

Figure 23. Comparison of computed normal and Reynolds shear stresses for Case FB1 and experimental points at a bed height of $9 \mathrm{~cm}$.

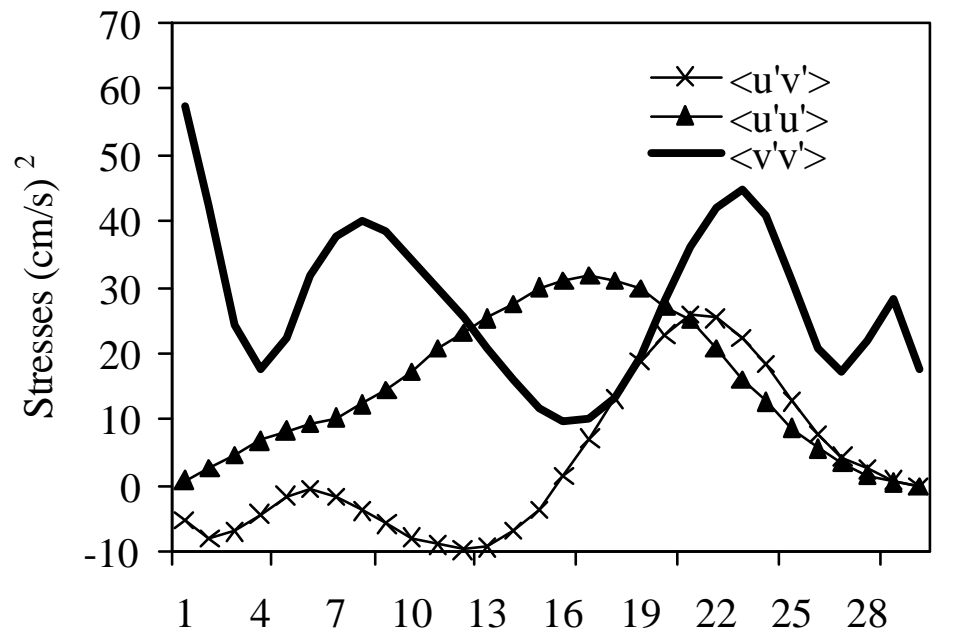

Horizontal Distance from Left Wall, cm

Figure 24. Comparison of computed normal and Reynolds shear stresses for Case FB2 at a bed height of $9 \mathrm{~cm}$. 


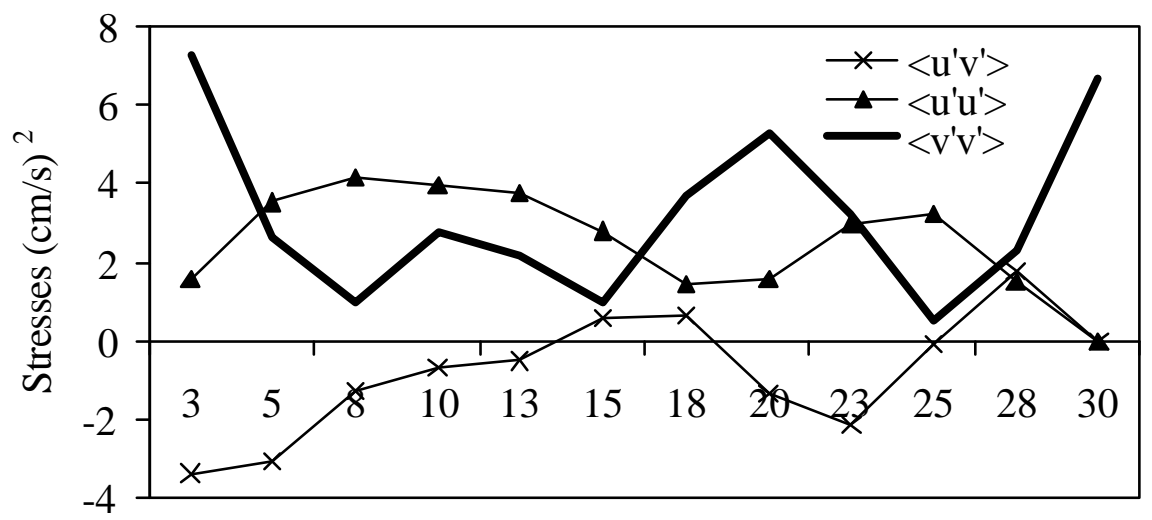

Horizontal Distance from Left Wall, cm

Figure 25. Comparison of computed normal and Reynolds shear stresses for Case FB3 and experimental points at a bed height of $9 \mathrm{~cm}$.

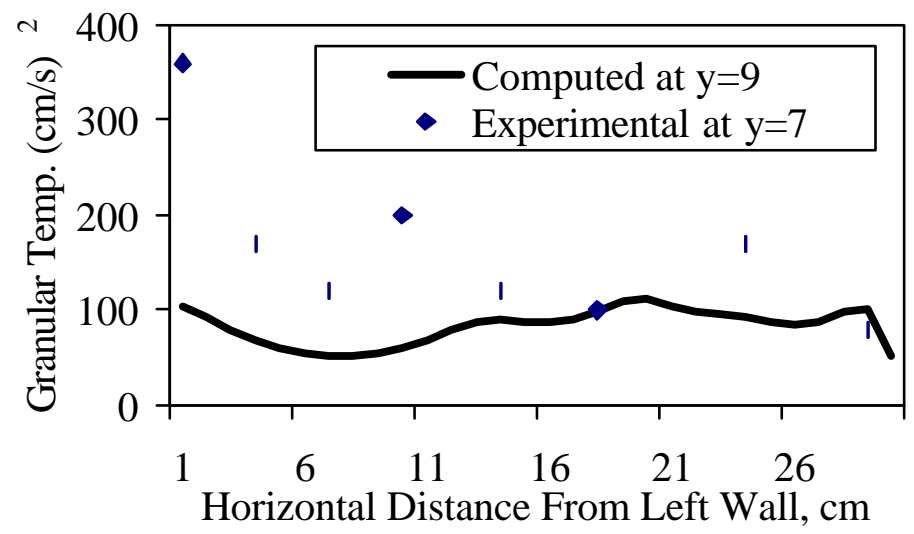

Figure 26. Comparison of computed (Case FB2) and experimental granular temperature points at a bed height of $9 \mathrm{~cm}$. 


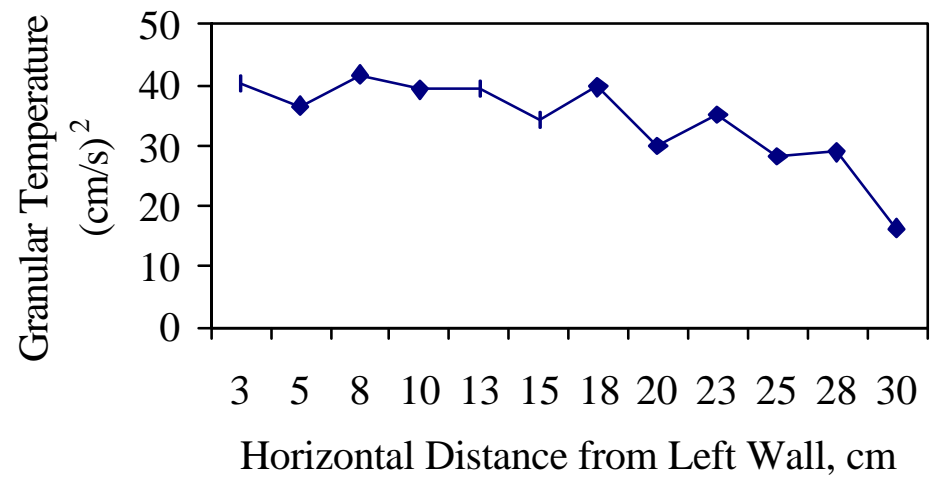

Figure 27. Comparison of computed (Case FB4) granular temperature at a bed height of $9 \mathrm{~cm}$

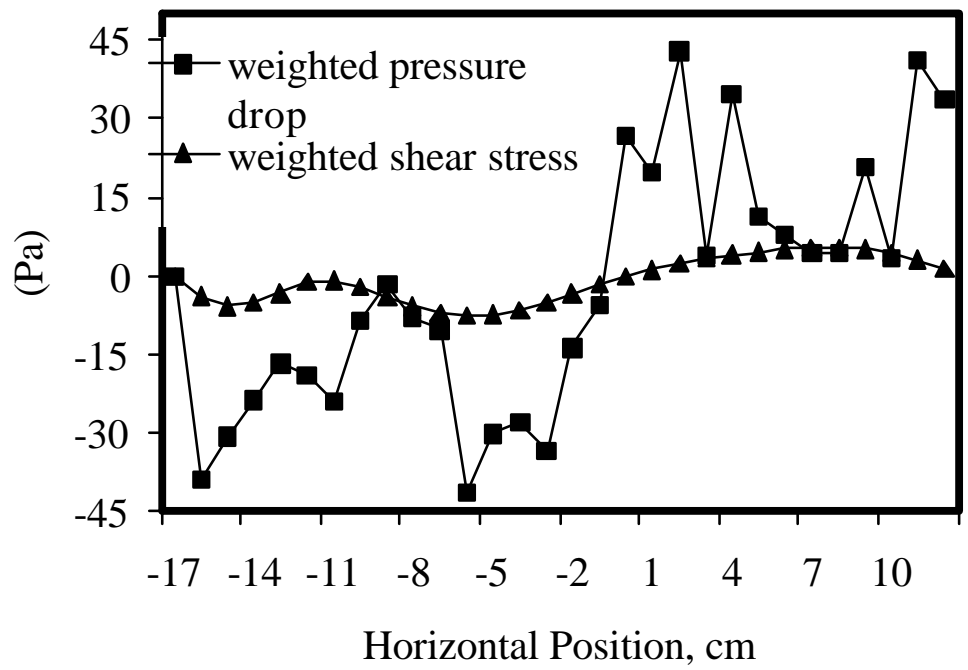

Figure 28. A test for developed flow for case FB3. 


\title{
Measurement and Computation of Turbulence in Risers
}

\section{Mehmet Tartan, Dimitri Gidaspow, Reza Mostofi, Yong Seop Shin}

\author{
Department of Chemical and Environmental Engineering \\ Illinois Institute of Technology, Chicago, IL 60616 \\ Fax: 312-567-8874, Email: gidaspow@iit.edu
}

The objective of this study is to understand turbulence in circulating fluidized beds (CFB). Tsuji, et al (1984) were the first to measure turbulent oscillations in gas-solid flow. Mudde, et al (1997) measured the turbulent stresses in a gas-liquid bubble column using PIV similar to that used here. Pan, et al (2000) used a hydrodynamic model to compute the Reynolds stresses for the data of Mudde, et al (2000). In the two-fluid approach, the use of averaged equations requires closure models. In order to improve multiphase models, such as described in Gidaspow's book (1994), a well-defined experiment is essential. Recently IIT CFB was rebuilt in order to correct the nonsymmetrical behavior caused by the elbow type outlet. Figure 1 shows the schematic diagram of IIT CFB with a splash plate type outlet. The bed material was 530 micron glass beads with a density of $2.5 \mathrm{gr} / \mathrm{cm}^{3}$. The Particle Image Velocimetry method described elsewhere (Gidaspow and Huilin, 1996) was used to measure the axial and tangential velocities of the particles. Figure 2 shows a typical streak line generated on the computer screen. This method was modified with the use of a rotating colored transparent disk. The order of the colors on the streak lines indicates the direction of the flow. Figure 3 shows the velocity distributions obtained with this method. As can be seen in this figure the distribution is close to Maxwellian. From the velocity measurements the normal and the shear stresses were computed. Also with the use of the same CCD camera a technique was developed to measure the solids volume fraction. Figure 4 shows a typical picture used for this purpose. A probe was used in these experiments to obtain a radial profile of the measured values. Figure 5 shows the schematic diagram of the setup.

Figures 6 and 7 show the solids axial and tangential velocities profiles. The axial velocity profile is approximately parabolic and symmetrical. The tangential velocity was about $1 / 50$ of the axial velocity, indicating a small rotational behavior that decreased close to the wall. The solids volume fraction profile is depicted in Figure 8. This figure shows that the riser is operating close to the core-annular regime. The solids volume fraction profile for 75 microns FCC particles (Miller and Gidaspow, 1992) shows a higher difference in the solids volume fraction between the core and annulus. The granular temperature profile is shown in Figure 9. This profile follows the solids volume fraction profile. As predicted by theory, the granular temperature is highest at the center of the tube. Figures 10 and 11 show the particle Normal and Reynolds stresses. The normal stress in the direction of the flow is approximately 10 times larger than that in the tangential direction. The $\left\langle v_{z}^{\prime} v_{z}^{\prime}\right\rangle$ is lower at the center where the $\left\langle v_{\theta}^{\prime} v_{\theta}^{\prime}\right\rangle$ is higher at that point. The Reynolds shear stress was small, producing a restitution coefficient near unity. The normal Reynolds stress in the direction of flow is large due to the fact that it is 
produced by the large gradient of velocity in the direction of flow compared to the small gradient in the $\theta$ and $r$ directions.

The table summarizes the data and computation of viscosity using two methods. The kinetic theory gives values of viscosity that agree with our previous measurements (Gidaspow, Wu and Mostofi, 1999). The values of viscosity obtained from pressure drop minus weight of bed measurements agree at the center of the tube. Particle velocities and concentrations were also measured as a function of time, as shown in Figures 13 and 14. Their principal frequency is almost one Hertz. See Fig 15 and 16. The variation of granular temperature with time is shown in Fig 17. The spectrum is in Fig18.

Preliminary computations, using model B in Gidaspow's book (1994) show a core-annular regime for the 530 microns glass beads with solids viscosity of $5.0 \times \varepsilon_{\mathrm{s}}$ as input. The computations are similar to those of Pan, et al using a CFDLIB code. The particle velocities are roughly the same as the experimental values. The velocity variances also follow the experimental trends but $\left\langle v_{z}^{\prime} v_{z}^{\prime}\right\rangle$ were much higher.

Acknowledgment

This study was supported by the National Science Foundation Fluid, Particulate and Hydraulic System Grant No. CTS-9610053, by the Department of Energy Grant No. DE-PS26-98FT40117 and by a gift from Dow Corning Corporation as a part of the Multiphase Fluid Dynamics Research Consortium.

References

Gidaspow D. 1994, Multiphase flow and fluidization: Continuum and kinetic theory descriptions, Boston: Academic Press Inc.

Gidaspow D. and Huilin L., 1996, Collisional viscosity of FCC particles in a CFB, AIChE. J. 42, 2503-2510.

Gidaspow D., Wu X. and Mostofi M.,1999, Turbulence of particles in a CFB and slurry bubble columns using kinetic theory, AIChE annual meeting preprints, Fluidization and Fluid-Particle systems, Dallas, 261-266.

Miller A. and Gidaspow D.,1992, Dense, vertical gas-solid flow in a pipe, AIChE. J. 38, 1801-1815.

Mudde R.F.,Lee J.,Reese J. and Fan L.S.,1997, Role of coherent structures on Reynolds stresses in a 2-D bubble column, AIChE. J. 43, 913.

Pan Y., Dudukovic M.P. and Chang M.,2000,Numerical investigation of gas driven flow in 2-D bubble columns, AIChE. J. 46, 434-449.

Tsuji Y., Morikawa Y. and Shiomi H.,1984, LDV measurements of an air-solid two-phase flow in a vertical pipe, J.Fluid Mech. 139, 417-434. 


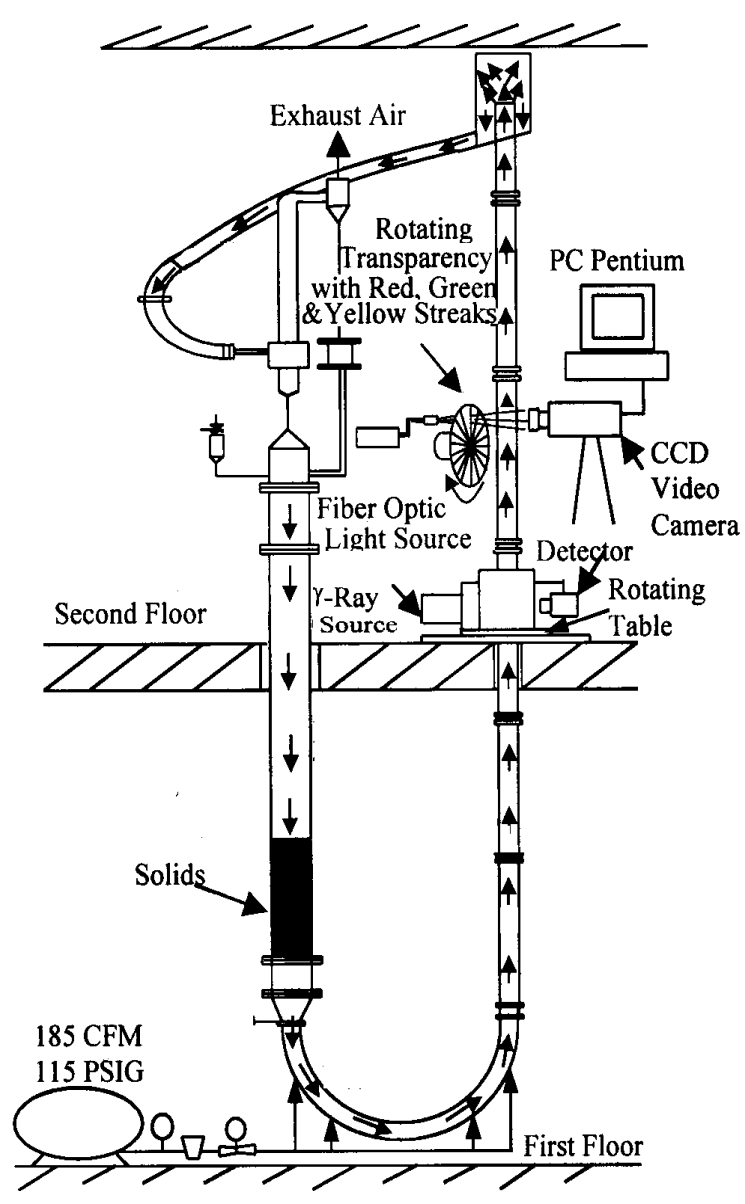

Fig. 1 IIT Circulating Fluidized Bed with Splash Plate

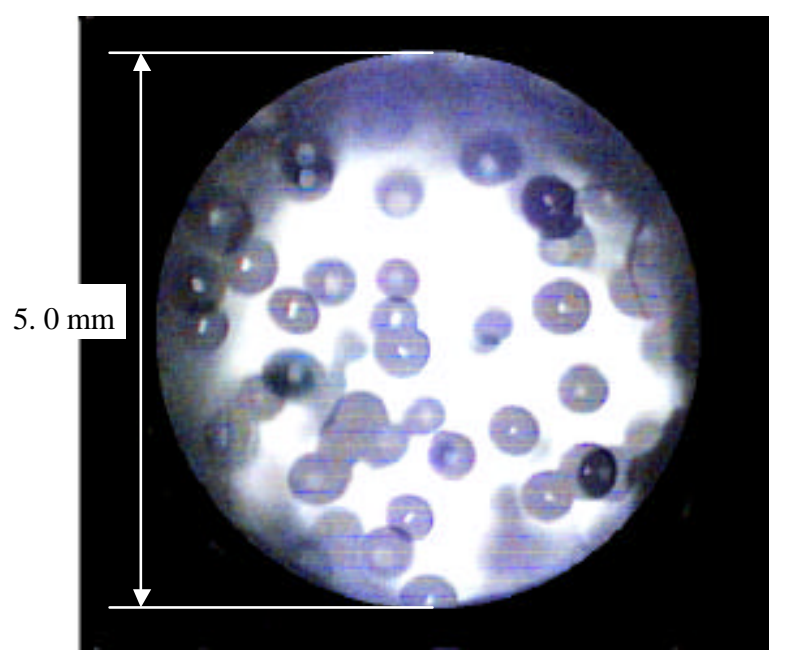

Fig. 4 Particle Image View Through CCD Camera $\mathrm{d}_{\mathrm{p}}=530 \mu \mathrm{m}$, Control Volume Height : $9 \mathrm{~mm}$

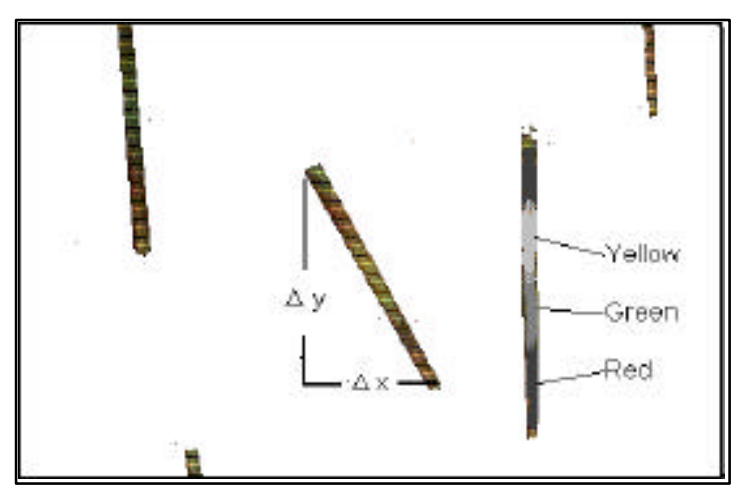

Fig. 2 Typical streak images captured by the CCD camera, $\mathrm{d}_{\mathrm{p}}=530 \mu \mathrm{m}$

(Exposure time $=0.001 \mathrm{~s}$,

Typical length $=1 \mathrm{~mm}$ )
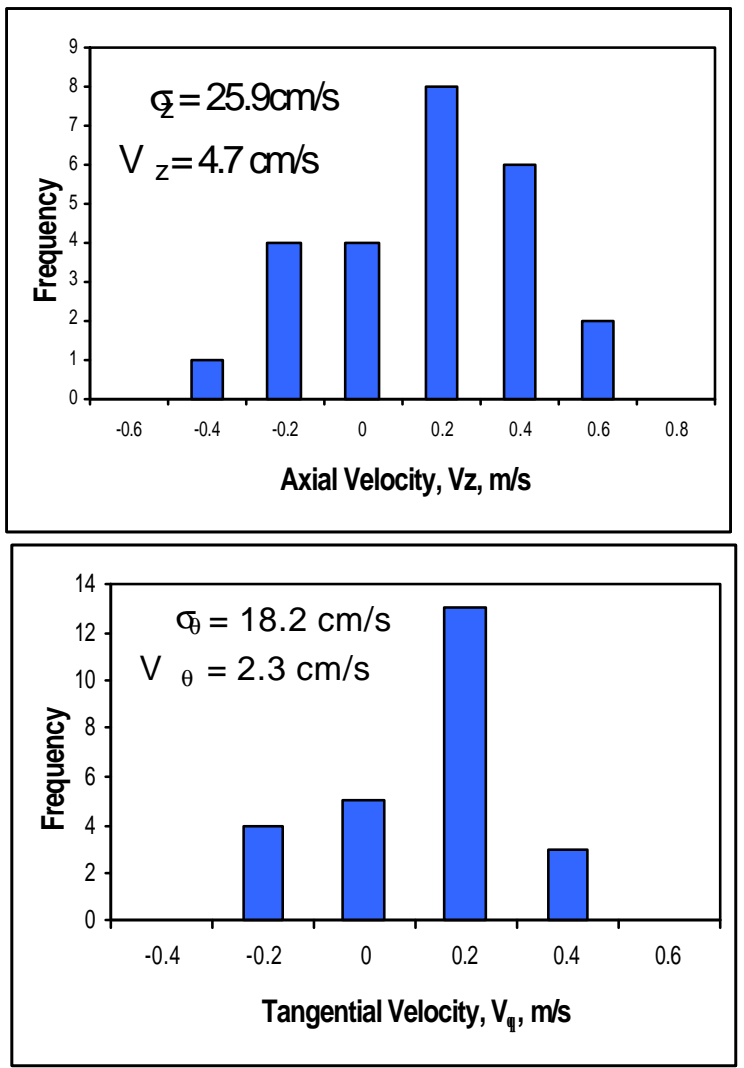

Fig. 3 Axial and Tangential Velocities, $m / s, d_{p}=530$ $\mu \mathrm{m}$,

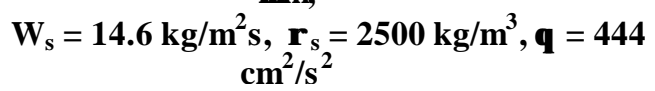




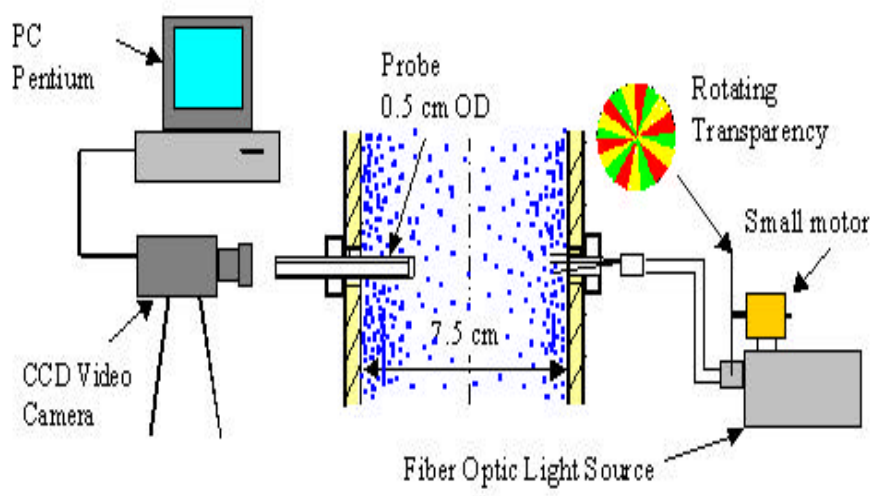

Fig.5 Particle Velocity and Solid Volume Fraction Measurements

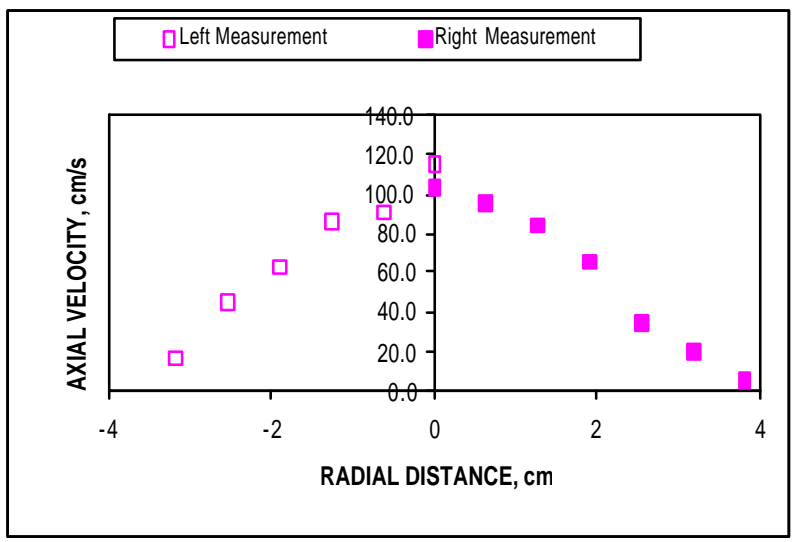

Fig.6 Particle Axial Velocity in the IIT Riser for $530 \mu \mathrm{m}$ glass beads

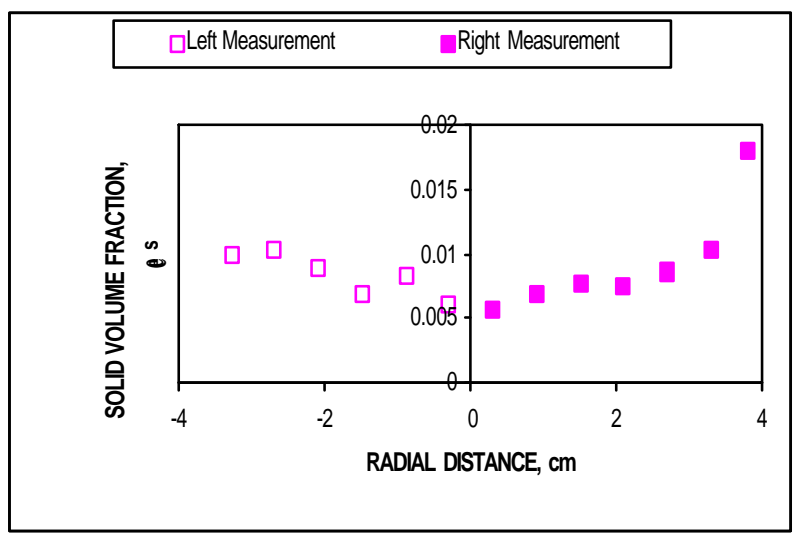

Fig.8 Particle Volume Fraction

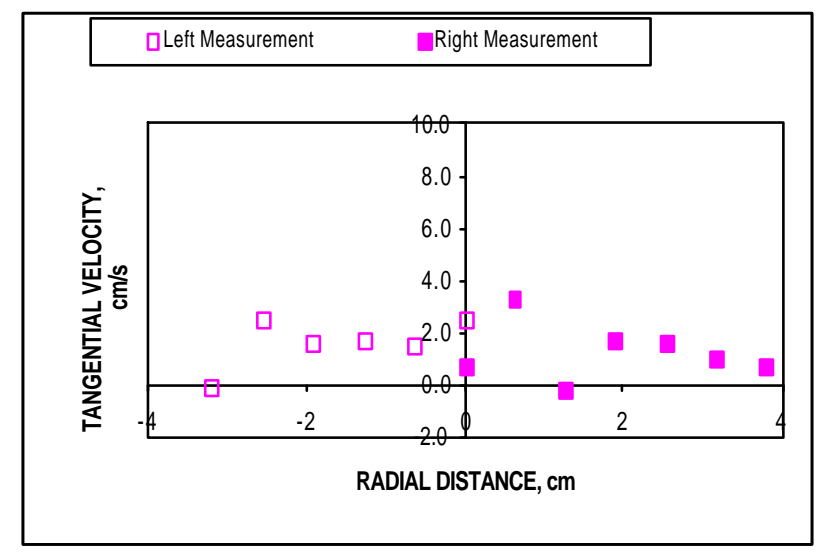

Fig.7 Particle Tangential Velocity

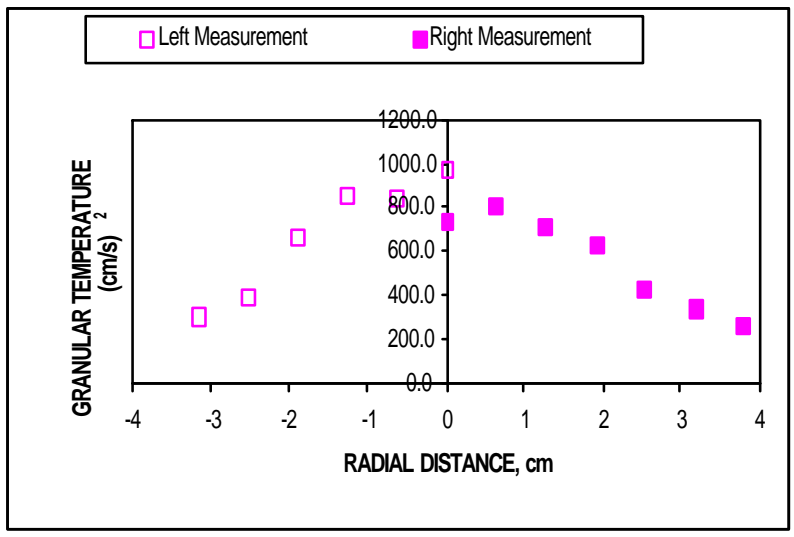

Fig.9 Granular Temperature in the IIT Riser for $530 \mu \mathrm{m}$ glass beads

$$
\begin{aligned}
& \text { Granular Temp., } \boldsymbol{\theta}=2 / 3 \sigma_{\theta}^{2}+1 / 3 \sigma_{\mathrm{z}}^{2} \\
& \sigma_{i}^{2}=\frac{1}{N} \sum_{i=1}^{N}\left(v_{i}-\bar{v}\right)^{2} \\
& \bar{v}=\text { ParticleAv erageVelocity } \\
& \mathbf{N}=\# \text { of particles in a unit volume }
\end{aligned}
$$




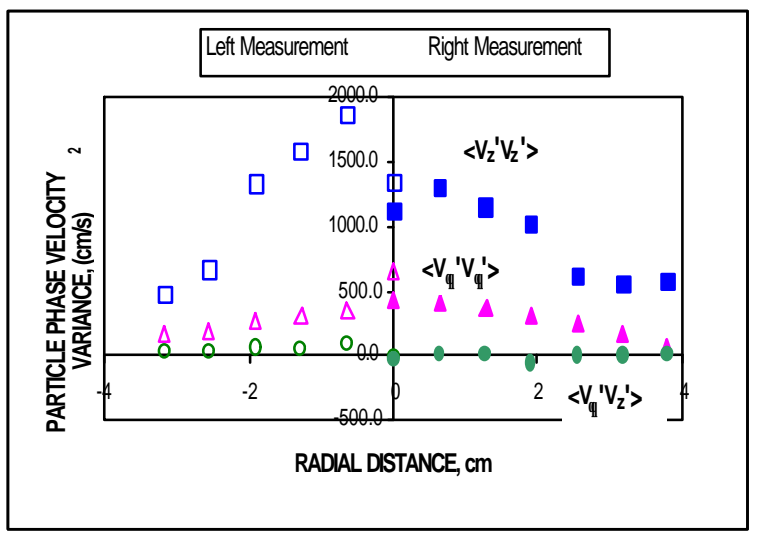

Fig. 10 Normal and Shear Reynolds Stresses

$v_{\theta}{ }^{\prime} v_{Z}{ }^{\prime}=\frac{1}{N} \sum_{i=1}^{N}\left(v_{\theta i}-\overline{v_{\theta}}\right)\left(v_{Z i}-\overline{v_{Z}}\right)$

$\mathrm{N}=$ \# of particles in a unit volume

$\left\langle v_{\theta}{ }^{\prime} v_{z}{ }^{\prime}\right\rangle=\frac{1}{T} \int_{0}^{T}\left(v_{\theta}{ }^{\prime} v_{z}{ }^{\prime}\right) d t$

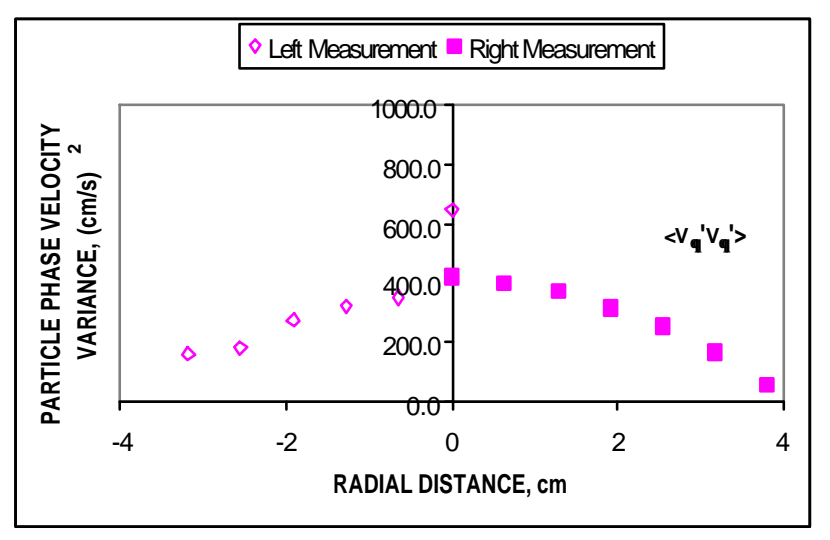

Fig. 12 Particle Phase Normal Reynolds

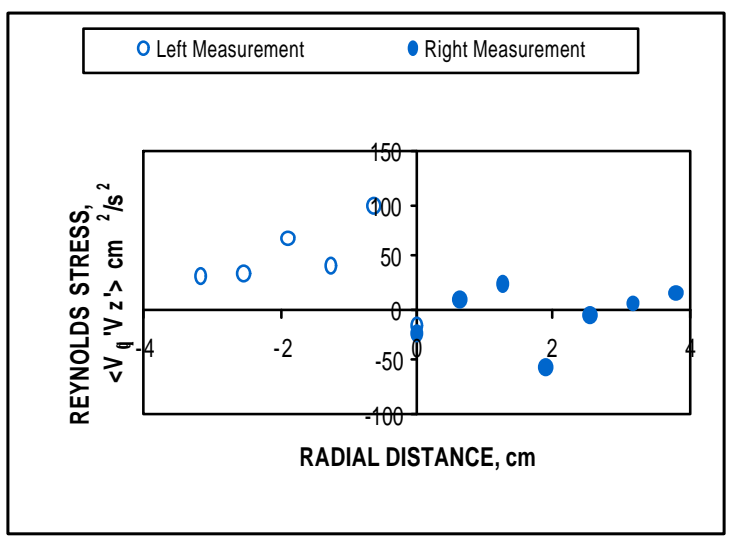

Fig. 11 Particle Phase Reynolds Stress

Solid Viscosity from Kinetic Theory:

$\mu_{s}=\frac{5 \rho_{p} d_{p} \sqrt{\pi \theta}}{48(1+e) g_{o}}\left[1+\frac{4}{5}(1+e) g_{o} \varepsilon_{s}\right]^{2}+\frac{4}{5} \varepsilon_{s}^{2} \rho_{p} d_{p} g_{o}(1+e) \sqrt{\frac{\theta}{\pi}}$

Viscosity from Mixture Momentum Equation:

$\mu=\frac{\left(-\frac{d P}{d z}\right) \frac{R}{2}-\frac{1}{R} \int_{0}^{R} \varepsilon_{s} \rho_{s} g r d r}{\left(-\frac{d V_{s}}{d r}\right)_{r=R}}$ 


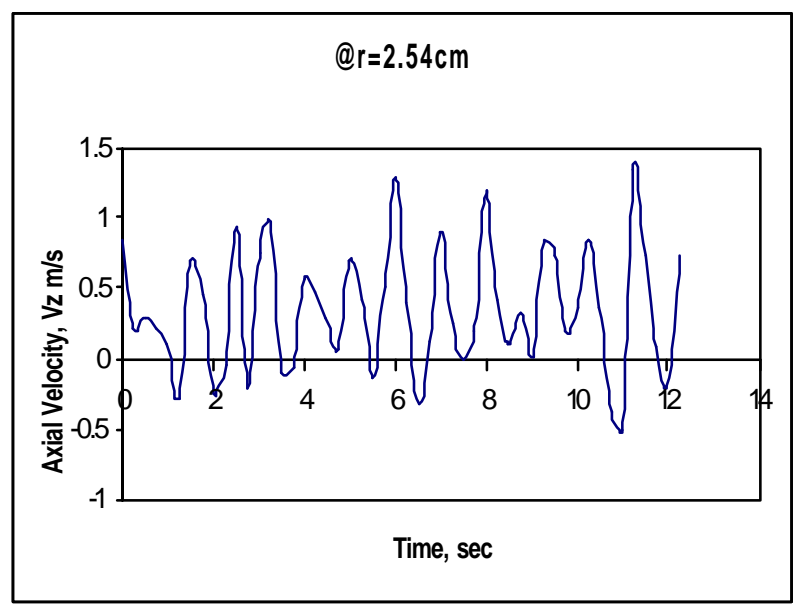

Fig. 13 Time Series of Solid Axial Velocity at $\mathrm{r}=2.54 \mathrm{~cm}$

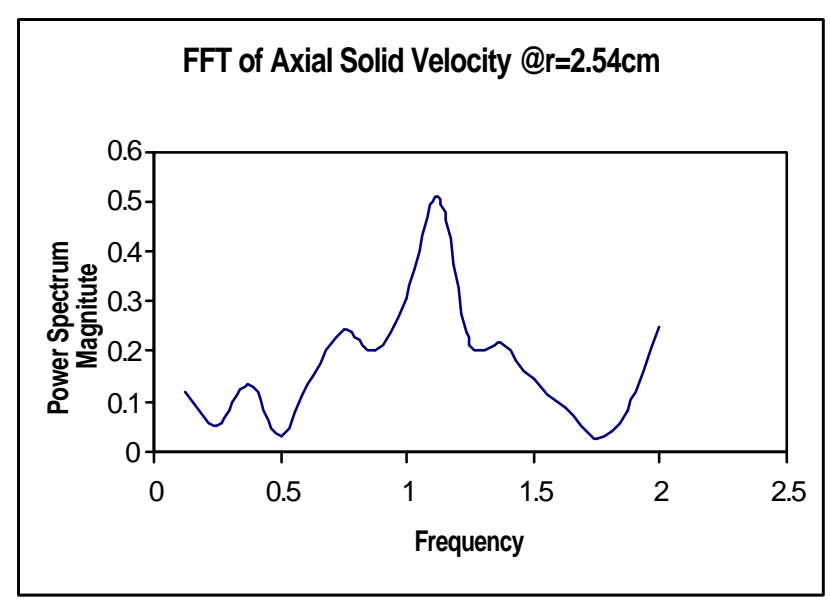

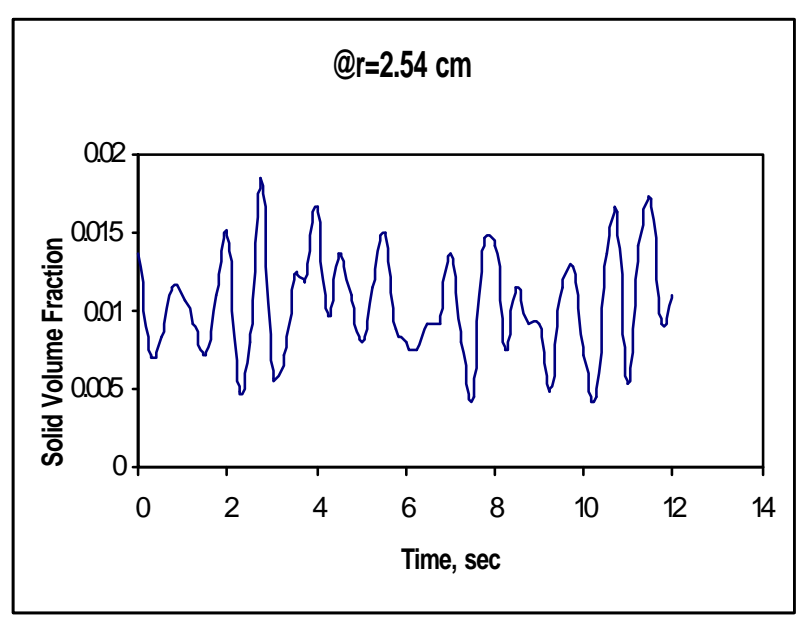

Fig. 14 Time Series of Solid Volume Fraction at $r=2.54 \mathrm{~cm}$

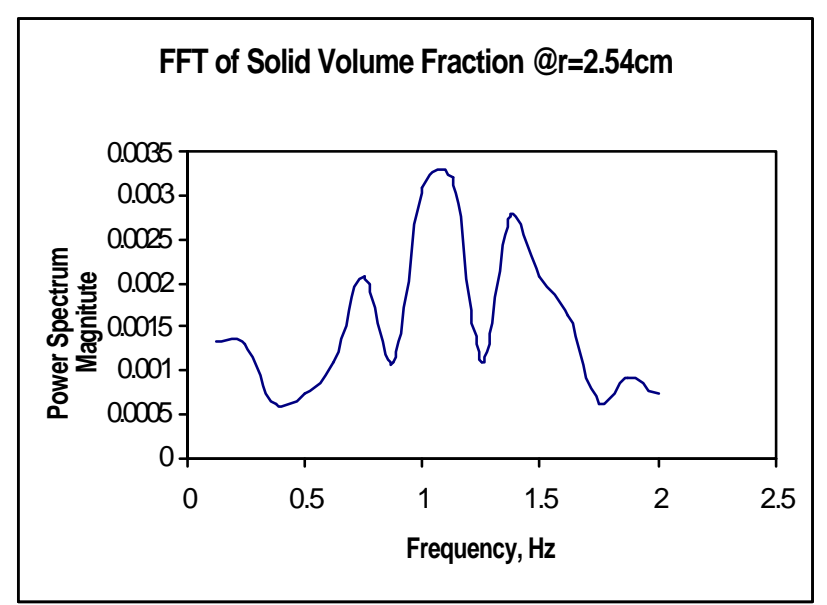

Fig. 15 FFT Analysis of Solid Axial Velocity at $\mathrm{r}=2.54 \mathrm{~cm}$ Fig. 16 FFT Analysis of Solid Volume Fraction at $\mathrm{r}=2.54 \mathrm{~cm}$

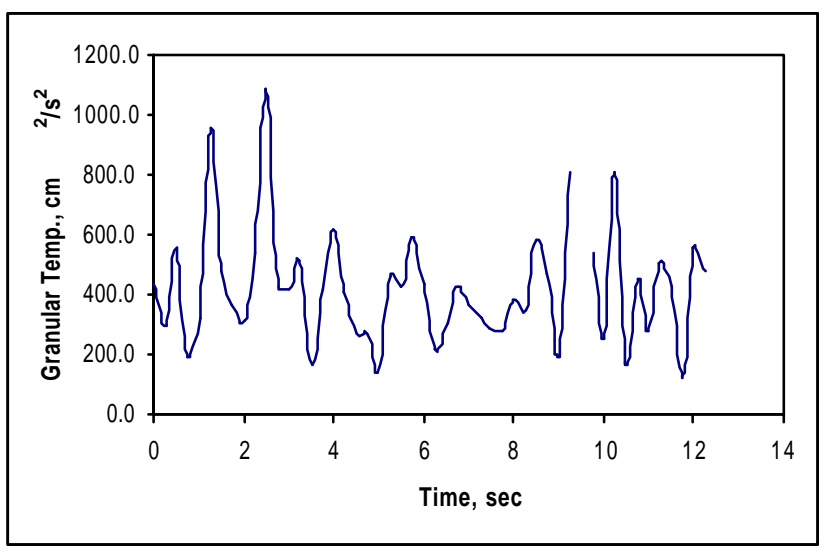

Fig. 17 Time Variation of Granular Temperature at $\mathrm{r}=2.54 \mathrm{~cm}$

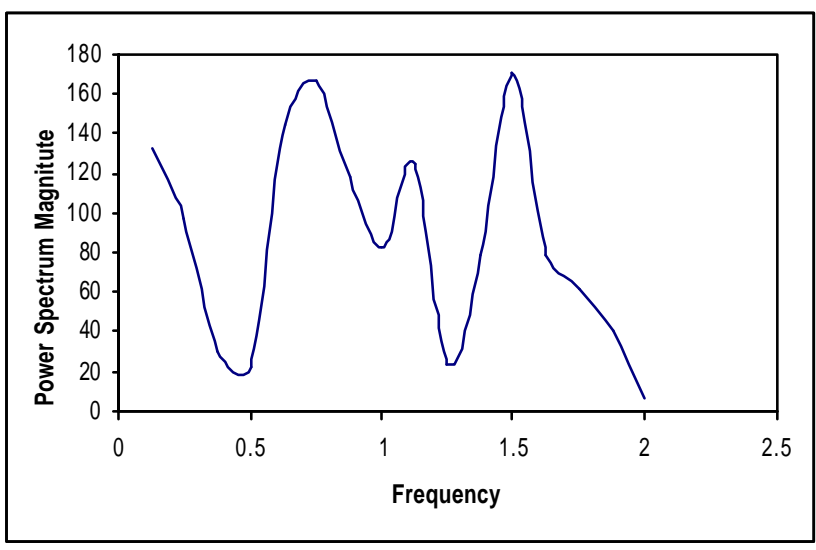

Fig. 18 FFT Analysis of Granular Temperature at $\mathrm{r}=2.54 \mathrm{~cm}$ 


\title{
CFD Models for Slurry Bubble Column Reactors
}

\author{
Isaac K. Gamwo* and John S. Halow \\ National Energy Technology Laboratory \\ United States Department of Energy, P.O. Box 10940 \\ Pittsburgh, PA 15236-0940
}

\section{Dimitri Gidaspow, Reza Mostofi and Diana Matonis}

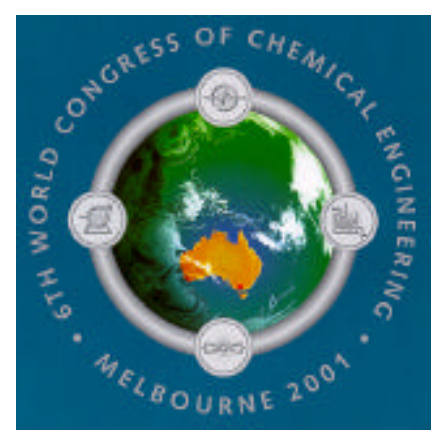

\author{
Dept. of Chemical and Environmental Engineering \\ Illinois Institute of Technology \\ Chicago, IL 60616
}

\begin{abstract}
Two mechanistic Computational Fluid Dynamics (CFD) models are being developed for synthesis of methanol and hydrocarbon liquid fuels using Fischer-Tropsch catalysts in slurry bubble column reactors.

The first model (Wu and Gidaspow, 2000) is based on the kinetic theory of granular flow (Gidaspow, 1994) with a measured restitution coefficient in a slurry bubble column. With a measured restitution coefficient in a slurry bubble column (Mostofi, 2001) the model has predicted Air Products/DOE Laporte reactor's slurry height, gas hold-up and the rate of methanol production. The second hydrodynamic model we have developed consists of two coupled Navier-Stokes equations for gas-liquid-solid flow with a measured slurry viscosity as an input (Matonis, 2000). This model has predicted flow patterns, normal and shear Reynolds stresses in agreement with measurements in a laboratory slurry bubble column.
\end{abstract}

\section{INTRODUCTION}

Slurry bubble column reactors have recently become competitive with traditional tubular fixed-bed reactors for converting syn-gas into liquid fuels due to several advantages, including better temperature control and mass transfer, lower operating and capital costs. The design of these reactors require, among others, precise knowledge of the kinetics, hydrodynamics, and heat as well as mass transfer characteristics. To date, there is no adequate mathematical model available which can predict the performance of slurry bubble column reactors with reasonable accuracy.

\section{HYDRODYNAMIC MODEL}

A transient, isothermal, three-dimensional model for multiphase flow was developed. The hydrodynamic model uses the principle of mass conservation and momentum balance for each phase. See table 1. This approach is similar to that of Soo (1967) for multiphase flow and of Jackson (1985) for fluidization. The equations are 
similar to Bowen's (1976) balance laws for multi-component mixtures. The principle difference is the appearance of the volume fraction of phase " $k$ " denoted by $\mathrm{a}_{k}$. The fluid pressure, $\mathrm{P}$, is in the liquid (continuous) phase.

For gas-solid fluidized beds, Bouillard, et al. (1989) have shown that this set of equations produces essentially the same numerical answers for fluidization as did the earlier conditionally stable model, which has the fluid pressure in both the gas and the solids phases, and is referred to as model B (Gamwo et al., 1995). In this model,. In this model (hydrodynamic model B), the drag and the stress relations were altered to satisfy Archimedes' buoyancy principle and Darcy's Law, as illustrated by Jayaswal (1991). Note in Table 1, no volume fraction is put into the liquid gravity term, while in the gas/solid momentum balance contains the buoyancy term. This is a generalization of model B for gas-solid systems as discussed by Gidaspow (1994) in section 2.4. For the solid phase $\mathrm{P}_{\mathrm{k}}$, consists of the static normal stress and dynamic stress, called the solids pressure, which arises due to the collision of the particles as explained by Gamwo et al. (1999).

This model is unconditionally well-posed, ie, the characteristics are real and distinct for one-dimensional transient flow. It does not require the presence of solid's pressure for stability and well-posedness.

The numerical method is an extension of Harlow and Amsden's (1971) method, which was subsequently used in the K-FIX program (Rivard and Torrey, 1977). The present program was developed from Jayaswal's two-dimensional MICE program (1991); which originated from the K-FIX program (Rivard and Torrey, 1977). To obtain the numerical solution, the non-uniform computational mesh is used in finite-differencing the equations based on the ICE, implicit Eularian method (Rivard,1977; Jayaswal,1991) with appropriate initial and boundary conditions. Stewart and Wedroff (1984) have critically reviewed the ICE algorithm and related staggered mesh conservative schemes. The scalar variables are located at the cell center and the vector variables at the cell boundaries. The momentum equation is solved using a staggered mesh, while the continuity equation is solved using a donor cell method.

Table 1 shows the continuity and the separate phase momentum equations for three-dimensional transient three-phase flow. There are nine nonlinear-coupled partial differential equations for nine dependent variables. The variables to be computed are the

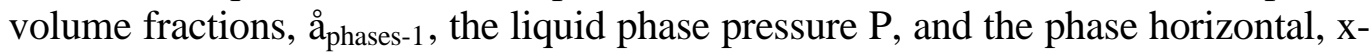
direction, and vertical velocity, $y$-direction components, $u_{\text {phase }}$ and $v_{\text {phase. }}$ The gradient of pressure is in the fluid (continuous) phase only. This leads to an unconditionally wellposed problem, as discussed in detail by Gidaspow (1994) and Lyczkowski, et al. (1978). The details of this model can be found in Matonis (2000) and Matonis et al. (2001).

\section{IIT SLURRY BUBBLE COLUMN}

A rectangular bed was constructed from transparent acrylic (Plexiglas) sheets to facilitate visual observation and video recording of the bed operations such as gas bubbling and coalescence, and the mixing and segregation of solids. The bed height was $213.36 \mathrm{~cm}$ and cross-section was $30.48 \mathrm{~cm}$ by $5.08 \mathrm{~cm}$. A centrifugal pump was connected to the bottom of the bed by a 1.0 -inch $(2.54 \mathrm{~cm})$ diameter stainless steel pipe. 
Gas injection nozzles from an air compressor were connected to the sides of the bed. Liquid was stored in and recycled back to a fifty-five gallon storage tank.

The liquid and gas distributors were located at the bottom of the bed. Two perforated Plexiglas plates with many $0.28 \mathrm{~cm}$ diameter holes distributed the liquid. They were placed at $35.6 \mathrm{~cm}$ and $50.8 \mathrm{~cm}$ above the bottom of the bed, with $0.25 \mathrm{~cm}$ size glass bead particles filled inside. The gas distributor consisted of six staggered porous tubes of $15.24 \mathrm{~cm}$ length and $0.28 \mathrm{~cm}$ diameter. The fine pores of porous tubes had mean diameter of $42 \mathrm{im}$. The porous tubes were placed at the bottom of the bed just below the top liquid distributor plate. Air and water were used as the gas and liquid, respectively, in this experiment. Ballotini (leaded glass beads) with an average diameter of $0.8 \mathrm{~cm}$ and a density of $2.94 \mathrm{~g} / \mathrm{cm}^{3}$ were used as the solids. Figure 1 shows a picture of the experiment for $\mathrm{U}_{\mathrm{g}}=3.36 \mathrm{~cm} / \mathrm{s}$ and $\mathrm{U}_{\mathrm{l}}=2.24 \mathrm{~cm} / \mathrm{s}$. Two distinct bubbles are seen in the middle of the bed.

Figure 2 shows a comparison of two and three dimensional simulation to the experiment. The peaks in velocities correspond to motion of the two bubbles shown in Figure 1. The computed turbulent particle kinetic energy ( $3 / 2$ granular temperature) is shown in Figure 3. The turbulent kinetic energy is nearly constant due to the fact that the particle concentration is nearly uniform in this system.

\section{LaPORTE PILOT PLANT SIMULATION}

$\mathrm{Wu}$ and Gidaspow (2000) developed a model for the production of methanol from syn-gas in an Air/Products/DOE LaPorte slurry bubble column reactor. In their model the viscosity was calculated from the solution of the granular temperature equation. Figure 4 shows the standard Air Products heat exchanger arrangements and two alternate arrangements.

Figures 5 to 7 show the flow patterns and methanol distribution at an instant in the reactors. Figure 8 shows the Methanol production for the three configurations. The model shows an improvement over the present Air Products design.

These simulations illustrate the capability of the model to change the hydrodynamics for an ultimate optimum design. For example in all these three configurations the catalyst diameter was 45 microns. We believe based on measurements of George Cody, formally of Exxon, there exist an optimum particle diameter for maximum turbulence (granular temperature). The catalyst particle diameter can be easily changed in this model to explore its effect on production.

\section{CONCLUDING REMARKS}

A multiphase CFD model was developed. 1- It predicted the experimentally observed flow patterns in a churn turbulent regime. 2- It predicted the turbulent kinetic energy of particles in agreement with measurements. 3- It did not predict the observed bubbles due to coarse grid resolution. 
Table 1- Hydrodynamic Model

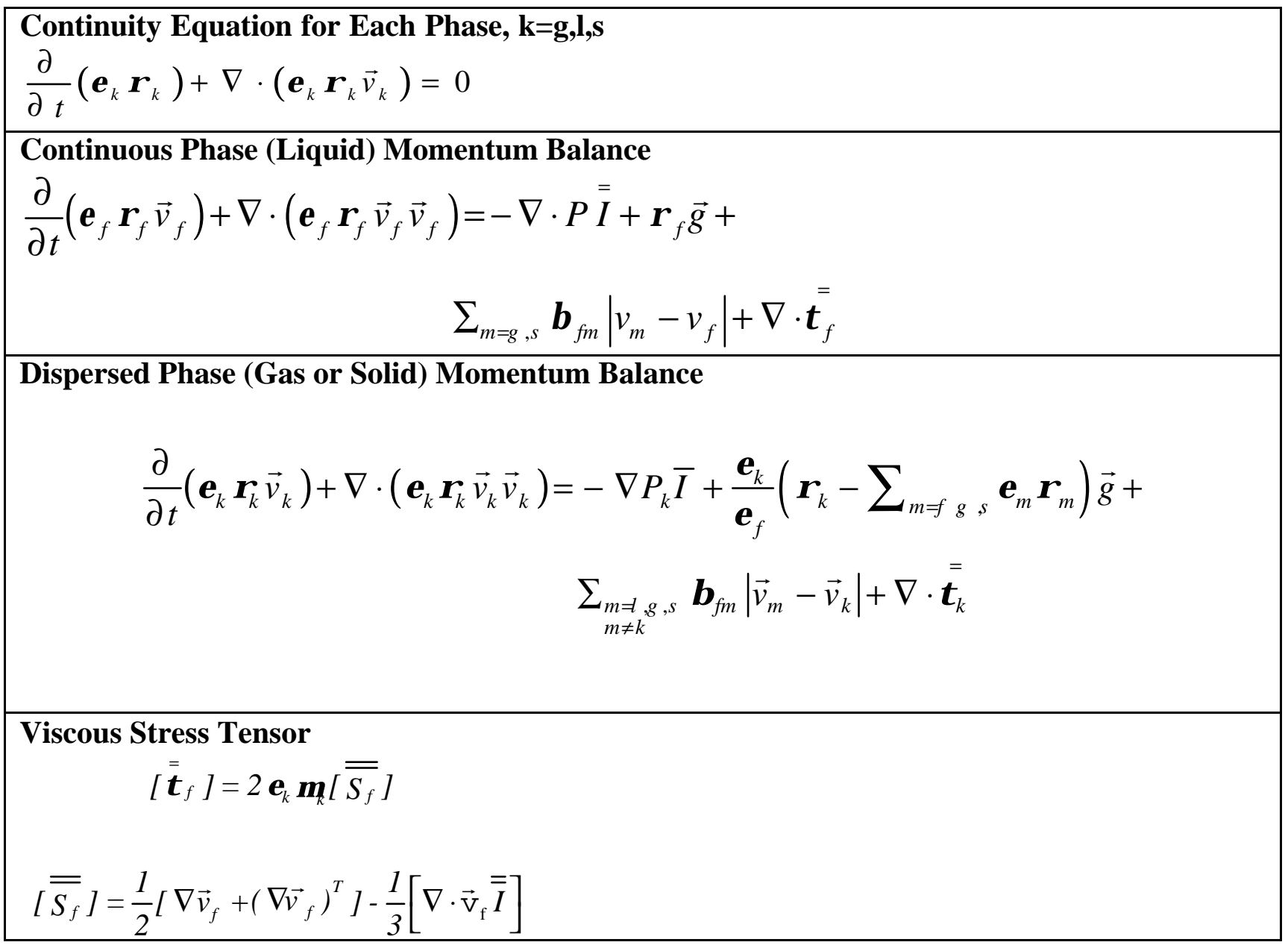

51 


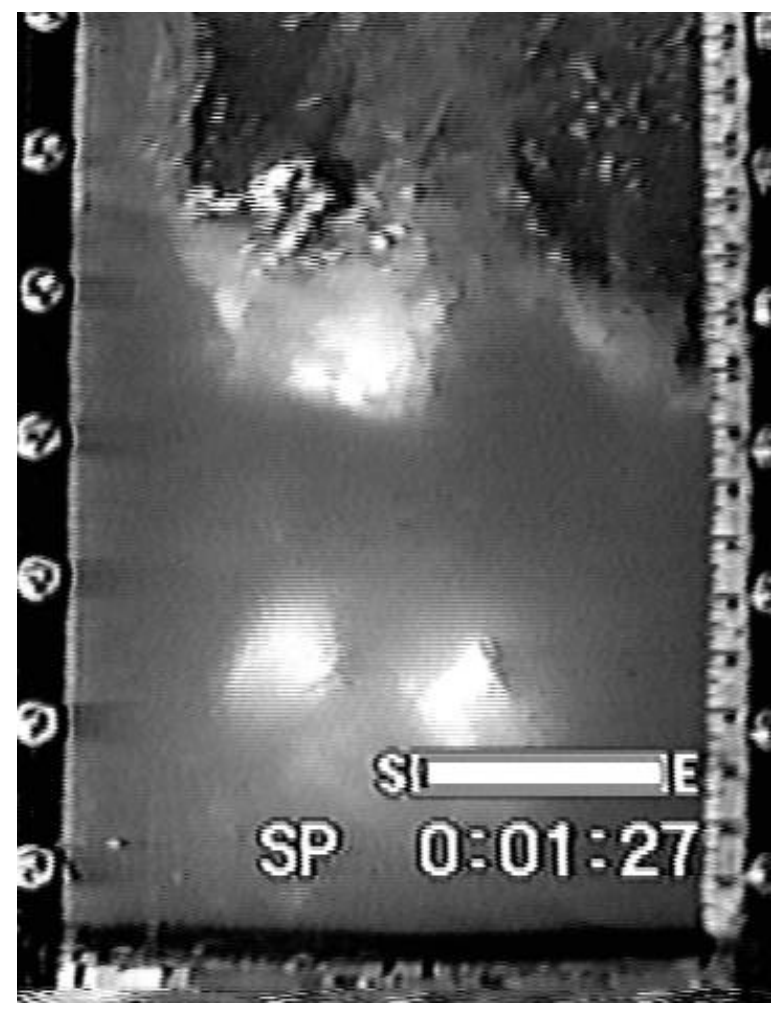

Figure 1- IIT Slurry Bubble Column with Liquid Recirculation 


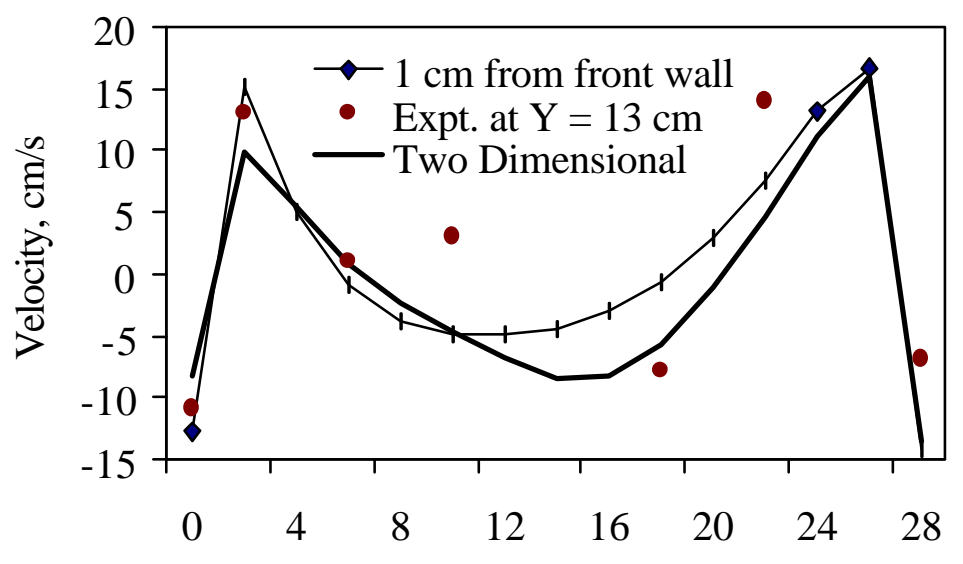

\section{Horizontal Distance from Left Wall, cm}

Figure 2- A Comparison of Two and Three

Dimensional Particle Velocities to PIV Measurements

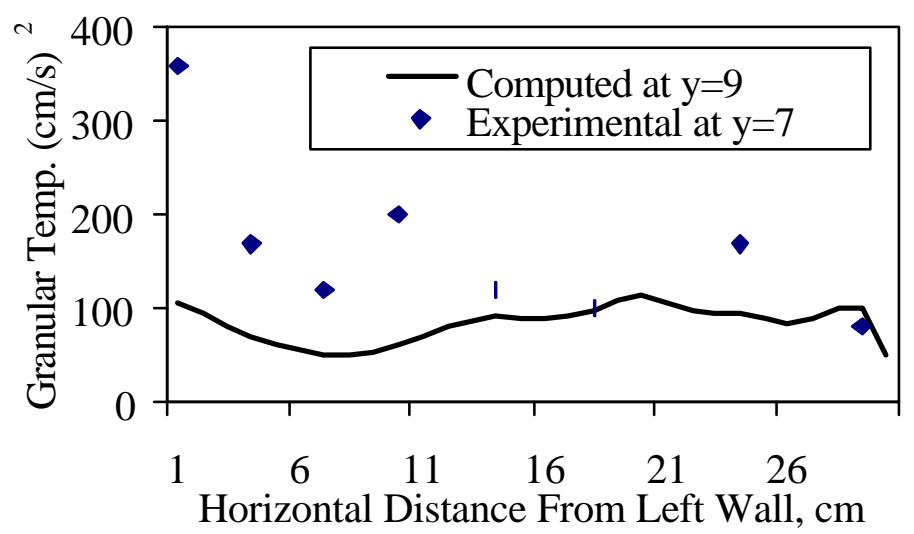

Figure 3- Comparison of Computed and Experimental Granular Temperatures at a bed height of $9 \mathrm{~cm}$ 
Configuration "A"
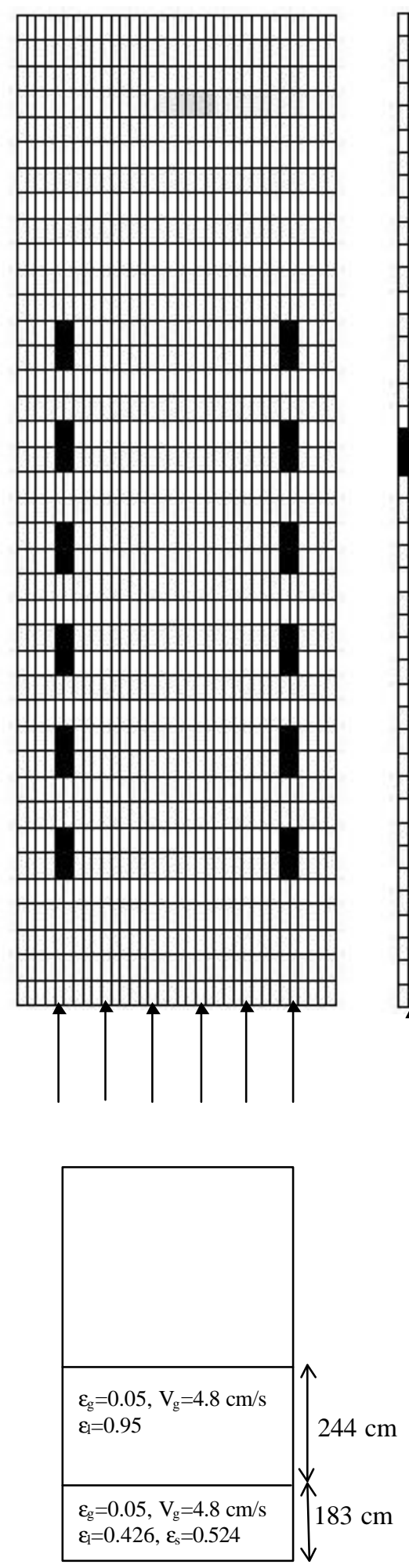

Configuration "B"

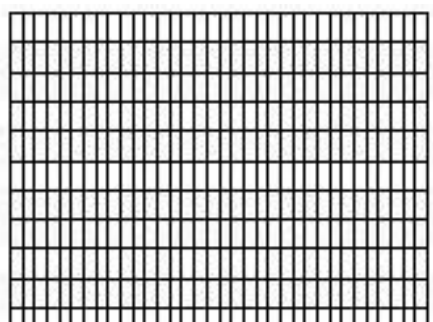

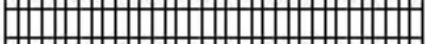

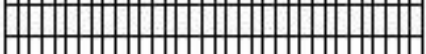

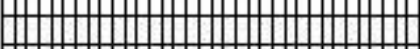

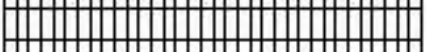

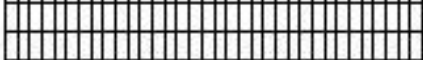

巴田地地地地地

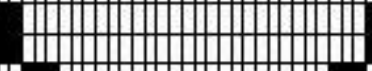

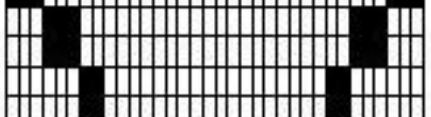

+

囘

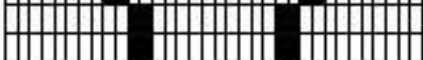

○地

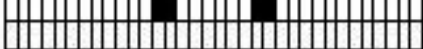



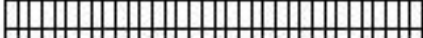

|

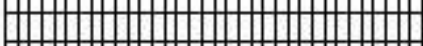

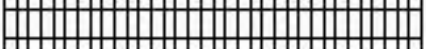

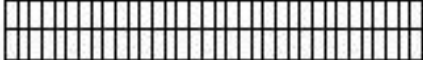

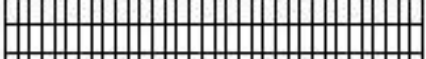

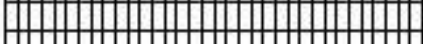

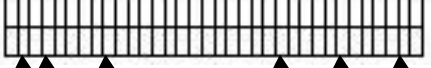

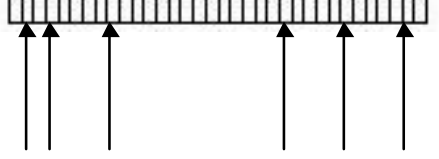

Configuration "C"

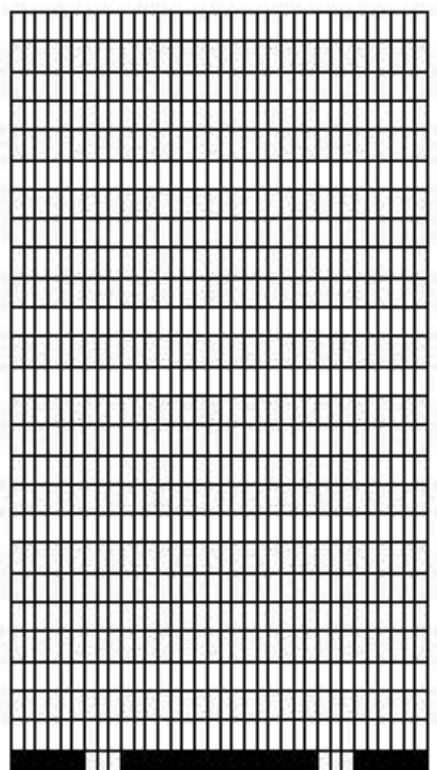

سш

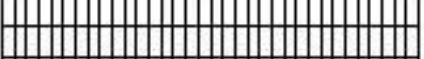

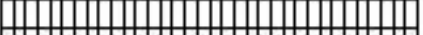

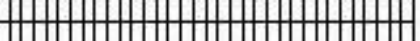
囘

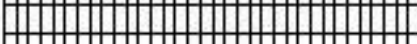

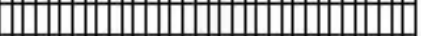

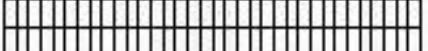

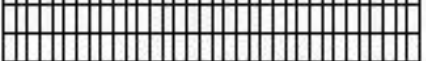

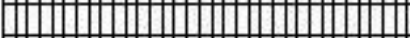
m m m m m m m |

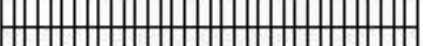
$\left.\right|_{\mid} ^{4} \mid$
Figure 4- Three Different Arrangements of Heat Exchangers in the Slurry Bubble Column reactor and the Initial Conditions 

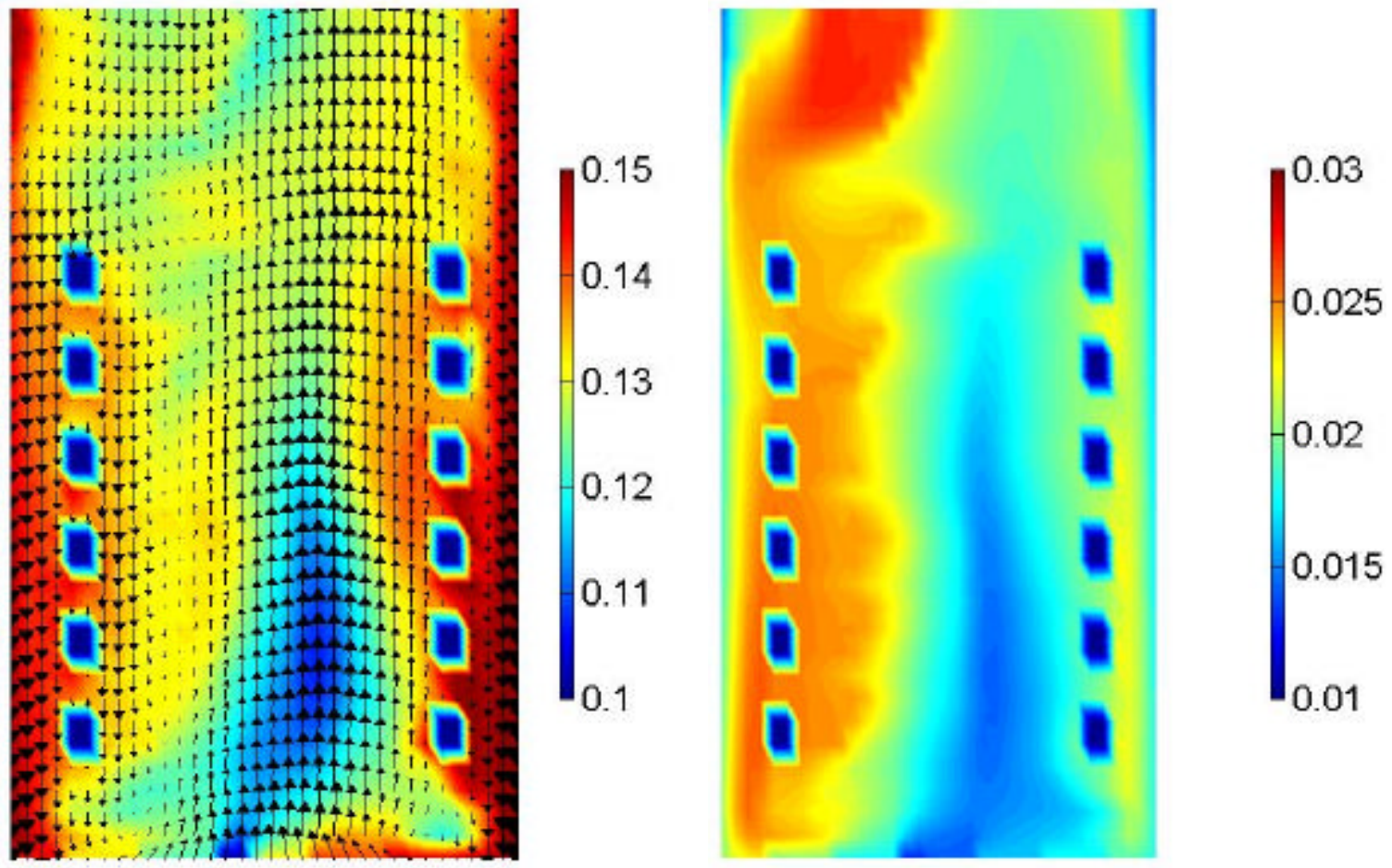

Figure 5- Methanol Mole Fraction in the Gas Phase (right) and Solids Volume Fraction (left) at $\mathrm{t}=70 \mathrm{~s}$ after the start up for heat exchanger set up " $\mathrm{A}$ " 

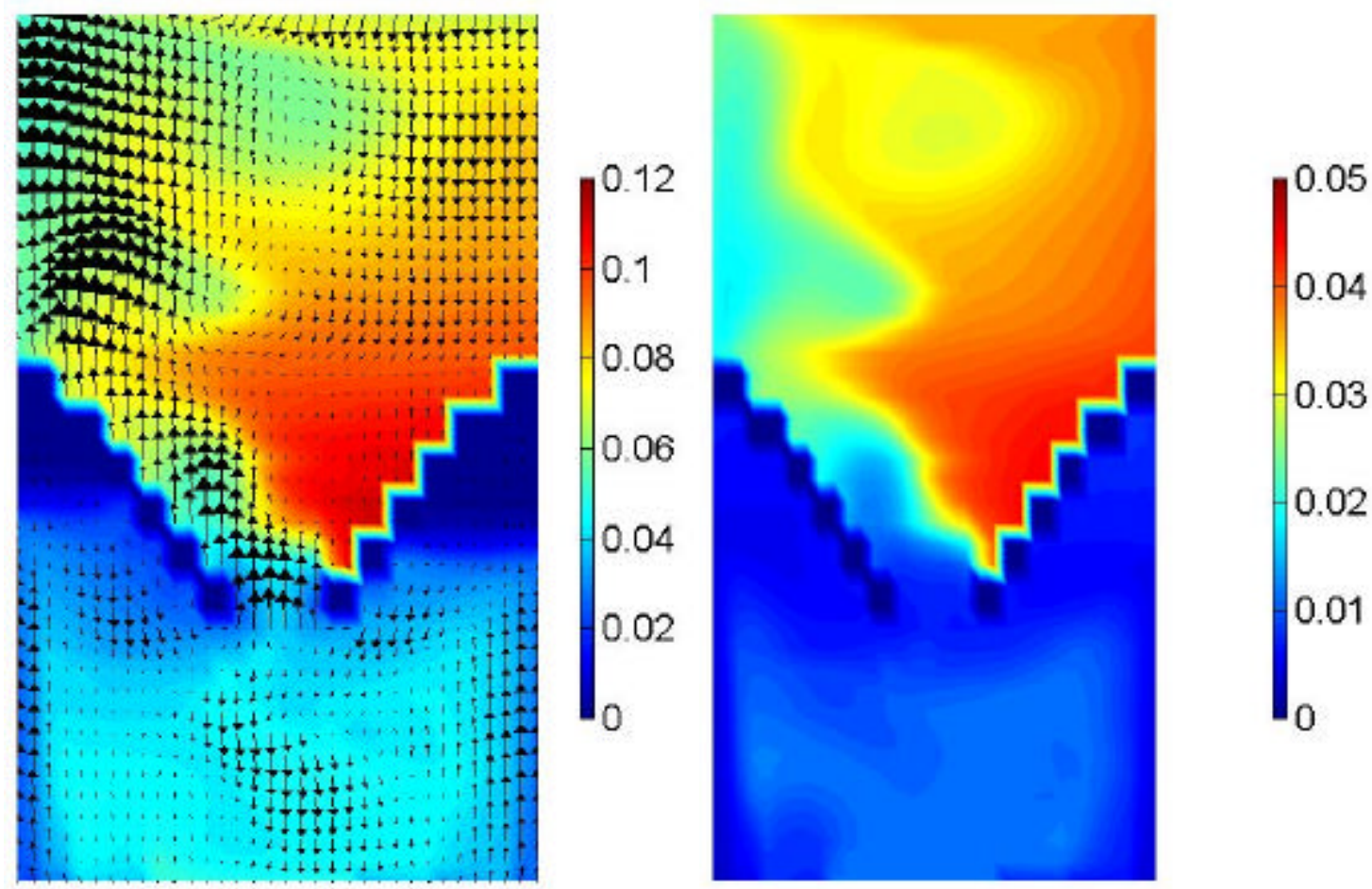

Figure 6- Methanol Mole Fraction in the Gas Phase (right) and Solids Volume Fraction (left) at $\mathrm{t}=70 \mathrm{~s}$ after the start up for heat exchanger set up "B" 


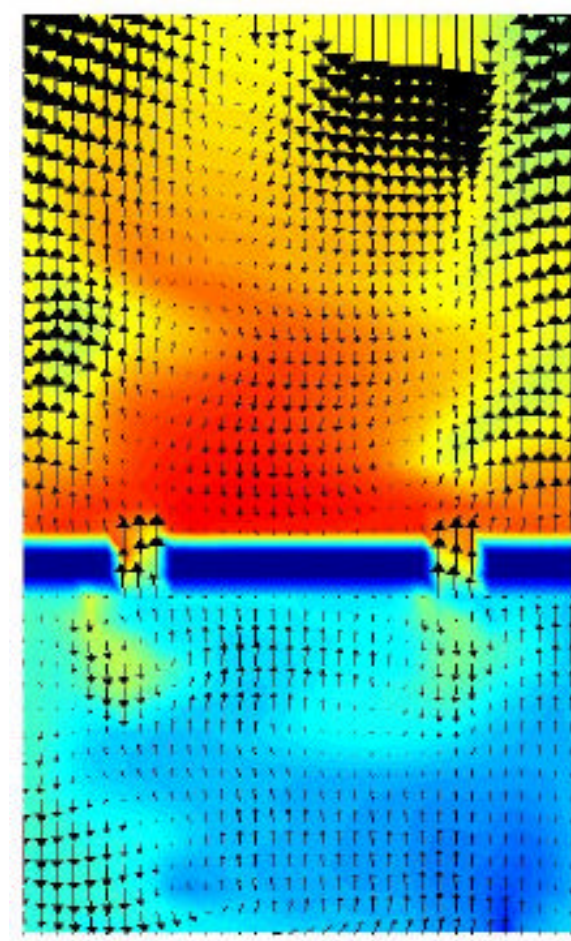

0.12
0.1
0.08
0.06
0.04
0.02
0
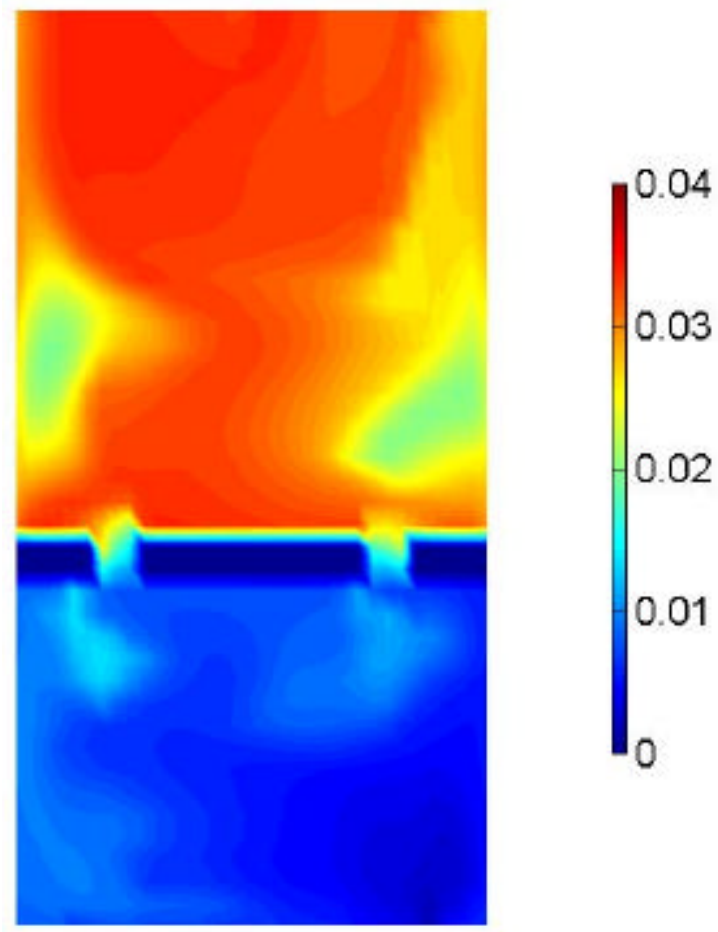

Figure 7- Methanol Mole Fraction in the Gas Phase (right) and Solids Volume Fraction (left) at $\mathrm{t}=70 \mathrm{~s}$ after the start up for heat exchanger set up " $\mathrm{C}$ " 


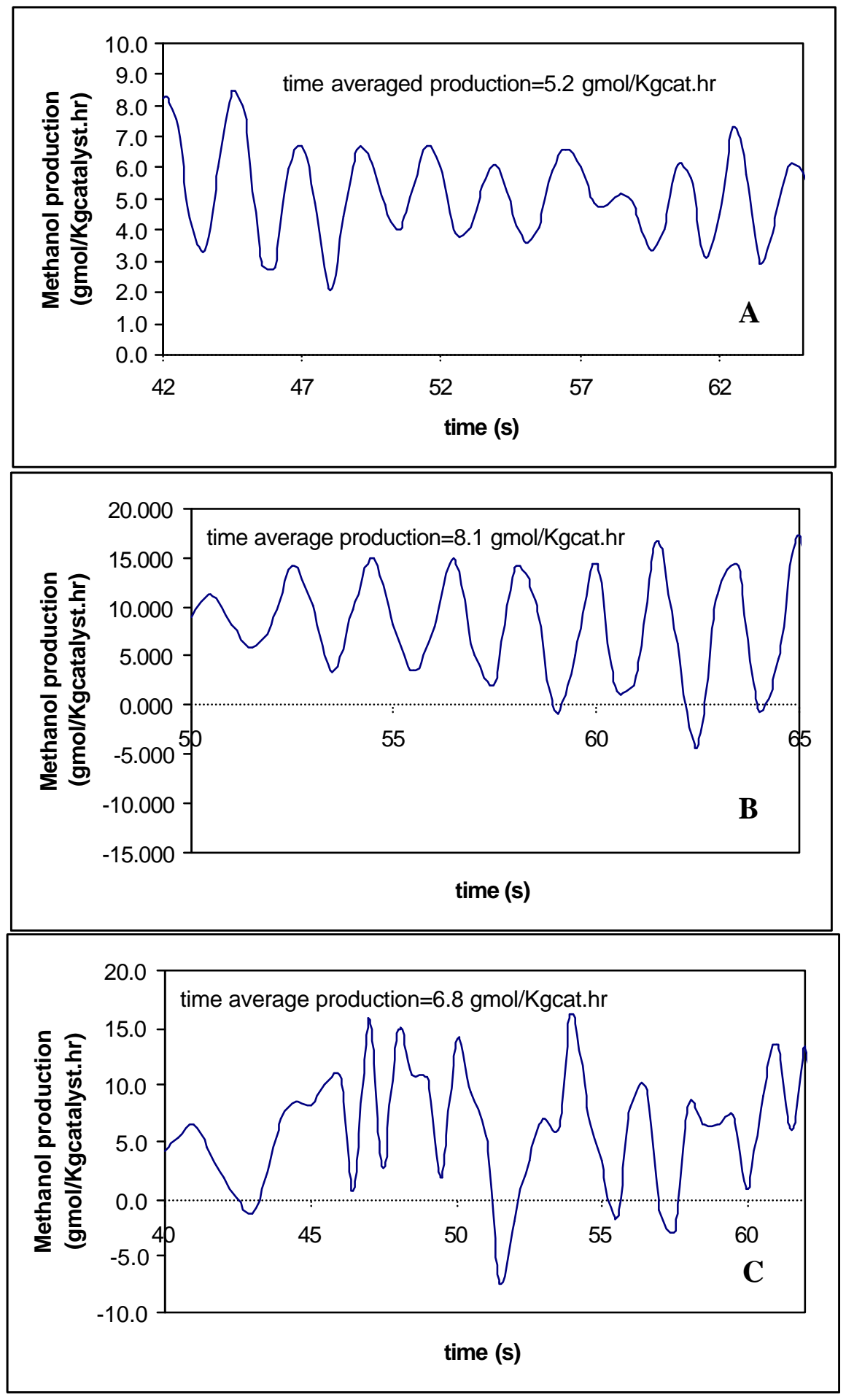

Figure 8- Comparison of the Total Methanol Production for the Three Heat Exchanger Configurations. 


\section{References}

1. Bouillard, J.X. and D. Gidaspow, "Porosity Distribution in a Fluidized Bed with an Immersed Obstacle,” A.I.Ch.E. Journal, 35, 908-922(1989).

2. Bowen, R.M., “Theory of Mixtures," Continuum Pysics, Vol. III, editor A.C. Eringen, Academic Press, New York (1976).

3. Fan, L.-S., "Gas-Liquid-Solid Fluidization Engineering," Butterworths, Boston, Mass. (1989).

4. Gamwo, I. K., Soong, Y., Halow, J. S., 2000, “A Three-Phase Hydrodynamic Model for Slurry Reactors," Recent Advances in the Mechanics of Structured Continua2000, AMD-Vol. 244/MD-Vol. 92, 139-146, edited by M. Massoudi and K. R. Rajagopal, ASME Publications, New York.

5. Gamwo, I. K., Soong, Y. and Lyczkowski, R.W., 1999, "Numerical Simulation and Experimental Validation of Solids Flows in a Bubbling Fluidized Bed," Powder Technology, 103, 117-129.

6. Gamwo, I. K., Soong, Y., Gidaspow, D. and Lyczkowski, R.W., 1995, "ThreeDimensional Hydrodynamic Modeling of a Bubbling Fluidized Bed," Proc. $13^{\text {th }}$ Int. Conf. On Fluidized Bed Combustion, Vol. 1, 297, ASME, New York.

7. Gidaspow, D., "Multiphase Flow and Fluidization: Continuum and Kinetic Theory Descriptions," Academic Press, New York, NY., (1994).

8. Harlow, F.H and A. Amsden, "A Numerical Fluid Dynamics Calculation Method for all Flow Speeds," Jour. of Comp. Physics, 8, 197-213(1971).

9. Jackson, R., "Hydrodynamic Stability of Fluid-Particle Systems," in Fluidization, edited by J.F.Davidson, R. Cliff and D. Harrison, Academic Press, New York, 4772(1985).

10. Jayaswal, U.K., "Hydrodynamics of Multiphase Flows: Seperation, Dissemination and Fluidization," Ph.D. Thesis, Illinois Institute of Technology, Chicago (1991).

11. Lyczkowski, R.W., D. Gidaspow, C.w.Solbrig and E.C. Hughes, "Characteristics and Stability Analysis of Transient One-dimensional Two-Phase Flow Equations and their Finite Difference Aproximations," Nuclear Science and Eng., 66, 378396(1978).

12. Matonis, D., "Hydrodynamic Simulation of Gas-Liquid-Solid Fluidized Bed," PhD Thesis, Illinois Institute of Technology, Chicago (2000).

13. Matonis, D., D. Gidaspow and M. Bahary, "CFD Simulation of Flow and Turbulence in a Slurry Bubble Column ", submitted for publication, (2001).

14. Mostofi, R., PhD Thesis in progress, (August 2001).

15. Rivard, W.C., and M.D. Torrey, "K-FIX: A Computer Program for Transient Twodimensional, Two-Fluid Flow," LA-NUREG-6623, Los Alamos (1977).

16. Soo, S.L., "Fluid Dynamics of Multiphase Systems," Blaisdell Publ. Co., Waltham, Ma., (1967).

17. Stewart, H.B. and B. Wendroff, "Two-Phase Flow: Models and Methods," J. Comput. Phys., 56, 363-409 (1984).

18. Vikings System International, "Design of Slurry Reactor for Indirect Liquefaction Applications," Report to DOE/PETC by A. Prakash and P.B. Bendale, DE-AC2289PC89870. 
19. Wu, Y. and D. Gidaspow, "Hydrodynamic Simulation of Methanol Synthesis in GasLiquid Slurry Bubble Column Reactors," Chem. Eng. Sci., 55, 573-587(2000). 


\title{
Measurement of Thermal Conductivity of FCC Particles
}

\author{
Dimtri Gidaspow Professor, Principal Investigator \\ Lu Huilin, Research Assistant
}

\author{
Department of Chemical and Environmental Engineering \\ Illinois Institute of Technology
}

\section{Introduction}

Various models for heat transfer coefficients in CFB require the knowledge of thermal conductivity of the fluid-particle suspension (Zabrodsky, 1966; Wu, et al, 1987\& 1989; Wu and Gidaspow, 1999). The cluster penetration model (Glicksman, 1988; Basu and Fraser, 1991) shows that the wall to bed heat transfer coefficient is proportional to the square root of the cluster conductivity. The hydrodynamic model of Syamlal and Gidaspow (1985) shows a similar strong dependence on the conductivity. Kuipers, et al (1992) have confirmed and extended the computations.

A heat transfer module was inserted into the two-story CFB at IIT. From time average radial temperature distributions at two axial positions, the thermal conductivity of FCC particles was calculated using an energy balance for the mixture. This procedure was very similar to the method of computation of FCC viscosity from measurement of radial velocity profiles (Miller and Gidaspow, 1992). Similarly to the viscosity the conductivity is a function of the volume fraction of FCC particles. At 5\% particles the kinematic viscosity is $0.15 \mathrm{~cm}^{2} / \mathrm{s}$, while the thermal diffusivity is only $0.036 \mathrm{~cm}^{2} / \mathrm{s}$. Hence the Prandtl number has a reasonable value of 4.2.

\section{Experimental Equipment: Circulating fluidized bed}

The circulating fluidized bed (CFB) test unit shown in Figure 1 was used. Air enters the CFB through the bottom U section with FCC catalyst particles of a diameter of $75 \mu \mathrm{m}$, density of $1654 \mathrm{~kg} / \mathrm{m}^{3}$ and specific heat of $2.6 \mathrm{KJ} / \mathrm{Kg} . \mathrm{K}$. The set-up is that used by Miller and Gidaspow (1992). A section of the acrylic pipe was replaced with a heat transfer module.

\section{Heat Transfer Module}

Two electrical heaters with wire coils, internal and compensator heater, were used as the heat source, shown in Figure 2. The wire coils were arranged uniformly so that heat was liberated quite uniformly along the height. The diameter of wire coil is $1.0 \mathrm{~mm}$ and $0.4 \mathrm{~mm}$ for internal and compensator heater, respectively. The heating length of 
heater is $1.016 \mathrm{~m}$. Both heaters are separated by a polyurethane foam strip. Fiberglass was used to separate the wire coils from both the riser pipe and insulation. Inside of the insulation between the two heaters, two thermocouples (type T) were installed in order to control the energy added to the compensator. The signals of these thermocouples are fed to temperature controllers (Ysi, Model 72) which maintain a temperature difference equal to zero, avoiding heat loss. Power fed to the internal electrical heater was controlled by a powerstat, which can be adjusted to the required voltage.

Measurement of particle temperature is difficult because it is not possible to attach a thermocouple without impairing the mobility of the particles. Usually, temperatures in fluidized beds are measured with thermocouples in direct or indirect contact with particles. Protected thermocouples at low temperature conditions are not only inaccurate, but also have a low response characteristic. Unshielded thermocouples measure some mean temperature lying between the gas temperature and the particle temperature.

Temperatures were measured by two unshielded copper-constant thermocouples each separated $0.45 \mathrm{~m}$. Moving the thermocouples back and forward along the radial coordinate, a radial temperature profile can be determined. Transient temperature data using these thermocouples were collected. The $\mathrm{mV}$ signals from the thermocouples passed through an amplifier and then to an analog-digital converter (11 channels) made by Interactive Strutures Inc. The digital signals were stored by a computer.

Experiments were done under steady state conditions. Results of a calibration test show that the steady state was reached 1.5-2.0 hours after switching on the heater. For a steady state the two thermocouples across the insulation had to give equal temperature.

\section{Extraction Probe}

Flux profiles in the CFB were measured with the extraction probe shown in Figure 3. The probe consisted of a brass tube that has an outside diameter of $0.635 \mathrm{~cm}$ and a $0.4724 \mathrm{~cm}$ inside diameter. The tube is bent at a 90-degree angle to align its opening parallel to the flowing gas-solids mixture. After extraction by the probe, the gas/solids mixture is separated by a filter. The filter is equipped with a removable solids collection chamber that is fabricated of clear PVC for visual observation. Air leaving the filter flows through a fine secondary filter to protect the downstream rotameter and a vacuum pump. The flow rate of air exiting the extraction probe was adjusted by a globe valve that is located downstream of the rotameter.

At any particular position within the CFB, the time-averaged solid flux consisted of an upward and downward component. The magnitude of the upward flux minus the magnitude of the downward flux is equal to the net upward flux which should be equal to external solids flux. The upward and downward fluxes are measured with the inlet of the extraction probe pointing in the upstream and downstream directions, respectively.

Because the CFB operates in an oscillatory state, all flux measurements were timeaveraged over periods that ranged from 1,5 to 8 minutes. Flux components were calculated by dividing the weight of solids collected in the filtration chamber by the area of the tip of the probe and sample time.

Van Breugel et al. (1969) suggested in their study that the extraction probe should be operated under isokinetic conditions, that means the velocity in the probe nozzle 
should be equal to that of the surrounding stream. However, the CFB operates with a rapid fluctuating velocity, making it very difficult to operate the probe isokinetically. Similar to Gajdos and Bierl (1978), we have found that the flux withdrawn by the extraction probe does not vary significantly when the probe gas extraction velocity is altered within the range of gas velocity anticipated at the local positions in the CFB. This is probably due to the overwhelming size of the momentum of the solids compared to that of the gas. The magnitude of both flux components at the riser wall with varying extraction velocity in the range of 2.0-12.0 m/s is shown in Figure 4. This behavior shows that the downward minus upward flux is approximately constant at the riser wall. Downward flux at the center of the riser was negligible in comparison to the corresponding upward flux for all measured conditions.

Near the riser wall it is necessary to measure both the downward and upward fluxes to obtain the net flux. Flow-restrictive plugging, however, would sometimes occur when the extracting velocity of the probe operated within the range of low gas velocity. There is little difference in the magnitude of the upward and downward fluxes. Therefore, in this investigation the net flux has been taken to be the difference between the two components of the flux measured at the same extracting velocity. The error analysis indicated that the main error for the thermal conductivity and heat transfer coefficient was caused by the error of measuring the solid mass flux. To compensate for the experimental error, the readings were averaged over two extracting velocities. Under such conditions the error between net upward flux and the external flux was estimated to be less than $18.2 \%$.

\section{Model}

The steady state differential equation for equal solid and gas temperature can be derived for the differential element shown in Figure 5. It is:

$$
\left(W_{s} C_{p}+G_{g} C_{g}\right) \frac{\partial T}{\partial z}=K_{r}\left(\frac{\partial^{2} T}{\partial r^{2}}+\frac{1}{r} \frac{\partial T}{\partial r}\right)
$$

The assumption of symmetry gives:

$$
\left.\frac{\partial T(Z, 0)}{\partial r}\right|_{r=0}=0
$$

The following equation for the mean thermal conductivity is obtained after integrating Eq. (1):

$$
\int_{0}^{R}\left(W_{s} C_{p}+G_{g} C_{g}\right) \frac{\partial T}{\partial z} r d r=\left.R K_{r} \frac{\partial T}{\partial r}\right|_{r=R}
$$

The temperature profiles and solid mass flux profiles are obtained by approximating the data with a least-squares polynomial that visually best fits the profiles. The polynomial ranged in order from 5th to 8th degree.

The mean overall heat transfer coefficient, h, was obtained by taking an overall heat balance across the whole riser. If $\mathrm{Q}$ is the net heat flux, the heat transfer coefficient can be estimated by: 
$Q=h A \Delta T$

(4)

where $\mathrm{A}$ is the heat transfer surface based on the internal diameter of the riser, and $\Delta \mathrm{T}$ is the logarithmic temperature difference.

\section{Experimental Data: Radial Temperature Profile}

Typical local instantaneous temperatures are shown in Figure 6. In general, variation of instantaneous temperature indicated the degree of mixing and exchange between particles, particle and gas. It is seen that the degree of such mixing near the wall is more intense than that in the core region. The flow characteristic is approaching plug flow in the core region.

Radial temperature profiles experimentally obtained in present test are shown in

Figure 7. In general, the radial temperature gradient is near straight line in the core region and very steep near the wall of the riser.

\section{Solid Flux Profile}

Solid flux profiles were obtained using the extraction probe. The profiles are quasi-parabolic with an upward-moving mass flux at the center and downward flux at the wall. In Figure 8, the radial mass flux is plotted at a constant superficial velocity. The radial mass flux at the center of the riser increases with increasing feed flux. At the wall the solids are flowing downward. The magnitude of the flux increases with increasing values of the feed flux. The core is defined as the region in the center of the riser in which the net solid mass flux is moving upward. It is seen that the radius of the core decreases with increasing values of mass flux.

The influence of the superficial velocity on the radial flux profiles is shown in Figure 9. The core radius increases with increasing superficial velocity. The solid mass flux at the centerline decreased with increasing superficial velocity. The magnitude of the solids mass flux at the wall flowing downward increases as the superficial velocity decreases. The profiles of radial mass flux are near parabolic.

\section{Thermal Conductivity}

The plot of the effective thermal conductivity versus the superficial velocity is presented in Figure 10. The effective thermal conductivity increases slightly with increasing superficial velocity. The influence of the solid mass flux on the effective thermal conductivity is presented in Figure 11. The value of the effective thermal conductivity increases with increasing solid mass flux. This is due to the particle concentration near the wall. It may be seen that the individual values of the thermal conductivity evaluated from each radial temperature profile become independent of axial position in this present system, when the axial distance is large. Therefore, it may be reasonable to obtain the thermal conductivity from the temperature profiles under these testing conditions.

The effective thermal conductivity versus cross sectional averaged solid volume fraction is shown in Figure 12. It is seen that the effective thermal conductivity increases 
with increasing solid volume fraction in the dilute region of the circulating fluidized beds. The least squares fitted equation is:

$$
K_{r}=[5.9427+366.218(1-\varepsilon)] 10^{-2}
$$

Let us assume that

$$
K_{r}=K_{g, o}+K_{s}
$$

where $\mathrm{K}_{\mathrm{go}}$ is the gas thermal conductivity.

Figure 13 shows the variation of the effective particle thermal conductivity $\mathrm{K}_{\mathrm{s}}$ with the solid volume fraction. It is seen that the effective particle thermal conductivity increases with increasing solid volume fraction. The expression obtained by least squares fitting of data is:

$$
K_{s}=[3.1995+366.098(1-\varepsilon)] 10^{-2}
$$

\section{Heat Transfer Coefficient}

Figure 14 shows the variation of heat transfer coefficient with superficial velocity. Within the range of superficial velocity tested, the data shows that the heat transfer coefficient increases slightly with increasing superficial velocity. There is a very substantial augmentation of the heat transfer coefficient with superficial velocity. According to Grace (Grace, 1986), the independence of heat transfer of the superficial velocity is due to the small contribution of the gas convective component to the heat transfer coefficient. The variation of the gas convetive component due to a change in gas velocity for a flow of gas alone is indeed small compared to the measured variations at higher solid mass flux. Figure 15 shows the influence of solid mass flux on the heat transfer coefficient. It is evident from this figure that heat transfer coefficient is a strong function of the solid mass flux.

The plot of the heat transfer coefficient versus bulk density is shown in Figure 16. It is seen that the heat transfer coefficient increases with increasing density in the dilute region of the circulating fluidized bed. The least-squares correlation is as follows: $h=87.466+1.5038 \rho_{b}$

where $\rho_{b}$ is bulk density of the riser.

\section{Error Analysis}

The expected error in the measured values of the effective thermal conductivity can be expressed as follows:

$$
\varepsilon_{K_{r}}=\left|\frac{\Delta K_{r}}{K_{r}}\right|^{2}=\left|\frac{\Delta W_{s}}{W_{s}}\right|^{2}+\left|\frac{\Delta G_{g}}{G_{g}}\right|^{2}+\left|\frac{\Delta T}{T}\right|^{2}+\left|\frac{\Delta r}{R}\right|^{2}+\left|\frac{\Delta Z}{L}\right|^{2}+\text { End Effects }
$$

The accuracy of measured solid mass flux $\mathrm{W}_{\mathrm{s}}$ is usually within $\pm 18.2 \%$ using the extraction probe. The accuracy of gas mass flux, $\mathrm{G}_{\mathrm{g}}$ is estimated to be $\pm 4.5 \%$, metered 
by rotameters. The temperature measurement using the copper-constant thermocouple is within $\pm 1.0 \%$, while the accuracy of measuring $\mathrm{r}$ and $\mathrm{Z}$ are about $\pm 1.33 \%$ and $\pm 0.2 \%$, respectively. The end effects cannot be predicated accurately. The measured value of the thermal conductivity is then within $\pm 19 \%$. The main error is due to the measured solid mass flux.

The expected error for heat transfer coefficient can be written as follows:

$\varepsilon_{h}=\left|\frac{\Delta h}{h}\right|^{2}=\left|\frac{\Delta Q}{Q}\right|^{2}+\left|\frac{\Delta W_{s}}{W_{s}}\right|^{2}+\left|\frac{\Delta G_{g}}{G_{g}}\right|^{2}+\left|\frac{\Delta T}{T}\right|^{2}+\left|\frac{\Delta r}{R}\right|^{2}+\left|\frac{\Delta Z}{L}\right|^{2}+$ End Effects

The accuracy of measuring the input power is within $\pm 3.5 \%$. The measured value of the heat transfer coefficient is then $\pm 19.4 \%$.

\section{Nomenclature}

A Heat transfer surface

$\mathrm{C}_{\mathrm{p}} \quad$ Particle specific heat

$\mathrm{m}^{2}$

$\mathrm{C}_{\mathrm{g}} \quad$ Gas specific heat

$\mathrm{H} \quad$ Heat transfer coefficient

$\mathrm{G}_{\mathrm{g}} \quad$ Gas mass flux

$\mathrm{K}_{\mathrm{r}} \quad$ Effective thermal conductivity

$\mathrm{K}_{\mathrm{s}} \quad$ Effective particle thermal conductivity

$\mathrm{K}_{\mathrm{g}} \quad$ Gas thermal conductivity

$\mathrm{K}_{\mathrm{g}, \mathrm{o}} \quad$ Gas microscopic thermal conductivity

$\mathrm{K}_{\mathrm{s}, \mathrm{o}} \quad$ Solid microscopic thermal conductivity

$\mathrm{P} \quad$ Pressure

Q Net heat flux

$\mathrm{R} \quad$ Radial coordinate

$\mathrm{R} \quad$ Radius of riser

$\mathrm{T} \quad$ Gas/particle mixture temperature

$\mathrm{T}_{\mathrm{g}} \quad$ Gas temperature

$\mathrm{T}_{\mathrm{S}} \quad$ Particle temperature

$\mathrm{T}_{\mathrm{r}=\mathrm{R}} \quad$ Wall temperature

$\mathrm{T}_{\mathrm{r}=0} \quad$ Centerline temperature

$\mathrm{U}_{\mathrm{g}} \quad$ Gas velocity

$\mathrm{W}_{\mathrm{s}} \quad$ Solid mass flux

$\mathrm{Z} \quad$ Axial coordinate

$\mathrm{J} / \mathrm{Kg} . \mathrm{K}$

$\mathrm{J} / \mathrm{Kg} . \mathrm{K}$

$\mathrm{W} / \mathrm{m}^{2} \cdot \mathrm{K}$

$\mathrm{Kg} / \mathrm{m}^{2} . \mathrm{s}$

$\mathrm{W} / \mathrm{m} . \mathrm{K}$

$\mathrm{W} / \mathrm{m} . \mathrm{K}$

$\mathrm{W} / \mathrm{m} . \mathrm{K}$

$\mathrm{W} / \mathrm{m} . \mathrm{K}$

$\mathrm{W} / \mathrm{m} . \mathrm{K}$

$\mathrm{N} / \mathrm{m}^{2}$

$\mathrm{W}$

$---$

$\mathrm{m}$

$\mathrm{K}$

$\mathrm{K}$

$\mathrm{K}$

$\mathrm{K}$

$\mathrm{K}$

$\mathrm{m} / \mathrm{s}$

$\mathrm{Kg} / \mathrm{m}^{2} . \mathrm{s}$

Greek letters:

$\rho_{\mathrm{g}} \quad$ Gas density $\mathrm{Kg} / \mathrm{m}^{3}$

$\rho_{\mathrm{s}} \quad$ Solid density $\mathrm{Kg} / \mathrm{m}^{3}$

$\rho_{\mathrm{b}} \quad$ Bulk density $\mathrm{Kg} / \mathrm{m}^{3}$

$\varepsilon \quad$ Porosity 


\section{References}

Basu P. and K. Nag, An investigation into heat transfer in circulating fluidized beds. Int. J. Heat Mass Transfer, No. 30, pp. 2399-2409, 1987.

Basu P. and S.C. Fraser, Circulating Fluidized Bed Boilers: Design and Applications, Butterworth-Heinemann Inc., 1991.

Botterill J. S. M. and A. O. O. Denloye, A theoretical model of heat transfer to a packed or quiescent fluidized bed, Chemical Engineering Science, No. 4, pp. 509-515, 1978.

Ebert T. A., L. R. Glicksman and M. Lints, Determination of particles and gas convective heat transfer components in a circulating fluidized bed, Chemical Engineering Science, No. 12, pp. 179-188, 1993.

Ernst R. G. E. and R. J. Goldstein, Measurements in heat transfer, McGraw-Hill Book Company, 1976.

Gabor J. D., B. E. Stangeland and W. J. Mecham, Lateral transport in a fluidized-packed bed: part p: heat transfer, AIChE J., No. 1, pp. 130-132, 1965.

Gajdos J. L. and T. W. Bierl, Studies in support of recirculating bed reactors for the processing of coal, Annual report, Prepared U. S. Dept. of Energy, FE-2449-8 (Sept.), 1978.

Gidaspow, D., Multiphase flow and fluidization: continuum and kinetic theory descriptions, Academic Press Inc. 1994.

Glicksman L.R., Circulating Fluidized Beds Heat Transfer, in Circulating Fluidized Bed Technology II, Ed. By P. Basu and J.F. Large, Pergamon Press, 1988.

Grace J. R., Heat transfer in circulating fluidized beds, First Int. Conf. on Circluating Fluidized Beds, Halifax, 1986.

Kuipers J.A.M., W. Prins and W.P.M. van Swaaij, Numerical Calculation of Wall-to-Bed Heat Transfer Coefficients in Gas-Fluidized Beds, AIChE J., No. 7, 1992.

Miller A. and D. Gidaspow, Dense, vertical gas-solids flow in a pipe, AIChE J., No. 11, pp. 1801-1815, 1992.

Syamlal M. and D. Gidaspow, Hydrodynamics of fluidization: prediction of wall to bed heat transfer coefficients, AIChE. J., No. 1 pp. 127-135, 1985.

Van Breugel J. W., J. J. M. Stein and R. J. Devries, Isokinetic sampling in a dense gassolids stream, Joint Symp. on Fluid Mech, and Measurements in Two Phase Systems.

The Institution of Mechanical Engineers, Leeds (Sept.), 1969.

Wu Y. and D. Gidaspow 1999, Hydrodynamic simulation of methanol synthesis in gasliquid slurry bubble column reactors, to appear in Chem. Eng. Science.

Wu R. L., C. J. LIM, J. Chouki and J. R.Grace, Heat transfer from a circulating fluidized bed to membrane water wall surfaces, AIChE J., No. 33, pp. 11, 1987.

Wu R. L., J. R. Grace, C. J. Lim and C. M. H. Brereton, Suspension-to-surface heat transfer in circulating fluidized bed combustor, AIChE J., No. 35, pp. 1685-1691, 1989. Zabrodsky S. S., Hydrodynamics and heat transfer in fluidized beds, The M.I.T. press, 1966. 
Table 1

Experimental Conditions

\begin{tabular}{||c|c|c|c||}
\hline Run & Velocity $(\mathrm{m} / \mathrm{s})$ & Flux $\left(\mathrm{Kg} / \mathrm{m}^{2} . \mathrm{s}\right)$ & Voltage $(\mathrm{V})$ \\
\hline No. 1 & 3.545 & 17.48 & 65.04 \\
\hline No. 2 & 3.529 & 23.03 & 55.46 \\
\hline No. 3 & 3.553 & 32.09 & 80.92 \\
\hline No. 4 & 2.365 & 17.36 & 67.85 \\
\hline No. 5 & 3.020 & 24.43 & 72.88 \\
\hline No. 6 & 2.982 & 26.37 & 72.28 \\
\hline No. 7 & 2.982 & 17.26 & 64.27 \\
\hline No. 8 & 3.580 & 16.85 & 45.45 \\
\hline No. 9 & 3.562 & 26.80 & 75.45 \\
\hline No. 10 & 3.010 & 27.12 & 65.45 \\
\hline No. 11 & 2.419 & 23.90 & 75.45 \\
\hline No. 12 & 2.408 & 10.39 & 67.78 \\
\hline No. 13 & 3.607 & 16.75 & 67.78 \\
\hline No. 14 & 387 & 11.67 & 46.55 \\
\hline
\end{tabular}

\begin{tabular}{||c|c|c|c|}
\hline Run & $\begin{array}{c}\text { External } \\
\text { solid Flux }\end{array}$ & $\begin{array}{c}\text { Net solid } \\
\text { Flux }\end{array}$ & Error(\%) \\
\hline No.1 & 17.48 & 16.299 & 6.76 \\
\hline No. 2 & 23.03 & 26.586 & -15.44 \\
\hline No. 3 & 32.09 & 35.516 & 10.68 \\
\hline No. 4 & 17.36 & 20.518 & -18.19 \\
\hline No. 5 & 24.43 & 27.015 & 3.70 \\
\hline No. 6 & 26.37 & 28.227 & -7.04 \\
\hline No. 7 & 17.26 & 14.272 & 17.31 \\
\hline No. 8 & 16.85 & 17.557 & -19 \\
\hline No. 9 & 26.80 & 22.896 & 14.57 \\
\hline No. 10 & 27.12 & 25.679 & 5.31 \\
\hline No. 11 & 23.90 & 22.390 & 6.32 \\
\hline No. 12 & 10.39 & 9.145 & 11.98 \\
\hline No. 13 & 16.75 & 19.237 & -14.85 \\
\hline No. 14 & 11.67 & 13.442 & -15.19 \\
\hline
\end{tabular}




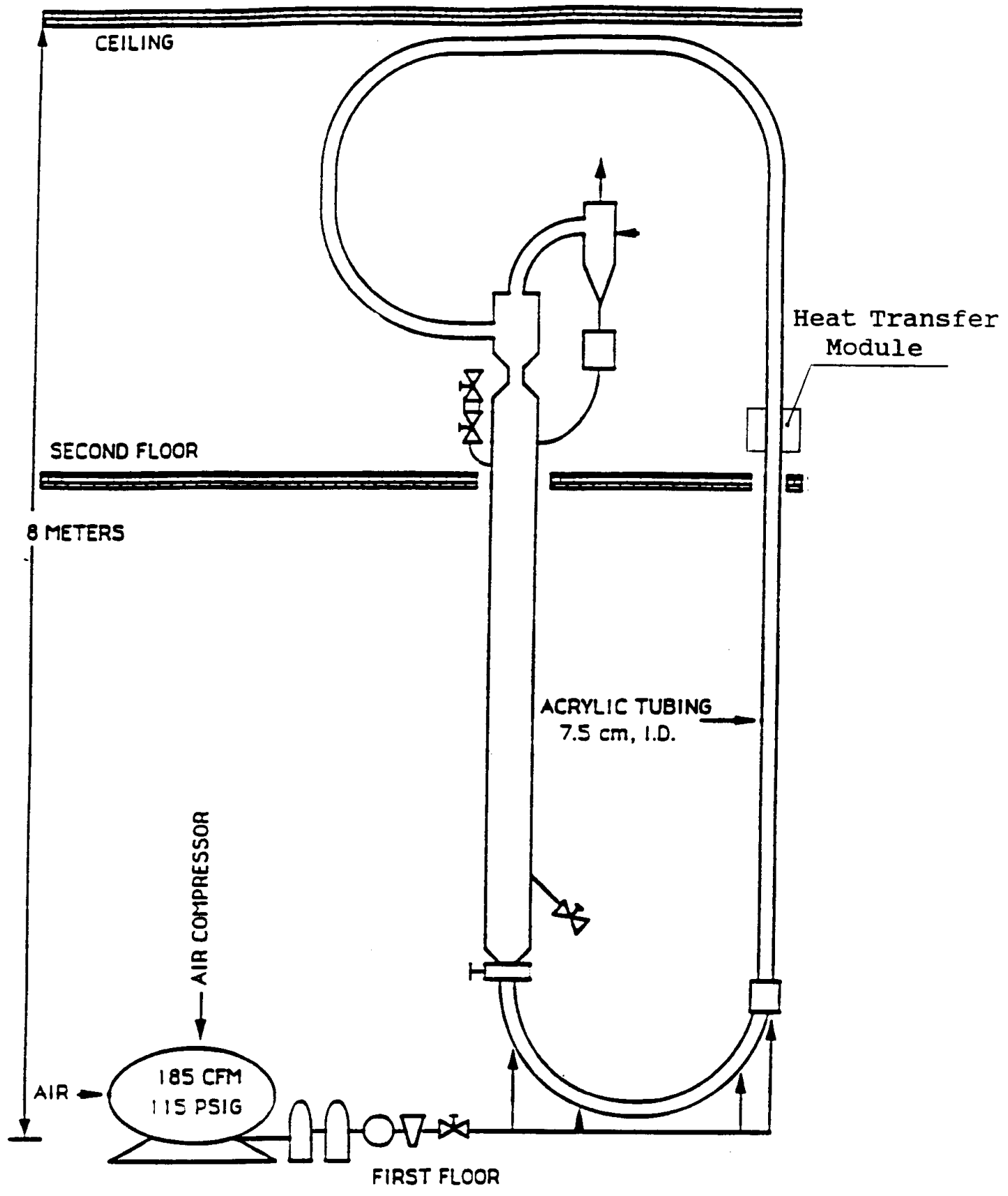

Figure 1 IIT Circulating Fluidized Bed System 

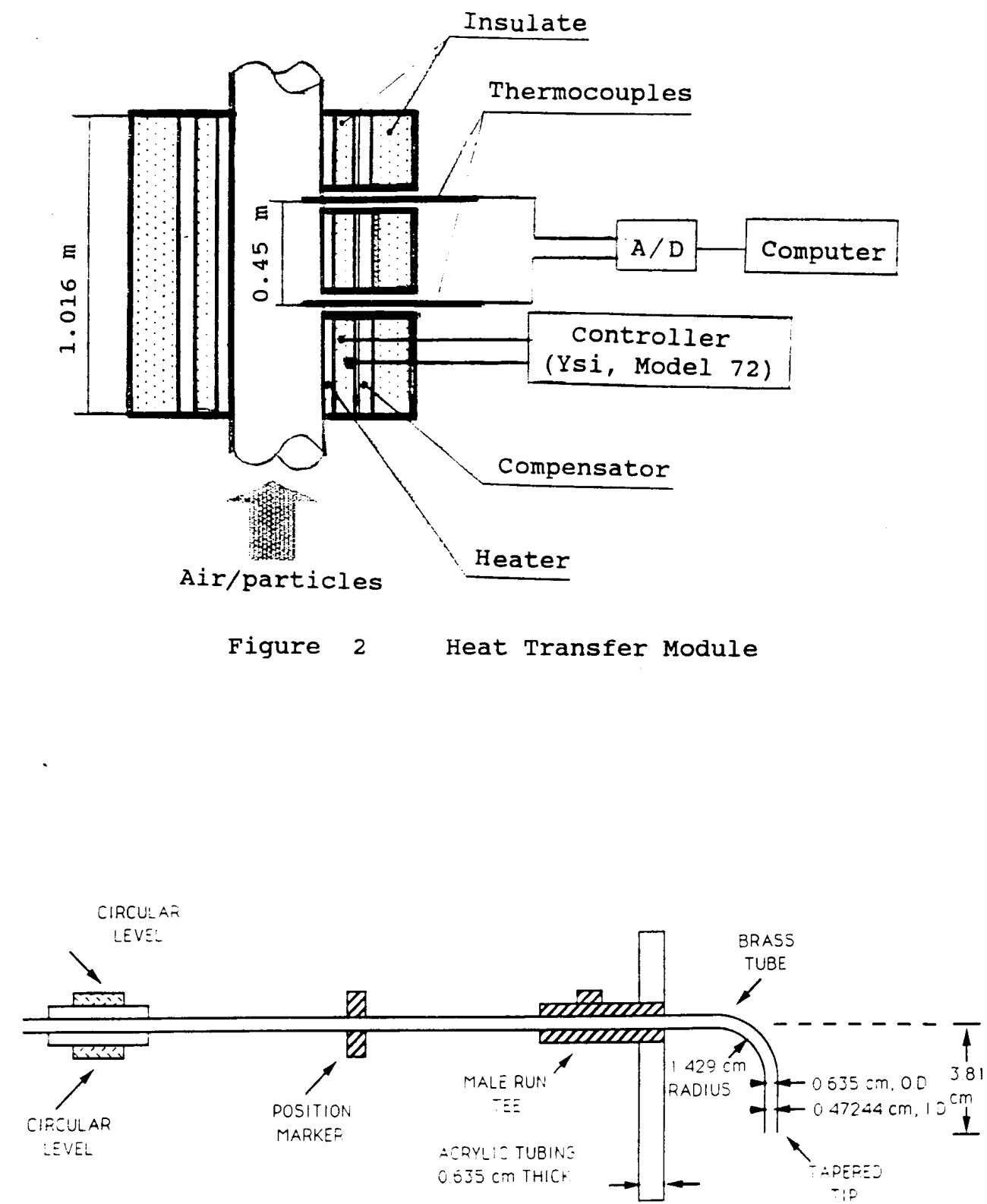

$22.86 \mathrm{~cm}$

Figure 3 Extraction Probe 


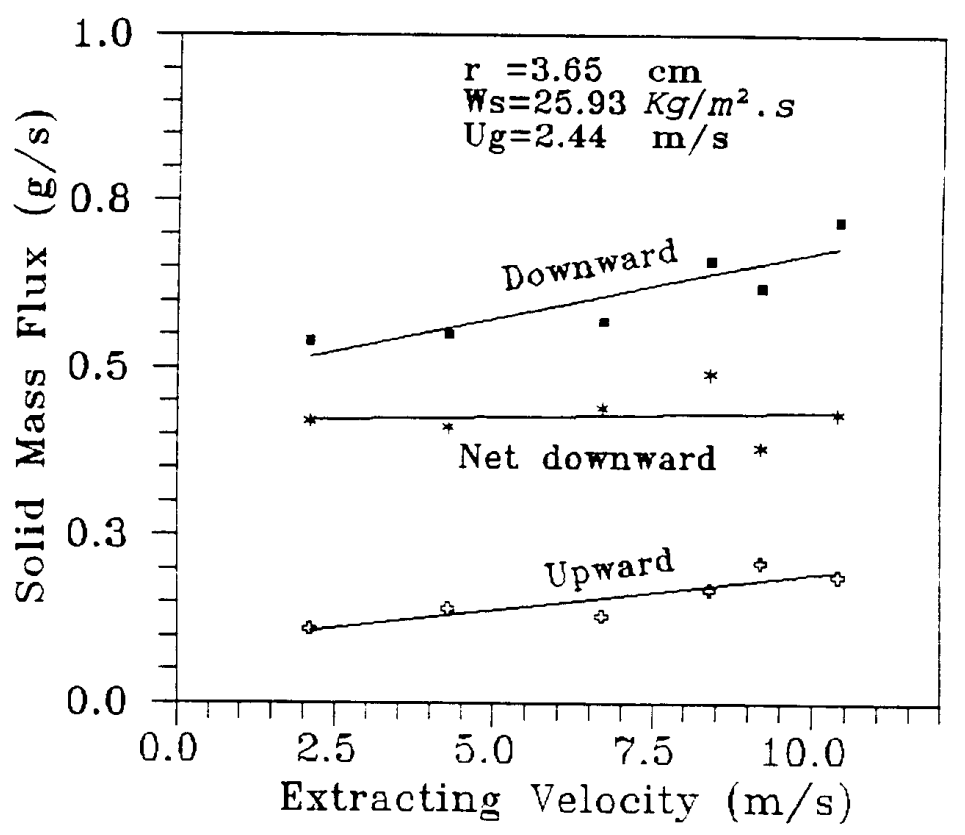

Figure 4 a Influence Extracting Velocity on The solid Mass Flux

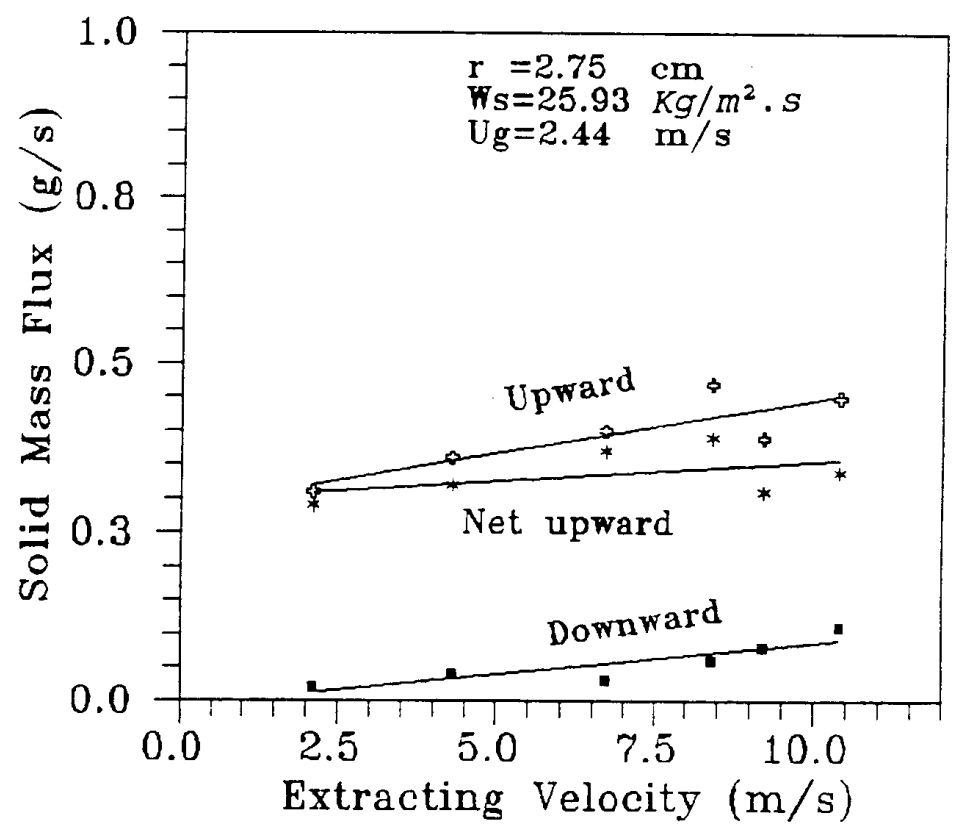

Figure $4 \mathrm{~b}$ Influence Extracting Velocity on The Solid Mass Flux 


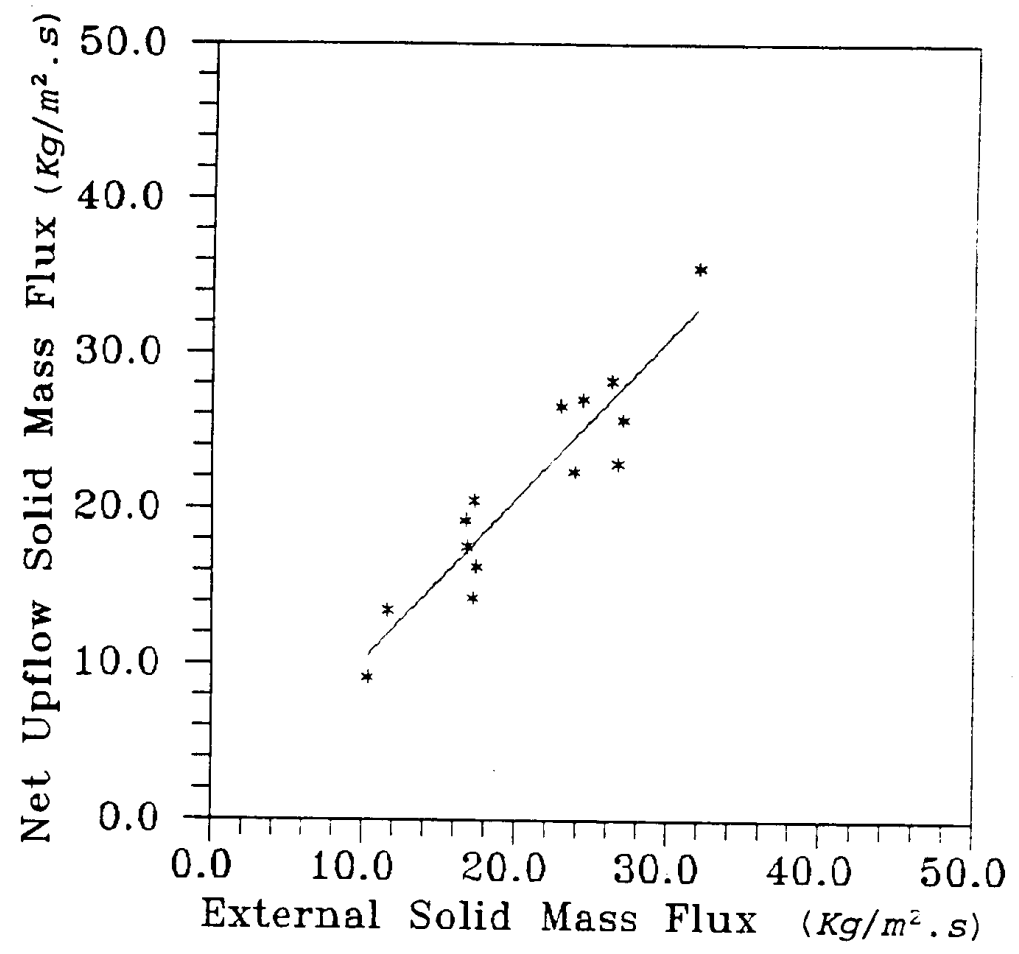

Figure $4 \mathrm{C}$ Comparision between Net Solid Mass Flux and External Solid Mass Flux

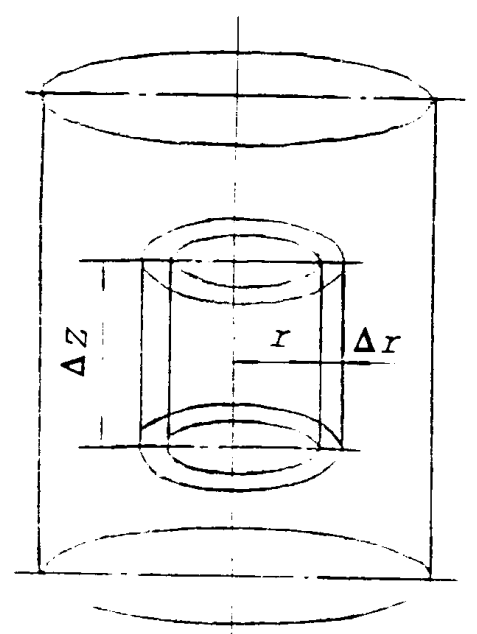

Figure 5 A Differential Annular section 


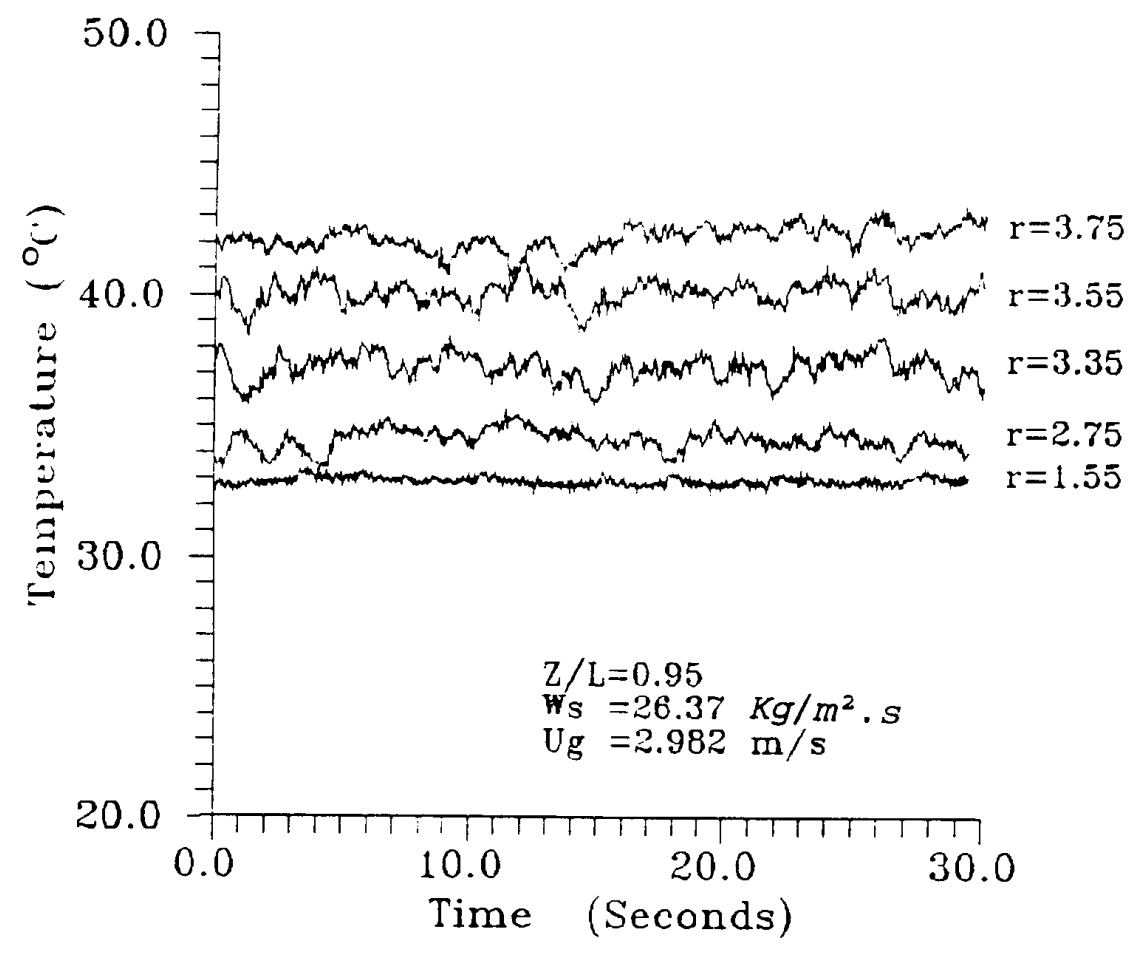

Figure $6_{a}$ Local Instantaneous Temperature Fluctuations

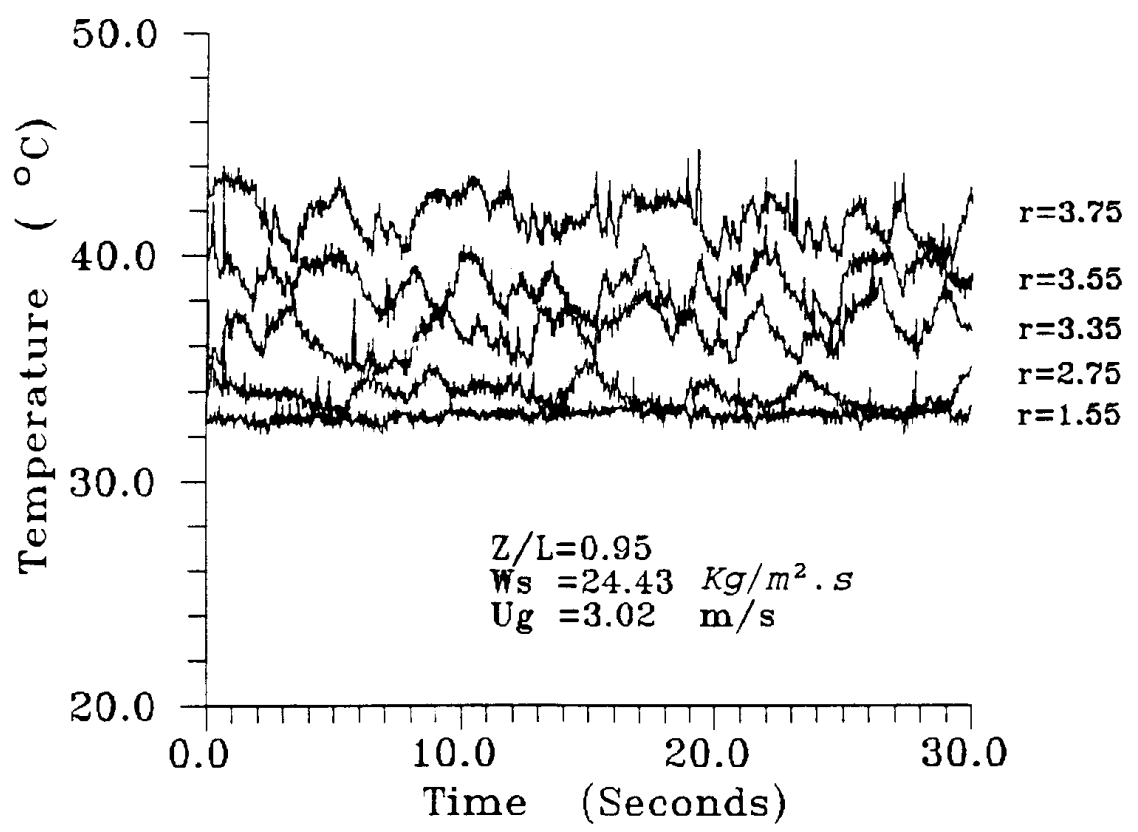

Figure $6_{b}$ Local Instantaneous Temperature Fluctuations 


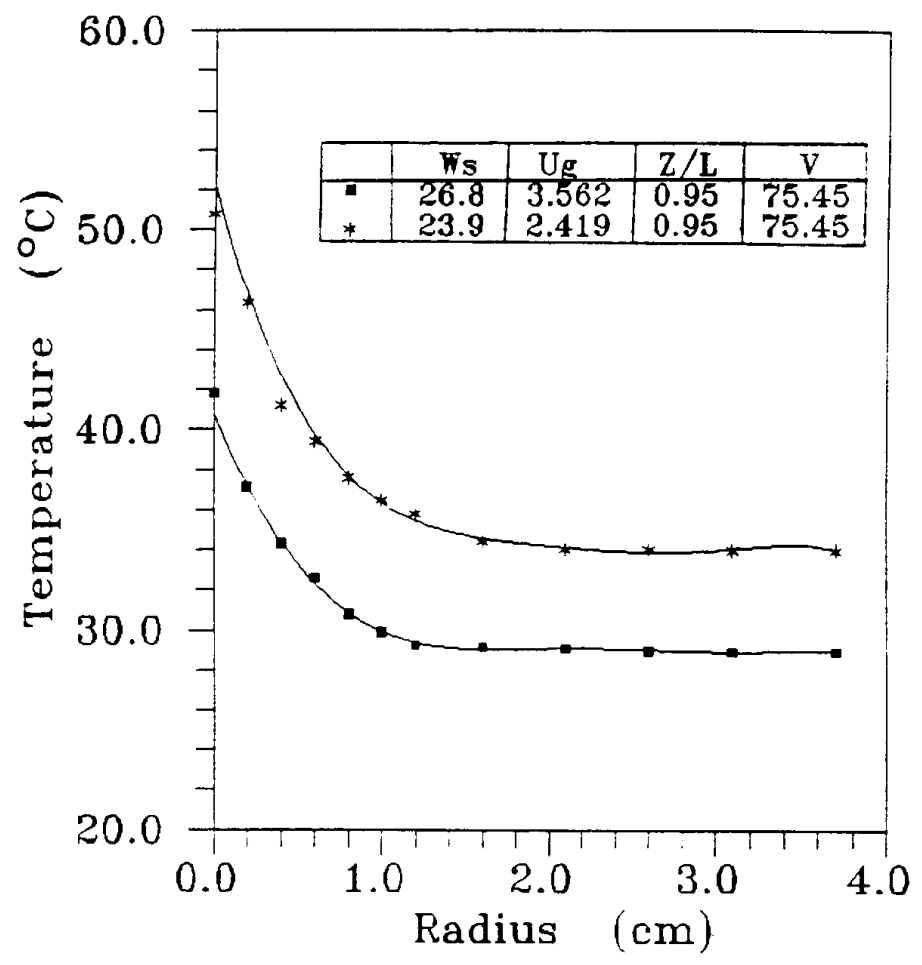

Figure 7a Radial Time-averaged Temperature Distributions

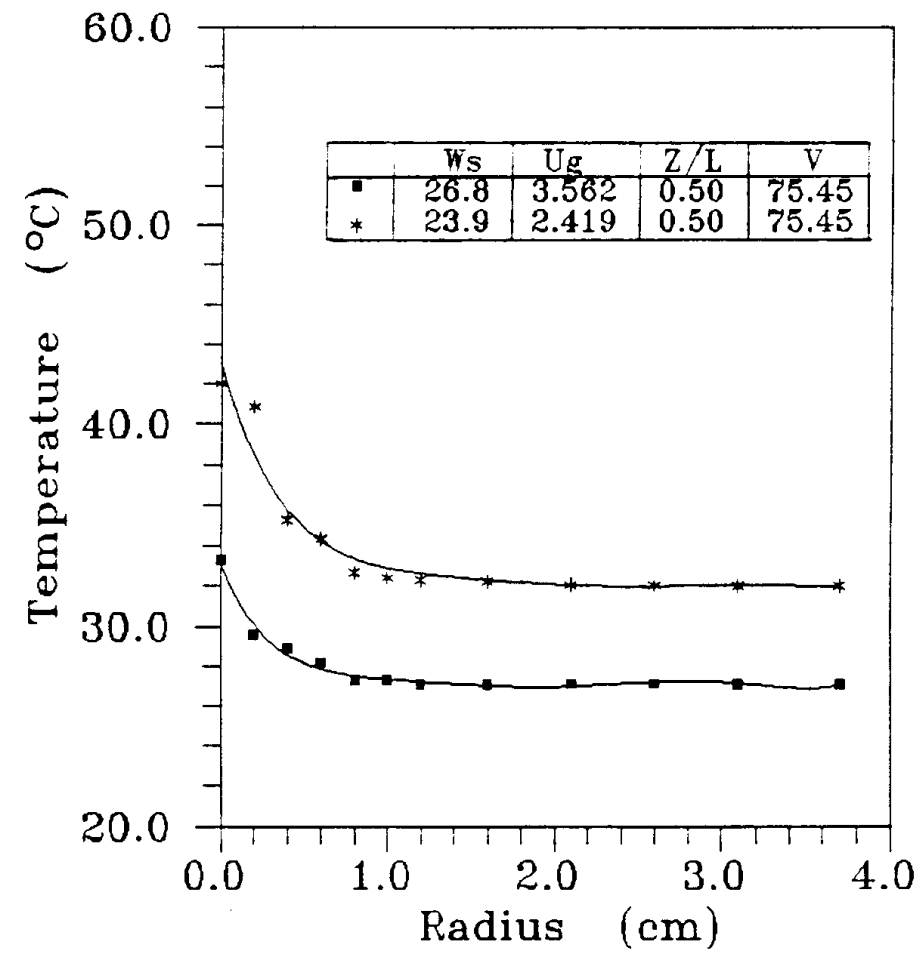

Figure $7 \mathrm{~b}$ Radial Time-averaged Temperature Distributions 


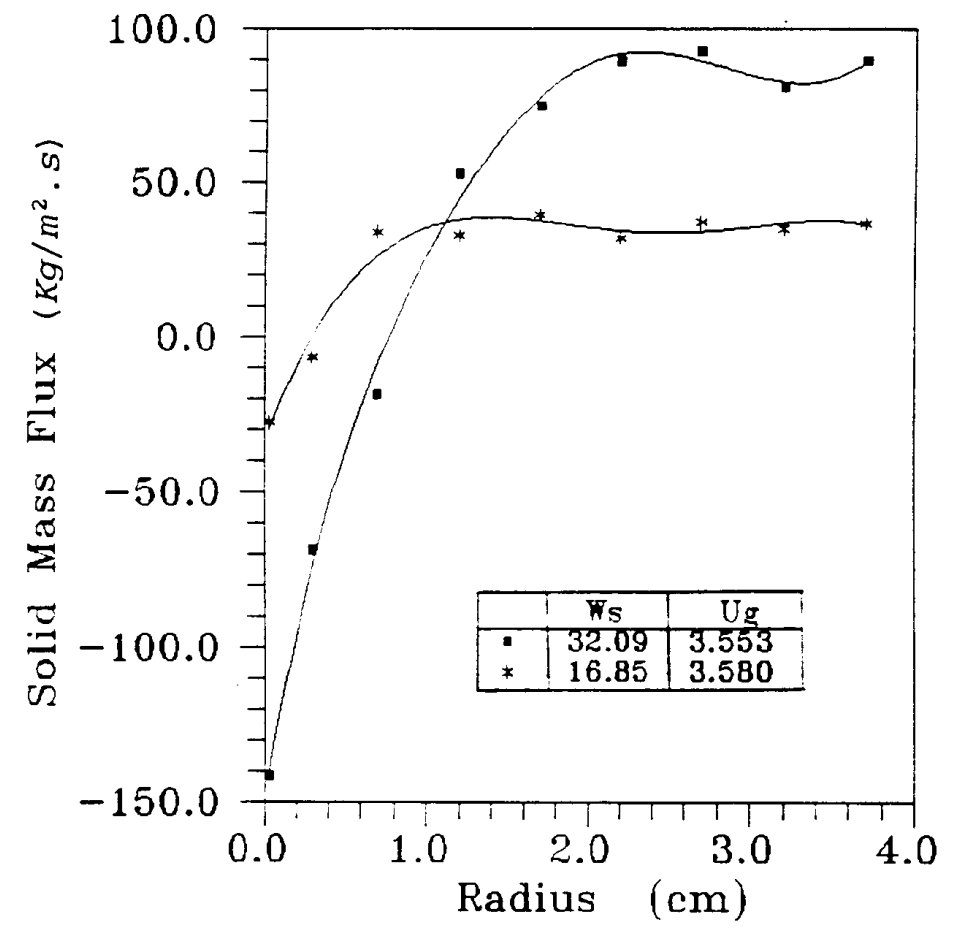

Figure 8 Radial Solid Mass Flux for Various Solid Feed Rates

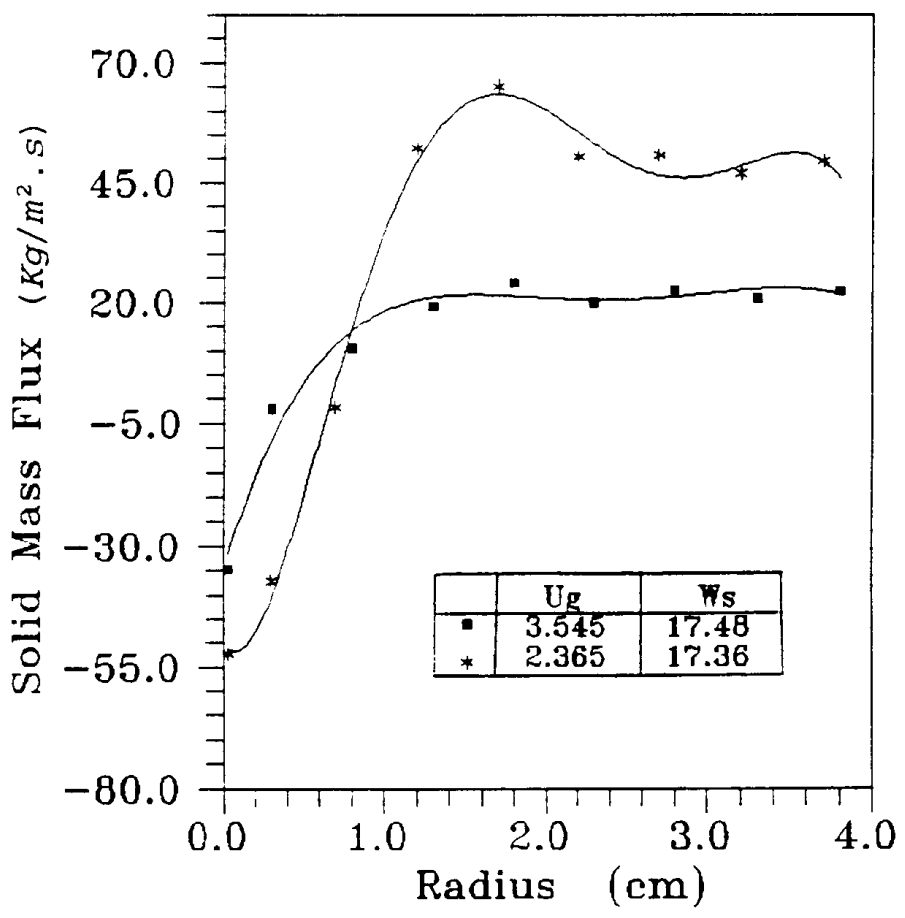

Figure 9 Radial Solid Mass Flux for Various Velocities 


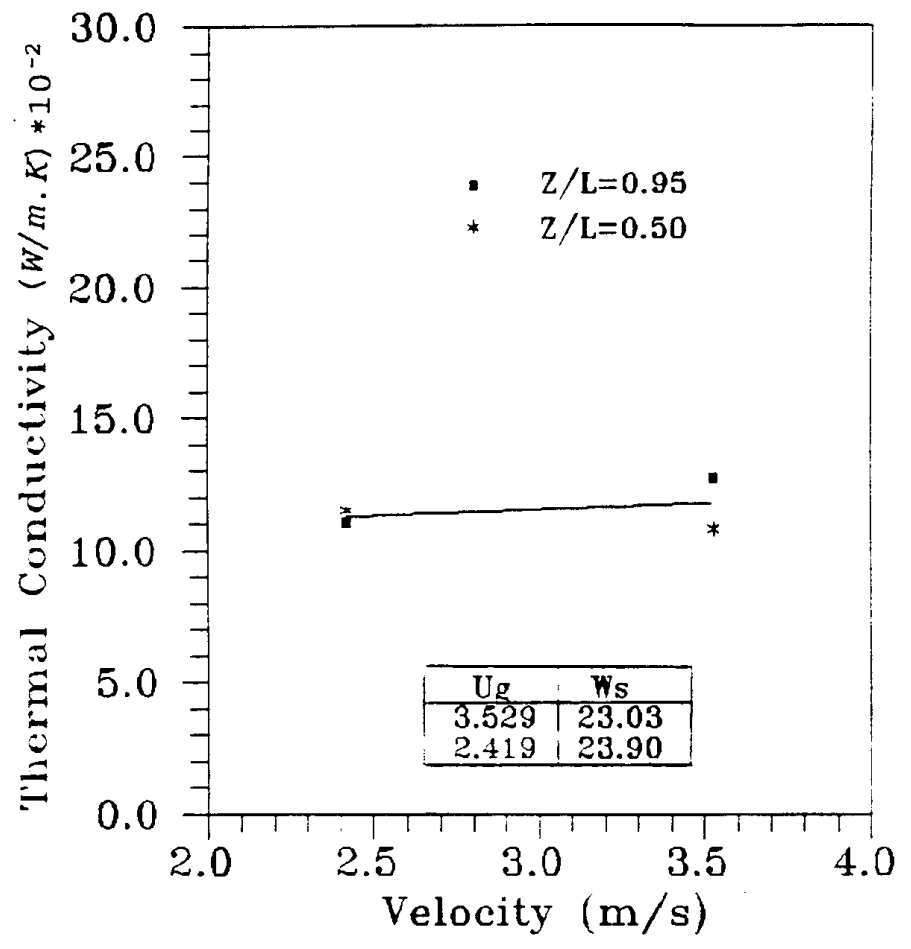

Figure 10 Effective Thermal Conductivity for Various Velocities

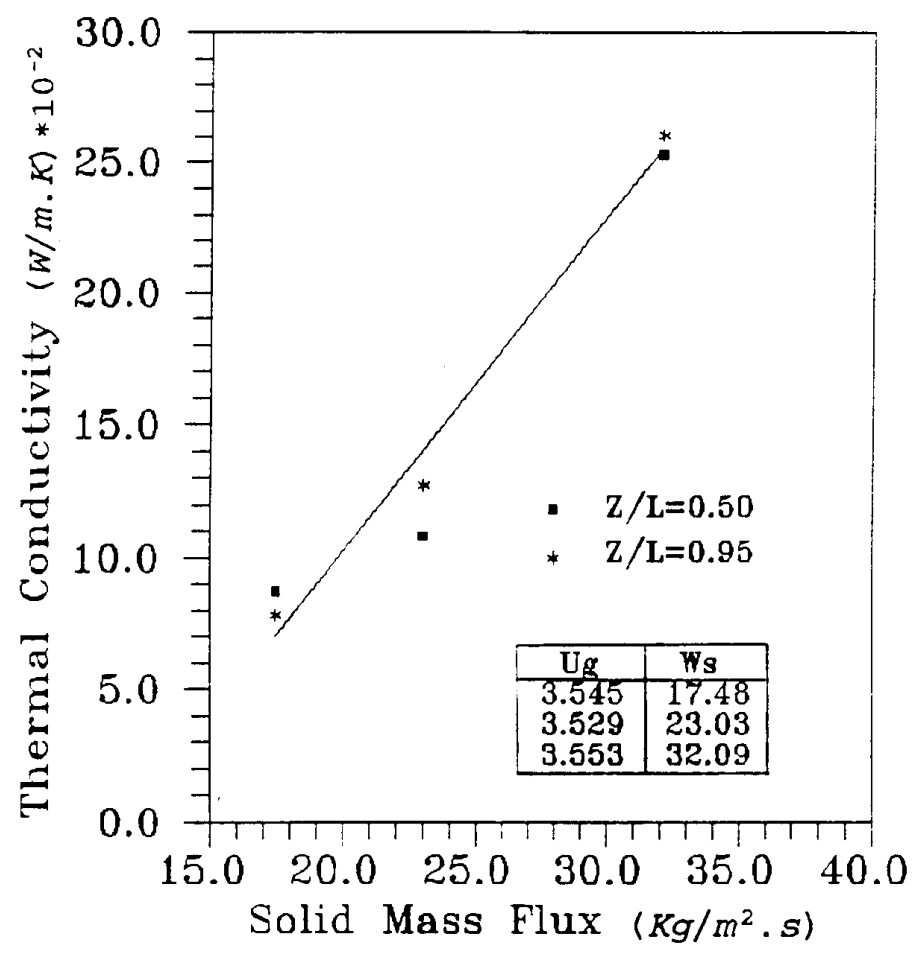

Figure 11 Effective Thermal Conductivity for Various Solid Fluxes 


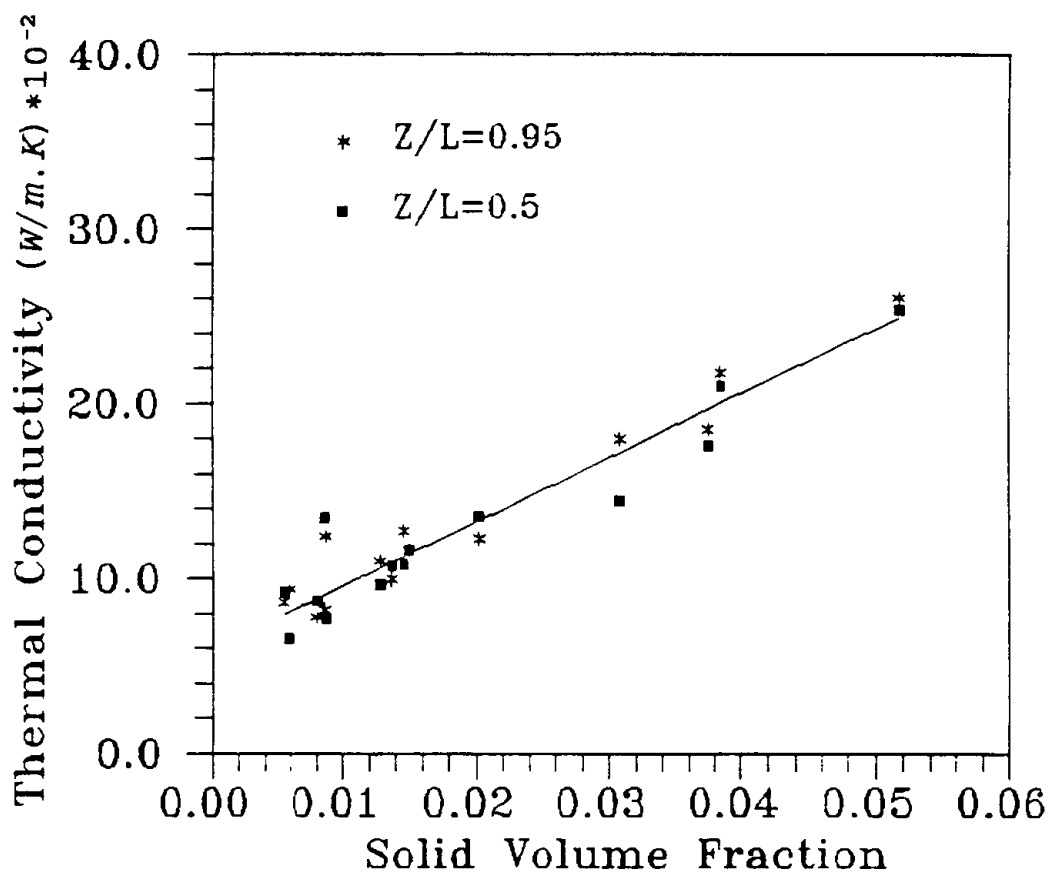

Figure 12 Thermal Conductivity Correction

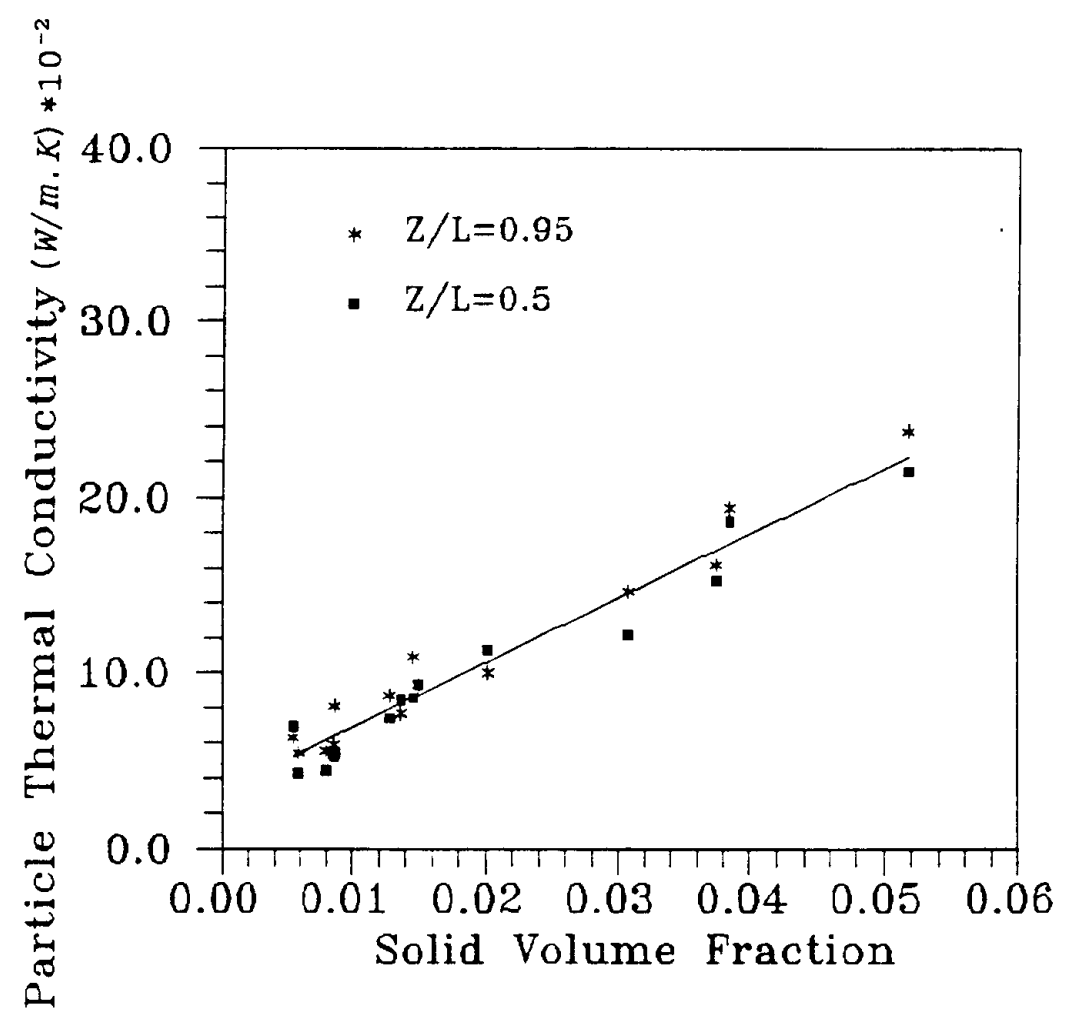

Figure 13 Particle Thermal Conductivity Correction 


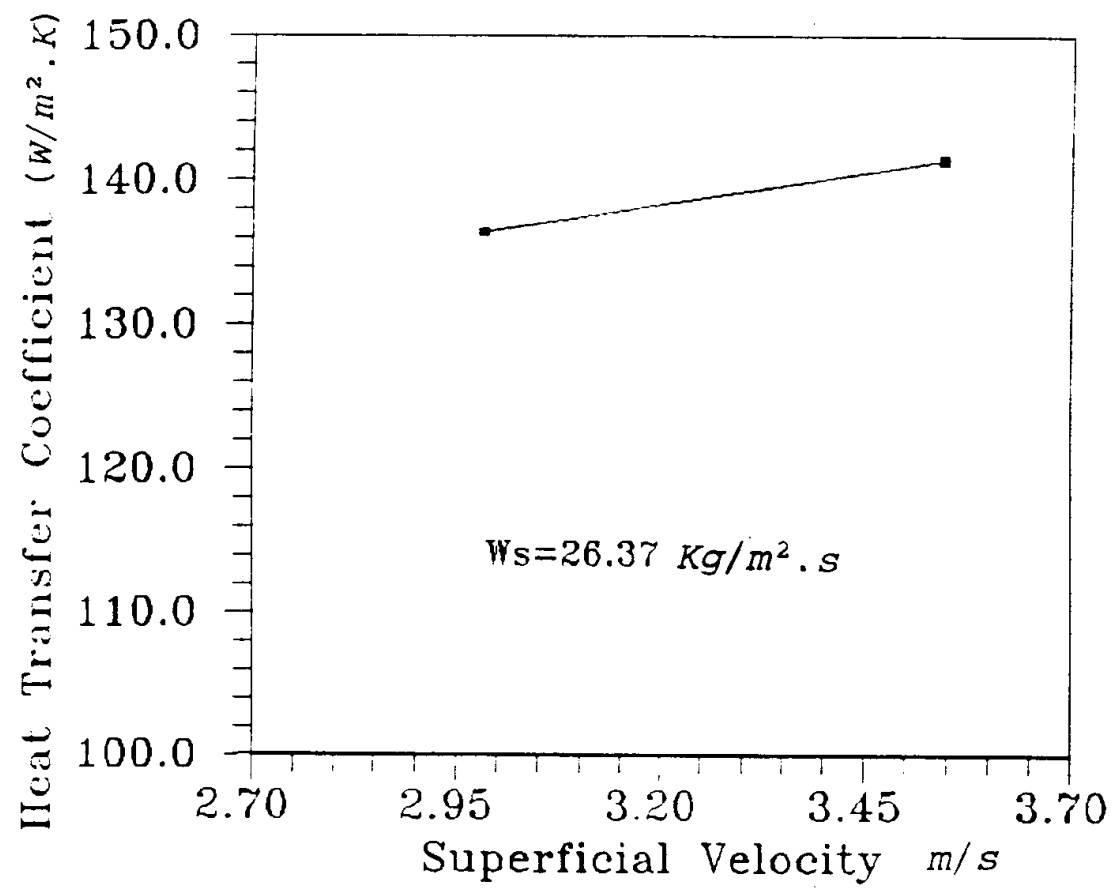

Figure 14 Effect of Velocity on Transfer Coefficient

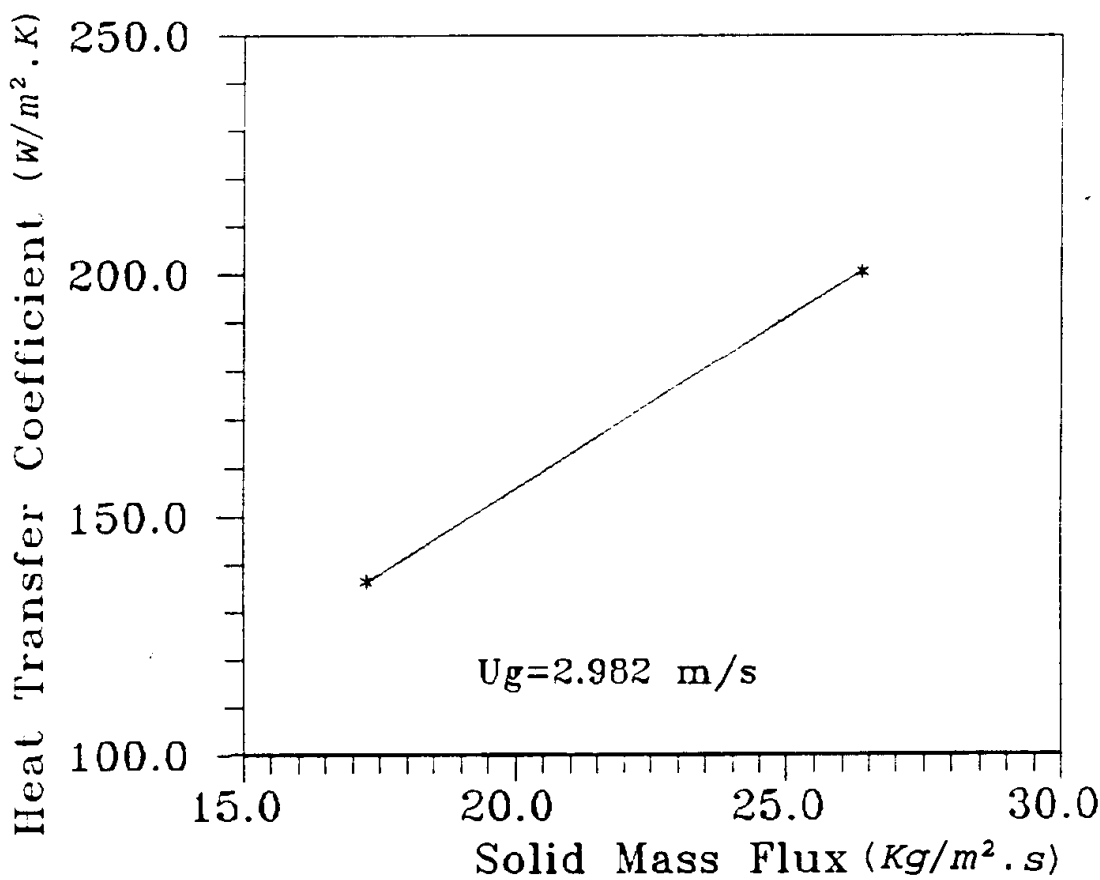

Figure 15 Effect of Solid Flux on the Heat Transfer Coefficient 


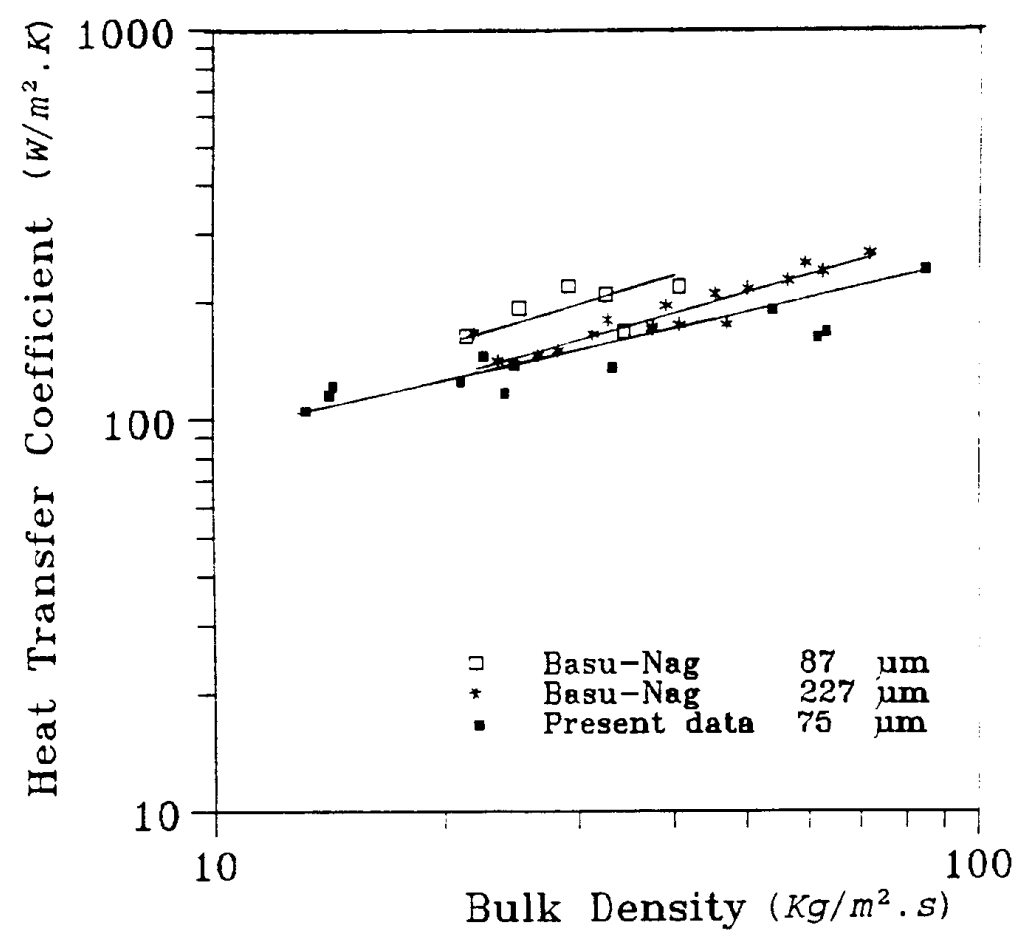

Figure 16 Effect of Bulk Density on the Heat Transfer Coefficient

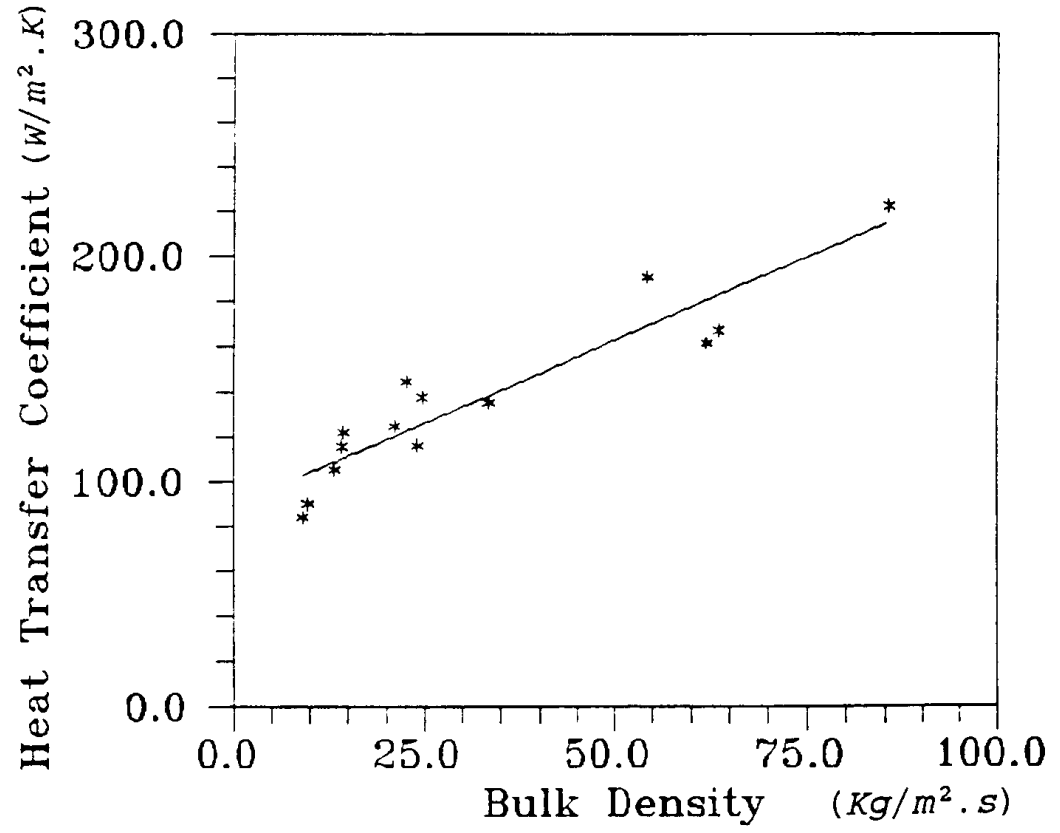

Figure 17 Effect of Bulk Density on the Heat Transfer Coefficient 


\title{
Large Scale Oscillations or Gravity Waves in Risers and Bubbling Beds
}

\author{
Dimitri Gidaspow, Lu Huilin and Reza Mostofi \\ Department of Chemical and Environmental Engineering \\ Illinois Institute of Technology \\ Chicago, IL. 60616
}

\begin{abstract}
An important characteristic of fluidized beds is that they never reach a steady state. To explain the behavior, the classical wave theory is applied to fluidization by replacing the acoustic sonic velocity with the square root of the derivative of the solids pressure with respect to density. This pseudo-sonic velocity is obtained from measurements of granular temperature and an equation of state for the cracking catalyst that has been established in an earlier publication.

The wide range of granular temperature is explained in terms of a new analytical solution of the problem. The wave theory gives a resonant frequency and shows the possibility of the existence of standing waves which set up systems of steady vortices sometimes computed in the complete numerical solution of the Navier-Stokes type equations for fluidization.

A gamma ray densitometer was used to measure axial porosity oscillations in a two story circulating fluidized bed for flow of $75 \mu \mathrm{m}$ cracking catalyst particles. In the upper portion of the riser, there was a distinct dominant frequency of less than $0.2 \mathrm{Hertz}$ which decreases with increasing porosity. In the lower portion of the tube the behavior was more chaotic and the dominant frequency was less defined. It approached one Hertz for porosity of 0.6 .

To explain these data and the bubbling bed frequencies an analytical expression for the dominant frequency of oscillations was developed starting with conservation of mass and momentum. In agreement with riser measurement it decreases to zero, as the solid volume fraction decreases. It agrees with the decrease of the bubbling bed frequency with bed height. For incompressible flow, the large scale oscillation is simply the classical gravity wave obtained from the transient Bernoulli equation.
\end{abstract}




\section{Introduction}

The understanding and prediction of large scale oscillations of gas-solid flow, sometimes referred to as hydrodynamic instability (Jackson, 1985), is of interest in nature, such as in the explosive volcanic eruptions (Neri, et al 1998), industrially in coal combustors and in fluidized catalytic cracking (FCC) reactors (Squires, et al., 1985) and in the control of solid propellant rockets (Culik, 1994). Fluidized bed reactors are widely used industrially due to their excellent heat and mass transfer characteristics, which are caused by oscillations of particles. One can group these oscillations into large-scale oscillations, which are of the order of equipment size, reactor height or diameter, and small-scale oscillations that are of the order of particle size.

\section{1a. Solids Viscosity and Kinetic Theory Models}

Recent reviews (Gidaspow, 1994; Jackson, 1993; Sinclair, 1997; Kuipers, et al, 1998) show that two types of Navier-Stokes type models were developed to simulate fluidized beds and compared with experiments. The model with solids viscosity as an input was used by Tsuo and Gidaspow (1989), Benyahia, et al (1998) and by Sun and Gidaspow (1999) to model riser (vertical pipe) flow. Bubbling flow was modeled similarly by Lyckowski, et al (1993) and by Anderson, et al (1995). These models compute the large-scale density oscillations which we refer to as gravity waves. However, for riser flow they have not been compared to experimental density fluctuations due to absence of such data. This paper presents such data.

The second Navier-Stokes type model is based on the granular theory of Savage and co-workers (Lun, et al 1984; Savage 1988). It was applied to steady, developed riser flow in a pioneering paper by Sinclair and Jackson (1989) using the Johnson and Jackson (1987) boundary conditions. Developed flow requires the solids pressure to be constant.

For an ideal type equation of state for particles, verified experimentally by Gidaspow and Huilin (1998) for FCC particles, this approximation states that the product of the granular temperature (essentially, oscillating kinetic energy of particles) and the solids volume fraction is a constant. But with a restitution coefficient of near unity and the Johnson and Jackson (1987) boundary conditions the granular temperature equation shows that the maximum in the granular temperature is at the center of the riser. In view of the constancy of the granular pressure, the solids volume fraction is a minimum at the pipe center. This leads to the observed core-annular flow for both riser flow and for the cocurrent down flow. The viscosity input riser model also leads to the core-annular flow regime. In this transient model the transfer of particles to the wall is due to the large-scale oscillations. The accumulation of particles at the wall is due to the low gas velocity at the wall which is insufficient to maintain the upward flow of particles.

It was only a decade ago that the oil industry using gamma ray techniques (Sun and Koves, 1998) learned that their large diameter risers operate in the core-annular flow regime: the core is very dilute. The core-annular structure leads to two main problems: (1) insufficient gas-solids contact, and (2) back-mixing due to non-uniform radial distributions (Jin, et al 1997). This unfavorable radial volume fraction distribution of solids in the riser has led to consideration of new schemes of contacting for a refinery of the $21^{\text {st }}$ century.

The transient kinetic theory model (Gidaspow, 1994; Samuelsberg and Hjertager, 1996 a \& b) also requires some experimental input. For gas-solid flow the main input is 
the restitution coefficient which is a function of velocity at impact. At the present time there is no way of accurately estimating the restitution coefficient. Hence the granular temperature was experimentally measured for FCC particles using a CCD camera and the equation of state for particles was constructed using the measured solids pressure and the radial distribution functions of statistical mechanics (Gidaspow and Huilin, 1996 \& 1998). The kinetic theory model clearly explains why fluidized beds are such good heat transfer devices. Their thermal conductivity, $k$ can be expressed in terms of mean free path, $l$, the average of fluctuating velocity, $\langle v\rangle$ which is essentially the square root of the granular temperature and the density and the specific heat of particles as follows.

$$
k=l\langle v\rangle \rho C_{p}
$$

where $\rho=$ density and $C_{p}=$ heat capacity of particles.

The mean free path, $l$ is essentially the particle diameter divided by the solids volume fraction. For dense systems it is of the order of particle diameter. For dilute conditions it will approach the size of equipment. The granular temperature rises with the particle concentration, reaches a maximum near about five percent solids and then decreases due to a decrease in the mean free path. Cody's data (Buyevich and Cody, 1998) suggest that the granular temperature have a maximum for a particle size of about 90 microns, close to the size of FCC particles. Hence the thermal conductivity should have a maximum at this particle size, as indeed it appears to have. We see that the kinetic theory model has the potential to explain why fluidized beds are such good contacting devices.

\section{1b. Sonic Velocity of Particles}

The granular flow kinetic theory also helps to explain the mechanism of largescale wave propagation. From the work of Savage (1988) and others we know that density waves move with the pseudo sonic velocity, the derivative of solids pressure with respect to the density. Hence we present our data for sonic velocities for FCC particles.

The large-scale oscillation frequency is the sonic velocity divided by the equipment size. Near five percent solids and less the collisional and the cohesive contributions in the equation of state are negligible. Then the sonic velocity is simply the square root of the granular temperature. For FCC particles it is of the order of one meter

per second versus about $300 \mathrm{~m} / \mathrm{s}$ for gases. Hence for particles the oscillations associated with the hydrodynamic instability are of the order of one Hertz for particles versus 300 for air for a one meter length.

\section{1c. Resonant Vibrations}

Resonant vibrations that are vibrations whose natural frequency equals the forced frequency are known to set up large amplitudes of oscillations. The natural frequency can be interpreted to be as the frequency obtained from the sonic velocity discussed above. The forced frequency is the gravity wave obtained in this paper. It agrees with literature bubbling bed data and with the riser data presented here.

The scale-up of the circulating fluidized bed reactor for making liquids from synthesis gas, the Synthol reactor was delayed by two years due to vibrations caused by the dense iron catalyst not encountered in the much smaller pilot plant (Matsen, 1997; Shingles and Mc Donald, 1988). We may speculate that resonance was set up when the 
length was made larger, since the catalyst concentration was probably the same in the pilot plant and in the commercial unit.

\section{IIT Circulating Fluidized Bed}

The experimental set-up is shown in Figure 1a. The riser is an acrylic tube of 0.07 $\mathrm{m}$ ID and a height of $6.58 \mathrm{~m}$. Fluid cracking catalyst particles of a density of $1654 \mathrm{~kg} / \mathrm{m}^{3}$ and an average diameter of $75 \mu \mathrm{m}$ were used. The CFB and the particles are identical to those used by Miller and Gidaspow (1992). Charge is removed by a metallic wire along the wall of the tube. Figure $1 \mathrm{~b}$ shows a sketch of time average particle concentration and flux profiles obtained by Miller and Gidaspow (1992). A video made previously by Miller (1992) shows that in the dense regime of interest in this study the particles at the wall undergo a vigorous up and down motion. It is basically this random particle motion that we measured with our CCD camera and converted into granular temperature (Gidaspow and Huilin, 1996). From it we had obtained a collisional viscosity that matched Miller and Gidaspow's (1992) reported viscosity obtained from pressure dropradial particle velocity measurements.

Porosity measurements across the whole tube were made at a height of $1.83 \mathrm{~m}$ on the first floor and the second floor at a height of $4.4 \mathrm{~m}$ with a gamma ray densitometer with a collimation diameter of about one centimeter.

\section{Gamma Ray Densitometer}

A gamma ray densitometer has been successfully used to understand the dynamics of bubbling fluidized beds for at least the last 34 years (Baumgarten et. al, 1960; Weimer et al., 1985; Ding et. al., 1990). It has surprisingly not been applied to understand the dynamics of a CFB despite its potential use in industrial risers and high pressure combustors. In this study a gamma ray densitometer equipped with a scintillation detector was used to measure porosity oscillations in the IIT CFB, shown in Fig. $1_{\mathrm{a}}$, at two locations. In the bubbling bed study of Tsinontides and Jackson (1993), they used a radioactive beam of only a few millimeter diameter to have a good spatial resolution. Such a small beam does not allow the transient determination of porosities. The beam in this experiment was one centimeter.

The gamma ray densitometer was used to measure instantaneous averaged crosssectional porosities. The source is a $500 \mathrm{mCi}-\mathrm{Cs}-137$ source having a single gamma ray of $667 \mathrm{Kev}$ and a half life of 30 years. The Cs-137 was sealed in a welded stainless steel capsule. The source holder was welded, filled with lead, and provided with a shutter to turn off the source. The detector is a NaI (Ti) crystal scintillation detector (Teledyne, ISOTOPESS-44-I/2). It consisted of a $2 \mathrm{~mm}$ thick, $5.08 \mathrm{~cm}$ diameter tube with a $0.14 \mathrm{~mm}$ thick Beryllium window. The transmitted radiation was converted to electrical pules by photo-multiplier (Model 266, EG\&G Ortec). The converted electrical signals were passed through a series of data conditions including a preamplifier (Model 113, EG\&G), an amplifier and a double channel analyzer (model 778). The DCA has been used to remove low energy level noises. A DCA produces an output logic pulse only if the peak amplitude of this input signal falls within the energy window that is established with two preset threshold levels. The output of DCA connects to a modular data acquisition and control system (ISAAC 91-I) which was used as the interface between the densitometer and personal computer. The output signal of DCA is sent into a counter device. The 
counter counts down from 65535 to zero and reinitializes automatically. The counting number is sent to computer in binary form and managed by a PC computer. An IBM PC with a $20 \mathrm{MB}$ hard disk and a Math coprocessor has been used to store and analyze data. The data analysis consisted of transient state porosity and steady state porosity measurement. The power spectrum is calculated by using the fast Fourier transform (FFT) method. The intensity of transmitted radiation follows Beer's law. The attenuation coefficient for the particles was $0.02477 \mathrm{~cm} / \mathrm{g}$. A calibration curve was obtained by measuring radiation counts for an empty tube and one filled with particles. At a porosity of 0.4 the count was 22400 while for the empty tube it was 23800 per second.

\section{Oscillations in the Dilute Regime}

Figure 2 shows the experimental porosity oscillations in the upper portion of this riser. As in the computation of the Pyropower system (Therdthianwong and Gidaspow, 1994), we see wild porosity oscillations depicted in Figure 2. Similar data were obtained at other gas velocities and solid fluxes. Figure 3 shows the spectral representation of the data in Figure 2. As in the simulation for the Pyropower type CFB (Gidaspow et al., 1995 ) there are two distinct peaks with little noise. Figure 4 shows a summary of spectra for other flow conditions. Figure 5 shows a plot of the dominant frequency as a function of porosity. The frequency is well below one Hertz, typical of bubbling beds. It increases linearly from 0.05 Hertz at a superficial gas velocity of $1.6 \mathrm{~m} / \mathrm{s}$ and a flux of $27.8 \mathrm{~kg} / \mathrm{m}^{2}$ $\mathrm{s}$ to 0.128 at a velocity of $2.6 \mathrm{~m} / \mathrm{s}$. It also increases linearly with solid flux from 0.06

Hertz at a flux of $18.5 \mathrm{~kg} / \mathrm{m}^{2}$-s at a velocity of $2.67 \mathrm{~m} / \mathrm{s}$ to $0.2 \mathrm{Hertz}$ at a flux of 34 $\mathrm{kg} / \mathrm{m}^{2}$-s. Such low frequencies were also obtained for pressure oscillations and analyzed by Bouillard and Miller (1994). The numerical simulation for the large scale unit (Gidaspow et al., 1995) operating at an order of magnitude larger flux shows that linear extrapolations of these data are not valid. The trend is in the right direction, however. The frequency increases to one Hertz for the higher gas and solid flows.

\section{Oscillations in the Dense Regime}

Figure 6 shows typical density fluctuations in the lower portion of the IIT CFB, $1.83 \mathrm{~m}$ above the inlet. In agreement with computations for the Pyropower system (Gidaspow et al., 1995) for an instant of time the average porosity in this region is near 0.8. The corresponding power spectrum depicted in Figure 7 shows a much more chaotic behavior. Other data show more pronounced dominant peaks summarized in Figure 8. The large scatter of the data is due to the absence of a distinct structure of flow in the bottom section of the CFB.

\section{Wave Propagation in Dense Flow}

The theory of fluidization, as described by Gidaspow (1994), shows that the mechanism of large scale oscillations is a coupled pressure and density non-linear wave propagation phenomenon. The small scale turbulence is described by the granular temperature, for which an additional conservation equation is written. For a very dense bed, such as a bubbling bed, a simplified theory shows that the density and the flux obey a simple wave equation with densities propagating at pseudo-sonic velocities of solids, $\mathrm{C}_{\mathrm{S}}$. The kinetic theory of granular flow shows that $\mathrm{C}_{\mathrm{S}}$ is the oscillating velocity of the particles. 
For the dense riser flow of interest here, the more complete equations must be considered. A Reynolds number criterion shows that it is sufficient to consider the particle equations only. For pipe flow with small catalyst particles the slip between the gas and the particles is small. Then the mixture equations show that the characteristic group is a mixture Reynolds number (Gidaspow, 1994). A useful Reynolds number, $R_{\mathrm{e}}$ is one based on some average velocity, $\mathrm{v}$ and, say, the pipe diameter, $\mathrm{D}$,

$$
R_{e}=\frac{D \rho_{m} v}{\mu_{p}+\mu_{g}}
$$

where $\mu_{p}$ is the particle viscosity which exceeds the fluid viscosity, $\mu_{g}$ by several orders of magnitude. The mixture density, $\rho_{m}$ is

$$
\rho_{m}=(1-\varepsilon) \rho_{s}+\varepsilon \rho_{g}
$$

For dense flow

$$
(1-\varepsilon) \rho_{s}>>\varepsilon \rho_{g}
$$

For $\rho_{s} / \rho_{g}=1000$

$$
1-\varepsilon>0.001
$$

for $\varepsilon_{s}$, the volume fraction of solids of much greater than $0.1 \%$. Thus for particle concentration of much greater than $0.1 \%$, the Reynolds number criterion is

$$
R_{e}=\frac{D(1-\varepsilon) \rho_{s} v}{\mu_{p}}
$$

It does not involve gas properties. Hence it is sufficient to consider the particle equations only. The one dimensional particle mass and momentum balances are as follows:

Solid Mass Phase Balance:

$$
\frac{\partial}{\partial t}\left(\varepsilon_{s} \rho_{s}\right)+\frac{\partial}{\partial x}\left(\varepsilon_{s} \rho_{s} v_{s}\right)=0
$$

Inviscid Momentum Balance:

$$
\frac{\partial}{\partial t}\left(\varepsilon_{s} \rho_{s} v_{s}\right)+\frac{\partial}{\partial x}\left(\varepsilon_{s} \rho_{s} v_{s} v_{s}\right)=-\frac{\partial p_{s}}{\partial x}
$$

The theory of characteristics (Gidaspow, 1994) shows that the characteristic directions are

$$
v_{s} \pm C_{s}
$$

where the pseudo-sonic velocity (Savage, 1988) is

$$
C_{s}=\left(\frac{\partial p_{s}}{\rho_{s} \partial \varepsilon_{s}}\right)^{\frac{1}{2}}
$$

Ocone and Astarita (1994) obtained a similar expression of the pseudo-sonic velocity assuming a reversible and pseudo-adiabatic process. Particle waves move with velocities given by Eq. (8). The difficulty with this theory is that the particle sonic speed must be known. In the next section we will show that it can be obtained from measurements of granular temperature.

For more dilute flow, as in pneumatic transport, the more complete equations must be considered. Gidaspow (1994) shows that the characteristic paths for density, pressure, etc. wave propagation are: 


$$
\begin{gathered}
v_{g} \pm C_{g} \\
\text { and } \\
v_{s} \pm C_{s}
\end{gathered}
$$

\section{Particle Sonic Velocity}

Particle sonic velocity can be estimated based on kinetic theory derived equation of state and measurements of granular temperature in the CFB. Granular temperatures were measured in the IIT CFB using a CCD camera (Gidaspow and Huilin, 1996). The technique involves a measurement of particle velocity distribution, in principle similar to that obtained much earlier by Carlos and Richardson (1968) using a photographic method. A particle makes a streak on a computer screen for a millisecond or so. The distances traveled divided by time give two velocity components. Many such measurements give distribution curves of the axial and radial velocities. Table A listed the experimental data in the dilute region. Since the radial velocities are small compared with the axial velocities, isotropy is a reasonable assumption in the radial direction. Such calculations and measurements give us the granular temperature depicted in Figure 9 (Gidaspow and Huilin, 1996).

The sonic velocity, $C_{s}$, as defined by Equation (9) is then obtained from the derivative of the equation of state for the particles originally derived by Lun et al. (1984):

$$
p_{s}=\varepsilon_{s} \rho_{s} \theta\left[1+2 \varepsilon_{s}(1+e) g_{0}\right]
$$

where we used Bagnold 's relation of $g_{0}$ given by (Bagnold, 1954):

$$
g_{0}=\left[1-\left(\frac{\varepsilon_{s}}{\varepsilon_{s, \max }}\right)^{\frac{1}{3}}\right]^{-1}
$$

Figure 10 shows the computed sonic velocities in our CFB. They are of the order of magnitude estimated by Gidaspow (1994) from measurements of discharge of particles from orifices. Figure 10 also shows the measured average particle velocities obtained using our digital video camera.

\section{Compressible Large Scale One Dimensional Oscillation}

In this section we derive an equation for the observed large scale low frequency oscillation in the vertical pipe. We begin with the momentum balance for the solids Equation (7.2) in Gidaspow's book. To obtain an analytical solution for the frequency, a number of approximations are necessary. First, we neglect the velocity square terms. This simplification will immediately lead to the neglect of $\mathrm{V}_{\mathrm{s}}$ compared to $\mathrm{C}_{\mathrm{s}}$ in the wave propagation analysis. Next we neglect the small contribution, for one dimensional analysis, of wall friction caused by solids viscosity. We further restrict the analysis to flow of particles above minimum fluidization. This leads to the neglect of solids stress caused by particle contact except those caused by collisions. With these simplifications the solids momentum balance for phase s becomes as follows. 


$$
\frac{\partial\left(\varepsilon_{s} \rho_{s} v_{s}\right)}{\partial t}=-\frac{\partial P_{s}}{\partial x}-\varepsilon_{s} g\left(\rho_{s}-\rho_{g}\right)-\beta\left(v_{s}-v_{g}\right)
$$

$\begin{array}{ll}\text { Acceleration of } & \text { Solids Buoyancy Drag } \\ \text { Momentun } & \text { Pressure due } \\ \text { of Phase } s & \text { to Collisions }\end{array}$

Note that for a steady state with a negligible solids pressure, Equation (13) is the conventional balance of buoyancy and drag. Here, however, we emphasize the time variation. Hence we need the transient conservation of particles equation, which is as follows in one dimension

$$
\frac{\partial\left(\varepsilon_{s} \rho_{s}\right)}{\partial t}+\frac{\partial\left(\varepsilon_{s} \rho_{s} v_{s}\right)}{\partial x}=0
$$

A wave equation for the bulk density $\rho_{B}=\rho_{s} \varepsilon_{s}$ or for the flux, $F=\rho_{s} \varepsilon_{s} v_{s}$ can be obtained by following the procedure in chapter 7 in Gidaspow's book. Differentiation of Equation (14) with respect to time gives

$$
\frac{\partial^{2} \rho_{B}}{\partial t^{2}}=-\frac{\partial^{2} F}{\partial t \partial x}
$$

while differentiation of Equation (13) with respect to $\mathrm{x}$ for $\rho_{s}>>\rho_{g}$ gives

$$
\frac{\partial^{2} F}{\partial x \partial t}=-C_{s}^{2} \frac{\partial^{2} \rho_{B}}{\partial x^{2}}-g \frac{\partial \rho_{B}}{\partial x}-\beta\left(\frac{\partial v_{s}}{\partial x}-\frac{\partial v_{g}}{\partial x}\right)
$$

Combination of Equations (15) and (16) produces the one-dimensional wave equation for bulk density, where $\mathrm{C}_{\mathrm{s}}$ was defined by Equation (9).

$$
\begin{aligned}
& \frac{\partial^{2} \rho_{B}}{\partial t^{2}}=C_{s}^{2} \frac{\partial^{2} \rho_{B}}{\partial x^{2}}+g \frac{\partial \rho_{B}}{\partial x}-\beta\left(\frac{\partial v_{g}}{\partial x}-\frac{\partial v_{s}}{\partial x}\right) \\
& \text { Wave Propagation }=\quad \text { Source } \quad-\quad \text { Drag } \\
& \text { (Zero for Developed Flow) }
\end{aligned}
$$

Equation (17) shows that the density wave propagates with the pseudo-wave velocity $\mathrm{C}_{\mathrm{s}}$, that gravity acts as a source for wave formation and that drag dampens the waves, when the relative velocity is positive. Generalization of Equation (17) to two and three dimensions shows that there will be additional source terms due to gradients of $\left(\mathrm{v}_{\mathrm{g}}-\mathrm{v}_{\mathrm{s}}\right)$ in the multiple dimensions. These terms may give additional peaks in the spectral analysis of bulk density. For developed flow the axial variation of the relative velocity becomes zero in Equation (17). Further, if we neglect the solids pressure effect, the sonic velocity, Equation (17) simplifies to a diffusion equation

$$
\frac{\partial^{2} \rho_{B}}{\partial t^{2}}=g \frac{\partial \rho_{B}}{\partial x}
$$

The variation of bulk density with height, $\mathrm{x}$ is obtained from an additional momentum balance. For developed one dimensional flow the pressure drop is balanced approximately by the weight of the bed, expressed by

$$
\frac{\partial P}{\partial x}=g \rho_{B}
$$

The pressure drop itself is given by the Ergun equation which for small relative velocities is as follows. 


$$
-\frac{d P}{d x}=\frac{150 \mu_{g}}{\left(\phi d_{p}\right)^{2}} \frac{\varepsilon_{s}^{2}}{\varepsilon^{3}} U_{o}
$$

We now relate the pressure drop to relative bed expansion using the method of Thompson (1978) who obtained a formula for frequency similar to that derived here. Thompson did not use conventional fluidization equations, but constructed a flow model of his own. First we note that for an expanded bed, the conservation of particles balance is as follows

$$
\varepsilon_{s o} x_{o}=\varepsilon_{s} x
$$

where $\varepsilon_{\mathrm{so}}$ and $\mathrm{x}_{\mathrm{o}}$ are some initial bed volume fraction and height of bed filled with particles. Using calculus, Equation (21) gives the differential expression as

$$
\frac{d x}{x}=-\frac{d \varepsilon_{s}}{\varepsilon_{s}}
$$

We now estimate the relative differential change of pressure using Equation (20), as the volume fraction, $\varepsilon_{\mathrm{s}}$ changes during the passage of a wave for a constant superficial velocity $\mathrm{U}_{\mathrm{o}}$. By calculus only,

$$
\frac{d\left(\frac{d P}{d x}\right)}{\frac{d P}{d x}}=-\left[3 \varepsilon_{s} / \varepsilon+2\right] \frac{d \varepsilon_{s}}{\varepsilon_{s}}
$$

Using the differential particle balance, Equation (22), the differential change of pressure drop can then be expressed as follows

$$
\frac{d\left(\frac{d P}{d x}\right)}{d x}=-\left[3 \varepsilon_{s} / \varepsilon+2\right] \frac{\varepsilon_{s}\left(\frac{d P}{d x}\right)}{\varepsilon_{s o} x_{o}}
$$

The above expression allows an estimate of the density gradient to be made.

Differentiation of Eq. (19) and substitution into Eq. (18) gives

$$
\frac{\partial^{2} \rho_{B}}{\partial t^{2}}=\frac{\partial^{2} P}{\partial x^{2}}
$$

But

$$
\frac{\partial^{2} P}{\partial x^{2}} \approx \frac{\Delta\left(\frac{d P}{d x}\right)}{\Delta x}
$$

Using Eq. (19) and (24), Eq. (25) becomes an ordinary differential equation

$$
\frac{d^{2} \rho_{B}}{d t^{2}}+\left[\frac{\left(3 \varepsilon_{s} / \varepsilon+2\right) \varepsilon_{s} g}{\varepsilon_{s o} x_{o}}\right] \rho_{B}=0
$$

Equation (27) is the equation for the vibration of a spring of a unit mass with the brackets representing a spring constant. Treating the bracket as a constant, the solution is

$$
\rho_{B}=A \cos \omega t+B \sin \omega t
$$

where 
The frequency of oscillations, $f$ is

$$
\omega=\left(\frac{g}{x_{o}}\right)^{\frac{1}{2}}\left[\frac{\left(3 \varepsilon_{s} / \varepsilon+2\right) \varepsilon_{s}}{\varepsilon_{s o}}\right]^{\frac{1}{2}}
$$

$$
f=\frac{\omega}{2 \pi}
$$

We see that the basic frequency is that caused by gravity, $\left(g / x_{o}\right)^{\frac{1}{2}}$ and that the frequency becomes very small as the volume fraction of particles becomes small. Verloop and Heertjes (1974) were the first to derive a frequency relation that has the basic term $\left(g / x_{o}\right)^{1 / 2}$ by treating a fluidized bed as a vibrating column. However their relation does not have the correct compressibility term achieved by using some key steps contained in Thompson's (1978) analyses. In Equation (27) the "elasticity" per unit mass is proportional to the volume fraction of solid. A denser bed has a greater elasticity.

\section{Comparison of Dominant Frequency Formula to Experiments}

Fig. 11 shows a comparison of the expanded bed dominant frequency, Equations (29) and (30), to the experimental data. Very low frequencies predicted by Equation (29) were also obtained by Sun and Gidaspow (1999) in the simulation of the PSRI riser in the challenge problem presented at the Eighth International Fluidization Conference in France. Fig. 11 and Equation (29) also suggest that for very dilute systems the frequency will vanish giving a steady state. This is the situation observed numerically. In such a situation the frequency jumps to a value of the order of 100 Hertz (Plasynski, et al ; 1993).

In Figure 11 the data obtained on the first floor is in the inlet section of the riser. There is more than one peak, as suggested by Equation (17). Gradients of velocity produce additional peaks in the bulk density. We have seen such a behavior earlier at IIT in bubbling beds (Seo, 1985; Gidaspow; et al,1989; Gidaspow,1994 Fig. 10.5). We have measured porosity with the gamma ray densitometer described here and pressure with a transducer. Fig. 12 shows several peaks near the inlet of a two dimensional bed but only one distinct peak in the region of bubble formation. Fig. 13 shows that the dominant peak obtained from pressure measurements, $f_{p}$, is the same as that due to the porosity measurement, $f_{\varepsilon}$, expect in the entrance region, for $\mathrm{h}<12 \mathrm{~cm}$. This follows from Equation (19), which is valid in the developed region. This equation also shows that if the density varies as the sine wave, then the pressure will be a cosine wave. Since $\cos (\omega t)=\sin \left(\omega t+\frac{\pi}{2}\right)$, there is the observed displacement of the two waves. Note also that the pressure waves (Gidaspow, 1994) move with the velocity $\left(\frac{\rho_{g}}{\rho_{s}}\right)^{\frac{1}{2}} \cdot C_{g} /\left(\varepsilon \varepsilon_{s}\right)^{\frac{1}{2}}$, where $\mathrm{C}_{\mathrm{g}}$ is the sonic velocity of the gas when $\rho_{s} \varepsilon_{s} \gg \varepsilon \rho_{g}$. At atmospheric pressure this velocity is of the order of $10 \mathrm{~m} / \mathrm{sec}$, considerably higher than $\mathrm{C}_{\mathrm{s}}$. Since in the developed region the dominant frequencies are the same for pressure and porosity, we 
can compare our experimental frequency equation to early bubbling bed data of Geldart and Baeyens (1974) who obtained dominant pressure peaks for several bed diameters. A comparison of the expanded bed equation to IIT and Geldart data is shown in Fig. 14. There is excellent agreement between the simplified theory and the data.

\section{Two Dimensional Oscillations: Gravity Waves}

The classical analysis of gravity waves (Elmore and Heald, 1969) can be used to understand two dimensional effects observed in fluidized beds. This analysis begins with the transient Bernoulli's equation made at the top of the bed. With a constant pressure and negligible velocity head, this equation reduces itself to

$$
g \Delta x=\frac{\partial \Phi}{\partial t}+\text { constant } \quad \text { at } \quad \mathrm{x}=\mathrm{h}
$$

where $\Phi$ is the velocity potential used by Davidson (1961) in his classical bubble model. Such a potential exists in the absence of voidage gradients, as shown in Gidaspow's book (1994). This is a severe restriction. Nevertheless, it yields some useful approximate solutions. Thus for irrotational flow,

$$
\nabla^{2} \Phi=0
$$

and

$$
\vec{v}=-\nabla \Phi
$$

Then in the direction of flow, $x$, Equations (31) and (33) give the balance

$$
g \frac{\partial \Phi}{\partial x}=\frac{\partial^{2} \Phi}{\partial t^{2}} \quad \text { at } \quad x=h
$$

At $\mathrm{x}=0$, the velocity is zero.

The solution to Equation (32) is obtained by separation of variables

$$
\Phi=X(x) Y(y) T(t)
$$

to be

$$
\frac{d^{2} T}{d t^{2}}+(g K \tanh K h) T=0
$$

where $\mathrm{K}$ is the separation constant having units of reciprocal length.

Equation (36) shows that the angular frequency $\omega$ is

$$
\omega=(g K \tanh K h) \frac{1}{2}
$$

Compare this to the compressible one dimensional case, Eq. (29).

The wave velocity, $\mathrm{C}$ is

$$
C=\frac{\omega}{K}=\left(\frac{g}{K} \tanh K h\right)^{\frac{1}{2}}
$$

For $K h<<1, \tanh K h \approx K h$ and Equation (38) gives

$$
C=(g h)^{\frac{1}{2}}
$$

This is the speed acquired by a particle falling a distance $h / 2$ from rest.

The second limit for $K h>>1$ or $h>>\frac{1}{K}$ gives tanh $K h=1$. Then 


$$
C=\left(\frac{g}{K}\right)^{\frac{1}{2}}=\left(\frac{\lambda g}{2 \pi}\right)^{\frac{1}{2}}
$$

where $\lambda$ is the wavelength. When we take $\lambda$ to be the bed diameter

$$
C=\left(\frac{D g}{2 \pi}\right)^{\frac{1}{2}}=0.3989(g D)^{\frac{1}{2}}
$$

versus the experimental slug velocity (Hovmand and Davidson, 1971)

$$
C_{\text {slug }}=0.35(g D)^{\frac{1}{2}} \text {. }
$$

\section{Reduction to Wave Equation}

Further analysis of fluidization oscillations following gravity wave theory is not very useful due to the assumption of incompressibility of bulk density made in the previous section. However, the one dimensional compressible analysis can be easily extended to more than one dimension by again neglecting $\mathrm{v}_{\mathrm{s}}$ compared with $\mathrm{C}_{\mathrm{s}}$ and assuming developed flow. The generalization of Equation (17) with the approximation made in the one dimensional analysis gives a wave equation with a source.

$$
\frac{\partial^{2} \rho_{B}}{\partial t^{2}}=C_{s}^{2} \nabla^{2} \rho_{B}+\left[\frac{\left(3 \varepsilon_{s} / \varepsilon+2\right) \varepsilon_{s}^{2} g \rho_{s}}{\varepsilon_{s o} x_{o}}\right]
$$

The solution to this equation is the approximate source solution given by Equation (28) plus the solution to the wave equation with the source in the brackets set to zero. The solution of the homogeneous wave equation, when put into the canonical form, has the form

$$
\rho_{\mathrm{B}, \text { homogeneos }}=f_{1}\left(x-C_{s} t\right)+f_{2}\left(x+C_{s} t\right)
$$

which shows that the waves move with the pseudo-sonic velocity $\mathrm{C}_{\mathrm{s}}$ in opposite directions. This is the theory behind the empirical correlation

$$
f_{C F B}=\frac{C_{s}-v_{s}}{L_{\text {emirical }}}
$$

shown in Figure 15. The pseudo-sonic velocity $\mathrm{C}_{\mathrm{s}}$ was taken relative to the solids velocity, as shown by the exact characteristics analysis.

The classical theory of wave propagation can be applied to this problem in the direction perpendicular to flow. Equation (13) shows that at the walls, $y=0$ and $y=L$ the gradient of bulk density is zero. Then separation of variables of the homogeneous portion of the wave equation is as follows. Let

$$
\rho_{B}=T(t) Y(y)
$$

The ordinary differential equations become as follows, where prime denotes the derivative

$$
\begin{aligned}
& \frac{T^{\prime \prime}}{T}=C_{s}^{2} \frac{Y^{\prime \prime}}{Y}=-\omega^{2} \\
& T^{\prime \prime}+\omega^{2} T=0 \text { and } Y^{\prime \prime}+\frac{\omega^{2}}{C_{s}^{2}} Y=0
\end{aligned}
$$


with the boundary conditions

$$
Y^{\prime}(0)=Y^{\prime}(L)=0
$$

which give

$$
Y=A \cos \left(\frac{\omega_{n}}{C_{s}}\right) y
$$

and the eigenvalues

$$
\frac{\omega_{n} L}{C_{s}}=\pi n, n=1,2,3, \ldots
$$

Hence with the zero gradient boundary conditions the fundamental frequency is:

$$
\omega_{1}=\frac{\pi C_{s}}{L}
$$

or

$$
\omega_{1}=\pi f_{C F B}
$$

Figure 15 gives a length $L=\pi L_{\text {empirica }}$ of about 6 meters which is the length of the CFB. Note that the boundary conditions at the top and bottom of the bed cannot be given exactly in terms of bulk density only. Hence the length in the direction of flow is not precisely known for wave propagation. This analysis, however, greatly aids in interpreting the numerical solution of the complete equations of motion.

\section{Non Dissipating System at Resonant Frequency}

A most important characteristic of fluidized bed is that they never reach a true steady state. In dense beds this unsteadiness is visually observed as bubbles that form near the bottom of the bed, rise and then erupt at the surface, just like boiling water in a pot. In risers the unsteadiness is visually observed and computed (Tsuo and Gidapow, 1989; Sun and Gidaspow, 1999) as clusters of particles that form at the top, descend to the bottom of the bed and are transported back up in the center of the pipe. If the intensity of oscillations is not strong, as in dilute riser flow, or if the restitution coefficient is made too small, the computer code gives a steady state which may or may not represent the correct time averaged behavior. Hence an approximate analytical behavior needs to be investigated. Classical wave theory aids in the understanding.

The Navier-Stokes equations for compressible fluids can be decomposed into a wave equation for a potential with damping due to the fluid viscosity and into a stream function equation (Tolstoy, 1973; Morse and Feshbach, 1953). For zero viscosity that is for a high Reynolds number, the result is an undamped wave equation for a potential which by substitution

$$
\Phi(x, y, z) e^{-i \omega t}
$$

can be transformed into the classical Helmholtz equation for the eigenfunction $\Phi_{n}$

$$
\nabla^{2} \Phi_{n}+k_{n}^{2} \Phi_{n}=0
$$

where

$$
k_{n}=\omega_{n} / C_{s}
$$

The corresponding equation for a unit source solution, the Green's function $G_{k}$ is

$$
\nabla^{2} G_{k}+k_{\text {source }}^{2}=-4 \pi \delta\left(\vec{r}-\vec{r}_{\text {source }}\right)
$$

A standard Green's function construction (Morse and Feshbach, 1953) gives 


$$
G_{k}\left(\vec{r} ; \vec{r}_{\text {source }}\right)=4 \pi \sum_{n=1}^{\infty} \frac{\Phi_{n}(\vec{r}) \Phi_{n}\left(\vec{r}_{\text {source }}\right)}{k_{n}^{2}-k^{2} \text { source }}
$$

In Equation (54) when

$$
k_{n}=k_{\text {source }}
$$

we have a singularity. These are the infinities when a non dissipative vibrating system is driven at one of its resonant frequencies. For fluidization, the porosity goes to infinity.

\section{Resonant Height}

The brief review of resonance for a non dissipative system shows that at resonance we equate the frequency of the source to the natural frequency of the system, as given by Equation (55). For the fluidized bed we equate the frequency given by Equations (51) and (44) to the gravity wave frequency, Equation (29). For the fundamental frequency, $\omega$, with $C_{S}$ expressed relative to the velocity of the solids, the result is

$$
\frac{C_{s} \pi}{L}=\left(\frac{g}{L}\right)^{\frac{1}{2}}\left[\frac{\left(3 \varepsilon_{s} / \varepsilon+2\right) \varepsilon_{s}}{\varepsilon_{s o}}\right]^{\frac{1}{2}}
$$

The resonant length, $\mathrm{L}_{\text {resonant }}$, neglecting second order terms in volume fractions, gives the simple result

$$
L_{\text {resonant }}=\frac{\pi^{2} C_{s}^{2}}{2 g \varepsilon_{s} / \varepsilon_{s o}}
$$

To make a quick estimate, it is best to express the pseudo-sonic velocity, $\mathrm{C}_{\mathrm{s}}$ in terms of the granular temperature, $\theta$ using the ideal equation of state for particles (Gidaspow and Huilin, 1998), $P_{s}=\varepsilon_{s} \rho_{s} \theta$ which is valid near 5\% solids or less.

$$
C_{s}^{2}=\theta
$$

Then the resonant length becomes

$$
L_{\text {resonant }}^{\text {dilute }}=\frac{\pi^{2} \theta}{2 g \varepsilon_{s} / \varepsilon_{s o}}
$$

For dense bubbling beds the collisional pressure $P_{s}=4 \varepsilon_{s}^{2} \rho_{s} g_{o} \theta$ can be used where $\mathrm{g}_{0}$ is the radial distribution function at contact. The result is

$$
L_{\text {resonant }}^{\text {dense }}=\frac{4 \pi^{2} g_{o} \theta}{g / \varepsilon_{\text {so }}}
$$

For bubbling beds Cody's data (Cody and Buyevich, 1998) gives a granular temperature of $10(\mathrm{~cm} / \mathrm{s})^{2}$. With $\mathrm{g}_{0}=5$, Equation (60) shows that resonance occurs at a length of $2 \mathrm{~cm}$. Resonance may be an alternative way to interpret bubble formation.

For the Synthol reactor discussed in the introduction, Equation (59) gives a length of about 10 meters. Hence a smaller pilot plant had no violent vibrations, while the commercial unit encountered resonance. 


\section{An Analytical Solution For Granular Temperature}

Values of granular temperature vary by at least four order of magnitude, from about $1(\mathrm{~m} / \mathrm{s})^{2}$ in a riser for FCC particles to less than $1(\mathrm{~cm} / \mathrm{s})^{2}$ for $45 \mu m$ catalyst in a liquid-solid fluidized bed (Gidaspow and Huilin, 1998b). Cody and Goldfarb's (1998) values of granular temperature in a bubbling bed at a ratio of fluid velocity to the velocity of minimum fluidization of two are about $15(\mathrm{~cm} / \mathrm{s})^{2}$ for a $50 \mu \mathrm{m}$ size particle, rise to 35 $(\mathrm{cm} / \mathrm{s})^{2}$ for about $90 \mu m$ particle and then decrease to $5(\mathrm{~cm} / \mathrm{s})^{2}$ for a $150 \mu m$ size and then finally increase with particle size. Such a behavior is known to exist for heat transfer coefficients in bubbling beds and has never been explained successfully. Hence we only present an analysis that explains the order of magnitude difference between various measurements, since a full explanation in not yet available.

In developed flow in a riser with flow of elastic particles, the granular temperature balance (Gidaspow, 1994) involves a balance between conduction and generation. In cylindrical coordinates it is as follows for a constant conductivity, $\kappa$ and particle viscosity, $\mu_{s}$.

$$
\frac{\kappa}{r} \frac{d}{d r}\left(r \frac{d \theta}{d r}\right)=-\mu_{s}\left(\frac{\partial u_{s}}{\partial y}\right)^{2}
$$

As a limit we had assumed that all dissipation occurs at the wall. We prescribe the wall granular temperature at pipe surface; $r=R$

$$
\theta(R)=\theta_{w}
$$

The solids velocity, $u_{s}$ can be approximated as the difference between the gas and the terminal velocity leading to a lower shear rate for larger particles and a possible explanation for the maximum in Cody and Goldfarb's data. However, here we assume homogeneous flow, as is roughly correct for flow of FCC particles in the CFB.

We had estimated the FCC viscosity (Miller and Gidaspow, 1992) from the mixture momentum balance,

$$
\frac{\mu_{s}}{r} \frac{d}{d r}\left(r \frac{d u_{m}}{d r}\right)=\frac{d P}{d z}-\rho_{m} g
$$

and the measurement of the solids radial velocity profile. For small slip the mixture velocity $u_{m}$ and the solids velocity $u_{s}$ are nearly equal. Then Equation (63) can be used to obtain the velocity gradient in Equation (61). Unfortunately the mixture density is a function of the radius $r$. One can, however, divide the flow into two regions, the core and the dense annulus. Neglecting the thin annulus downflow region, integration of Equation (63) then gives the usual Poiseuille flow. Integration of Equation (61) then gives a fourth-power dependence of granular temperature on radius, like the thermal temperature rise in Poiseuille flow (Schlichting, 1960). In terms of the mean velocity $\bar{u}$, the relation between the maximum granular temperature, $\theta_{\max }$ and $\bar{u}$ then is the same as the relation between the thermal temperature and the mean velocity.

$$
\theta_{\max }-\theta_{w}=\left(\frac{\mu_{s}}{\kappa}\right) \bar{u}^{2}
$$

Equation (64) shows that the granular temperature is of the order of the fluid velocity squared. Figure 10 fully supports this analysis. Equation (64) shows the reasonable result 
that at low fluid velocities, the granular temperature will be correspondingly low. It provides a first order estimate of the granular temperature and the pseudo-sonic velocity needed in the present analysis.

\section{Vortices Due to Standing Waves}

Joshi and Sharma (1979) solved a stream function equation with vorticity for bubble column and pointed out the possibility of multiple circulation cells. Wu and Gidaspow (1999) solved the separate phase conservation of mass, momentum, species and energy equations for a slurry bubble column reactor using granular flow kinetic theory and observed multiple circulation cells in addition to the basic upflow in the center and downflow at the walls. The understanding and modification of flow patterns by, for example, proper placement of heat exchangers into the column is needed for an improved reactor design. The origin of the multiple circulation cells may be in the vortices formed by the stationary porosity waves.

The classical Rayleigh kundt's tube solutions, depicted in Fig. 16, can be applied to fluidization by replacing the sonic velocity with pseudo-sonic velocity, $C_{s}$. Tolstoy (1973) shows how such solutions are obtained starting with the Navier-Stokes' equations. The standing waves are the solutions that depend on the position only.

Outside the boundary layer, there exists a velocity in the $\mathrm{x}$ direction given by

$$
V_{x}=\frac{3 V_{o}^{2}}{8 C_{s}} \sin 2 \alpha x
$$

where

$$
\alpha=\frac{\omega}{C_{s}}
$$

We see that the periodic solution (65) depends upon the value of the prescribed frequency, which is the gravity wave frequency derived earlier; and on the pseudo-sonic velocity, $C_{s}$. Figure 17 gives a representation of the flow patterns for some typical values for the riser and for a denser bed. The riser flow pattern is similar to that measured and computed using the complete set of Navier-Stokes' equations with granular flow theory (Neri and Gidaspow, 1999). Equation (66), however, permits a quick estimate of length using the prescribed forced frequency, $\omega$, and the pseudo-sonic velocity, $C_{s}$. Such patterns may also be achieved by vibrations, where $\omega$ can be varied independently.

\section{Nomenclature}

$\mathrm{e}$

$f$

$\mathrm{g}$

$\mathrm{g}_{\mathrm{o}}$

$\mathrm{h}$

$\mathrm{k}$

$\mathrm{k}_{\mathrm{n}}$

$\mathrm{r}$

$\vec{r}$
Restitution coefficient

Frequency of oscillation

Gravitational acceleration

Radial distribution function

Bed height

Particles thermal conductivity

Wave number

Radial coordinate

Space vector 


\section{$\mathrm{t}$}

$\mathrm{V}$

$<v>$

$\mathrm{x}$

$\mathrm{y}$

$\mathrm{z}$

C

$\mathrm{C}_{\mathrm{s}}$

$\mathrm{C}_{\mathrm{p}}$

D

F

$\mathrm{G}_{\mathrm{k}}$

K

L

$\mathrm{P}$

$\mathrm{R}$

$\operatorname{Re}$

$\mathrm{U}_{\mathrm{o}}$

\section{Greek Letters}

$\alpha$

$\beta$

$\delta$

$\varepsilon$

$\theta$

$\kappa$

$\lambda$

$\mu$

$\rho$

$\phi$

$\omega$

$\Phi$

\section{Subscripts}

$\mathrm{g}$

$\mathrm{m}$

o

$\mathrm{p}$

$\mathrm{p}$

$\mathrm{s}$

W

B

$\varepsilon$
Time

Velocity

Average fluctuating velocity of particles

Direction of flow

Perpendicular direction to flow

Axial coordinate

Wave velocity

Pseudo-sonic velocity of particles

Heat capacity of particles

Pipe diameter

Flux

Greens' function

Separation constant

Bed diameter

Pressure

Pipe diameter

Reynolds number

Inlet superficial gas velocity

Wave number

Drag coefficient

Dirac delta function

Volume fraction

Granular temperature

Granular temperature conductivity

Wavelength

Viscosity

Density

Sphericity

Angular frequency

Velocity potential

Gas

Mixture

Initial value

Due to pressure

Particle

Solid

Wall property

Bulk

Due to porosity 


\section{Acknowledgment}

This study was supported by the National Science Foundation Fluid, Particulate and Hydraulic System Grant No. CTS-96-10053, by a Dow Corning Corporation gift and by Department of Energy Grant No. DE-FG-98FT40117.

\section{References}

Anderson K., Sundaresan S. and Jackson R. 1995, Instabilities and the formation of bubbles in fluidized beds, J. Fluid Mech., 303, 327-366.

Anderson T. B. and Jackson R. 1969, A fluid mechanical description of fluidized beds, comparison of theory and experiment, I \& EC Fundamentals, 8, 137-144.

Baeynes J. and Geldart D. 1974, An investigation into slugging fluidized beds, Chem. Eng. Science, 29, 255-265.

Bagnold R. A. 1954, Experiments on a gravity free dispersion of large solid spheres in a Newtonian fluid under shear, Proc. Roy. Soc., A255, 49-63.

Baumgarten P. K. and Pigford R. L. 1960, Density fluctuations in fluidized beds, AIChE $J ., 6,115-121$.

Benyahia S., Arastoopour H. and Knowlton T.M. 1998, Prediction of solids and gas flow behavior in a riser using a computational multiphase flow approach, Proc. 9 th Eng. Found. Conf. Fluidization, Durango, Colorado.

Bouillard J. X. and Miller A. 1994, Experimental investigations of chaotic hydrodynamic attractors in circulating fluidized bed, 4th Int. Conf. on CFB, Engineering Fundation, 80-85.

Buyevich Y. A. and Cody G.D. 1998, Particle fluctuations in homogeneous fluidized beds, Prep. for Brighton World Congress on Particle Tech., 3, Brighton, UK, Paper 207.

Campbell C. S. and Wang D. G. 1991, Particle pressure in gas fluidized beds, J. Fluid Mech., 227, 495-508.

Carlos C. R. and Richardson J. F. 1986, Solid movement in liquid fluidzed beds I: particle velocity distribution, Chem. Eng. Sci., 23, 813-824.

Chen J. C., Polashenski W. and Tuzla K. 1994, Normal solid stress in fluidized beds, AIChE Annular Meeting, San Francisco, 14-19.

Cody G. D. and Goldfarb D. J. 1998, Bifurcation in particle granular temperature of monodispersed glass spheres at the Geldart A/B transition, Fluidization IX, edited by L. S. Fan and T. M. Knowlton, New York: Engineering Foundation, 53-60.

Culik F. E. C. 1994, Some recent results for nonlinear acoustics in combustion chambers, AIAA J., 32, 146-169.

Davidson J. F. 1961, Symposium on fluidization: discussion, Trans. Inst. Chem. Engrs., 39, 230-232

Ding J. and Gidaspow D. 1990, A bubbling fluidization model using kinetic theory of granular flow, AIChE J. 36, 523-538.

Elmore W.C. and Heald M.A. 1969, Physics of Waves, New York, Mc Graw-Hill. 1969.

Gidaspow D. and Huilin L., 1996, Collisional viscosity of FCC particles in a CFB, AIChE. J. 42, 2503-2510.

Gidaspow D. and Huilin L., 1998a, Equation of state and radial distribution function of FCC particles in a CFB, AIChE. J. 44, 279-293. 
Gidaspow D. and Huilin L., 1998b, A comparison of gas-solid and liquid-solid fluidization using kinetic theory and statistical mechanics, Fluidization IX, edited by L. S. Fan and T. M. Knowlton, New York: Engineering Foundation, 662-668.

Gidaspow D. 1994, Multiphase flow and fluidization: Continuum and kinetic theory descriptions, Boston: Academic Press Inc.

Gidaspow D., Gamwo I., Tsuo Y. P. and Ding J. 1989, Two dimensional fluidized bed experiments, report to Argonne National Laboratory Cont. No. 82312401.

Hovmand S. and Davidson J. F. 1971, Pilot plant and laboratory scale fluidized reactors at high gas velocities; the relevance of slug flow, Fluidization, edited by J. F. Davidson and D. Harrison, Academic Press, 193-257.

Jackson R. 1985, Hydrodynamic stability of fluid-particle systems, in Fludization, edited by J. F. Davidson, R. Clift and D. Harrison, Academic press, 47-72.

Jackson R. 1993, Progress toward a mechanics of dense suspensions of solid particles, AIChE Symp. Ser., 90, 1-14.

Jin Y., Zhu J.X. and Yu Z.Q. 1997, Novel configurations and variants, in Circulating Fluidized Beds, edited by J.R. Grace, A.A. Avidan and T.M. Knowlton, Blackie Academic \& Professionals, London, 525-567.

Johnson P. C. and Jackson R., 1987 Frictional-Collisional Constitutive Relations for Granular Materials with Application to Plane Shearing, J. Fluid Mech., 176, 67-93.

Joshi J. B. and Sharma M. M. 1979, A circulation cell model for bubble columns, Trans. I. Chem. E., 57, 244-251.

Kuippers J. A. M., Hoomans B. P. B. and van Swaaij W. P. M. 1998, Hydrodynamic models of gas-fluidized beds and their role for design and operation of fluidized bed reactors, Fluidization IX, edited by L. S. Fan and T. M. Knowlton, New York: Engineering Foundation, 15-30.

Lun C. K. K., Savage S. B., Jeffrey D. J. and Chepurniy N. 1984, Kinetic theories for granular flow: inelastic particles in Couette flow of singly inelastic particles in a general flow field, J. Fluid Mech., 140, 223-256.

Lyczkowsky R.W., Gamwo I.K., Dobran F., Ali H., Chao B.T., Chen M.M. and Gidaspow D. 1993, Validation of computed solids hydrodynamics and pressure oscillations in a bubbling atmospheric fluidized bed, Powder Technology, 76, 65.

Matsen J. M. 1997, Design and scale up of CFB catalytic reactors, in Circulating Fluidized Beds, edited by J. R. Grace, A. A. Avidan and T. M. Knowlton, Blackie Academic and Professionals, 498.

Miller A. and Gidaspow D. 1992, Dense, vertical gas-solid flow in a pipe, AIChE J., 11, 1801-1813.

Miller A. 1991, Dense vertical gas-solid flow in a pipe, Ph D. Diss., Illinois Institute of Technology, Chicago.

Morse P.M. and Feshbach H. 1953, Methods of Theoretical Physics, New York, Mc Graw-Hill.

Neri A. and Gidaspow D. 1999, Riser hydrodynamics: simulation using kinetic theory, submitted to AIChE J.

Neri A. 1998, Multiphase flow modeling and simulation of explosive volcanic eruptions, Ph.D. thesis, Illinois Institute of Technology, Chicago. 
Neri A., Gidaspow D., Macedonio G. and Barberi F. 1998, Simulation of explosive volcanic eruptions by using multiphase flow models, AIChE annual meeting preprints, Vol. II, Particte Technology Forum, Miami Beach, 527-533.

Ocone R. \& Astarita G. 1994, On waves of particulate phase pressure in granular materials, J. Rheol., 38, 129-139.

Plasynski S. I., Klinzing G. E. and Mathur M. P. 1993, Pressure fluctuations investigation for high pressure vertical pneumatic transport, 4th Int. Conf. on CFB, Engineering Foundation, 260-265.

Samuelsberg A. and Hjertager B.H. 1996a, An experimental and numerical study of flow patterns in a circulating fluidized bed reactor, Int. J. Multiphase Flow, 22, 575-591.

Samuelsberg A. and Hjertager B.H. 1996b, Computational modeling of gas particle flow in a riser, AIChE J., 42, 1536-1546.

Savage S. B. 1988, Streaming motions in a bed of vibrationally fluidized dry granular material, J. Fluid Mech., 194, 457-478.

Schlichting H. 1960, Boundary Layer Theory, Mc Graw Hill, New York.

Seo Y. C. 1985, Fluidization of single and binary size particles, Ph.D. thesis, Illinois Institute of Technology, Chicago.

Shingles T. and Mc Donald A. F. 1997, Commercial experience with Synthal CFB reactors, in Circulating Fluidized Beds, edited by J. R. Grace, A. A. Avidan and T. M. Knowlton, Blackie Academic and Professionals, 43.

Sinclair J. L. and Jackson R. 1989, Gas-solid flows in a vertical pipe with particle-particle interaction, AIChE J., 35, 1473-1486.

Sinclair J. L. 1997, Hydrodynamic modeling, in Circulating Fluidized Beds, edited by J. R. Grace, A. A. Avidan and T. M. Knowlton, Blackie Academic and Professionals, 149.

Squires A. M., Kwauk M. and Avidan A. A. 1985, Fluid beds: at last, challenging two entrenched practices, Science, 230, 1329-1337.

Sun B. and Gidaspow D. 1999, Computation of circulating fluidized bed riser flow for the Fluidization VIII benchmark test, Ind. Eng. Chem. Res., 38, 787-792.

Sun B. and Koves W.J. 1998, Application of numerical hydrodynamic model in FCC design, Particle Technology Forum, AIChE meeting, Miami Beach, 2, 469-476.

Therdthianwong A. and Gidaspow D. 1994, Hydrodynamics and $\mathrm{SO}_{2}$ sorption in a CFB loop, in Circulating Fluidized Bed Technology IV, edited by A.A. Avidan, proceedings of the AIChE 4 th International Conference on Circulating Fluidized Beds, New York, 551-558.

Thompson A. S. 1978, Instabilities in a coal burning fluidized bed, Oak Ridge

National Laboratory Report, Tennessee, ORNL/HUD/MIUS-3B.

Tolstoy I., 1973, Wave Propagation, New York, Mc Graw-Hill.

Tsinontides S. C. and Jackson R. 1993, The mechanics of gas fluidized beds with an interval of stable fluidization, J. Fluid Mech., 255, 237-274.

Tsuo Y.P. and Gidaspow D. 1990, Computation of flow patterns in circulating fluidized beds, AIChE J., 36, 885-896.

Verloop J. and Heertjes P. M. 1974, Periodic pressure fluctuations in fluidized beds, Chem. Eng. Science, 29, 1035-1042. 
Weimer A. W., Gyure D. C. and Clough D. E. 1985, Application of gamma-radiation density gauge for determining hydrodynamic properties of fluidized beds, Powder Technology, 2, 179-194.

Wu Y. and Gidaspow D. 1999, Hydrodynamic simulation of methanol synthesis in gasliquid slurry bubble column reactors, to appear in Chem. Eng. Science. 
Table A Summary of Experimental Data

\begin{tabular}{||l|l|l|l|l|l|l|l|l||}
\hline $\begin{array}{l}\mathrm{U}_{\mathrm{g}} \\
\mathrm{m} / \mathrm{s}\end{array}$ & $\begin{array}{l}\mathrm{W}_{\mathrm{s}} \\
\mathrm{kg} / \mathrm{m}^{2} \mathrm{~s}\end{array}$ & $\boldsymbol{\varepsilon}_{s}$ & $\begin{array}{l}\sigma_{z} \\
\mathrm{~cm} / \mathrm{s}\end{array}$ & $\begin{array}{l}\sigma_{r} \\
\mathrm{~cm} / \mathrm{s}\end{array}$ & $\begin{array}{l}\boldsymbol{\theta} \\
(\mathrm{m} / \mathrm{s})^{2}\end{array}$ & $\begin{array}{l}\mathrm{V}_{\mathrm{p}} \\
\mathrm{m} / \mathrm{s}\end{array}$ & $\begin{array}{l}\mathrm{P}_{\mathrm{S}} \\
\mathrm{P}_{\mathrm{a}}\end{array}$ & $\begin{array}{l}\text { Dominant } \\
\text { Frequency } \\
(\mathrm{Hz})\end{array}$ \\
\hline 2.67 & 34.1 & $\begin{array}{l}0.113 \\
6\end{array}$ & $\begin{array}{l}247.3 \\
5\end{array}$ & 20.36 & 2.067 & 2.31 & 430.07 & 0.1953 \\
\hline 2.67 & 27.8 & $\begin{array}{l}0.109 \\
8\end{array}$ & $\begin{array}{l}223.1 \\
9\end{array}$ & 18.74 & 1.684 & 2.03 & 424.63 & 0.1269 \\
\hline 2.67 & 18.3 & $\begin{array}{l}0.070 \\
9\end{array}$ & $\begin{array}{l}225.0 \\
4\end{array}$ & 14.28 & 1.824 & 1.68 & 174.94 & 0.0572 \\
\hline 2.14 & 27.8 & $\begin{array}{l}0.096 \\
5\end{array}$ & $\begin{array}{l}232.0 \\
3\end{array}$ & 19.13 & 1.819 & 1.74 & 290.85 & 0.0976 \\
\hline 2.14 & 21.3 & $\begin{array}{l}0.057 \\
2\end{array}$ & $\begin{array}{l}218.3 \\
2\end{array}$ & 14.05 & 1.602 & 1.69 & 154.25 & 0.0651 \\
\hline 1.61 & 27.8 & $\begin{array}{l}0.087 \\
9\end{array}$ & $\begin{array}{l}239.5 \\
7\end{array}$ & 18.06 & 1.935 & 1.34 & 277.76 & 0.0879 \\
\hline
\end{tabular}

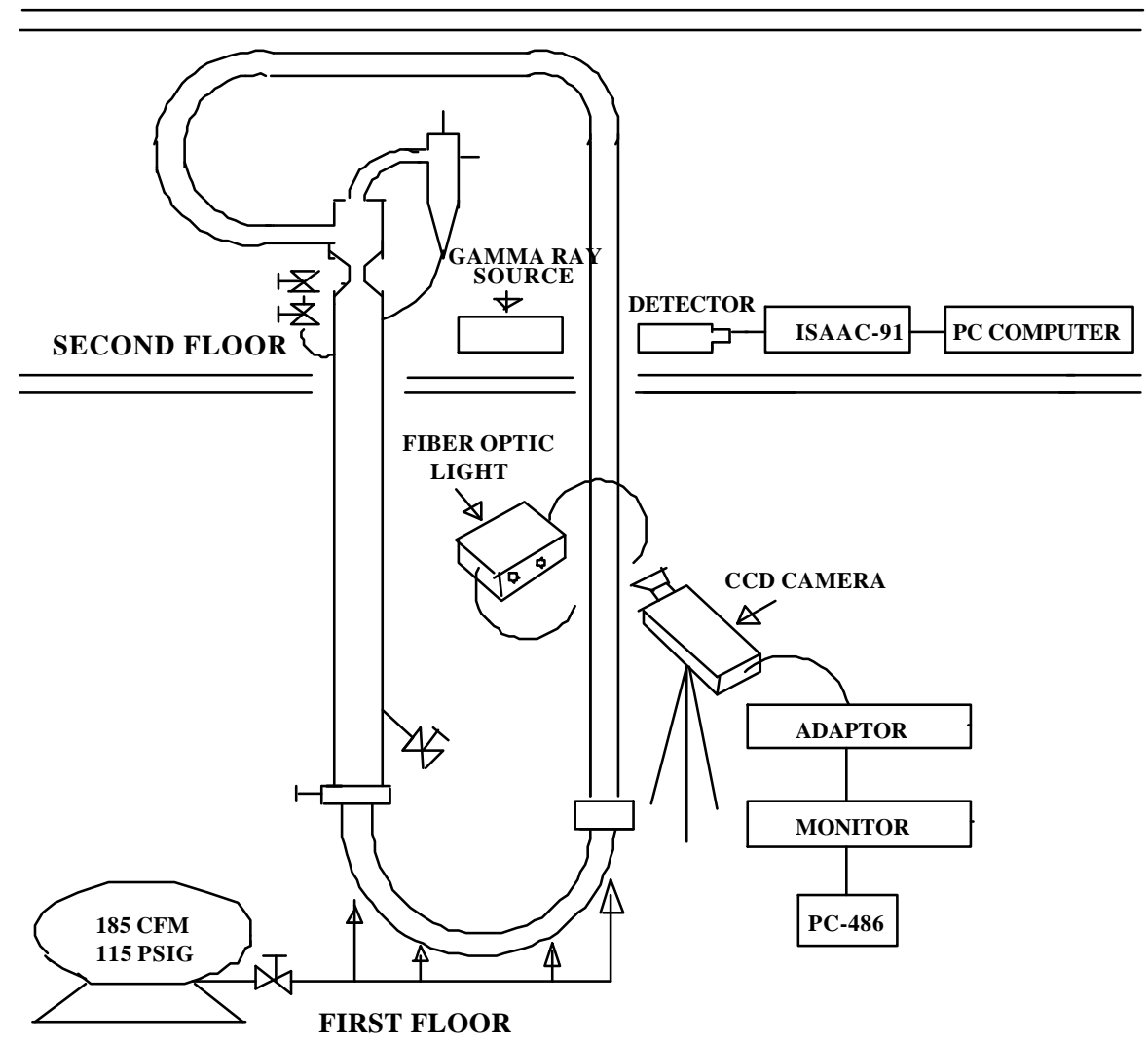

Fig. 1a IIT Circulating Fluidized Bed With Particle Pressure Transducer, CCD Camera and Gamma Ray Densitometer 


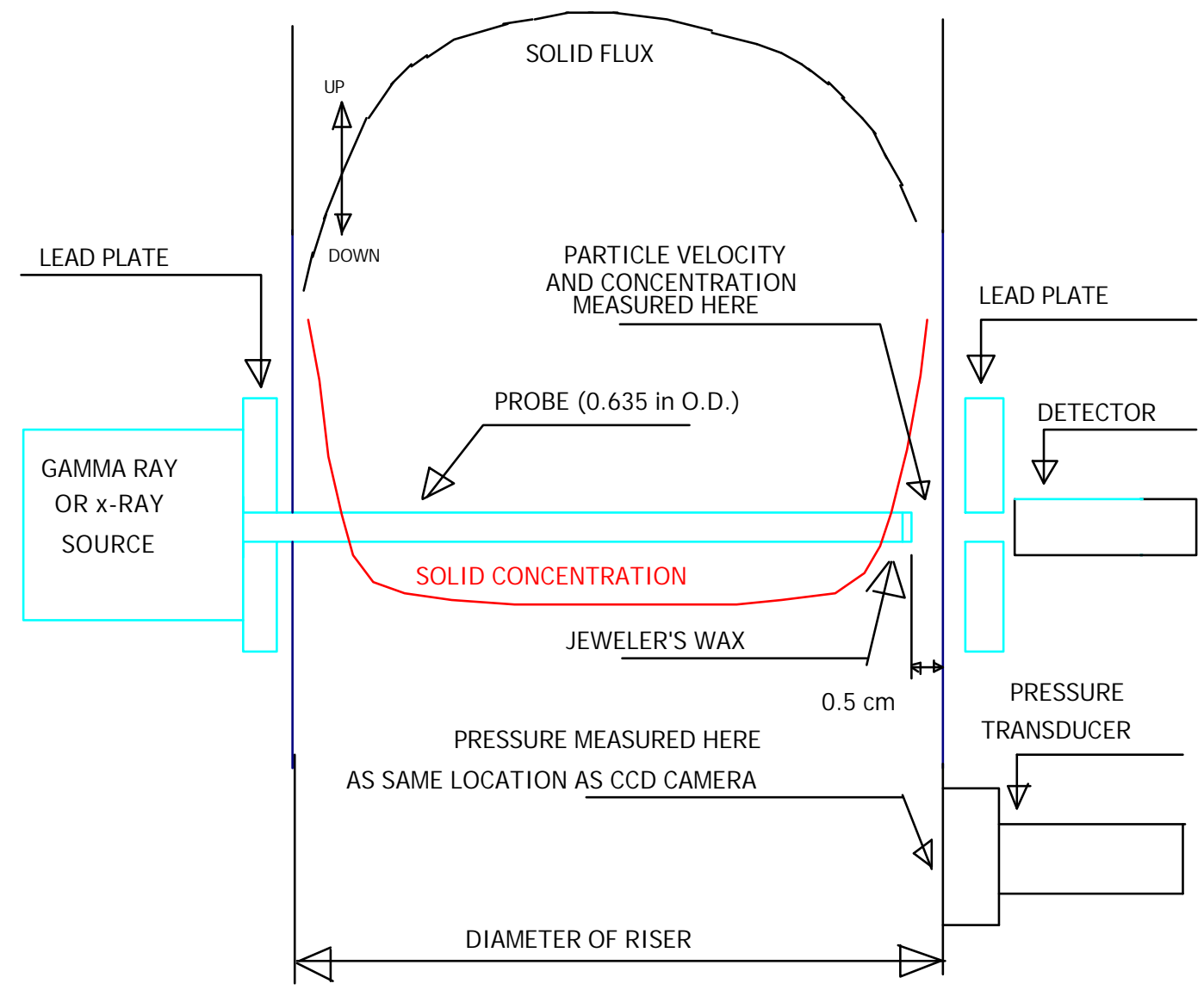

Fig . 1b Sketch of Determination of Particle Concentrations in This Study ( Miller and Gidaspow , 1992) 


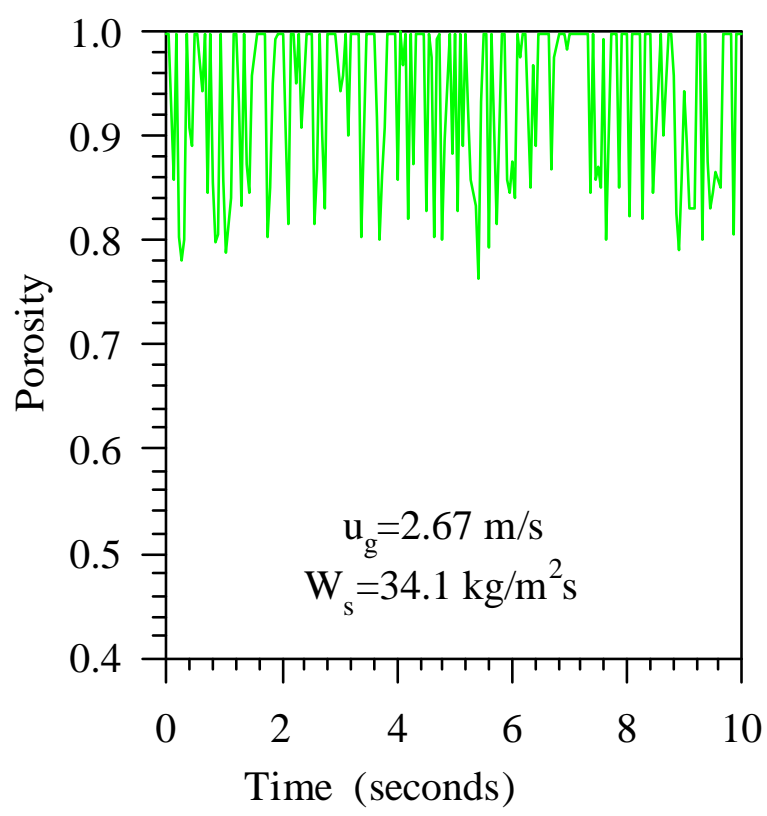

Fig. 2 Experimental Instantaneous Porosity

Fluctuations in The Dilute Region

(Second Floor, Fig. 1a)

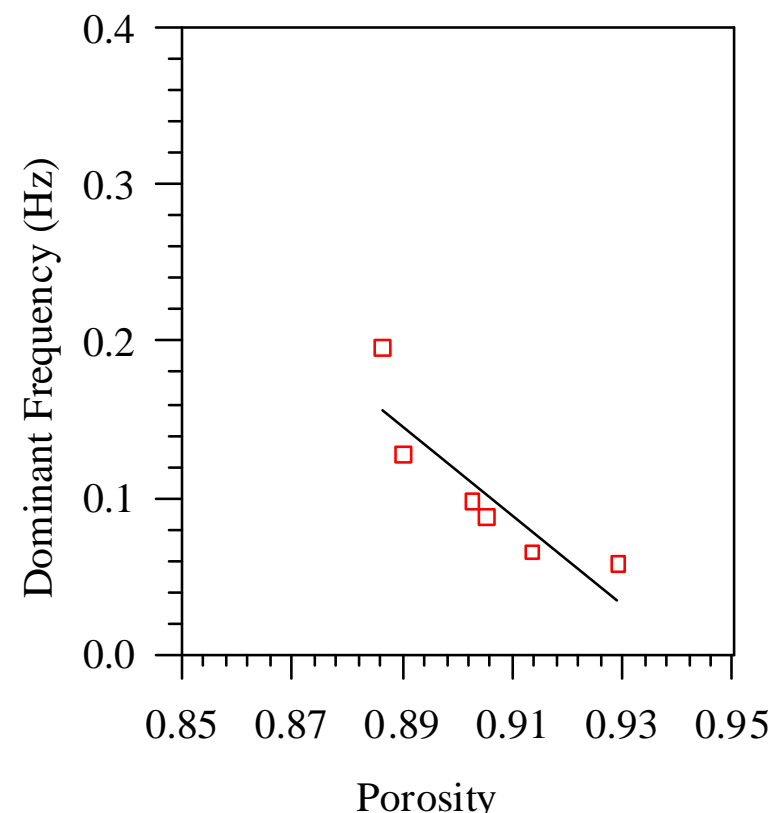

Fig. 5 Dominant Frequency in the Dilute Phase Region

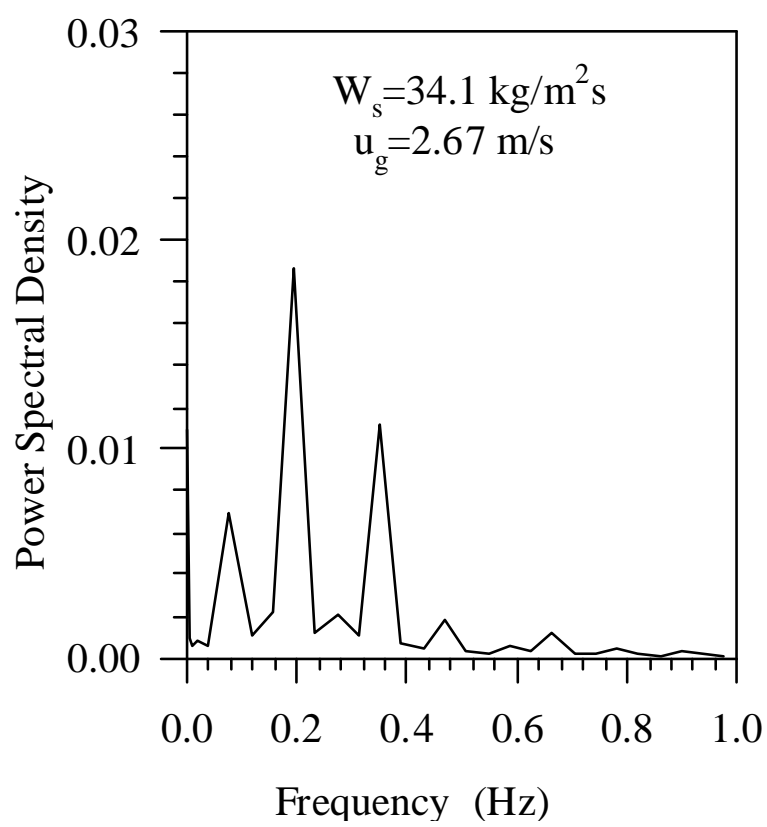

Fig. 3 Power Spectrum Density of Porosity Fluctuations in The Dilute Region

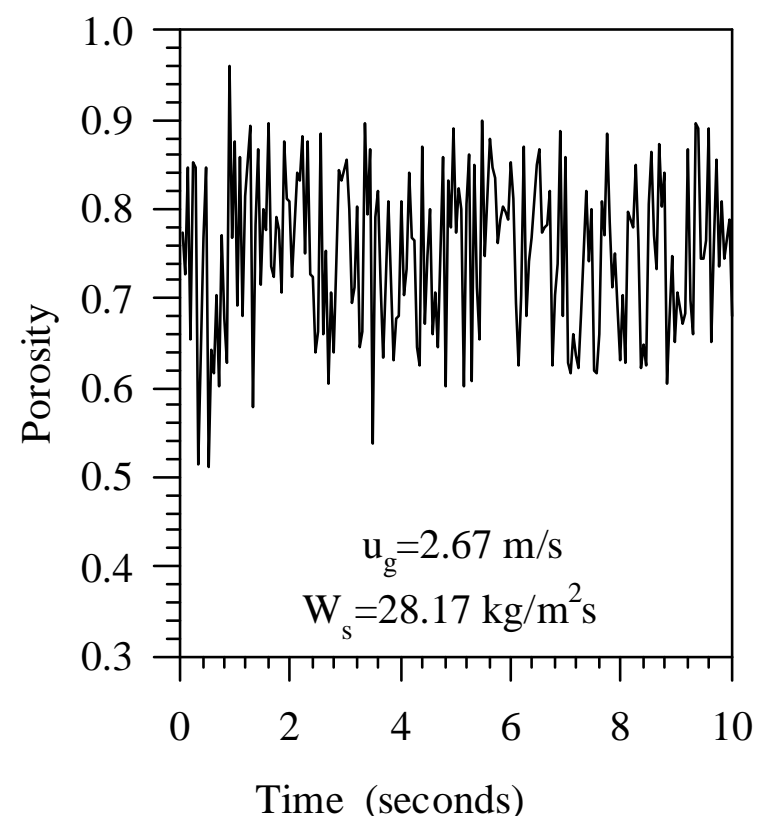

Fig. 6 Experimental Instantaneous Porosity

Fluctuations in the Dense Regime $\left(\mathrm{U}_{\mathrm{g}}=2.67 \mathrm{~m} / \mathrm{s}, \mathrm{W}_{\mathrm{s}}=28.17 \mathrm{~kg} / \mathrm{m}^{2} \mathrm{~s}\right.$; First Floor, Fig. $\left.1_{\mathrm{a}}\right)$ 


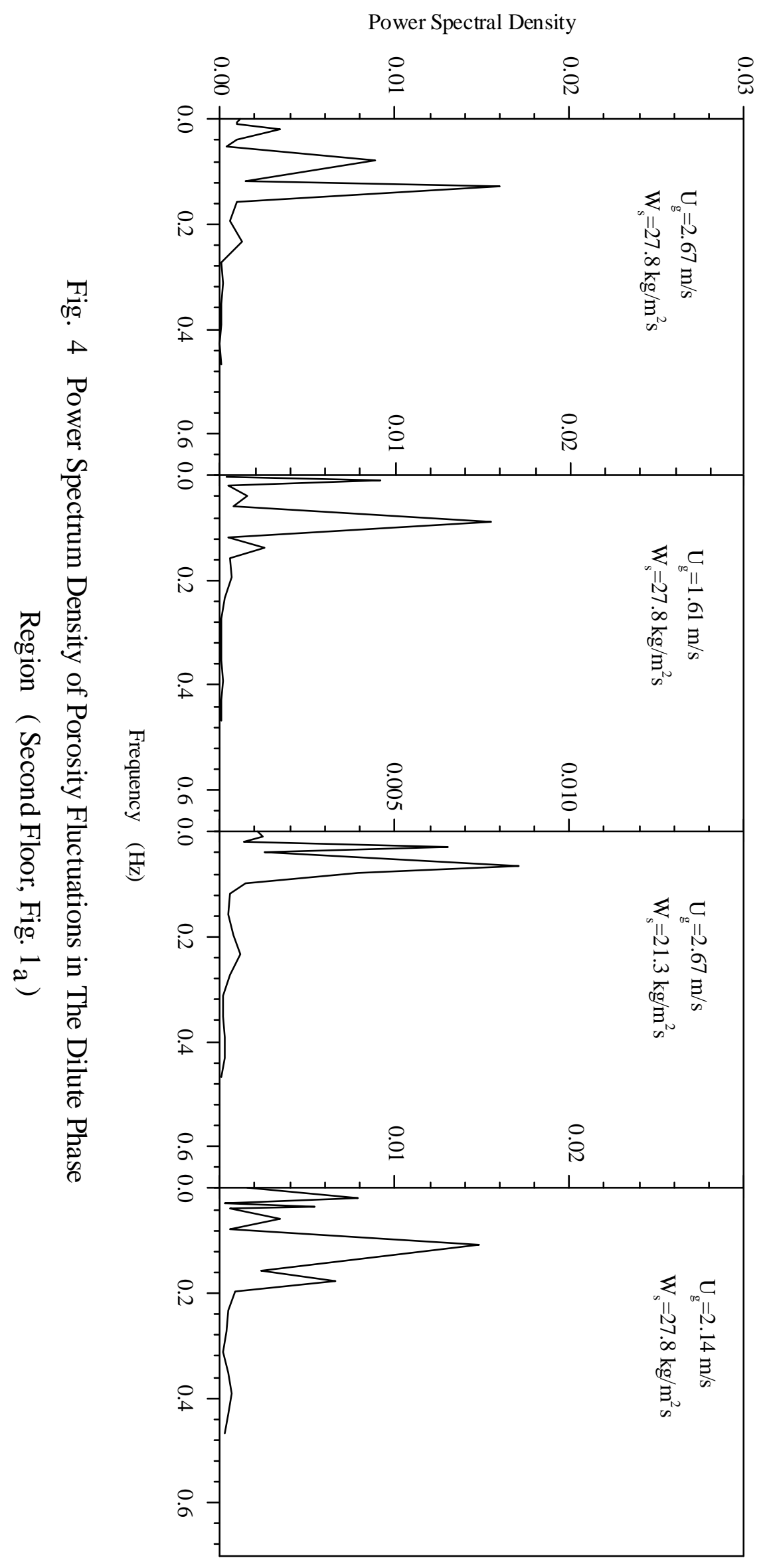




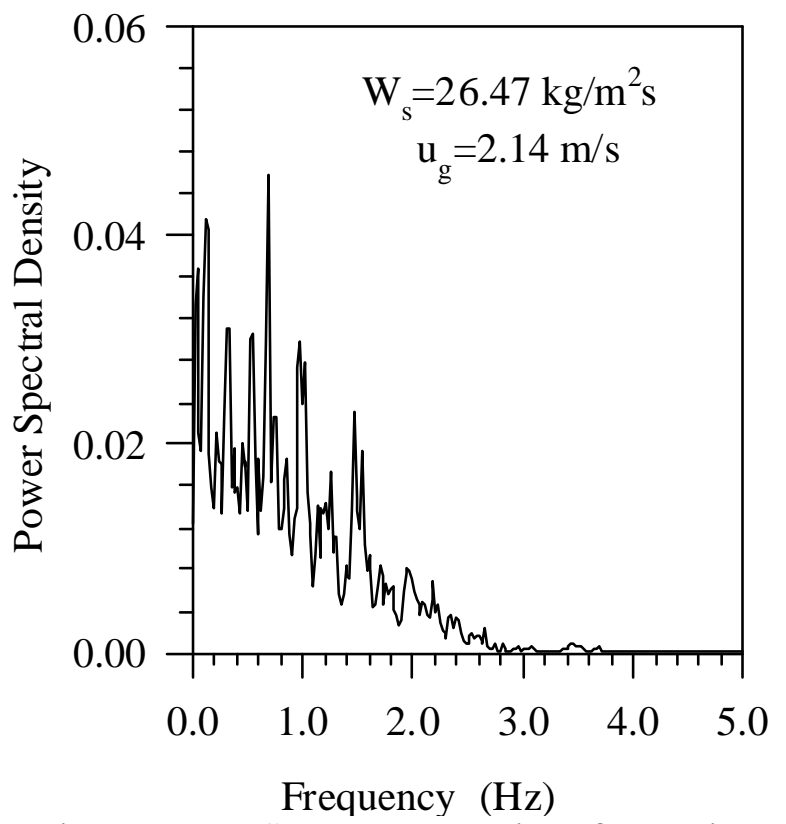

Fig. 7 Power Spectrum Density of Porosity

Fluctuations in the Dense Regime

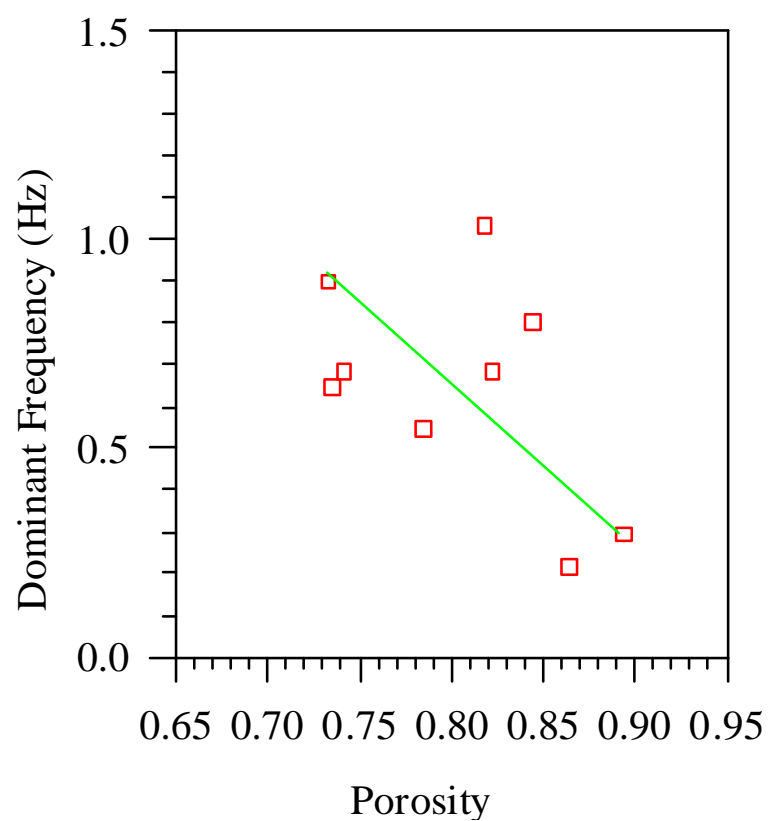

Fig. 8 Dominant Frequency in the Dense Phase Region

$\left(\mathrm{U}_{\mathrm{g}}=2.14 \mathrm{~m} / \mathrm{s}, \mathrm{W}_{\mathrm{s}}=26.47 \mathrm{~kg} / \mathrm{m}^{2} \mathrm{~s}\right.$; First Floor, Fig. $\left.1_{\mathrm{a}}\right)$

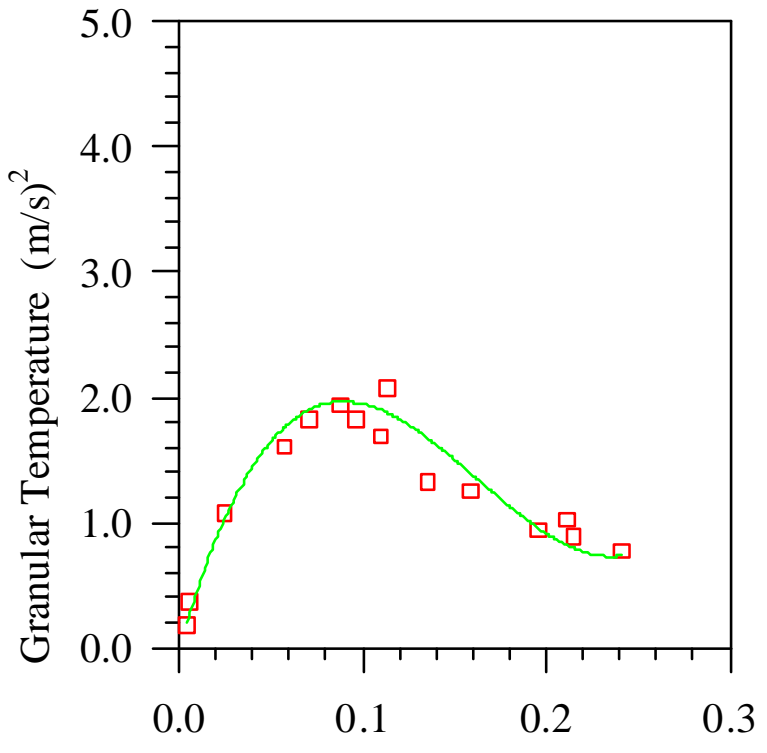

Solid Volume Fraction

Fig. 9 Granular Temperature for $75 \mu \mathrm{m}$ FCC Particles in the IIT CFB

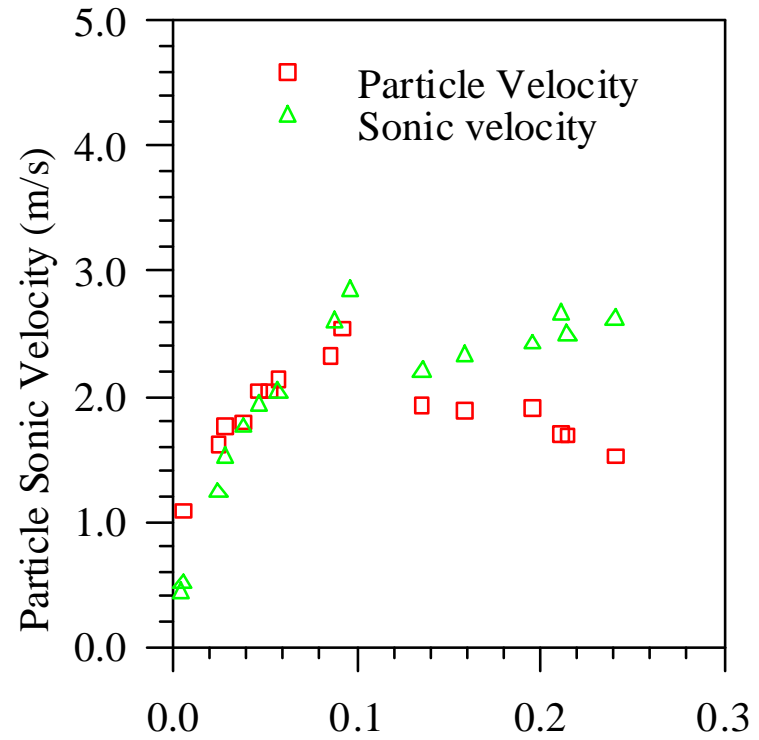

Solid Volume Fraction

Fig. 10 Particulate Sonic Velocities and Average Particle Velocities for $75 \mu \mathrm{m}$ FCC Particles in the IIT CFB 


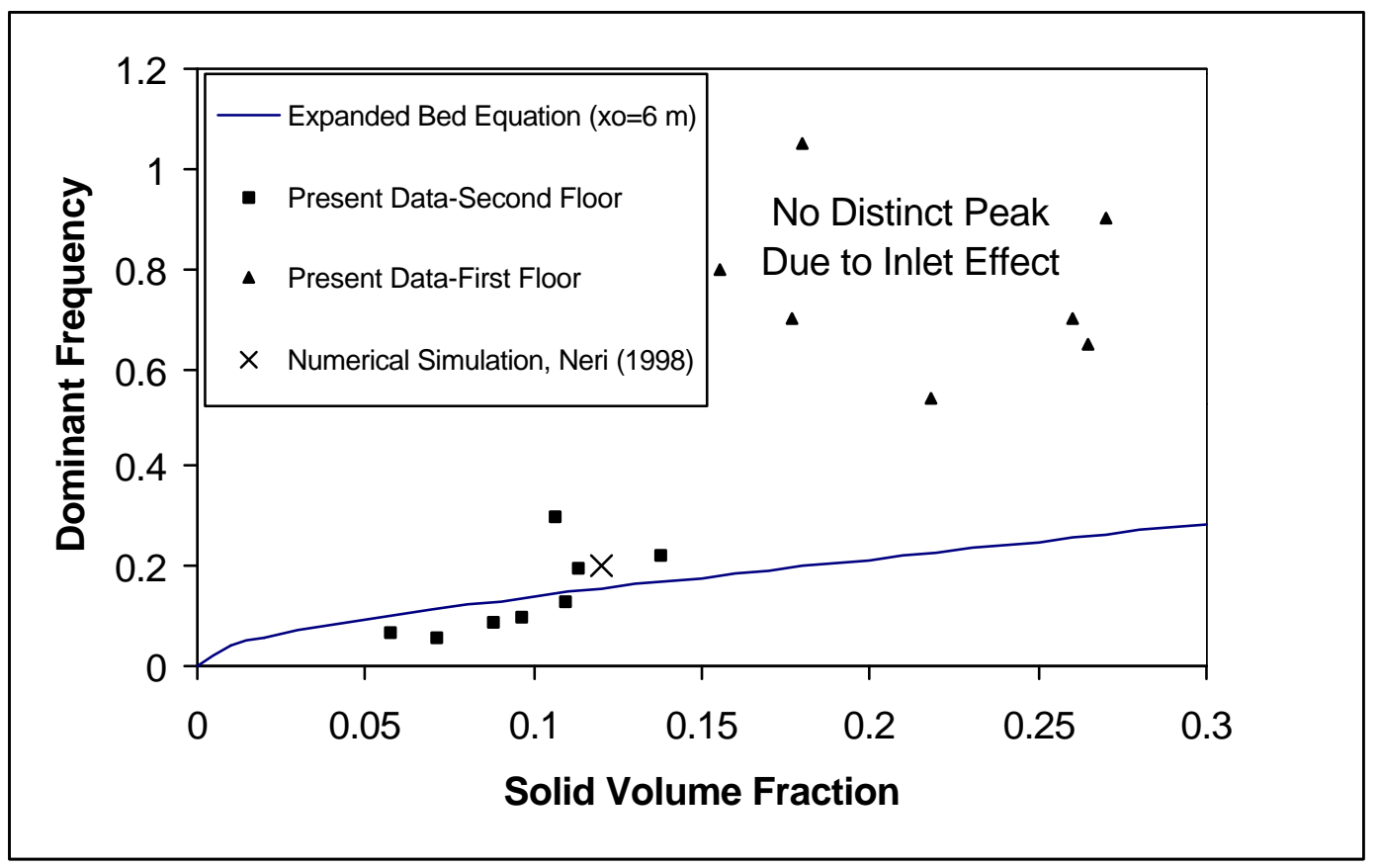

Figure 11- A Comparison of Riser Dominant Frequency, $f$ to the Analytical Solution

$$
f=\frac{1}{2 \pi}\left(\frac{g}{x_{o}}\right)^{\frac{1}{2}}\left[\frac{\left(3 \varepsilon_{s} / \varepsilon+2\right) \varepsilon_{s}}{\varepsilon_{s o}}\right]^{\frac{1}{2}} ; \quad \varepsilon_{\mathrm{so}}=0.5
$$



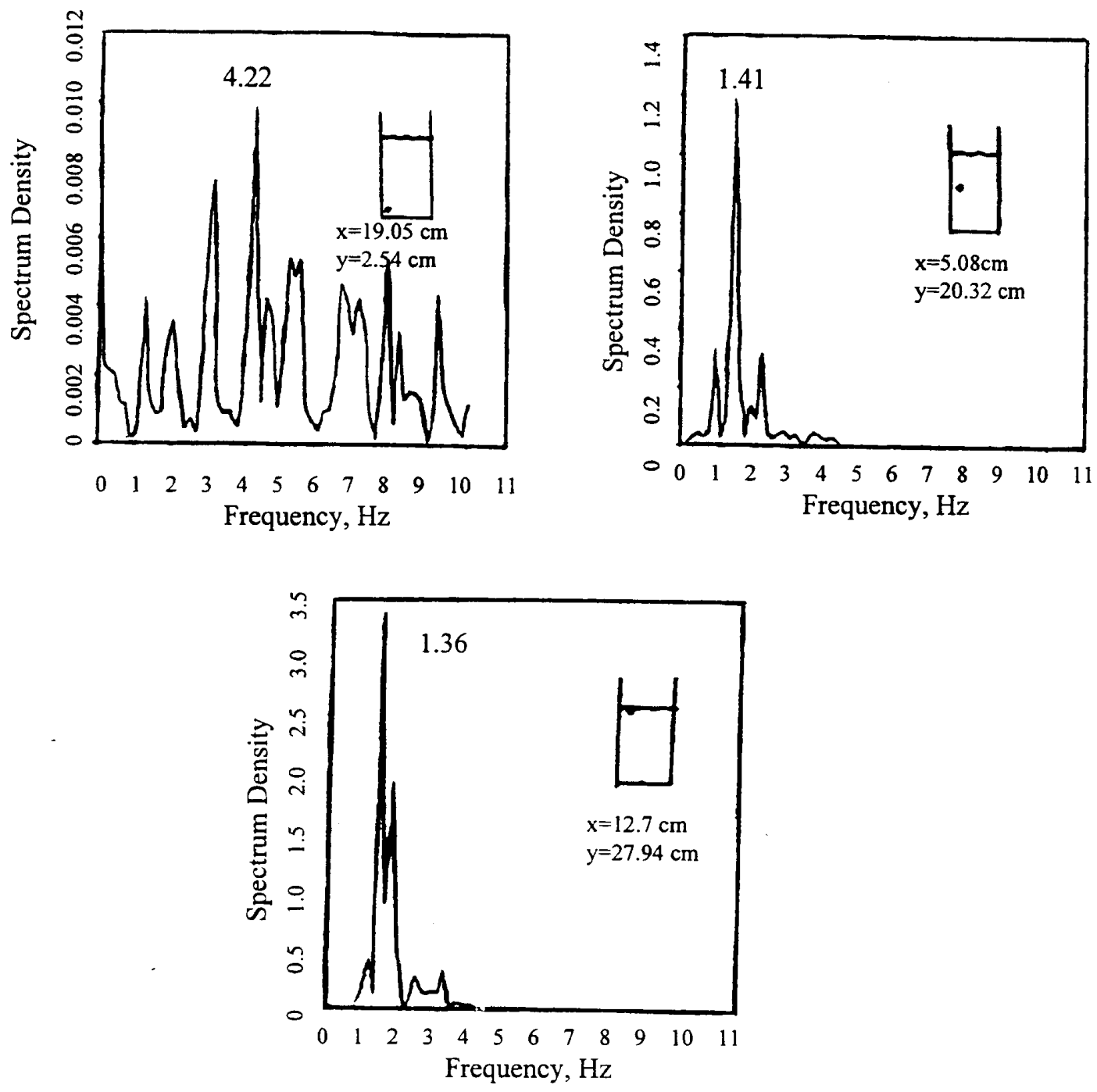

Figure 12 - Power Spectrum for Porosity Measured with a Gamma-ray Densitometer in the Two-Dimensional Bubbling Bed Described in Gidaspow's Book (Gidaspow, et al 1989 ) 


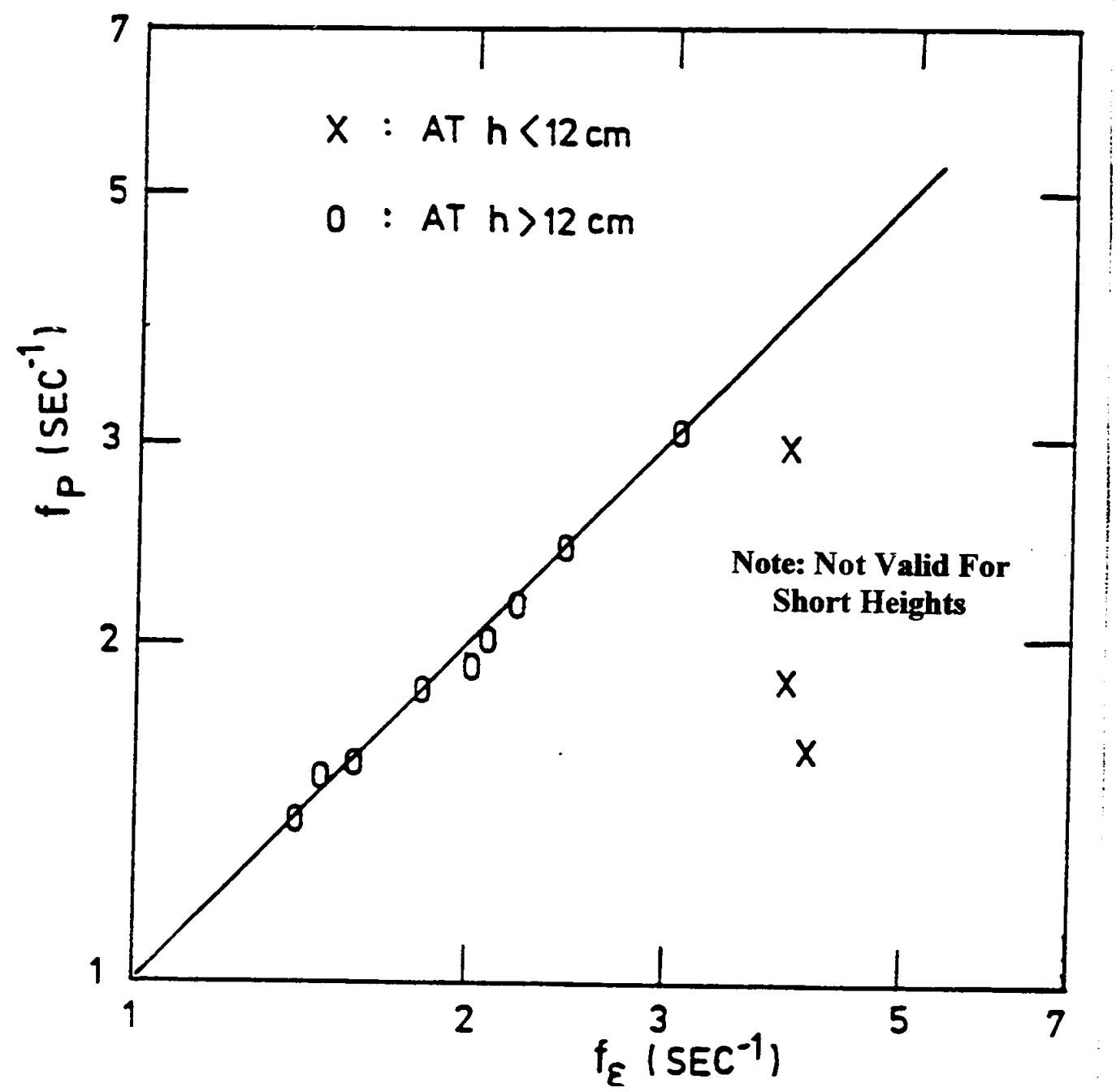

Figure 13. A Comparison of the Frequencies of Porosity Fluctuations to Pressure Fluctuations in a Two Dimensional Fluidized Bed with a Jet as Described in Gidaspow”s Book (Seo, 1985) 


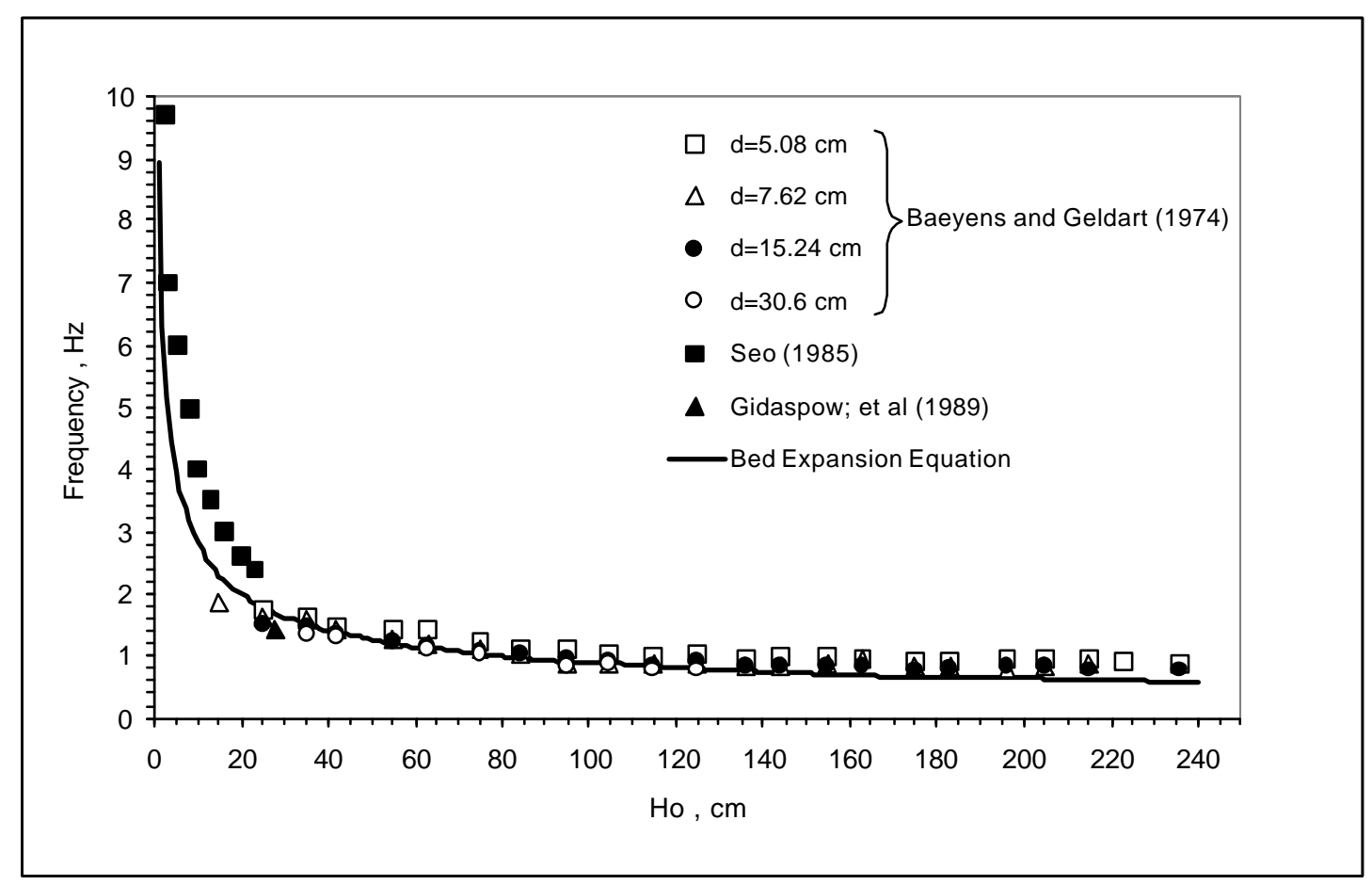

Figure 14. A Comparison of Dominant Bubbling Bed

Frequency Data to the Analytical Solution

$f=\frac{1}{2 \pi}\left(\frac{g}{x_{o}}\right)^{\frac{1}{2}}\left[\frac{\left(3 \varepsilon_{s} / \varepsilon+2\right) \varepsilon_{s}}{\varepsilon_{s o}}\right]^{\frac{1}{2}}$ 


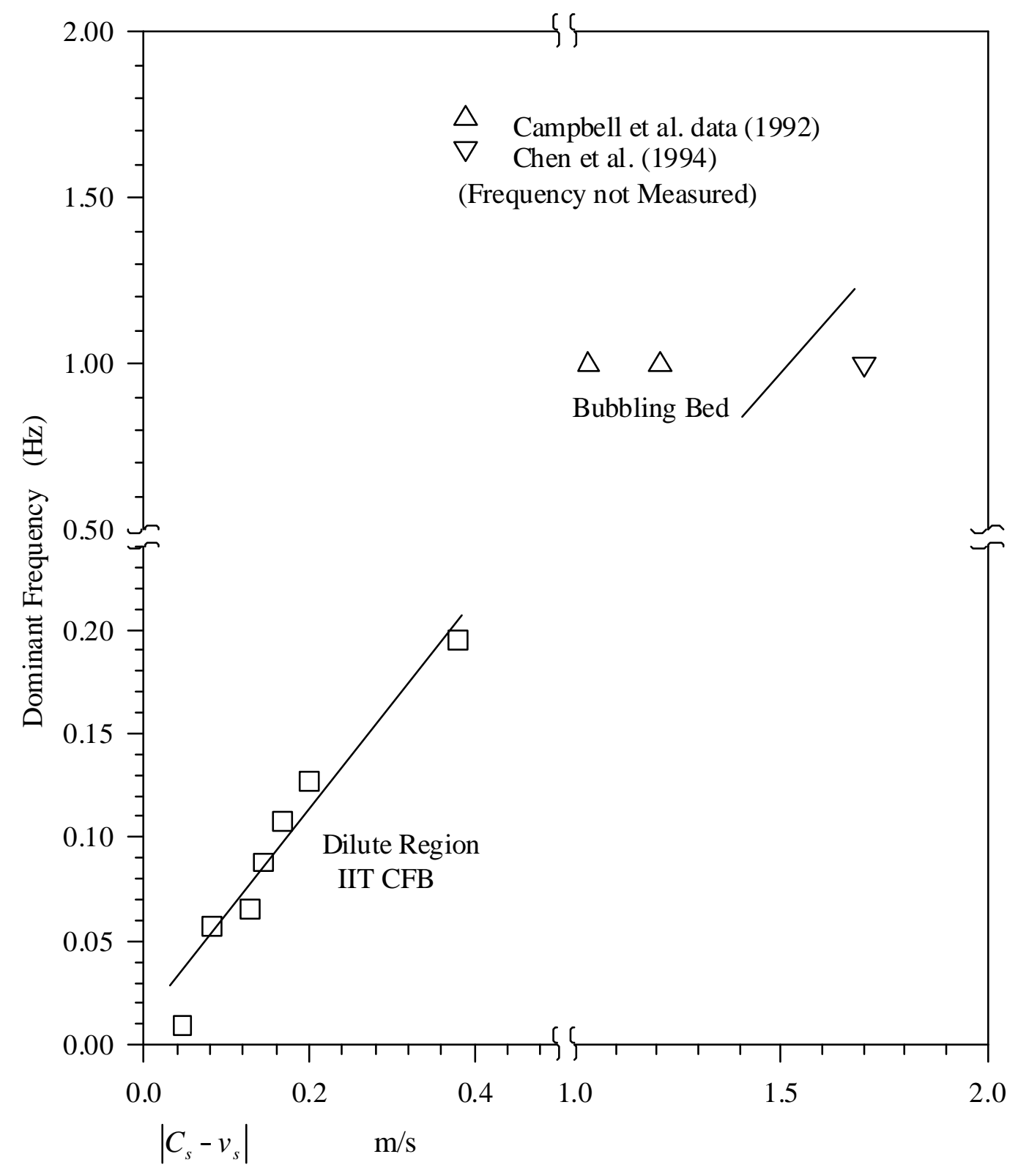

Figure 15 Kinetic Theory Explanation of Low Frequencies in The IIT CFB 


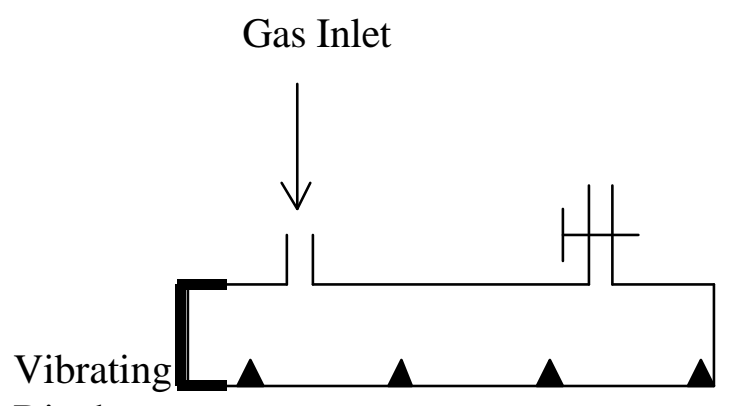

Diaphragm

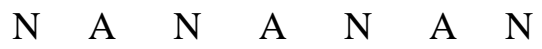

\begin{tabular}{|c|c|}
\hline $\begin{array}{l}\text { Standing Wave Solution } \\
\text { for a Narrow Tube }\end{array}$ & $\begin{array}{l}\text { wo Dimensions } \\
\text { oy, 1973) }\end{array}$ \\
\hline$V_{x}=\frac{3 V_{o}^{2}}{8 C_{s}} \sin 2 \alpha x$ & $\alpha=\frac{\omega}{C_{s}}$ \\
\hline
\end{tabular}

Figure 16. Kundt's Tube Solution

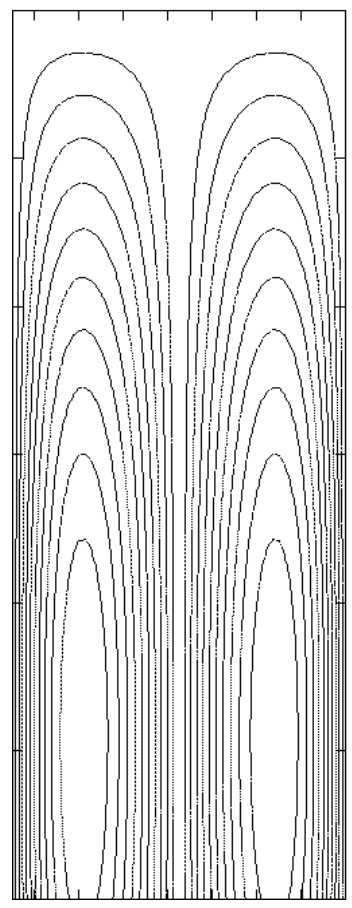

IIT Riser $(\mathrm{d}=7.5 \mathrm{~cm}, \mathrm{~h}=6 \mathrm{~m})$ $\mathrm{C}_{\mathrm{s}}=1.9 \mathrm{~m} / \mathrm{s}, \omega=0.3 \mathrm{~Hz}$

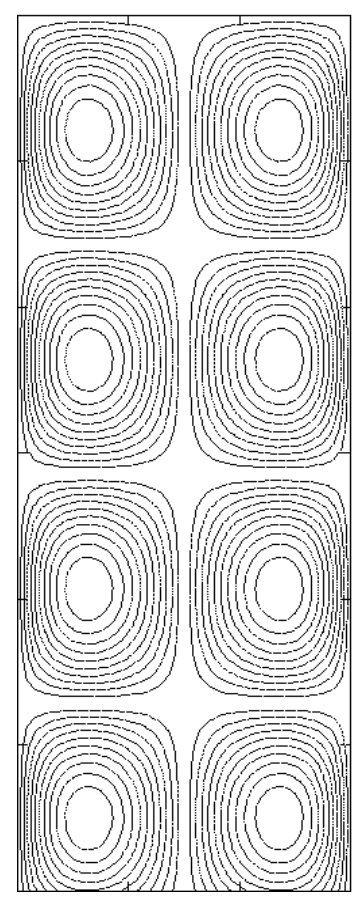

A Fluidized Bed $(\mathrm{l}=1.5 \mathrm{~m}, \mathrm{~h}=6 \mathrm{~m})$ $\mathrm{C}_{\mathrm{s}}=1 \mathrm{~m} / \mathrm{s}, \omega=1 \mathrm{~Hz}$

Figure 17. Flow Patterns Obtained from Standing Waves Analysis 


\title{
Turbulence of Particles in a CFB and Slurry Bubble Columns Using Kinetic Theory
}

\author{
Dimitri Gidaspow, Xiaolin Wu and M. Reza Mostofi \\ Department of Chemical and Environmental Engineering \\ Illinois Institute of Technology, Chicago, IL 60616 \\ Fax: 312-567-8874, E-mail: gidaspow@iit.edu
}

Presented at the AIChE 1999 Annual Meeting, Dallas Texas, November 3, 1999

The objective of this research is to understand turbulence in circulating fluidized beds (CFB) and bubble column reactors for the purpose of improving multiphase models, such as described in Gidaspow's book, which can be used for reactor design. Recently Buyevich and Cody (1998) found that the granular temperature of particles in bubbling fluidized beds is a maximum at a diameter of about 90 microns and have given an explanation for this observation. The heat transfer coefficient has a similar behaviour. To find an optimum particle size and to confirm and extend Cody's observations, the granular temperature was measured in the IIT CFB for 450 micron glass beads. The granular temperature was measured in the two-story IIT CFB ( Gidaspow and Huilin, 1996) using the CCD camera method described earlier. Figures 1 and 2 show the typical axial and tangential velocity distributions measured with the camera. The local volume fraction was also measured with the camera using the method of Gidaspow and Huilin (1998a) that was shown to give densities close to those determined with an X-ray densitometer. From the standard deviations, such as those seen in Figures 1 and 2, the granular temperature was obtained, assuming equality of tangential and radial standard deviations. Figure 3 shows the granular temperature behavior for the 450 micron glass beads. The shape of the curve is similar to that for 75 micron FCC particles (Gidaspow and Huilin, 1996), but the granular temperature is one order of magnitude lower. This surprising result can be explained on the basis of a balance of production of granular temperature due to shear and dissipation due to inelasticity, which shows that (Gidaspow, 1994, Eq 10. 12)

Granular temperature $=$ constant $\times(\text { shear rate } \times \text { particle diameter })^{2}$

The shear rate for 450 micron beads was measured to be $13 \mathrm{sec}^{-1}$ at a volume fraction of 0.04 by means of a probe. This shear rate is only roughly one twentieth that of FCC particles. This shear rate is much lower for 450 micron beads due to the higher slip. Hence the shear rate decreased faster than the diameter increased, leading to the surprisingly lower turbulence.

The velocity measurements also allowed us to compute the Reynolds stress from the product of the deviations of the velocities in the two directions. Fig. 4 depicts the results. Since the tangential velocity has a larger error than the velocity in the direction of flow, there is more error in the Reynolds stress than in the granular temperature. The ratio of the Reynolds stress to the granular temperature gives us an estimate of the restitution coefficient. We need this value as an input into our kinetic theory based hydrodynamic model. Most of the values are in the range of 0.99 to 0.999 . 
Figure 5 shows the viscosity of the 450 micron beads. It was computed in two ways: 1. Using the kinetic theory formula, Eq. 5 in Gidaspow and Huilin(1996) with the radial distribution function at contact given by the Bagnold formula, Eq 7. 2. From the equation,

Reynolds stress $\times$ particle density $\times$ solids volume fraction $=$ viscosity $\times$ shear gradient.

Similarly to the results reported at the 1998 Miami Beach annual AlChE meeting (Gidaspow, et al 1998) the viscosity calculated from the Reynolds stress and from the granular temperature agree with each other. As expected, the viscosity of the large beads is higher than the FCC viscosity. However, the viscosity of the 450 micron beads in air in our CFB is an order of magnitude higher than the viscosity of the beads in water reported in the Miami Beach PTF Proceedings (Gidaspow, et al 1998) due to the lower granular temperature in water. See Fig. 6. Fig. 7 compares the velocity gradients in the CFB in air and in water in the fluidized bed with a central jet. The higher granular temperature and the viscosity in air cannot be explained based on the lower shear rate. The difference must be due to the lower air viscosity compared with water. Particles oscillate much faster in air than in water for the same shear rate.

These results are useful for understanding turbulence in slurry bubble column reactors. Fig. 8 shows a comparison of our computed granular temperature using the model and code reported by $\mathrm{Wu}$ and Gidaspow (1999) to experimental data for 45 micron Air Products methanol catalyst particles. We had used the restitution coefficient obtained from our experiment in the simulation. Fig 9 shows the computed solids viscosity. It is quite small due to the low granular temperature and the small catalyst size. In Fig. 8, it is the flow of gases that produced the turbulence. The granular temperature of the catalyst particles in water was almost an order of magnitude lower ( Gidaspow and Huilin, 1998b).

\section{Acknowledgment}

This study was supported by the National Science Foundation Fluid, Particulate and Hydraulic System Grant No. CTS-9610053 and by the Department of Energy Grant No. DE-FG-98FT40117.

\section{References}

Buyevich Y. A. and Cody G.D. 1998, Particle fluctuations in homogeneous fluidized beds, Prep. for Brighton World Congress on Particle Tech., 3, Brighton, UK, Paper 207.

Gidaspow D. and Huilin L., 1996, Collisional viscosity of FCC particles in a CFB, AIChE. J. 42, 2503-2510.

Gidaspow D. and Huilin L., 1998a, Equation of state and radial distribution function of FCC particles in a CFB, AIChE. J. 44, 279-293.

Gidaspow D. and Huilin L., 1998b, A comparison of gas-solid and liquid-solid fluidization using kinetic theory and statistical mechanics, Fluidization IX, edited by L. S. Fan and T. M. Knowlton, New York: Engineering Foundation, 662-668.

Gidaspow D. 1994, Multiphase flow and fluidization: Continuum and kinetic theory descriptions, Boston: Academic Press Inc.

Gidaspow D., Guo Y. and Mostofi M. R. 1998, Turbulence in liquid-solid fluidization, AIChE annual meeting preprints, Vol. II, Particte Technology Forum, Miami Beach, $15-20$. 
Wu Y. and Gidaspow D. 1999, Hydrodynamic simulation of methanol synthesis in gasliquid slurry bubble column reactors, to appear in Chem. Eng. Science. 


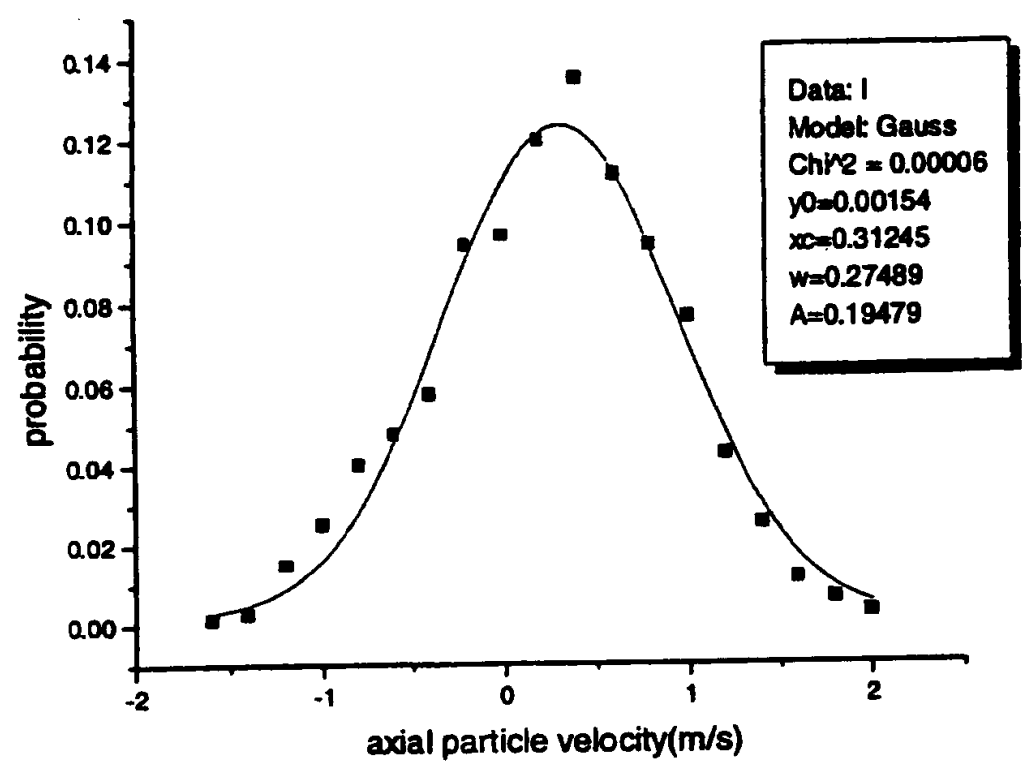

Figure 1. Axial Particle Velocity Distribution For $450 \mu \mathrm{m}$ Glass Beads

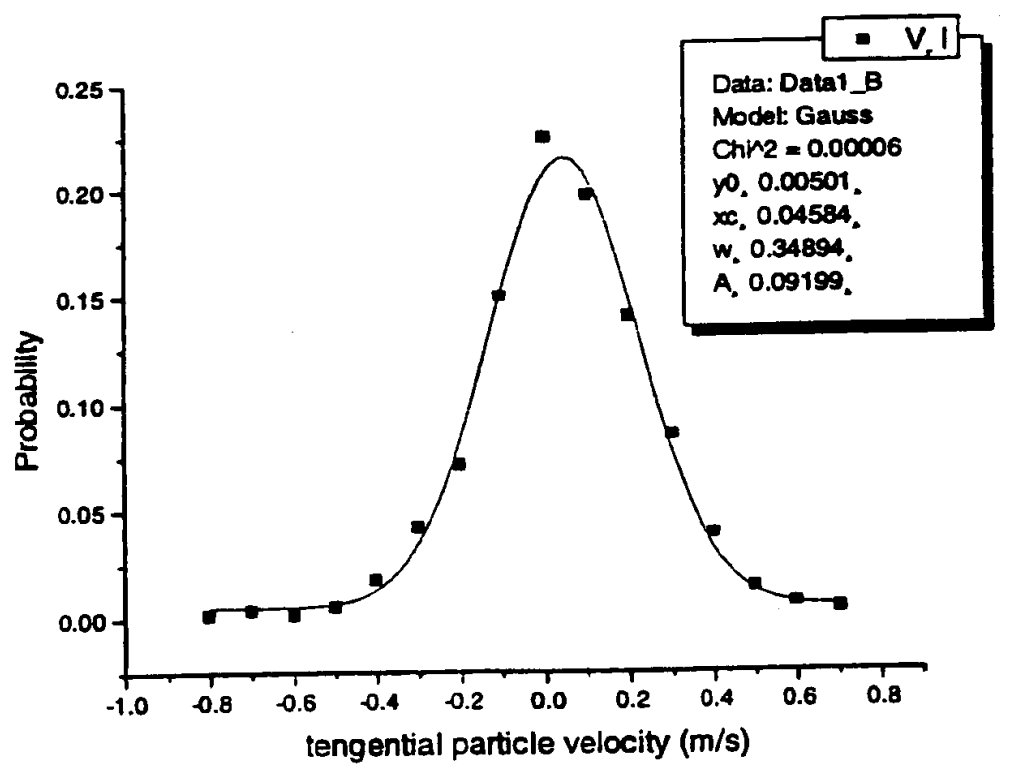

Figure 2. Tangential Particle Velocity Distribution For $450 \mu \mathrm{m}$ Glass Beads 


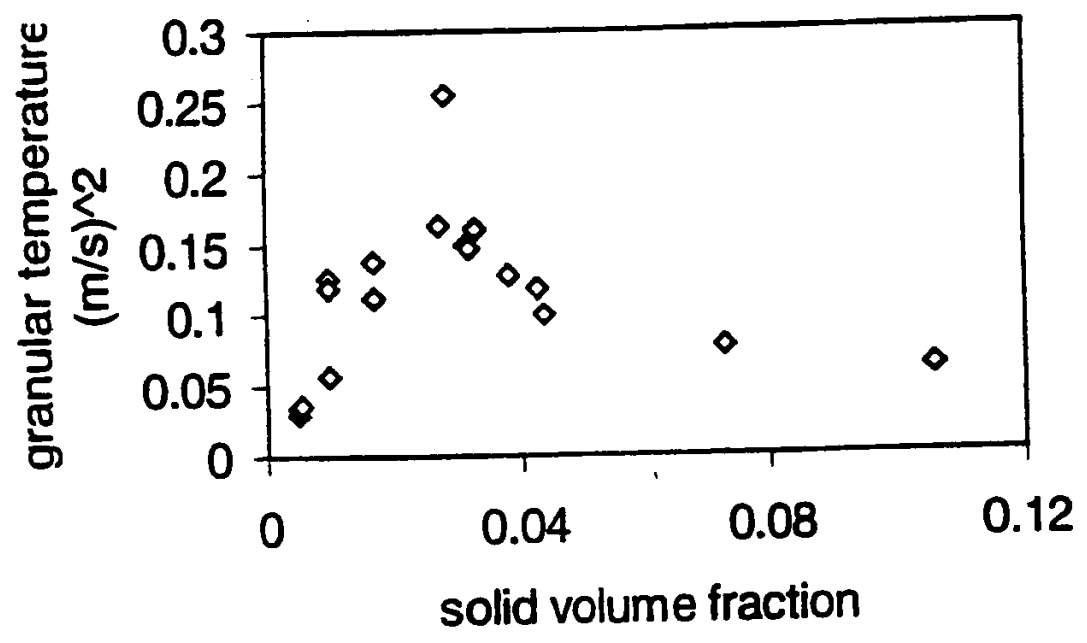

Figure 3. Granular Temperature, $\theta=\frac{1}{3}\left(2 \sigma_{\theta}^{2}+\sigma_{3}^{2}\right)$

For $450 \mu \mathrm{m}$ Glass Beads

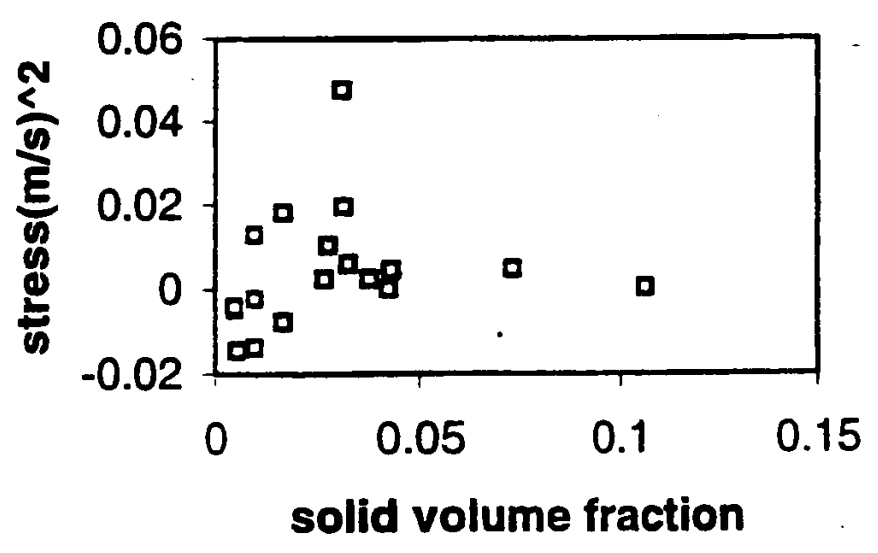

Figure 4. Reynolds Stress, $\overline{v_{\theta}^{\prime} v_{:}^{\prime}}=\frac{1}{N} \sum_{n=1}^{N}\left(v_{\theta}-\bar{v}_{\theta}\right)\left(v_{z}-\bar{v}_{z}\right)$

For $450 \mu \mathrm{m}$ Glass Beads 


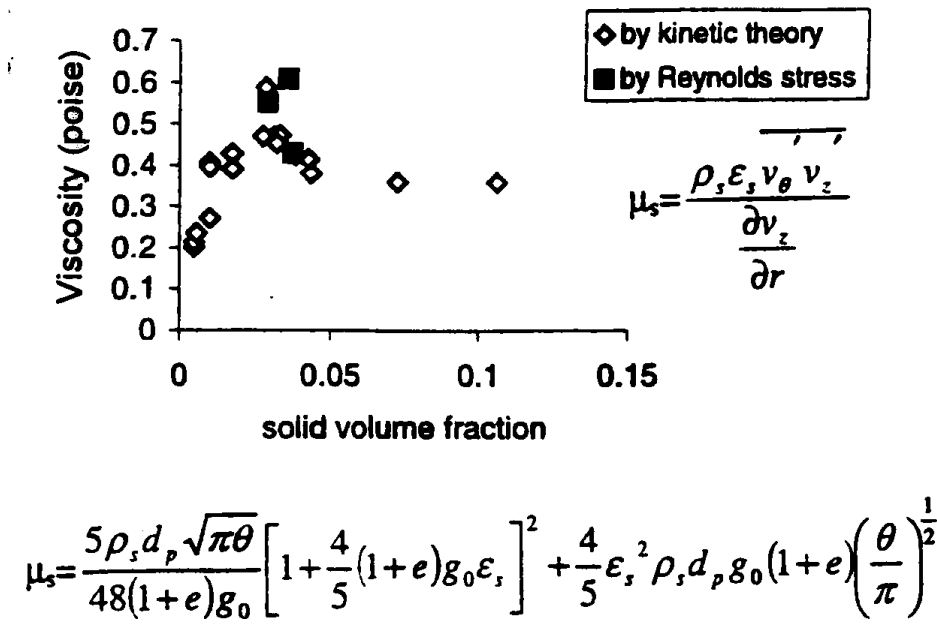

Figure 5. Solids Viscosity For $450 \mu \mathrm{m}$ Glass Beads

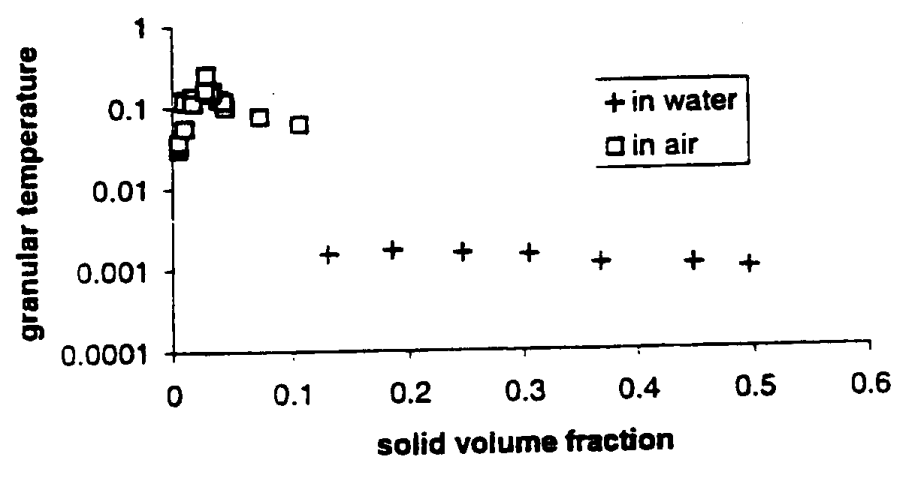

Figure 6. A Comparison of Granular Temperature of $450 \mu \mathrm{m}$ Glass Beads in Air in the IIT CFB and in a Water Fluidized Bed (Gidaspow, et al 1998)

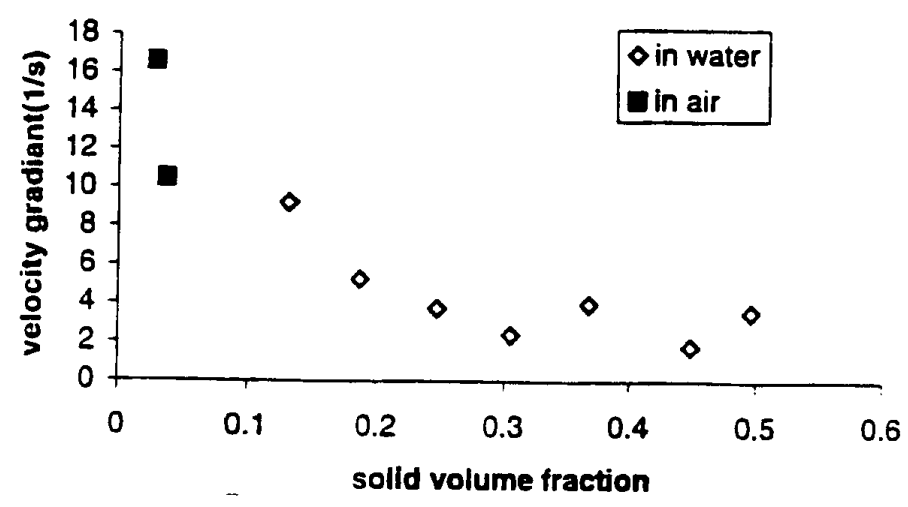

Figure 7. Velocity Gradient of $450 \mu \mathrm{m}$ Glass Beads in Different Media 


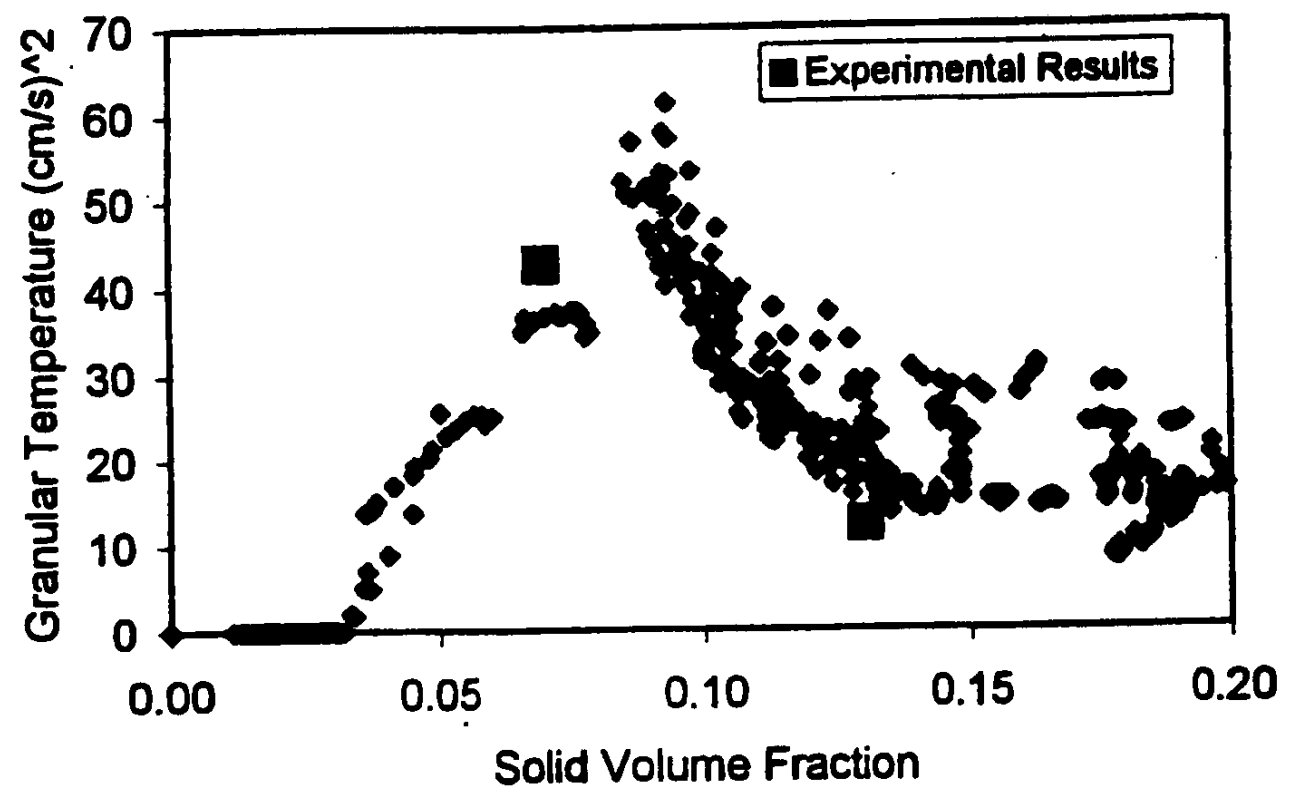

Figure 8. A Comparison of Computed Granular Temperature in a Slurry Bubble Column Reactor (Wu and Gidaspow, 1998) to Experiments with $45 \mu \mathrm{m}$ Catalyst

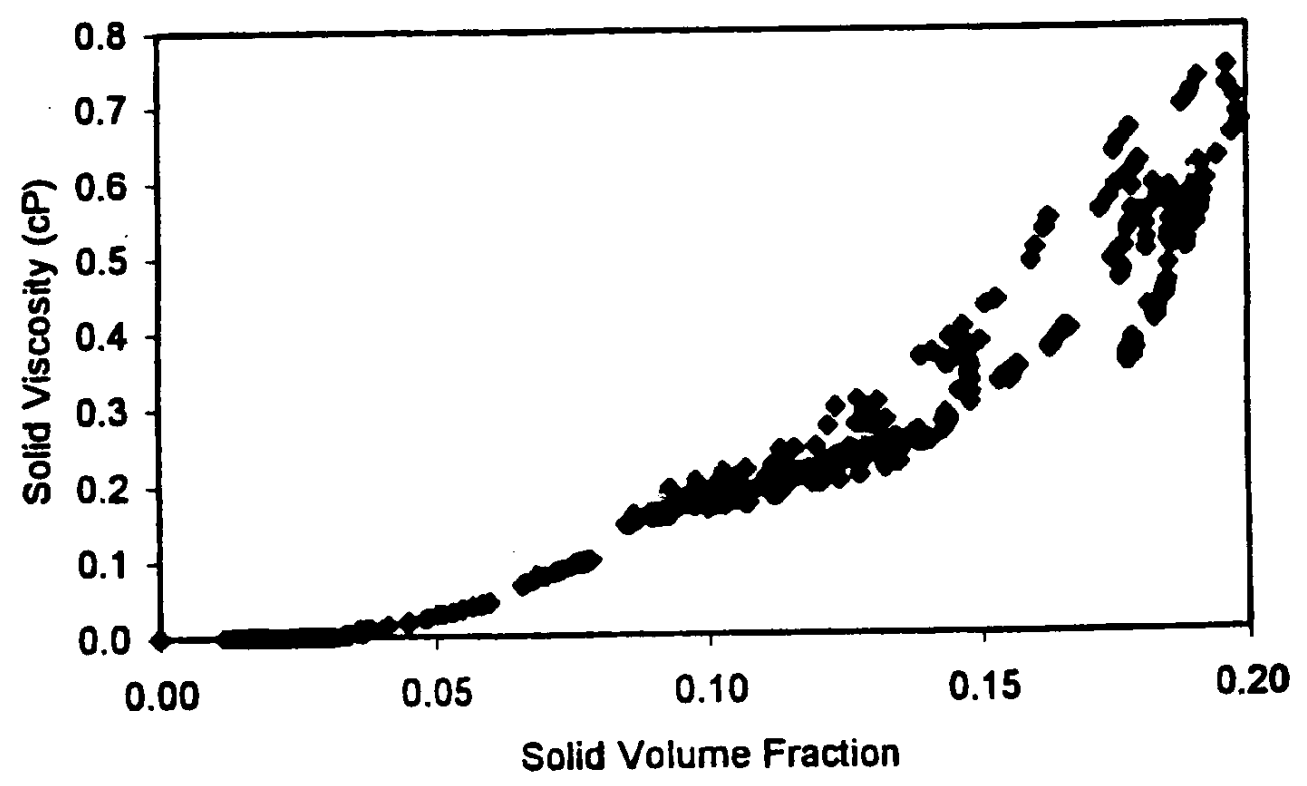

Figure 9. Computed Viscosity of $45 \mu \mathrm{m}$ Catalyst 


\section{ARTICLES AND PRESENTATIONS}

Journal Articles

- Matonis D., D. Gidaspow and M. Bahary, "CFD Simulation of Flow and Turbulence in a Slurry Bubble Column Reactor", in press AIChE Journal, also presented in ICMF 2001, New Orleans, Louisiana, May 2001.

- $\quad \mathrm{Wu}$ Y. and D. Gidaspow, "Hydrodynamic simulation of methanol synthesis in gasliquid slurry bubble column reactors", Chem. Eng. Sci., 55, 573-587, 2000.

Conference Presentations

- Gidaspow D., "Fluidized Bed Hydrodynamics and Direct Contact Heat Transfer using Kinetic Theory", Festschrift session, $33^{\text {rd }}$ National Heat Transfer Conference, Albuquerque, New Mexico, Aug. 15-17, 1999.

- Gidaspow D., Wu Y. and R. Mostofi, "Turbulence of Particles in a CFB and Slurry Bubble Columns using Kinetic Theory", Fluidization and Fluid-Particle Systems Preprint Volume, AIChE, pp. 261-266, AIChE Annual meeting, Dallas, Texas, Oct. 31-Nov. 5, 1999.

- Gidaspow D., Mostofi R., Tartan M., Vijayaraghavan K., Sharma B., Matonis D. and Y.S. Shin, "Computation and Measurement of Structure and Turbulence in Risers and Bubbling Beds", Multiphase Fluid Dynamics Research Consortium Annual Meeting, Albuquerque, New Mexico, Apr. 12, 2000.

- Gamwo I., Halow J.S., Gidaspow D., Mostofi R. and Matonis D., "CFD models for slurry bubble column reactors", $5^{\text {th }}$ International Conference on Gas-Liquid and GasLiquid-Solid Reaction Engineering World Congress, Melbourne, Australia

- Tagny, J., and Lam, P., "Influence of Thermal Radiation on Natural Convection Heat Transfer in a Pipe", Book of Abstracts, pp. 126, $29^{\text {th }}$ Annual Conference, NOBCChE March 25-30, 2002, New Orleans, Louisiana.

- Gidaspow, D., " Fluidized Bed Hydrodynamics and Direct Contact Heat Transfer Using Kinetic Theory', 33 ${ }^{\text {rd }}$ National Heat Transfer Conference, August 15-17, 1999 , Albaquerque, New Mexico. 


\section{Appendix}

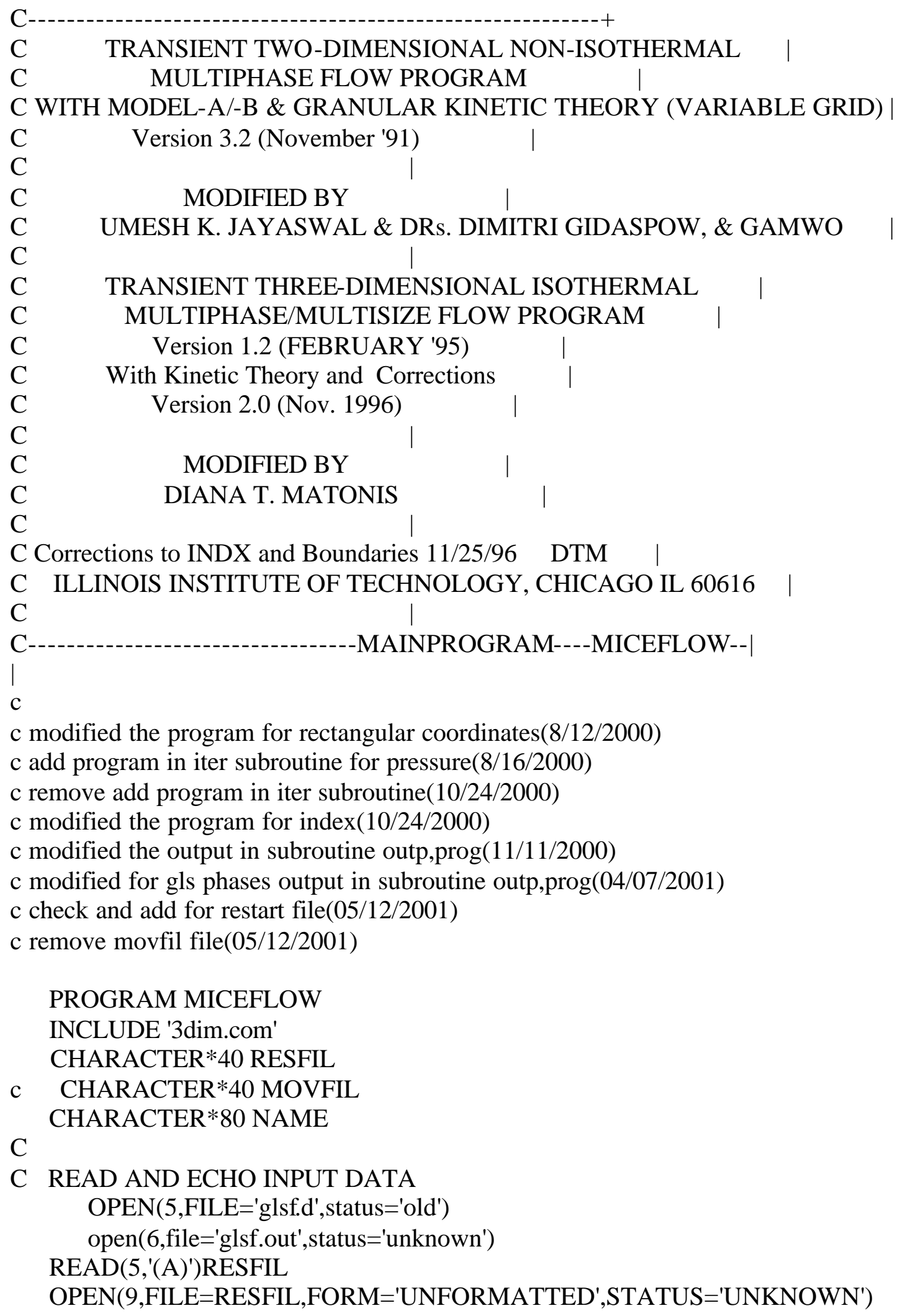


c $\left.\quad \operatorname{READ}\left(5,,^{\prime}(\mathrm{A})\right)^{\prime}\right) \mathrm{MOVFIL}$

c OPEN(10,FILE=MOVFIL,STATUS='UNKNOWN')

$\operatorname{READ}\left(5,{ }^{\prime}(\mathrm{A})^{\prime}\right) \mathrm{NAME}$

WRITE(6,'(A)')' MULTIFLOW PROBLEM IDENTIFIER - ',NAME

$\operatorname{READ}\left(5,{ }^{*}\right) \mathrm{ITC}, \mathrm{IB} 2, \mathrm{JB} 2, \mathrm{~KB} 2, \mathrm{NSOLID}$

WRITE(6,210)ITC,IB2,JB2,KB2

C

$$
\begin{aligned}
& \text { NPHASE=NSOLID+1 } \\
& \text { IB=IB2-2 } \\
& \text { IB1=IB2-1 } \\
& \text { JB=JB2-2 } \\
& \text { JB1=JB2-1 } \\
& \text { KB = KB2-2 } \\
& \text { KB1 = KB2-1 } \\
& \text { IB2JB2=IB2 } * J B 2 * K B 2 \\
& \text { IB2JB1=IB2JB2-IB2 } \\
& \text { IB1JB2=IB2JB2-1 }
\end{aligned}
$$

C

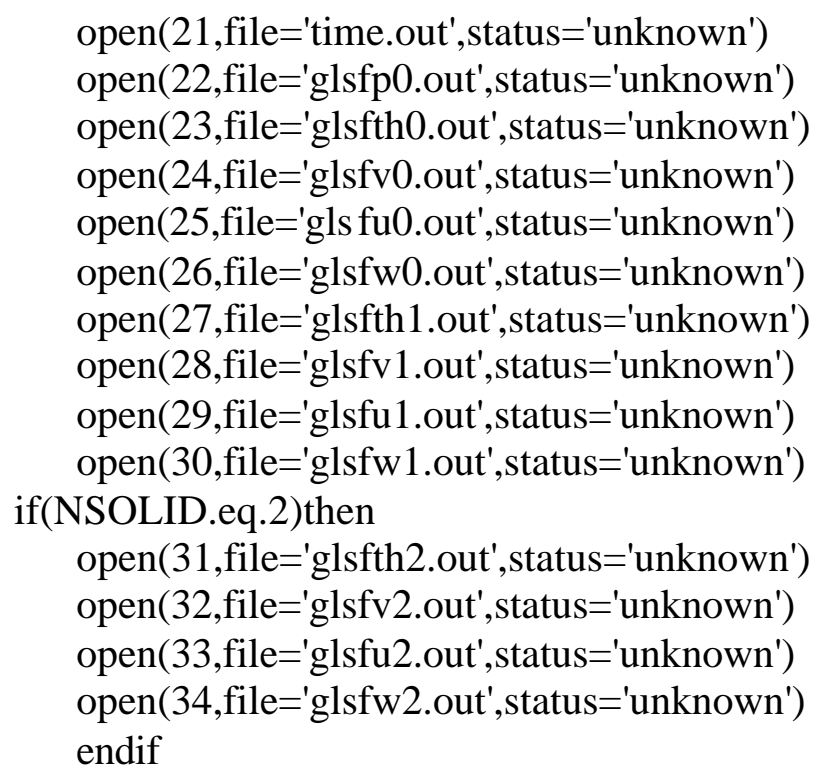

$\operatorname{READ}\left(5,{ }^{*}\right) \mathrm{RST},(\mathrm{DR}(\mathrm{I}), \mathrm{I}=1, \mathrm{IB} 2)$

WRITE(6,215)RST,(DR(I),I=1,IB2)

$\operatorname{READ}(5, *)(\mathrm{DZ}(\mathrm{J}), \mathrm{J}=1, \mathrm{JB} 2)$

WRITE $(6,216)(\mathrm{DZ}(\mathrm{J}), \mathrm{J}=1, \mathrm{JB} 2)$

$\operatorname{READ}(5, *)(\mathrm{DY}(\mathrm{L}), \mathrm{L}=1, \mathrm{~KB} 2)$

WRITE(6,217)(DY(L),L=1,KB2)

READ $(5, *) N C O N T, M O D A B, I P R E S, I N E N T, I K I N T, I C O H, I S W I T, I F L Z B$

WRITE(6,320)NCONT,MODAB,IPRES,INENT,IKINT,ICOH,ISWIT

1, IFLZB

WRITE(6,250)NPHASE

DO $6 \mathrm{~K}=0$,NSOLID 


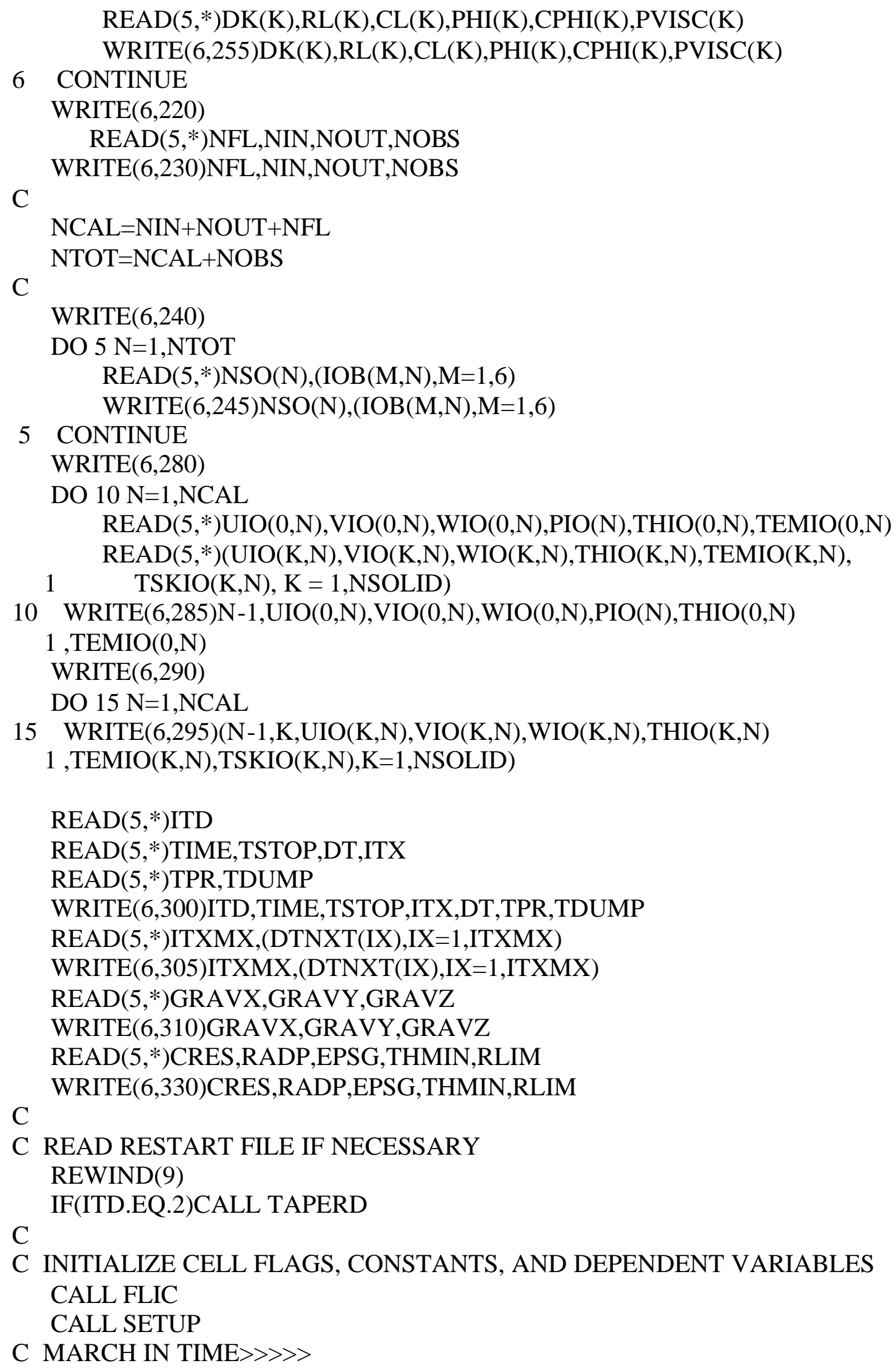




\section{CALL PROG}

STOP

210 FORMAT(' 1. GEOMETRY'/' A. COORDINATES (0- CARTESIAN,'

1 , ' 1- CYLINDRICAL, 2- SPHERICAL)=',I2/' B. MESH SIZE',

1 ' R (or X)-DIR, IB2=',I3,', ','Z (or Y)-DIR, JB2='

1 ,I3/,17X,' THETA(or Z)-DIR, KB2=',I3/' C. CELL SIZES')

215 FORMAT(7X,'DISTANCE OF FIRST CELL FROM CENTER = ',F10.5,

1 ' $\mathrm{cm} ' / 7 \mathrm{X}$,'IN R (or X)-DIRECTION, DR (cm) ='/6(2X,F9.5))

216 FORMAT(7X,'IN Z (or Y)-DIRECTION, DZ (cm) ='/6(2X,F9.5))

217 FORMAT(7X,'IN THETA (or Z)-DIRECTOION, DY (cm) ='/6(2X,F9.5))

320 FORMAT(/' 2. MODELS: (=0- NO, >0- YES)'/10X,'\# OF ',

1 'CONTINUOUS PHASES (1- GAS/LIQUID, 2- GAS\&LIQUID) = ',I2

$1 / 10 X$, 'MODEL (1- A, 2- B)= ',I2/10X,'INITIAL ',

1 'PRESSURE PROFILE (0- FLUID WT., 1- BED WT.) = ',I2/10X

1 ,'ENERGY EQUATIONS (1- INTERNAL ENERGY, 2- ENTHALPY)='

1, I2/10X,'KINETIC THEORY OF GRANULAR SOLIDS MODEL=',I2

$1 / 10 \mathrm{X}$, 'COHESIVE FORCE MODEL=',I2/10X,'SWITCH CONTINUOUS'

1, , \& DISPERSED PHASES (0- NO, 1 - YES) = ',I2,

$1 / 10 \mathrm{X}$, 'FLUIDIZED BED $(0-\mathrm{NO}, 1$ - YES) = ',I2)

250 FORMAT(/' 3. DATA FOR ',I2,' PHASES :'/4X,'DIAMETER ',

1 'DENSITY HEAT SPHERICITY PACKING ',

1 'VISCOSITY'/26X,'CAPACITY',16X,'FRACTION COEFFICIENT'/

$\left.16 \mathrm{X},,^{\prime}(\mathrm{cm})^{\prime}, 5 \mathrm{X},{ }^{\prime}\left(\mathrm{g} / \mathrm{cm}^{\wedge} 3\right) \quad(\mathrm{erg} /(\mathrm{g} . \mathrm{K}))^{\prime}, 25 \mathrm{X},{ }^{\prime}(\mathrm{g} /(\mathrm{cm} . \mathrm{s}))^{\prime}\right)$

255 FORMAT(2X,2(2X,G9.3),1X,G10.3,4X,F6.4,5X,G9.4,1X,G10.3)

220 FORMAT(/' 4. CELL FLAGS'/6X,'(1- FLUID, 2- FREE SLIP,',

1 ' 3- NO SLIP, 4- OUTFLOW (dP|exit = 0)'/7X,'5-',

1 ' INFLOW (FLOW \& P), 6- INFLOW, 7- OUTFLOW (P|exit',

1 ' = const)'/7X,'8- FLUID OUTFLOW $(\mathrm{P} \mid$ exit = const) $\left.))^{\prime} /\right)$

C $1^{\prime}$ A. BOUNDARIES'/7X,'BOTTOM=',I3,

C 1' LEFT=',I3,' TOP=',I3,' RIGHT=',I3)

230 FORMAT(4X,'A. NUMBERS OF: FLUID BLOCKS =',I2,

1 ', INFLOW BLOCKS=',I2/20X,'OUTFLOW BLOCKS=',I2,

1 ', OBSTACLE BLOCKS=',I2)

240 FORMAT(7X,'FLAG',5X,'--------COORDINATES---------')

245 FORMAT(5X,I3,6(4X,I5))

280 FORMAT( $/$, ' B. INFLOW - OUTFLOW DATA (FLUID)'/' BLOCK',

1 ' UIO',8X,'VIO',8X,'WIO',8X,'PIO',7X,'THIO',7X,'TEMIO'

$1 / 7 \mathrm{X},(\mathrm{cm} / \mathrm{s})^{\prime}, 5 \mathrm{X},{ }^{\prime}(\mathrm{cm} / \mathrm{s}) \quad(\mathrm{cm} / \mathrm{s}) \quad(\text { dynes/cm²})^{\prime}, 11 \mathrm{X}$,

1 '(Kelvin)')

285 FORMAT(1X,I2,1X,6(1PE11.3),2(1PE10.3))

290 FORMAT( $/, '$ C. INFLOW - OUTFLOW DATA (SOLIDS)' $/$

1 ' BLOCK/',3X,'UPIO',7X,'VPIO',7X,'WPIO',7X,'THPIO',5X,

1 'TEMPIO',6X,'TSKIO'// PHASE',3X,'(cm/s)',5X,'(cm/s)',5X,'(cm/s)'

$1,15 \mathrm{X},($ Kelvin)',2X,'((cm/s)^2)')

295 FORMAT((1X,I2,'/',I1,1X,6(1X,1PE10.3)))

300 FORMAT(/,' 5. CONTROL'/ 3X,'A. DUMP AND RESTART:', 


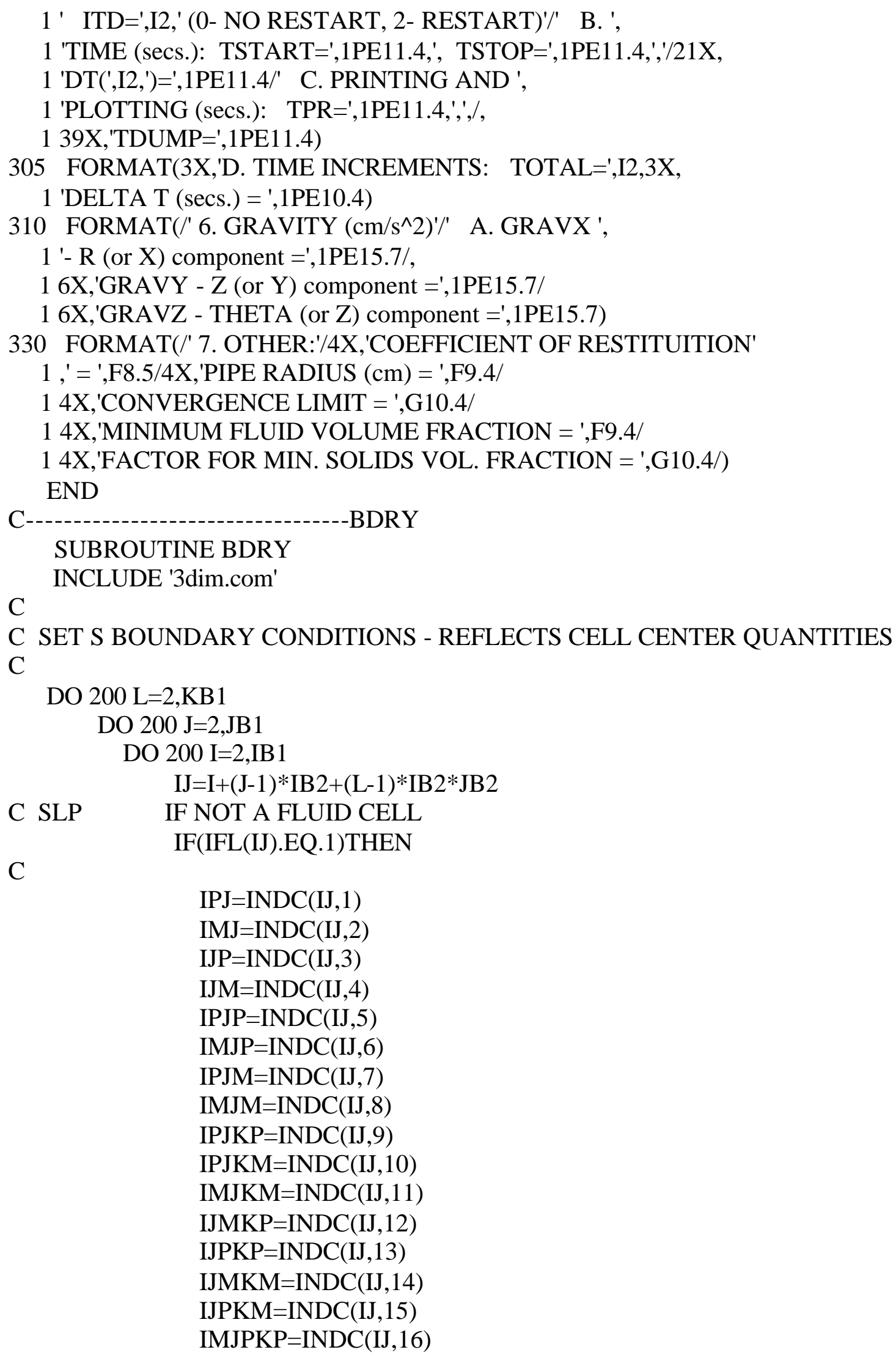




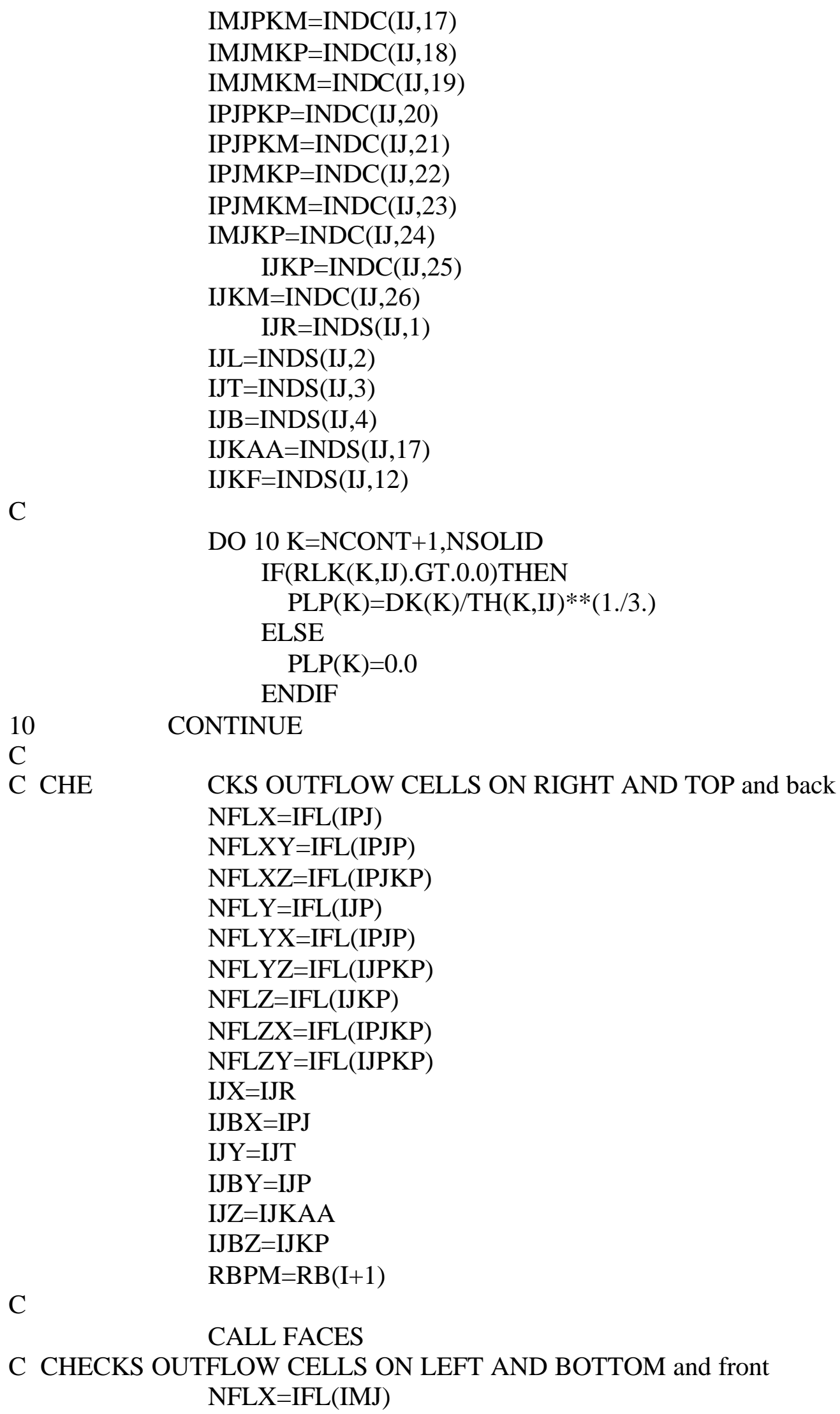

CALL FACES

C CHECKS OUTFLOW CELLS ON LEFT AND BOTTOM and front NFLX $=$ IFL(IMJ) 


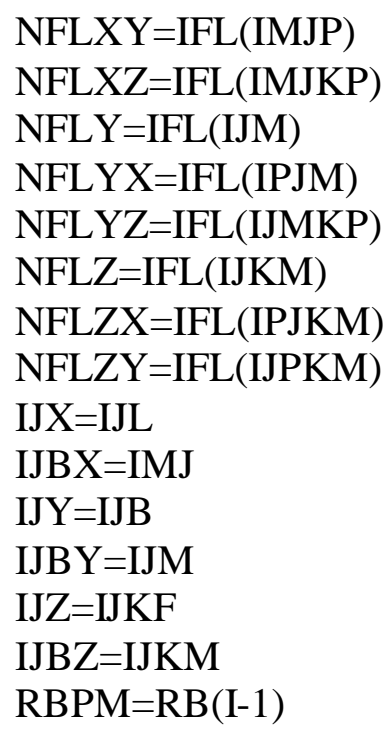

C

CALL FACES

ENDIF

\section{CONTINUE}

RETURN

END

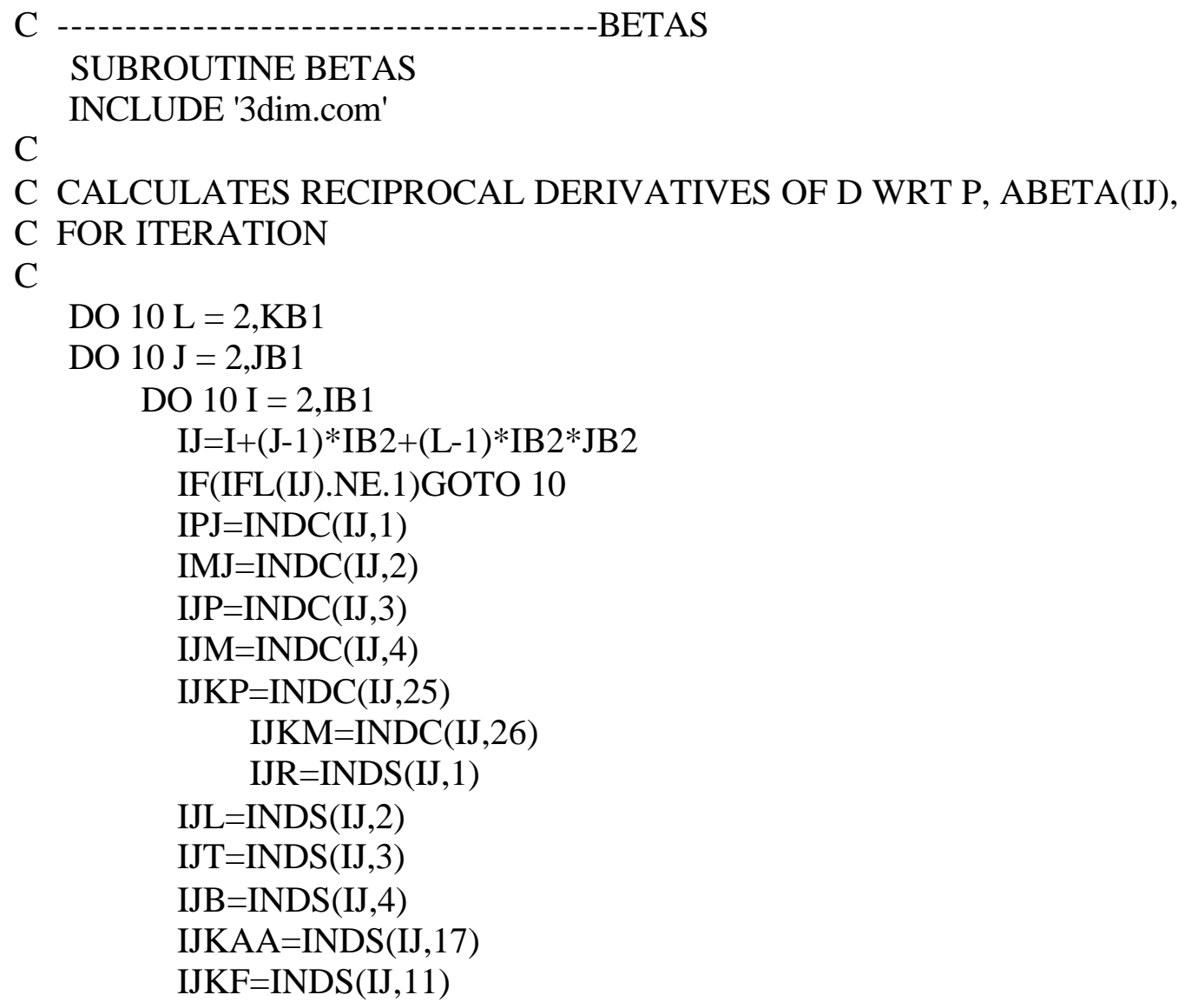




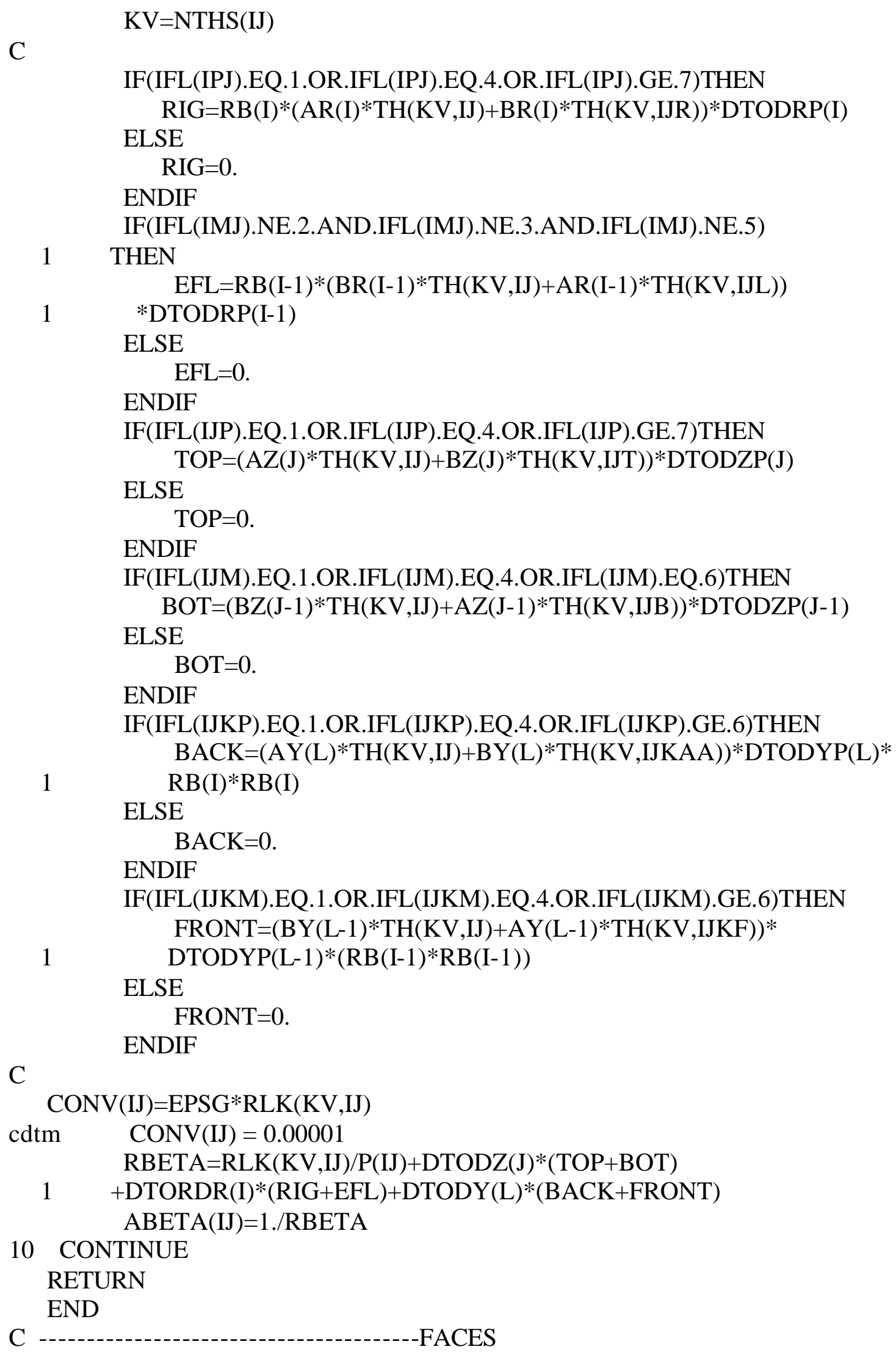




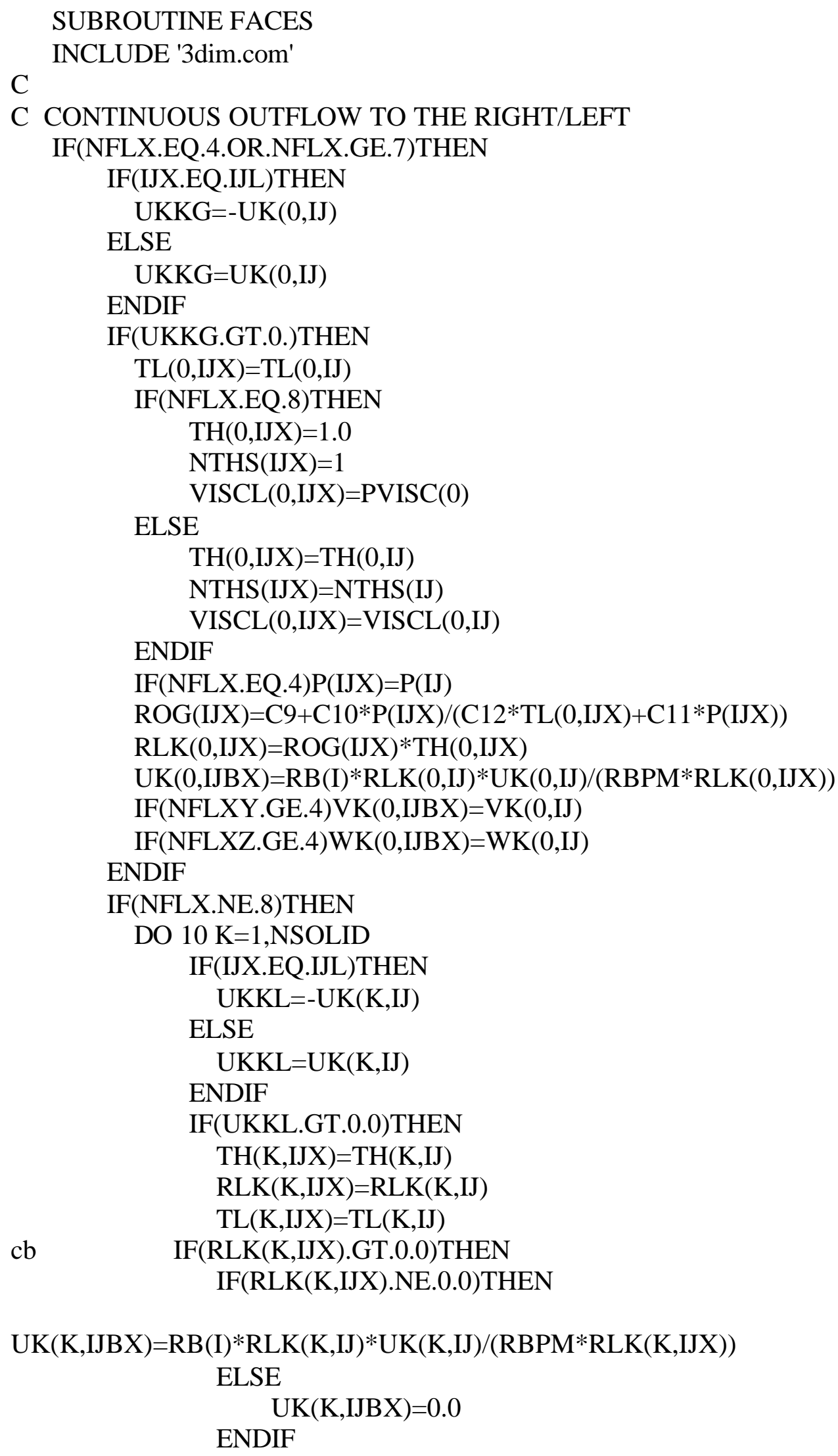




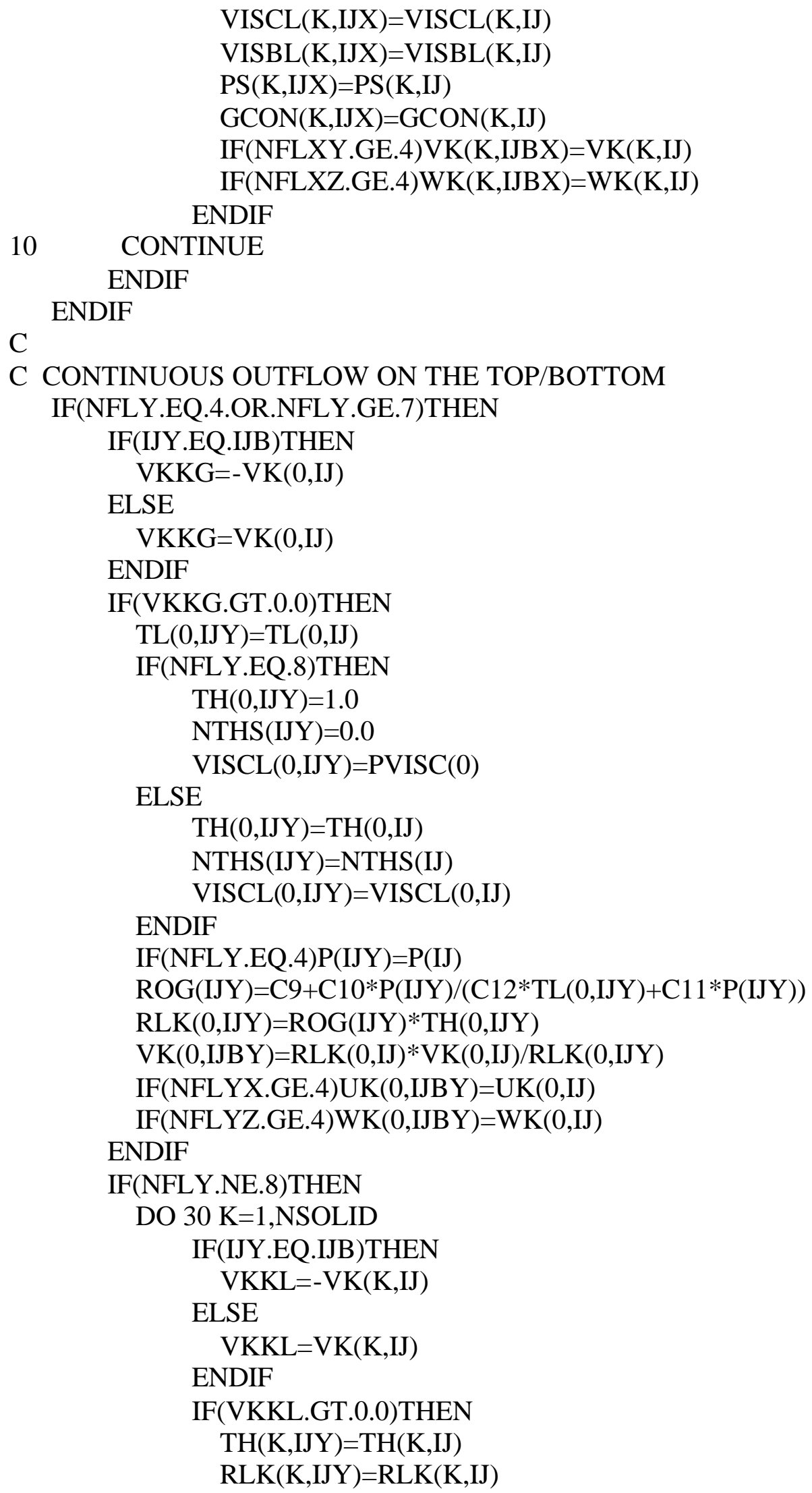




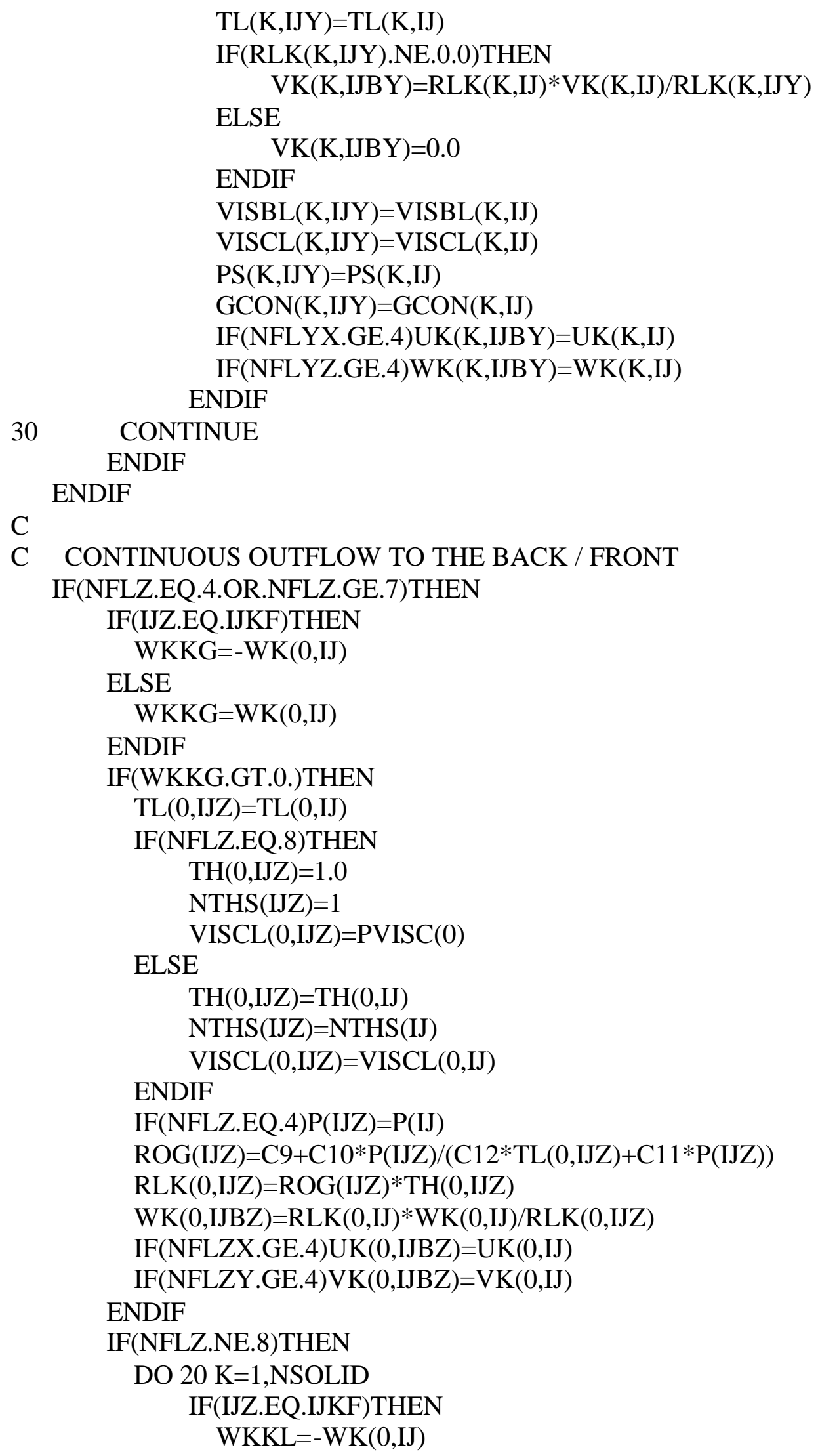




\section{ELSE}

$\mathrm{WKKL}=\mathrm{WK}(0, \mathrm{IJ})$

ENDIF

IF(WKKL.GT.0.)THEN

$\mathrm{TL}(\mathrm{K}, \mathrm{IJZ})=\mathrm{TL}(\mathrm{K}, \mathrm{IJ})$

$\mathrm{TH}(\mathrm{K}, \mathrm{IJZ})=\mathrm{TH}(\mathrm{K}, \mathrm{IJ})$

$\mathrm{RLK}(\mathrm{K}, \mathrm{IJZ})=\mathrm{RLK}(\mathrm{K}, \mathrm{IJ})$

IF(RLK(K,IJZ).NE.0.0)THEN

$\mathrm{WK}(\mathrm{K}, \mathrm{IJBZ})=\mathrm{RLK}(\mathrm{K}, \mathrm{IJ}) * \mathrm{WK}(\mathrm{K}, \mathrm{IJ}) / \mathrm{RLK}(\mathrm{K}, \mathrm{IJZ})$

ELSE

$\mathrm{WK}(\mathrm{K}, \mathrm{IJBZ})=0.0$

ENDIF

$\operatorname{VISCL}(\mathrm{K}, \mathrm{IJZ})=\mathrm{VISCL}(\mathrm{K}, \mathrm{IJ})$

VISBL $(K, I J Z)=V I S B L(K, I J)$

$\mathrm{PS}(\mathrm{K}, \mathrm{IJZ})=\mathrm{PS}(\mathrm{K}, \mathrm{IJ})$

$\operatorname{GCON}(\mathrm{K}, \mathrm{IJZ})=\mathrm{GCON}(\mathrm{K}, \mathrm{IJ})$

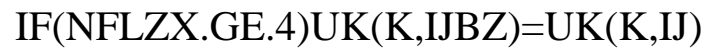

$\mathrm{IF}(\mathrm{NFLZY.GE.4)VK(K,IJBZ)=VK(K,IJ)}$

20

ENDIF

CONTINUE

ENDIF

ENDIF

C

C

C SET BOUNDARY CONDITIONS IN RIGID CELLS -

C GAS \& PARTICLE VELOCITIES; GRANULAR TEMPERATURES

$\mathrm{C}$

C FREE SLIP WALL ON THE RIGHT/LEFT

IF(NFLX .EQ. 2)THEN

IF(NFLXY.EQ.2.OR.NFLXY.EQ.3)THEN

DO $54 \mathrm{~K}=0$,NSOLID

$54 \quad \mathrm{VK}(\mathrm{K}, \mathrm{IJBX})=\mathrm{VK}(\mathrm{K}, \mathrm{IJ})$

ENDIF

IF(NFLXZ.EQ.2.OR.NFLXZ.EQ.3)THEN

DO $55 \mathrm{~K}=0$,NSOLID

$55 \quad \mathrm{WK}(\mathrm{K}, \mathrm{IJBX})=\mathrm{WK}(\mathrm{K}, \mathrm{IJ})$

ENDIF

C NO SLIP WALL ON THE RIGHT/LEFT

ELSEIF(NFLX.EQ.3)THEN

IF(NFLXZ.EQ.2.OR.NFLXZ.EQ.3)THEN

DO $56 \mathrm{~K}=0$,NCONT

$56 \quad \mathrm{WK}(\mathrm{K}, \mathrm{IJBX})=-\mathrm{WK}(\mathrm{K}, \mathrm{IJ})$

DO $57 \mathrm{~K}=\mathrm{NCONT}+1, \mathrm{NSOLID}$

$57 \mathrm{WK}(\mathrm{K}, \mathrm{IJBX})=\mathrm{WK}(\mathrm{K}, \mathrm{IJ}) *(\mathrm{PLP}(\mathrm{K})-\mathrm{DR}(\mathrm{I})) /(\mathrm{PLP}(\mathrm{K})+\mathrm{DR}(\mathrm{I}))$

ENDIF

IF(NFLXY.EQ.2.OR.NFLXY.EQ.3)THEN 


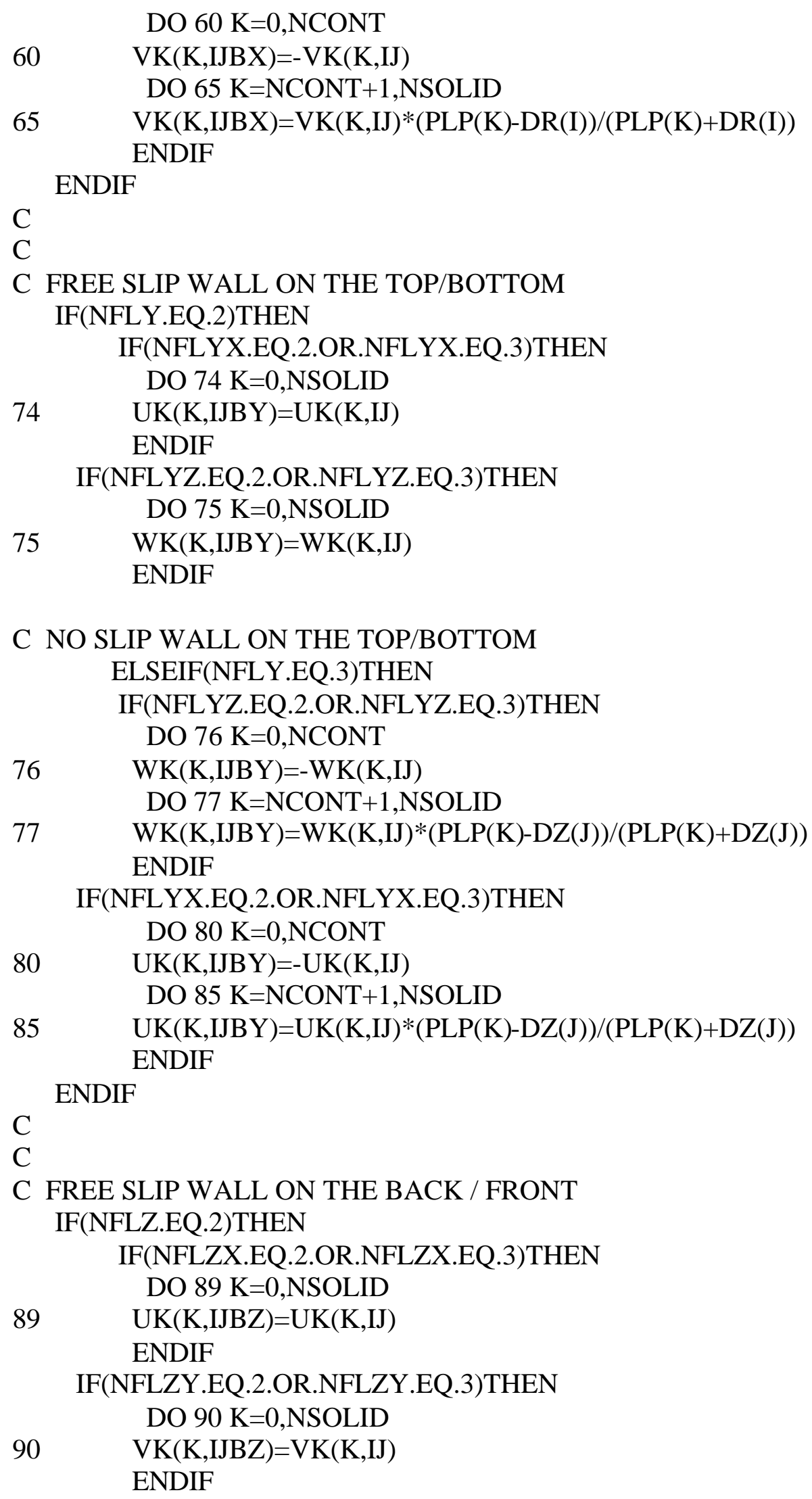

C FREE SLIP WALL ON THE BACK / FRONT IF(NFLZ.EQ.2)THEN IF(NFLZX.EQ.2.OR.NFLZX.EQ.3)THEN DO $89 \mathrm{~K}=0$,NSOLID

$89 \quad \mathrm{UK}(\mathrm{K}, \mathrm{IJBZ})=\mathrm{UK}(\mathrm{K}, \mathrm{IJ})$ ENDIF

IF(NFLZY.EQ.2.OR.NFLZY.EQ.3)THEN

DO $90 \mathrm{~K}=0$,NSOLID

$90 \quad \mathrm{VK}(\mathrm{K}, \mathrm{IJBZ})=\mathrm{VK}(\mathrm{K}, \mathrm{IJ})$

ENDIF 


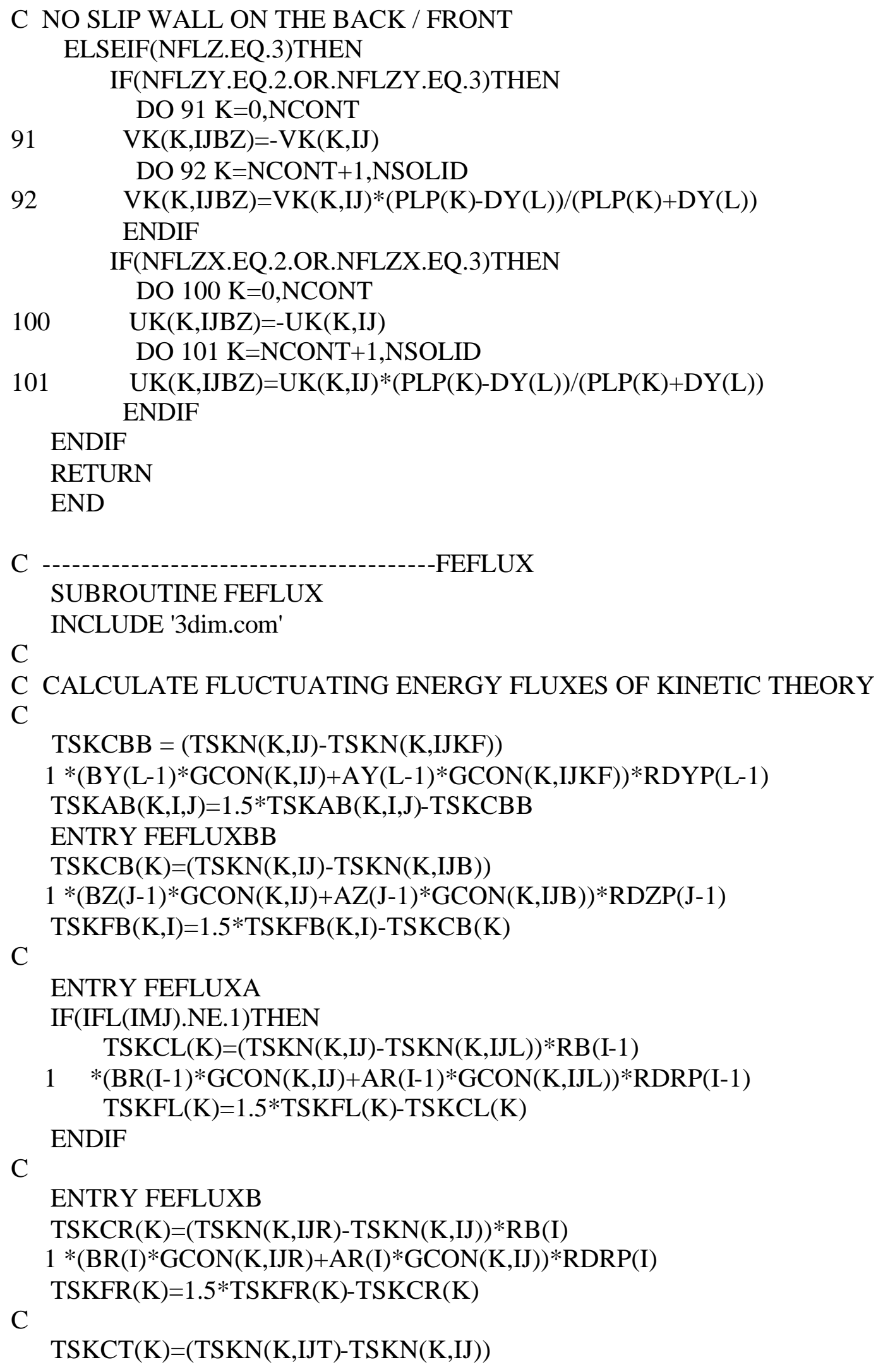




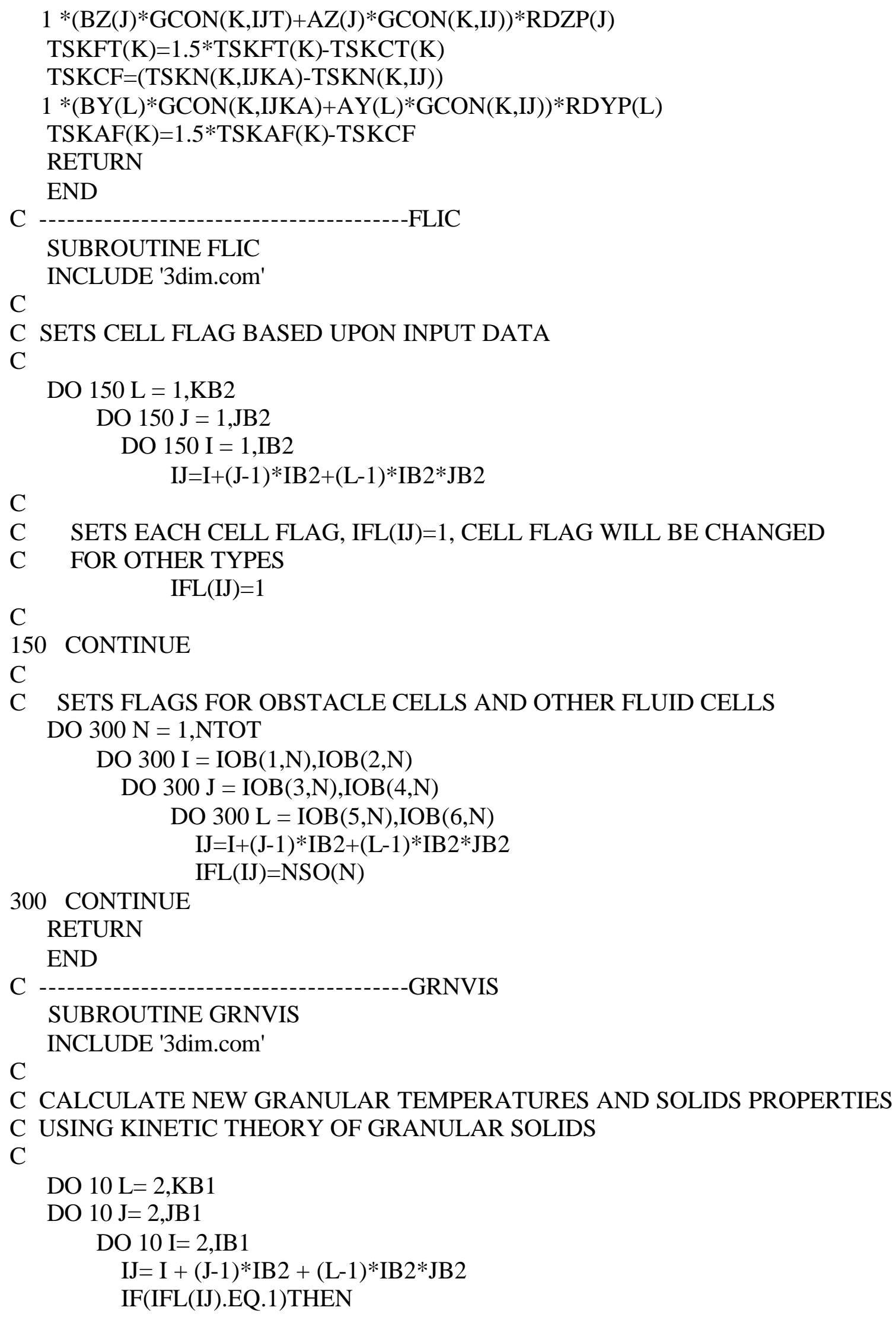




$$
\mathrm{IMJ}=\mathrm{INDC}(\mathrm{IJ}, 2)
$$$$
\mathrm{IJM}=\mathrm{INDC}(\mathrm{IJ}, 4)
$$$$
\mathrm{IJKM}=\operatorname{INDS}(\mathrm{IJ}, 15)
$$

C

$$
\mathrm{KV}=\mathrm{NTHS}(\mathrm{IJ})
$$

CALL KDRAGS(NCONT,NSOLID)

C

DO 5 K=NCONT,NSOLID

C GRANULAR KINETIC THEORY IS APPLIED

C ONLY IF (Ef-Ef,min) > 1.e-10 $\operatorname{IF}(\mathrm{RLK}(\mathrm{K}, \mathrm{IJ})$.GT.RLKMIN(K).AND.

C

$\begin{array}{ll}1 & (\mathrm{TH}(0, \mathrm{IJ})-\mathrm{THMIN}) . \mathrm{GT} .1 . \mathrm{E}-10) \mathrm{THEN} \\ \mathrm{TSKS}=\mathrm{TSK}(\mathrm{K}, \mathrm{IJ}) * * 0.5\end{array}$

C CALCULATE RADIAL DISTRIBUTION FUNCTION \& OTHER PARAMETERS

$\mathrm{G} 0=1.0 /\left(1.0-(\mathrm{TH}(\mathrm{K}, \mathrm{IJ}) /(1.0-\mathrm{THMIN}))^{* *}(1 . / 3).\right)$

$\mathrm{CS} 1=(1.0+\mathrm{CRES}) * \mathrm{TH}(\mathrm{K}, \mathrm{IJ}) * \mathrm{G} 0$

$\mathrm{CS} 2=3.0 *(1.0-\mathrm{CRES}) * \mathrm{RLK}(\mathrm{K}, \mathrm{IJ}) * \mathrm{CS} 1$

$\mathrm{CS} 3=\mathrm{RLK}(\mathrm{K}, \mathrm{IJ}) /(\mathrm{RLK}(\mathrm{K}, \mathrm{IJ})+\mathrm{DMFP}(\mathrm{K}))$

$\mathrm{CS} 4=2 * \mathrm{CS} 3 * \operatorname{VISDIL}(\mathrm{K}) /((1 .+\mathrm{CRES}) * \mathrm{G} 0)$

$\operatorname{GAMMA}(\mathrm{K}, \mathrm{IJ})=\mathrm{CS} 2 *(4.0 * \mathrm{RSQTP} / \mathrm{DK}(\mathrm{K}) * \mathrm{TSKS}-$

$1 \quad \operatorname{SILM}(\mathrm{K}, \mathrm{IJ}))$

$\mathrm{PS}(\mathrm{K}, \mathrm{IJ})=\mathrm{RLK}(\mathrm{K}, \mathrm{IJ}) *(1.0+2.0 * \mathrm{CS} 1)$

$\mathrm{C}$

1

VISCD $=\mathrm{CS} 4 *(1 .+.8 * \mathrm{CS} 1) * * 2$

$\operatorname{VISBL}(\mathrm{K}, \mathrm{IJ})=(4 . / 3). * \mathrm{RLK}(\mathrm{K}, \mathrm{IJ}) * \mathrm{DK}(\mathrm{K}) * \mathrm{CS} 1 *$

RSQTP*CS3

$\operatorname{VISCL}(\mathrm{K}, \mathrm{IJ})=\mathrm{VISCD}+0.6 * \mathrm{VISBL}(\mathrm{K}, \mathrm{IJ})$

$\mathrm{C}$

C GRANULAR CONDUCTIVITY

$\mathrm{GCON}(\mathrm{K}, \mathrm{IJ})=3.75 * \mathrm{CS} 4 *(1 .+1.2 * \mathrm{CS} 1) * * 2 * \mathrm{TSKS}+$

1

$1.5 * \operatorname{VISBL}(\mathrm{K}, \mathrm{IJ})$

ELSE

$$
\operatorname{GCON}(\mathrm{K}, \mathrm{IJ})=0.0
$$

5 ENDIF

5 CONTINUE ENDIF

10 CONTINUE

$\mathrm{C}$

C FINAL SOLUTION OF GRANULAR TEMPERATURE

DO $100 \mathrm{~L}=2, \mathrm{~KB} 1$

DO $100 \mathrm{~J}=2, \mathrm{JB} 1$

DO $100 \mathrm{I}=2, \mathrm{IB} 1$

$\mathrm{IJ}=\mathrm{I}+(\mathrm{J}-1) * \mathrm{IB} 2+(\mathrm{L}-1) * \mathrm{IB} 2 * \mathrm{JB} 2$

IF(IFL(IJ).EQ.1)THEN

$\mathrm{IMJ}=\mathrm{INDC}(\mathrm{IJ}, 2)$ 


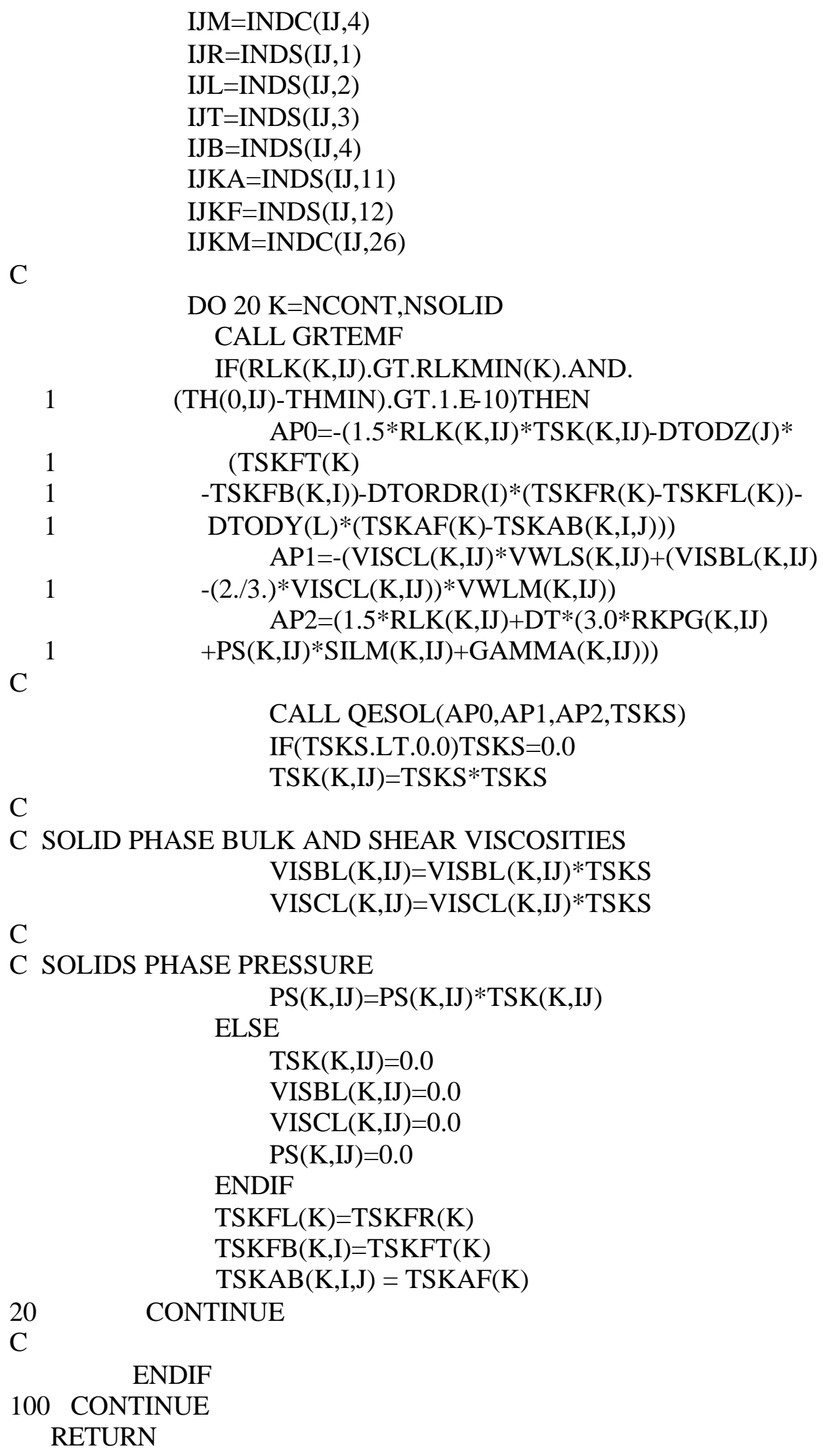




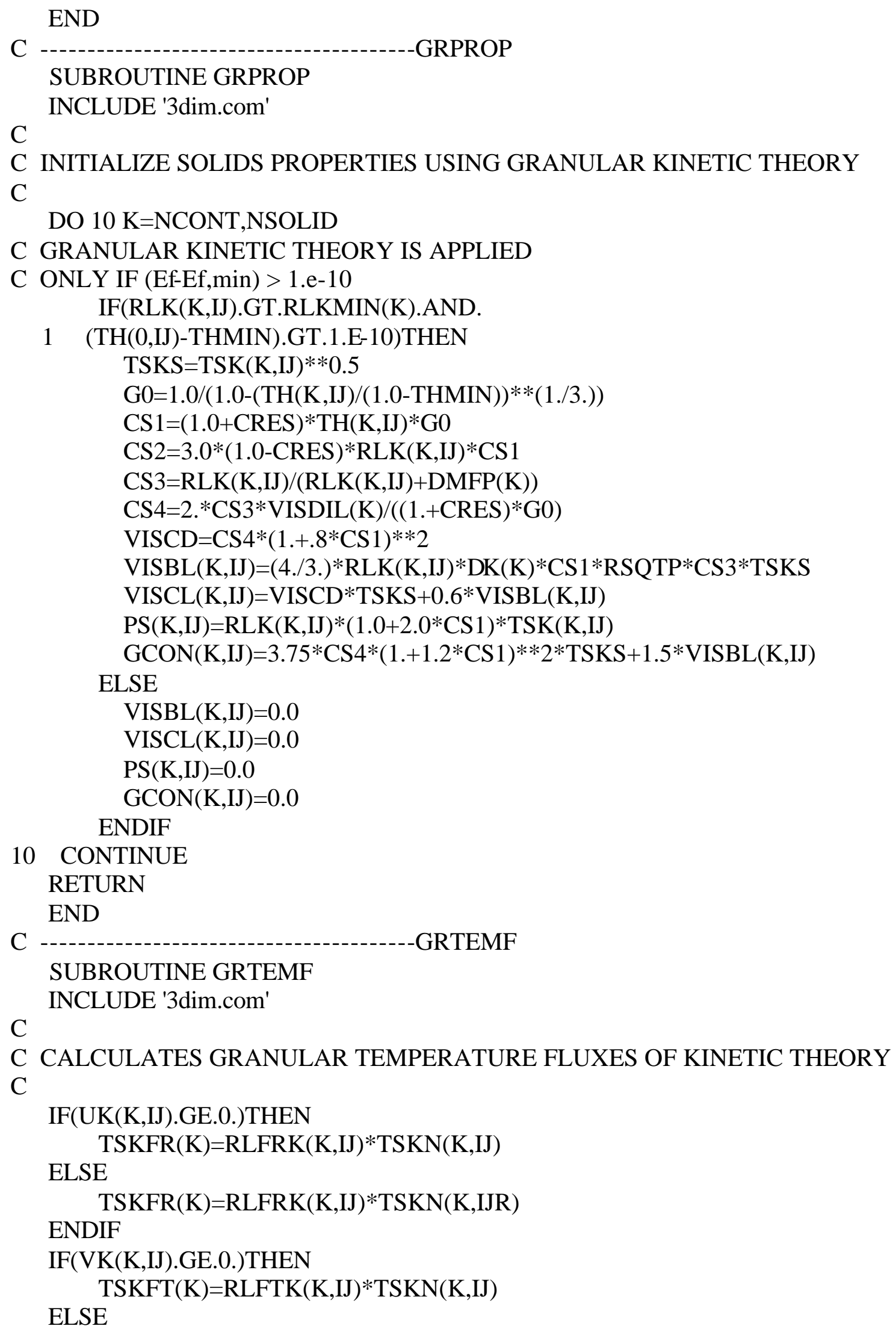




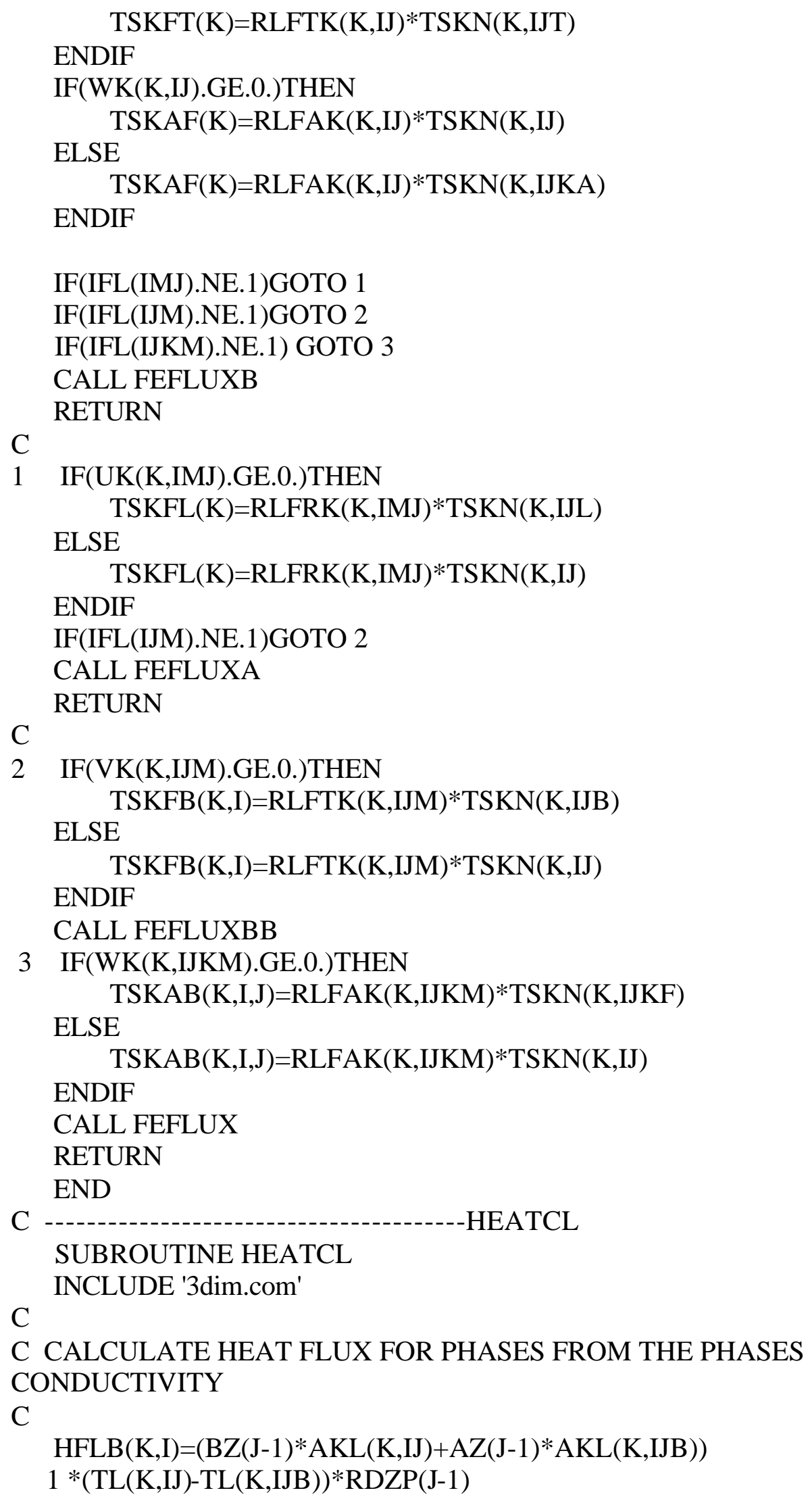




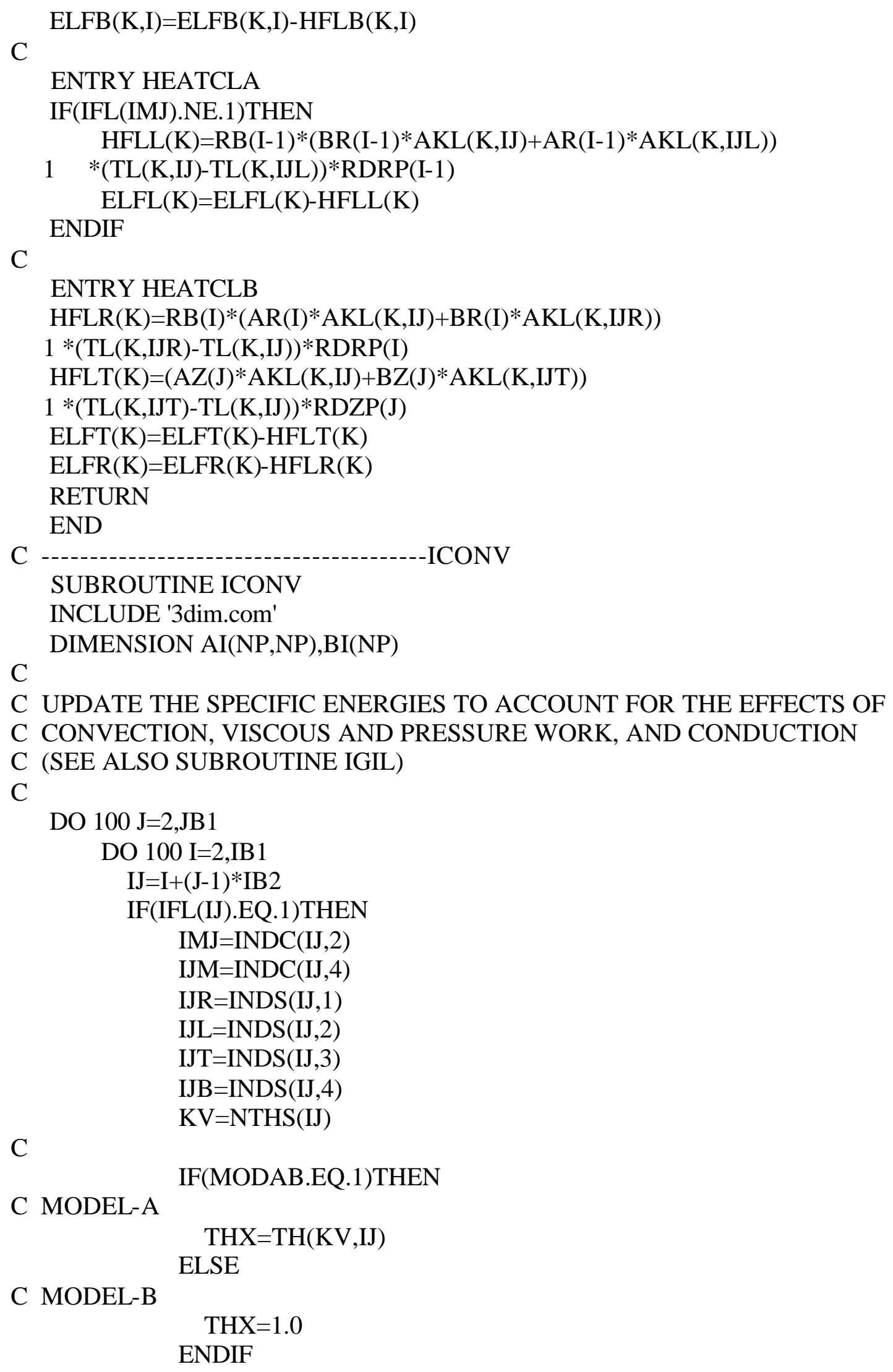




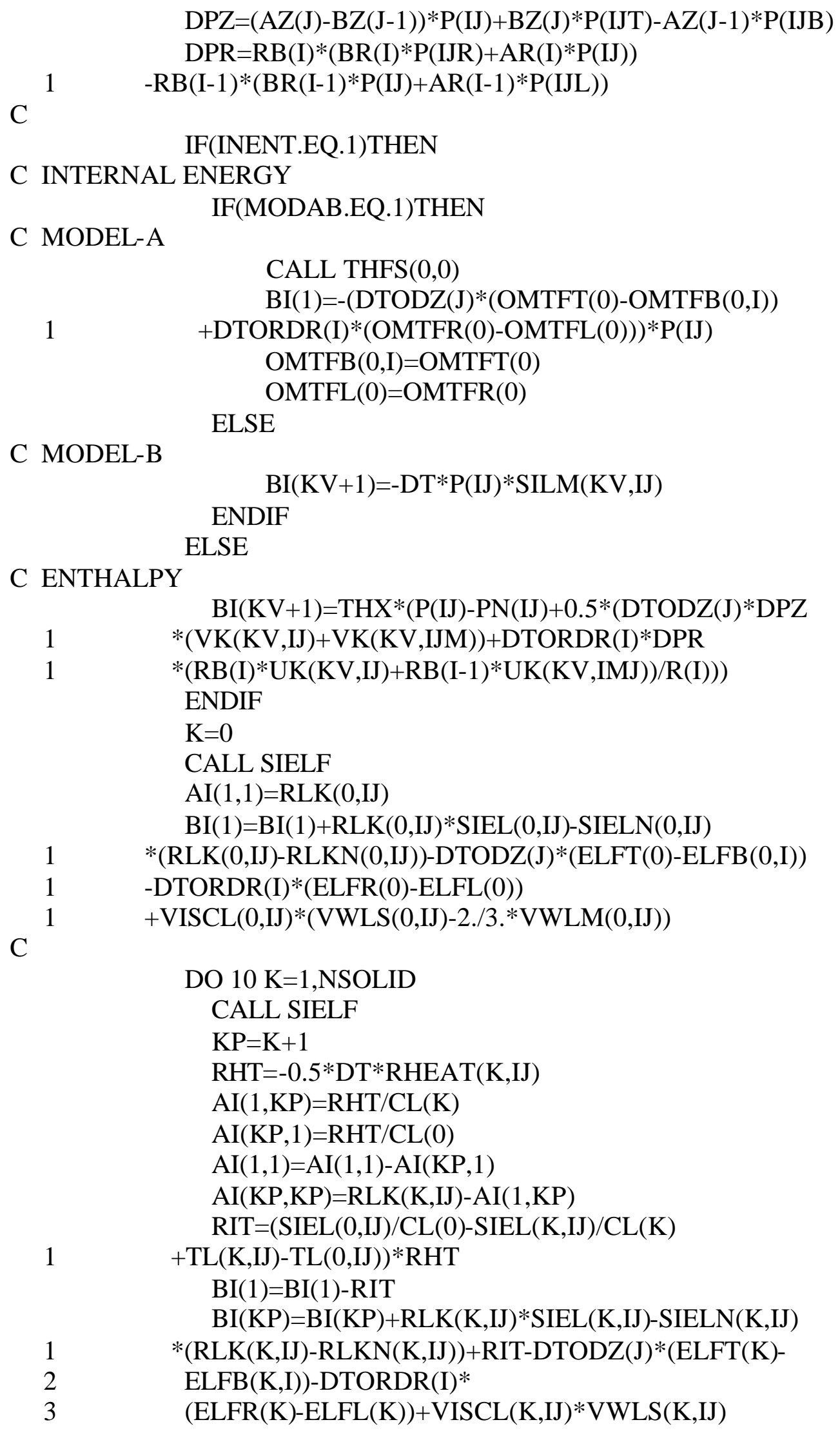




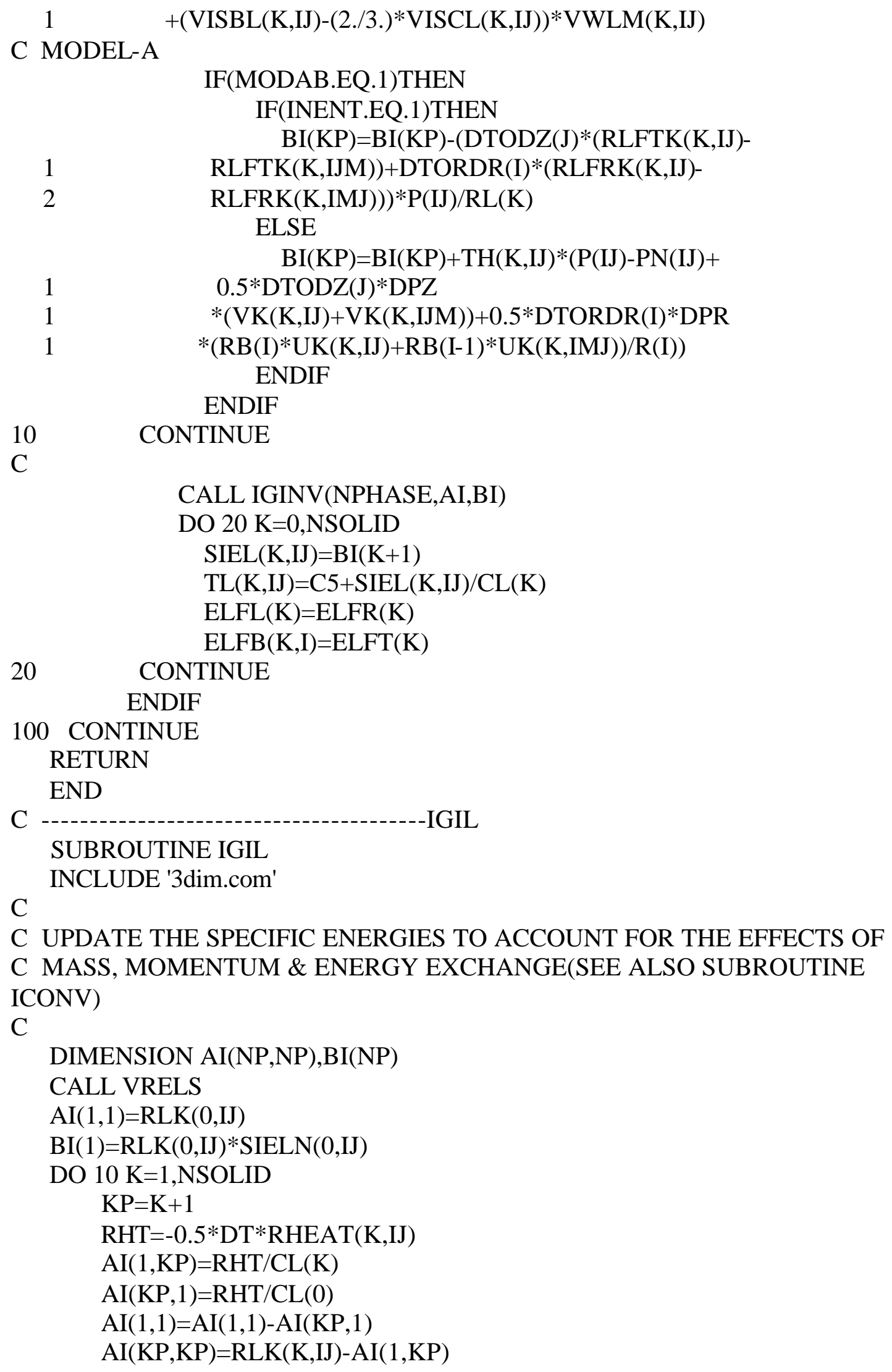

C UPDATE THE SPECIFIC ENERGIES TO ACCOUNT FOR THE EFFECTS OF C MASS, MOMENTUM \& ENERGY EXCHANGE(SEE ALSO SUBROUTINE ICONV)

$\mathrm{C}$

DIMENSION AI(NP,NP),BI(NP)

CALL VRELS

$\operatorname{AI}(1,1)=\operatorname{RLK}(0, \mathrm{IJ})$

$\operatorname{BI}(1)=\operatorname{RLK}(0, \mathrm{IJ}) * \operatorname{SIELN}(0, \mathrm{IJ})$

DO $10 \mathrm{~K}=1$,NSOLID

$\mathrm{KP}=\mathrm{K}+1$

RHT $=-0.5 * \mathrm{DT} *$ RHEAT $(\mathrm{K}, \mathrm{IJ})$

$\mathrm{AI}(1, \mathrm{KP})=\mathrm{RHT} / \mathrm{CL}(\mathrm{K})$

$\mathrm{AI}(\mathrm{KP}, 1)=\mathrm{RHT} / \mathrm{CL}(0)$

$\mathrm{AI}(1,1)=\mathrm{AI}(1,1)-\mathrm{AI}(\mathrm{KP}, 1)$

$\mathrm{AI}(\mathrm{KP}, \mathrm{KP})=\mathrm{RLK}(\mathrm{K}, \mathrm{IJ})-\mathrm{AI}(1, \mathrm{KP})$ 


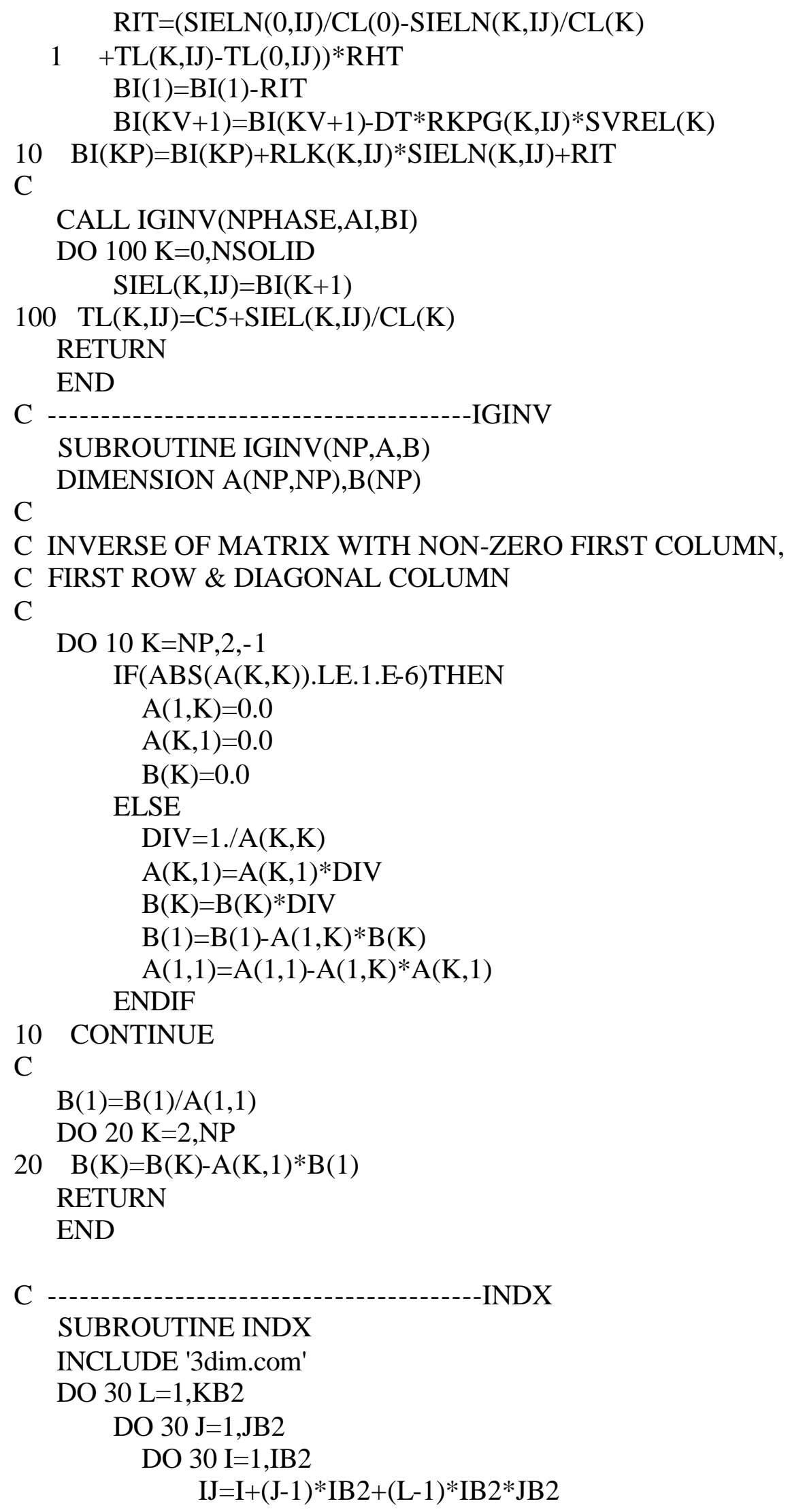




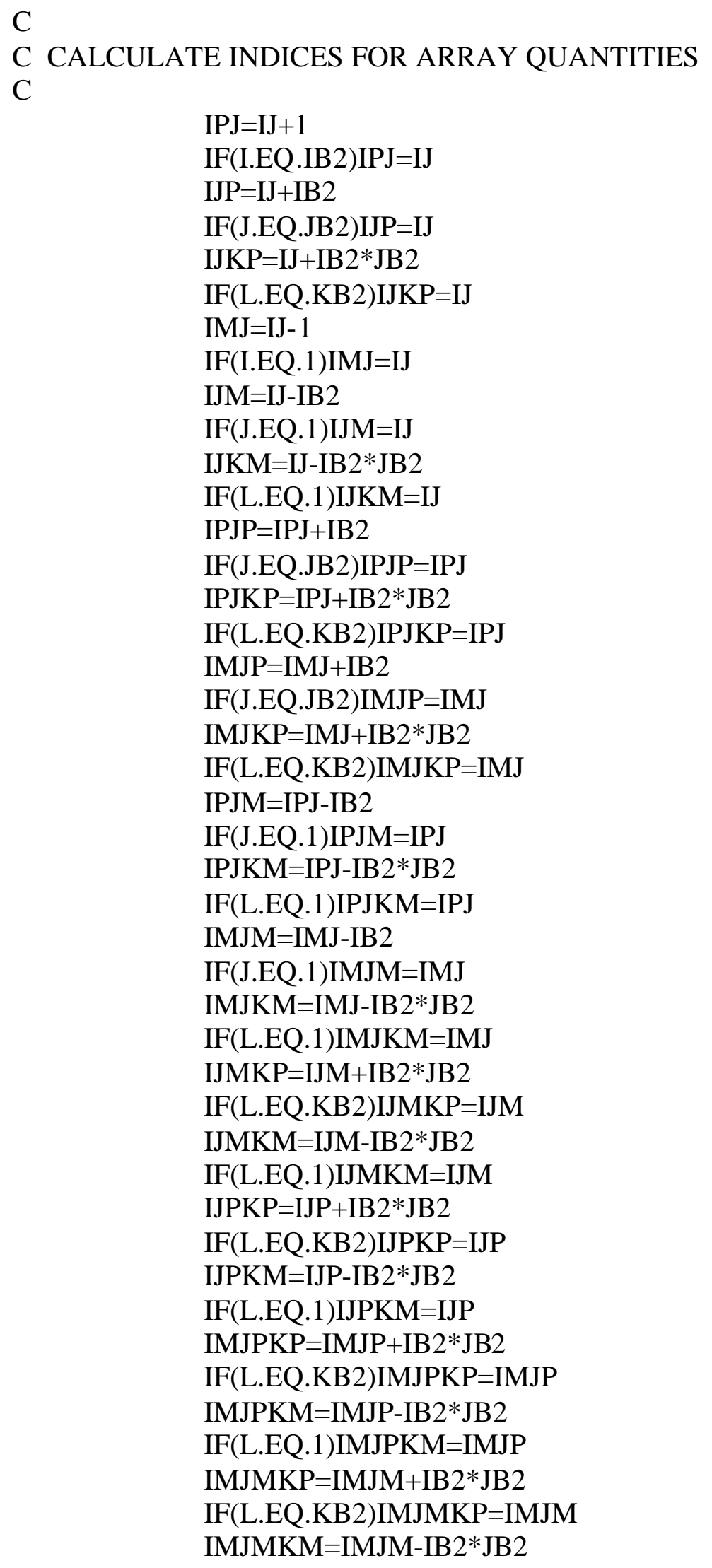



IF(L.EQ.1)IMJMKM=IMJM
IPJPKP=IPJP+IB2*JB2
IF(L.EQ.KB2)IPJPKP=IPJP
IPJPKM=IPJP-IB2*JB2
IF(L.EQ.1)IPJPKM=IPJP
IPJMKP=IPJM+IB2*JB2
IF(L.EQ.KB2)IPJMKP=IPJM
IPJMKM=IPJM-IB $2 * J B 2$
IF(L.EQ.1)IPJMKM=IPJM

C

$$
\begin{aligned}
& \text { INDC(IJ,1)=IPJ } \\
& \text { INDC(IJ,2)=IMJ } \\
& \text { INDC(IJ,3)=IJP } \\
& \text { INDC(IJ,4)=IJM } \\
& \text { INDC(IJ,5)=IPJP } \\
& \text { INDC(IJ,6)=IMJP } \\
& \text { INDC(IJ,7)=IPJM } \\
& \text { INDC(IJ,8)=IMJM } \\
& \text { INDC(IJ,9)=IPJKP } \\
& \text { INDC(IJ,10)=IPJKM } \\
& \text { INDC(IJ,11)=IMJKM } \\
& \text { INDC(IJ,12)=IJMKP } \\
& \text { INDC(IJ,13)=IJPKP } \\
& \text { INDC(IJ,14)=IJMKM } \\
& \text { INDC(IJ,15)=IJPKM } \\
& \text { INDC(IJ,16)=IMJPKP } \\
& \text { INDC(IJ,17)=IMJPKM } \\
& \text { INDC(IJ,18)=IMJMKP } \\
& \text { INDC(IJ,19)=IMJMKM } \\
& \text { INDC(IJ,20)=IPJPKP } \\
& \text { INDC(IJ,21)=IPJPKM } \\
& \text { INDC(IJ,22)=IPJMKP } \\
& \text { INDC(IJ,23)=IPJMKM } \\
& \text { INDC(IJ,24)=IMJKP } \\
& \text { INDC(IJ,25)=IJKP } \\
& \text { INDC(IJ,26)=IJKM }
\end{aligned}
$$

C

C INITIALIZE 'INDC'

$$
\begin{aligned}
& \text { IJR=IPJ } \\
& \text { IF(IFL(IPJ).EQ.2.OR.IFL(IPJ).EQ.3)IJR=IJ } \\
& \text { IJL=IMJ } \\
& \text { IF(IFL(IMJ).EQ.2.OR.IFL(IMJ).EQ.3)IJL=IJ } \\
& \text { IJT=IJP } \\
& \text { IF(IFL(IJP).EQ.2.OR.IFL(IJP).EQ.3)IJT=IJ } \\
& \text { IJB=IJM }
\end{aligned}
$$




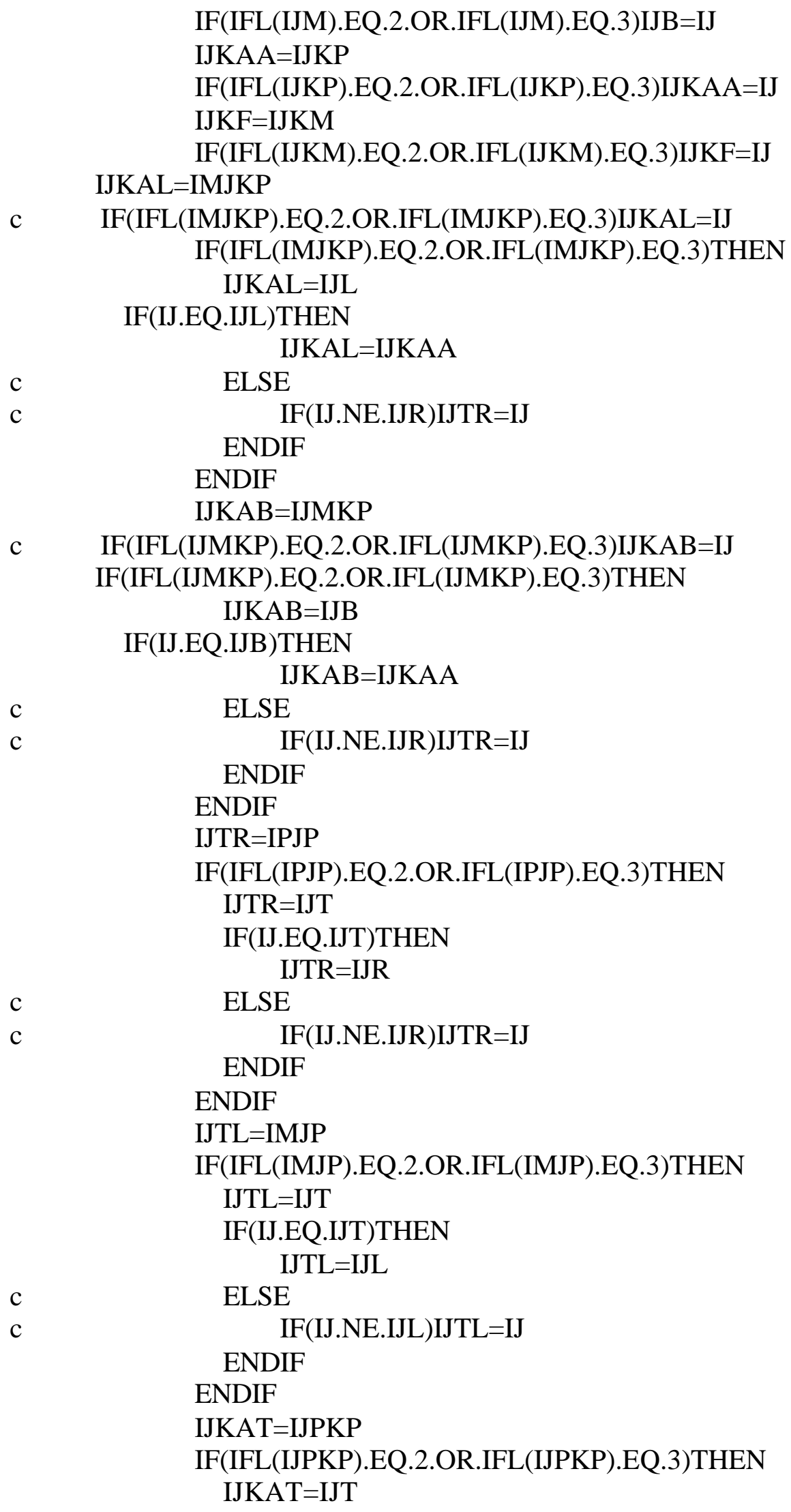


c

c

c

c

c

c

c

c

c

c

c

C

C

C

C

C

C

C

C

C

$$
\begin{array}{r}
\text { IF(IJ.EQ.IJT)THEN } \\
\text { IJKAT=IJKAA }
\end{array}
$$

ELSE

IF(IJ.NE.IJKAA)IJKAT $=$ IJ

ENDIF

\section{ENDIF}

IJKFT $=I J P K M$

IF(IFL(IJPKM).EQ.2.OR.IFL(IJPKM).EQ.3)THEN

$\mathrm{IJKFT}=\mathrm{IJT}$

IF(IJ.EQ.IJT)THEN

$\mathrm{IJKFT}=\mathrm{IJKF}$

ELSE

IF(IJ.NE.IJKF)IJKFT=IJ

ENDIF

\section{ENDIF}

$\mathrm{IJBR}=\mathrm{IPJM}$

IF(IFL(IPJM).EQ.2.OR.IFL(IPJM).EQ.3)THEN

$\mathrm{IJBR}=\mathrm{IJB}$

IF(IJ.EQ.IJB)THEN

IJBR=IJR

ELSE

IF(IJ.NE.IJR)IJBR=IJ

ENDIF

ENDIF

IJKAR=IPJKP

IF(IFL(IPJKP).EQ.2.OR.IFL(IPJKP).EQ.3)THEN

IJKAR $=$ IJKAA

IF(IJ.EQ.IJKAA)THEN

IJKAR $=$ IJR

$\mathrm{IJKAR}=\mathrm{IJR}$

IF(IJ.EQ.IJR)THEN

IJKAR=IJKAA

ELSE

IF(IJ.NE.IJR)IJKAR=IJ

ENDIF

$$
\text { ENDIF }
$$

$\mathrm{IJPKP}=\mathrm{IJP}+\mathrm{IB} 2 * \mathrm{JB} 2$

IJKAZ=IJPKP

IF(IFL(IJPKP).EQ.2.OR.IFL(IJPKP).EQ.3)THEN

IJKAR=IJT

IF(IJ.EQ.IJT)THEN

$\mathrm{IJKAR}=\mathrm{IJKP}$

ELSE

IF(IJ.NE.IJKP)IJKAZ=IJ

ENDIF 


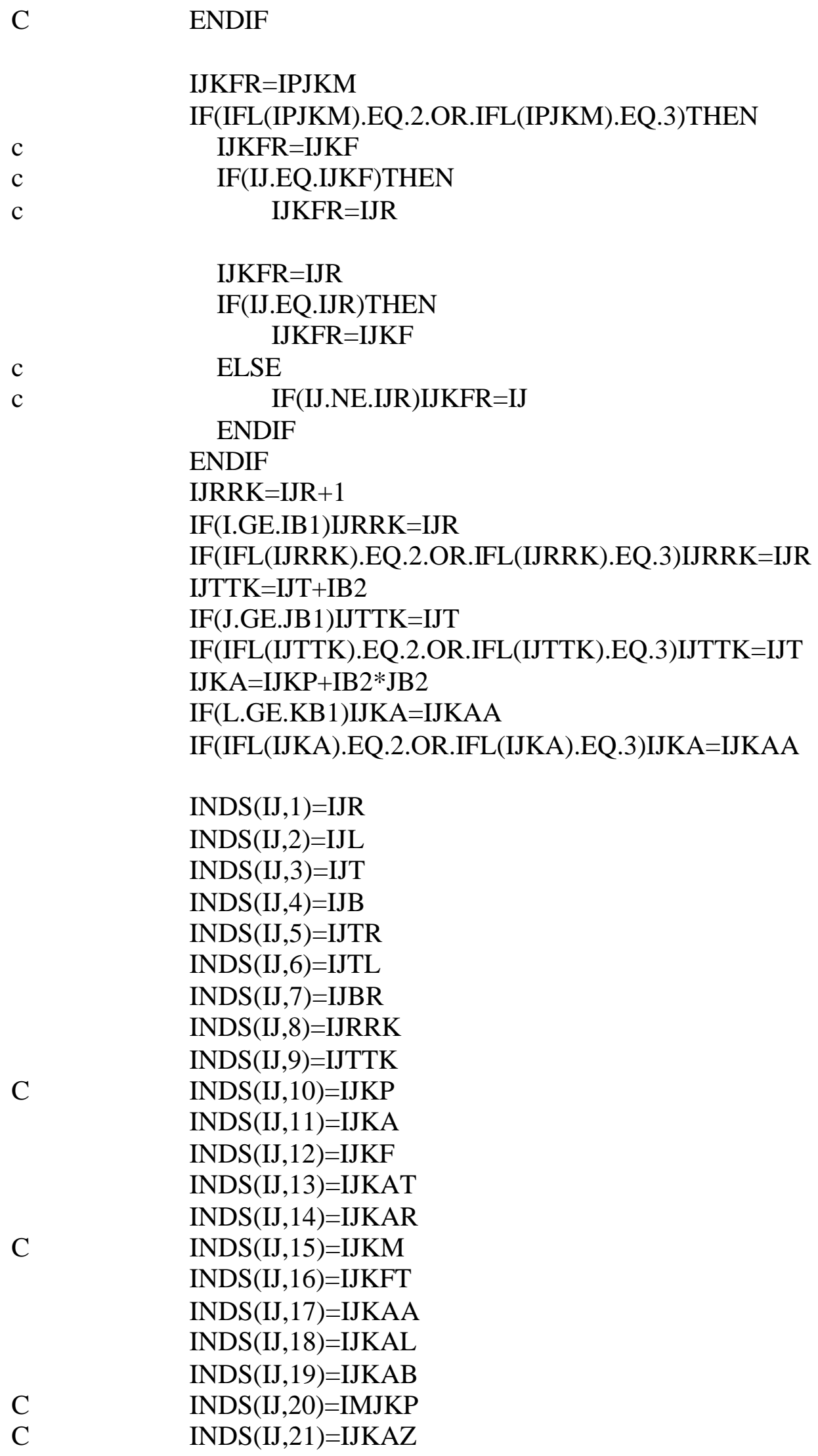


$\operatorname{INDS}(\mathrm{IJ}, 22)=\mathrm{IJKFR}$

30 CONTINUE

RETURN

END

$\mathrm{C}$

SUBROUTINE ITER

-ITER

$\mathrm{C}$

INCLUDE '3dim.com'

C PERFORM THE ITERATIVE SOLUTION OF DIFFERENCE EQUATIONS OF

C MASS, MOMENTUM AND ENERGY EQUATIONS

$\mathrm{C}$

LOGICAL MUSTIT

PARAMETER (NTMAX $=400$, LMAX $=5$, OMEGA $=1.0$ )

MUSTIT $=$.FALSE.

DO 200 NIT=1,NTMAX

NITER $($ ITX $)=N$ ITER $($ ITX $)+1$

DO $100 \mathrm{~L}=2, \mathrm{~KB} 1$

DO $100 \mathrm{~J}=2, \mathrm{JB} 1$

DO $100 \mathrm{I}=2, \mathrm{IB} 1$

$\mathrm{IJ}=\mathrm{I}+(\mathrm{J}-1) * \mathrm{IB} 2+(\mathrm{L}-1) * \mathrm{IB} 2 * \mathrm{JB} 2$

IF(IFL(IJ).NE.1)GOTO 100

LOOP $=0$

$\mathrm{KROS}=-1$

$\mathrm{KV}=\mathrm{NTHS}(\mathrm{IJ})$

$\mathrm{IPJ}=\mathrm{INDC}(\mathrm{IJ}, 1)$

$\mathrm{IMJ}=\mathrm{INDC}(\mathrm{IJ}, 2)$

$\mathrm{IJP}=\mathrm{INDC}(\mathrm{IJ}, 3)$

$\mathrm{IJM}=\mathrm{INDC}(\mathrm{IJ}, 4)$

$\mathrm{IJKP}=\mathrm{INDC}(\mathrm{IJ}, 25)$

$\mathrm{IJKM}=\mathrm{INDC}(\mathrm{IJ}, 26)$

$\mathrm{IJR}=\mathrm{INDS}(\mathrm{IJ}, 1)$

$\mathrm{IJL}=\mathrm{INDS}(\mathrm{IJ}, 2)$

$\mathrm{IJT}=\mathrm{INDS}(\mathrm{IJ}, 3)$

$\mathrm{IJB}=\mathrm{INDS}(\mathrm{IJ}, 4)$

IJKAA $=\operatorname{INDS}(\mathrm{IJ}, 17)$

$\mathrm{IJKF}=\mathrm{INDS}(\mathrm{IJ}, 12)$

$\mathrm{DG}=\mathrm{RLK}(\mathrm{KV}, \mathrm{IJ})-\mathrm{RLKN}(\mathrm{KV}, \mathrm{IJ})+\mathrm{DTORDR}(\mathrm{I}) *(\mathrm{RLFRK}(\mathrm{KV}, \mathrm{IJ})$

1 $-\operatorname{RLFRK}(\mathrm{KV}, \mathrm{IMJ}))+\mathrm{DTODZ}(\mathrm{J}) *(\operatorname{RLFTK}(\mathrm{KV}, \mathrm{IJ})-$

$\operatorname{RLFTK}(\mathrm{KV}, \mathrm{IJM}))$

$+(\operatorname{RLFAK}(\mathrm{KV}, \mathrm{IJ})-\mathrm{RLFAK}(\mathrm{KV}, \mathrm{IJKM})) * \operatorname{DTODY}(\mathrm{L}) / \mathrm{R}(\mathrm{I})$

TARGET $=(1.0-$ OMEGA $) *$ DG

$\mathrm{ADG}=\mathrm{ABS}(\mathrm{DG})$

ADGTAR $=$ ABS $($ DG-TARGET $)$

DGORIG=ADG 
IF(ADG.LE.CONV(IJ))GOTO 78

MUSTIT=.FALSE.

D3=DG

$\mathrm{P} 3=\mathrm{P}(\mathrm{IJ})$

IF(NIT.EQ.1)GOTO 55

10

IF(D3.GT.TARGET)GOTO 11

$\mathrm{D} 2=\mathrm{D} 3$

$\mathrm{P} 2=\mathrm{P} 3$

IF(KROS.EQ.-1)KROS=1

IF(KROS.EQ.0)KROS=2

GOTO 12

$11 \quad$ D1=D3

$\mathrm{P} 1=\mathrm{P} 3$

IF(KROS.EQ.- 1$)$ KROS $=0$

IF(KROS.EQ.1)KROS $=2$

12

IF(KROS.EQ.3)GOTO 54

IF(KROS.EQ.2)GOTO 13

D3TAR=D3-TARGET

$\mathrm{DP}=-\mathrm{D} 3 \mathrm{TAR} * \mathrm{ABETA}(\mathrm{IJ})$

DSN=SIGN(1.,D3TAR)

IF(-DP*DSN.GT.0.25*P3)DP=-0.5*DSN*P3

$\mathrm{P}(\mathrm{IJ})=\mathrm{P}(\mathrm{IJ})+\mathrm{DP}$

GOTO 54

13

$\mathrm{P}(\mathrm{IJ})=(\mathrm{D} 1 * \mathrm{P} 2-\mathrm{D} 2 * \mathrm{P} 1+\mathrm{TARGET} *(\mathrm{P} 1-\mathrm{P} 2)) /(\mathrm{D} 1-\mathrm{D} 2)$

$\operatorname{ABETA}(\mathrm{IJ})=(\mathrm{P} 1-\mathrm{P} 2) /(\mathrm{D} 1-\mathrm{D} 2)$

$\mathrm{KROS}=3$

54

$\mathrm{P} 3=\mathrm{P}(\mathrm{IJ})$

$55 \quad$ CONTINUE

IF(INENT.NE.0)CALL IGIL

$\mathrm{ROG}(\mathrm{IJ})=\mathrm{C} 9+\mathrm{C} 10 * \mathrm{P}(\mathrm{IJ}) /(\mathrm{C} 12 * \mathrm{TL}(0, \mathrm{IJ})+\mathrm{C} 11 * \mathrm{P}(\mathrm{IJ}))$

$\operatorname{RLK}(0, \mathrm{IJ})=\mathrm{TH}(0, \mathrm{IJ}) * \mathrm{ROG}(\mathrm{IJ})$

CALL MATS

CALL VELSK

CALL MASFK $(0, \mathrm{KV}-1)$

CALL MASFK $(\mathrm{KV}+1, \mathrm{NSOLID})$

78 THX $=0.0$

DO $79 \mathrm{~K}=0$,NSOLID

IF(K.NE.KV)THEN

$\mathrm{RLK}(\mathrm{K}, \mathrm{IJ})=\mathrm{RLKN}(\mathrm{K}, \mathrm{IJ})-\mathrm{DTORDR}(\mathrm{I}) *(\operatorname{RLFRK}(\mathrm{K}, \mathrm{IJ})$

$-\operatorname{RLFRK}(\mathrm{K}, \mathrm{IMJ}))$-DTODZ(J)*(RLFTK(K,IJ)-

$\operatorname{RLFTK}(\mathrm{K}, \mathrm{IJM}))$

-(RLFAK(K,IJ)-RLFAK(K,IJKM))*DTODY(L)/R(I)

$\operatorname{IF}(\mathrm{RLK}(\mathrm{K}, \mathrm{IJ})$.LT.1.E-6*RLKMIN(K))RLK(K,IJ) $=0.0$

IF(K.EQ.0)THEN

$\operatorname{IF}(\operatorname{RLK}(0, \mathrm{IJ}) \cdot \mathrm{GT} \cdot \mathrm{ROG}(\mathrm{IJ})) \mathrm{RLK}(0, \mathrm{IJ})=\mathrm{ROG}(\mathrm{IJ})$

$\mathrm{TH}(0, \mathrm{IJ})=\mathrm{RLK}(0, \mathrm{IJ}) / \mathrm{ROG}(\mathrm{IJ})$ 


\section{ELSE}

$\operatorname{IF}(\mathrm{RLK}(\mathrm{K}, \mathrm{IJ}) . \mathrm{GT} \cdot \mathrm{RL}(\mathrm{K})) \mathrm{RLK}(\mathrm{K}, \mathrm{IJ})=\mathrm{RL}(\mathrm{K})$

$\mathrm{TH}(\mathrm{K}, \mathrm{IJ})=\mathrm{RLK}(\mathrm{K}, \mathrm{IJ}) / \mathrm{RL}(\mathrm{K})$

\section{ENDIF}

$\mathrm{THX}=\mathrm{THX}+\mathrm{TH}(\mathrm{K}, \mathrm{IJ})$

\section{ENDIF}

$\mathrm{c}$

CONTINUE

IF(THX.GT.0.8)THEN

$\mathrm{DIV}=0.8 / \mathrm{THX}$

THX $=0.8$

DO $81 \mathrm{~K}=1$,NSOLID

$\mathrm{RLK}(\mathrm{K}, \mathrm{IJ})=\mathrm{RLK}(\mathrm{K}, \mathrm{IJ}) * \mathrm{DIV}$

CONTINUE

ENDIF

$\mathrm{TH}(\mathrm{KV}, \mathrm{IJ})=1 .-\mathrm{THX}$

IF(KV.EQ.0)THEN

$\mathrm{RLK}(0, \mathrm{IJ})=\mathrm{TH}(0, \mathrm{IJ}) * \mathrm{ROG}(\mathrm{IJ})$

ELSE

$\mathrm{RLK}(\mathrm{KV}, \mathrm{IJ})=\mathrm{TH}(\mathrm{KV}, \mathrm{IJ}) * \mathrm{RL}(\mathrm{KV})$

ENDIF

IF(ADGTAR.LE.CONV(IJ))GOTO 100

CALL MASFK(KV,KV)

$\mathrm{DG}=\mathrm{RLK}(\mathrm{KV}, \mathrm{IJ})-\mathrm{RLKN}(\mathrm{KV}, \mathrm{IJ})+\mathrm{DTORDR}(\mathrm{I}) *(\mathrm{RLFRK}(\mathrm{KV}, \mathrm{IJ})$

100

CONTINUE

IF(MUSTIT)RETURN

MUSTIT $=$.TRUE.

IF(NIT.EQ.NTMAX)THEN

WRITE( $(21, *)$ 'MAX ITERATION AT TIME = ',TIME,NIT 


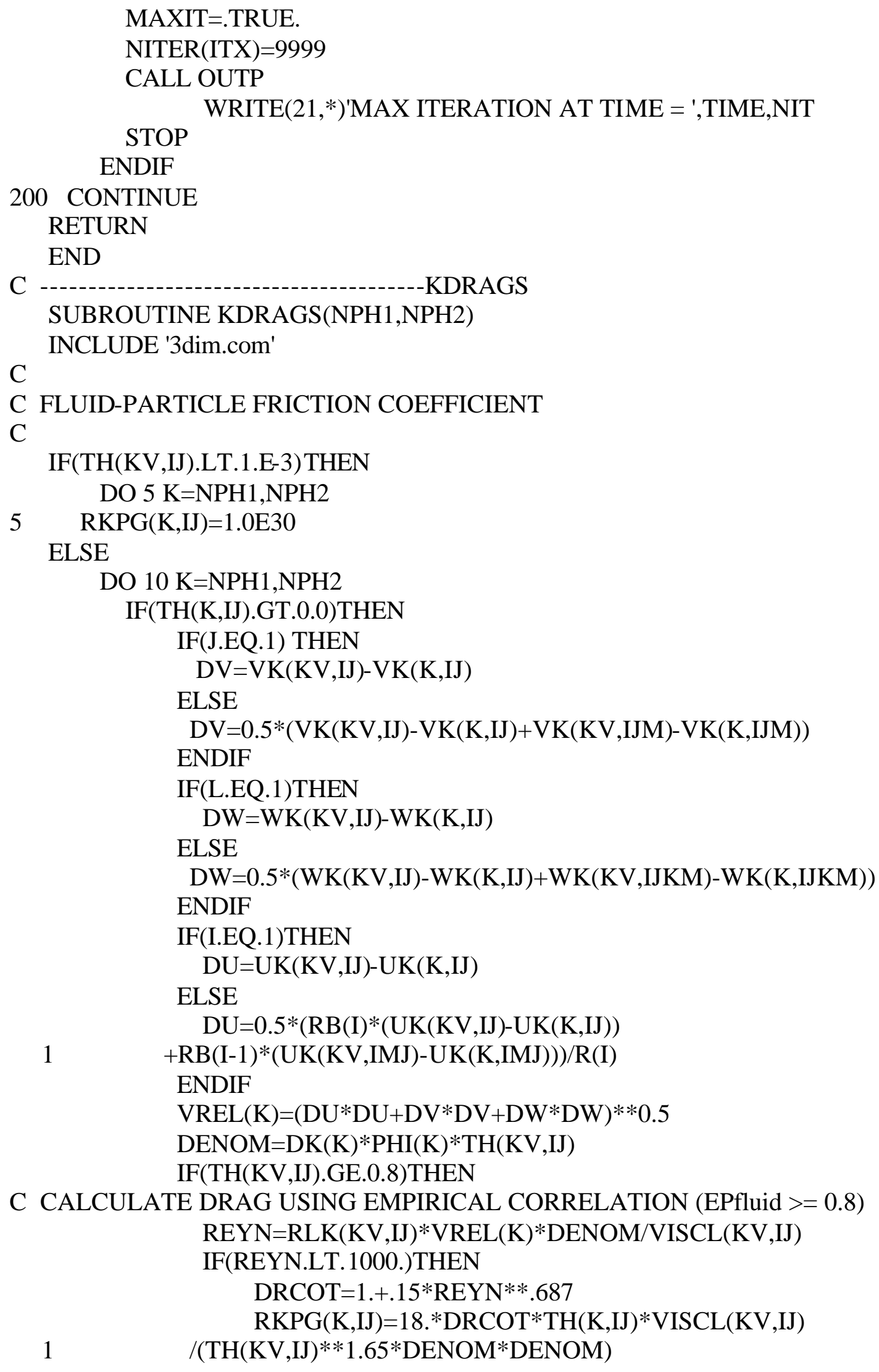


ELSE

$\mathrm{RKPG}(\mathrm{K}, \mathrm{IJ})=.75 * \mathrm{DRCOE} * \mathrm{TH}(\mathrm{K}, \mathrm{IJ}) * \operatorname{VREL}(\mathrm{K}) *$

1

$\mathrm{RLK}(\mathrm{KV}, \mathrm{IJ}) /(\mathrm{DENOM} * \mathrm{TH}(\mathrm{KV}, \mathrm{IJ}) * * 1.65)$

ENDIF

IF(RKPG(K,IJ).GT.1.0E30)RKPG(K,IJ)=1.0E30

ELSE

C CALCULATE DRAG USING ERGUN EQUATION (EPfluid < 0.8 )

$\operatorname{RKPG}(\mathrm{K}, \mathrm{IJ})=(150.0 *(1.0-\mathrm{TH}(\mathrm{KV}, \mathrm{IJ})) * \mathrm{VISCL}(\mathrm{KV}, \mathrm{IJ}) /$

1

$\mathrm{DENOM}+1.75 * \mathrm{RLK}(\mathrm{KV}, \mathrm{IJ}) * \mathrm{VREL}(\mathrm{K})) * \mathrm{TH}(\mathrm{K}, \mathrm{IJ}) / \mathrm{DENOM}$

ENDIF

IF(MODAB.NE.1)RKPG(K,IJ)=RKPG(K,IJ)/TH(KV,IJ)

$\mathrm{C}$

ELSE

$\operatorname{RKPG}(\mathrm{K}, \mathrm{IJ})=0.0$

ENDIF

10 CONTINUE

ENDIF

RETURN

END

C

SUBROUTINE MASFK(NPH1,NPH2)

$\mathrm{C}$

INCLUDE '3dim.com'

C CALCULATES MASS FLUXES FOR THE PHASES

$\mathrm{C}$

DO $10 \mathrm{~K}=\mathrm{NPH} 1, \mathrm{NPH} 2$

IF(UK(K,IMJ).GE.0.)THEN

$\operatorname{RLFRK}(\mathrm{K}, \mathrm{IMJ})=\mathrm{UK}(\mathrm{K}, \mathrm{IMJ}) * \mathrm{RLK}(\mathrm{K}, \mathrm{IJL}) * \mathrm{RB}(\mathrm{I}-1)$

ELSE

$\mathrm{RLFRK}(\mathrm{K}, \mathrm{IMJ})=\mathrm{UK}(\mathrm{K}, \mathrm{IMJ}) * \mathrm{RLK}(\mathrm{K}, \mathrm{IJ}) * \mathrm{RB}(\mathrm{I}-1)$

ENDIF

IF(VK(K,IJM).GE.0.)THEN

$\operatorname{RLFTK}(\mathrm{K}, \mathrm{IJM})=\mathrm{VK}(\mathrm{K}, \mathrm{IJM}) * \mathrm{RLK}(\mathrm{K}, \mathrm{IJB})$

ELSE

$\operatorname{RLFTK}(\mathrm{K}, \mathrm{IJM})=\mathrm{VK}(\mathrm{K}, \mathrm{IJM}) * \mathrm{RLK}(\mathrm{K}, \mathrm{IJ})$

ENDIF

IF(WK(K,IJKM).GE.0.)THEN

RLFAK $(\mathrm{K}, \mathrm{IJKM})=\mathrm{WK}(\mathrm{K}, \mathrm{IJKM}) * \mathrm{RLK}(\mathrm{K}, \mathrm{IJKF})$

ELSE

$\operatorname{RLFAK}(\mathrm{K}, \mathrm{IJKM})=\mathrm{WK}(\mathrm{K}, \mathrm{IJKM}) * \mathrm{RLK}(\mathrm{K}, \mathrm{IJ})$

ENDIF

10 CONTINUE

C

ENTRY MASFKA(NPH1,NPH2)

DO $20 \mathrm{~K}=\mathrm{NPH} 1, \mathrm{NPH} 2$

IF(UK(K,IJ).GE.0.)THEN 


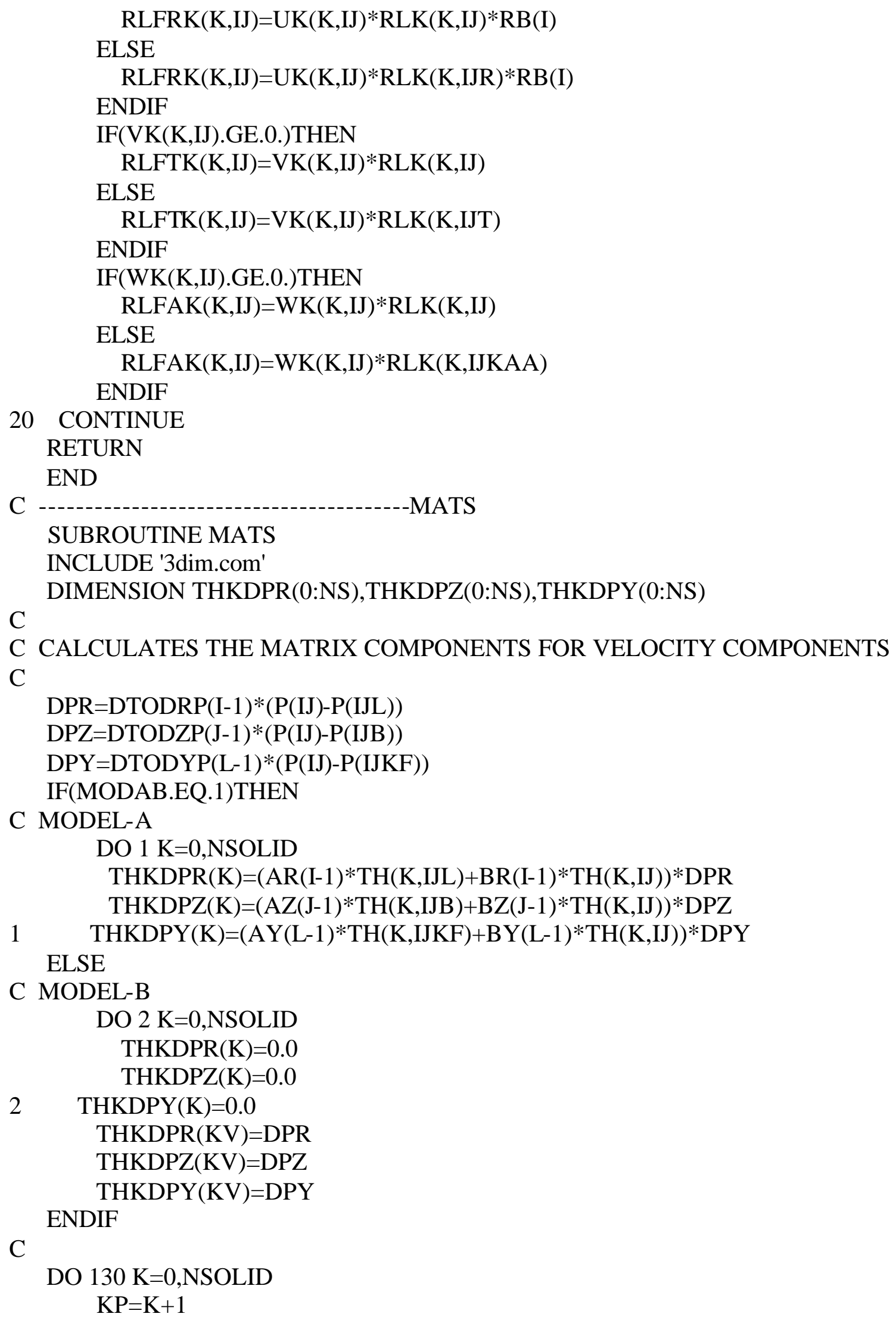




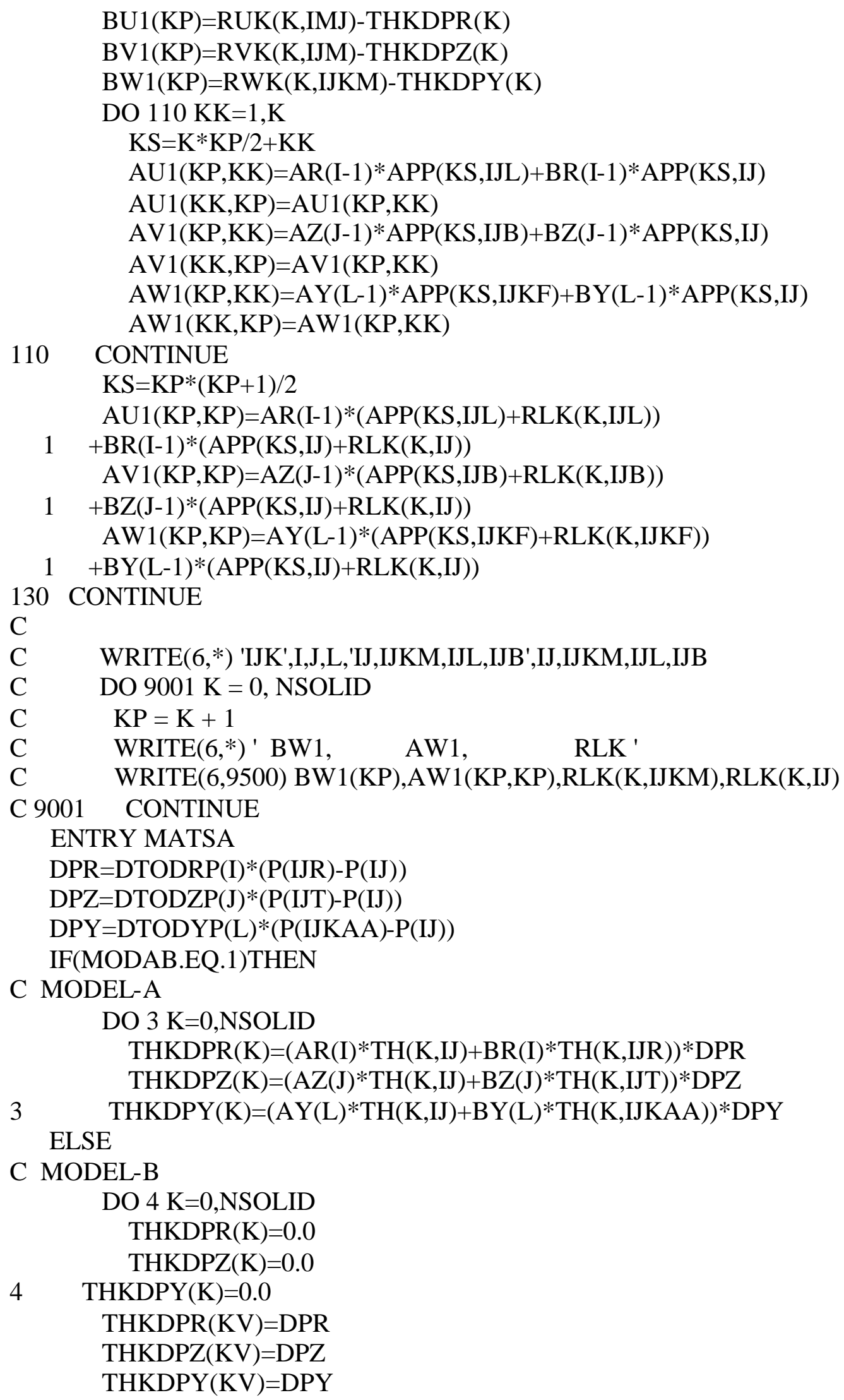




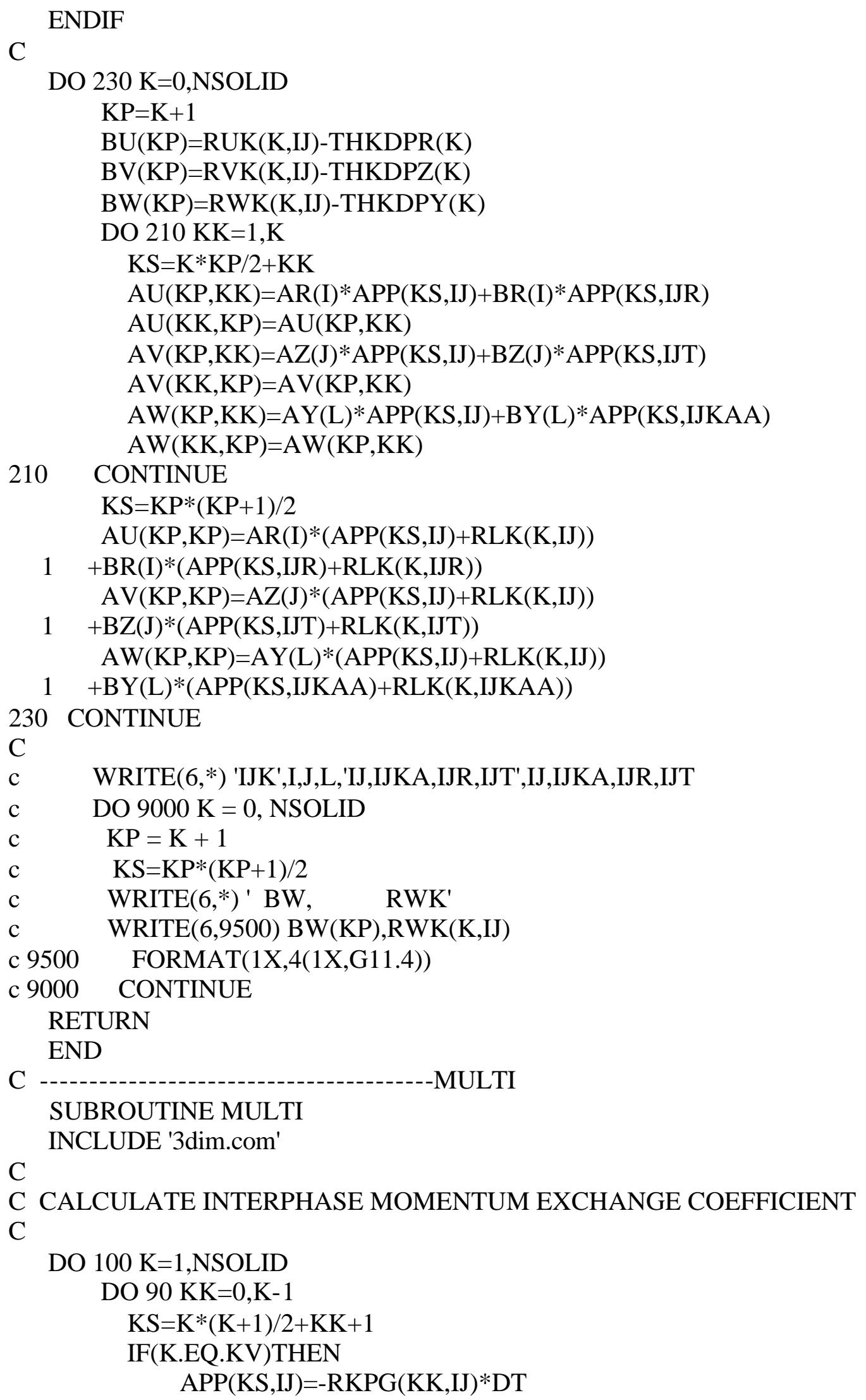




\section{ELSEIF(KK.EQ.KV)THEN}

ELSE

$$
\operatorname{APP}(K S, I J)=-R K P G(K, I J) * D T
$$

C CALCULATE PARTICLE TO PARTICLE INTERACTION

EPSUM $=\mathrm{TH}(\mathrm{K}, \mathrm{IJ})+\mathrm{TH}(\mathrm{KK}, \mathrm{IJ})$

IF(EPSUM.NE.0.0)THEN

IF(DK(K).GT.DK(KK))THEN

$\mathrm{K} 1=\mathrm{K}$

$\mathrm{K} 2=\mathrm{KK}$

ELSE

$\mathrm{K} 1=\mathrm{KK}$

$\mathrm{K} 2=\mathrm{K}$

ENDIF

$\mathrm{XBAR}=\mathrm{TH}(\mathrm{K} 1, \mathrm{IJ}) / \mathrm{EPSUM}$

IF(XBAR.LE.PHILIM(K,KK))THEN

$\mathrm{EPKL}=\mathrm{EPSL}(\mathrm{K}, \mathrm{KK}) * \mathrm{XBAR}+\mathrm{CPHI}(\mathrm{K} 2)$

ELSE

$\mathrm{EPKL}=\mathrm{EPSU}(\mathrm{K}, \mathrm{KK}) *(1.0-\mathrm{XBAR})+\mathrm{CPHI}(\mathrm{K} 1)$

ENDIF

$\mathrm{CEPR}=(\mathrm{EPSUM} / \mathrm{EPKL}) * *(1 . / 3$.

IF(CEPR.GE.1.0)THEN

$\mathrm{CON}=4 . \mathrm{E} 10 * \mathrm{RLK}(\mathrm{K}, \mathrm{IJ}) * \mathrm{RLK}(\mathrm{KK}, \mathrm{IJ}) * \mathrm{DT} * \mathrm{DKF}(\mathrm{K}, \mathrm{KK})$ ELSE

$\mathrm{CON}=\mathrm{DT} * \mathrm{RLK}(\mathrm{K}, \mathrm{IJ}) * \mathrm{RLK}(\mathrm{KK}, \mathrm{IJ}) * \mathrm{DKF}(\mathrm{K}, \mathrm{KK})$

1

$*(\mathrm{CEPR}+3) /.(1 .-\mathrm{CEPR})$

ENDIF

$\mathrm{IF}(\mathrm{MODAB} . \mathrm{NE} .0) \mathrm{CON}=\mathrm{CON} / \mathrm{TH}(\mathrm{KV}, \mathrm{IJ})$

IF(J.EQ.1) THEN

$\mathrm{DV}=\mathrm{VK}(\mathrm{KK}, \mathrm{IJ})-\mathrm{VK}(\mathrm{K}, \mathrm{IJ})$

ELSE

$\mathrm{DV}=0.5^{*}(\mathrm{VK}(\mathrm{KK}, \mathrm{IJ})-\mathrm{VK}(\mathrm{K}, \mathrm{IJ})+\mathrm{VK}(\mathrm{KK}, \mathrm{IJM})-$

1

$\mathrm{VK}(\mathrm{K}, \mathrm{IJM}))$

ENDIF

IF(L.EQ.1)THEN

$\mathrm{DW}=\mathrm{WK}(\mathrm{KK}, \mathrm{IJ})-\mathrm{WK}(\mathrm{K}, \mathrm{IJ})$

ELSE

$\mathrm{DW}=0.5^{*}(\mathrm{WK}(\mathrm{KK}, \mathrm{IJ})-\mathrm{WK}(\mathrm{K}, \mathrm{IJ})+\mathrm{WK}(\mathrm{KK}, \mathrm{IJKM})-$

1 $\mathrm{WK}(\mathrm{K}, \mathrm{IJKM}))$

ENDIF

IF(I.EQ.1)THEN

$\mathrm{DU}=\mathrm{UK}(\mathrm{KK}, \mathrm{IJ})-\mathrm{UK}(\mathrm{K}, \mathrm{IJ})$

ELSE

$\mathrm{DU}=0.5 *(\mathrm{RB}(\mathrm{I}) *(\mathrm{UK}(\mathrm{KK}, \mathrm{IJ})-\mathrm{UK}(\mathrm{K}, \mathrm{IJ}))$

1

$\left.+\mathrm{RB}(\mathrm{I}-1)^{*}(\mathrm{UK}(\mathrm{KK}, \mathrm{IMJ})-\mathrm{UK}(\mathrm{K}, \mathrm{IMJ}))\right) / \mathrm{R}(\mathrm{I})$

ENDIF

$\mathrm{VRELP}=(\mathrm{DU} * \mathrm{DU}+\mathrm{DV} * \mathrm{DV}+\mathrm{DW} * \mathrm{DW}) * * 0.5$ 


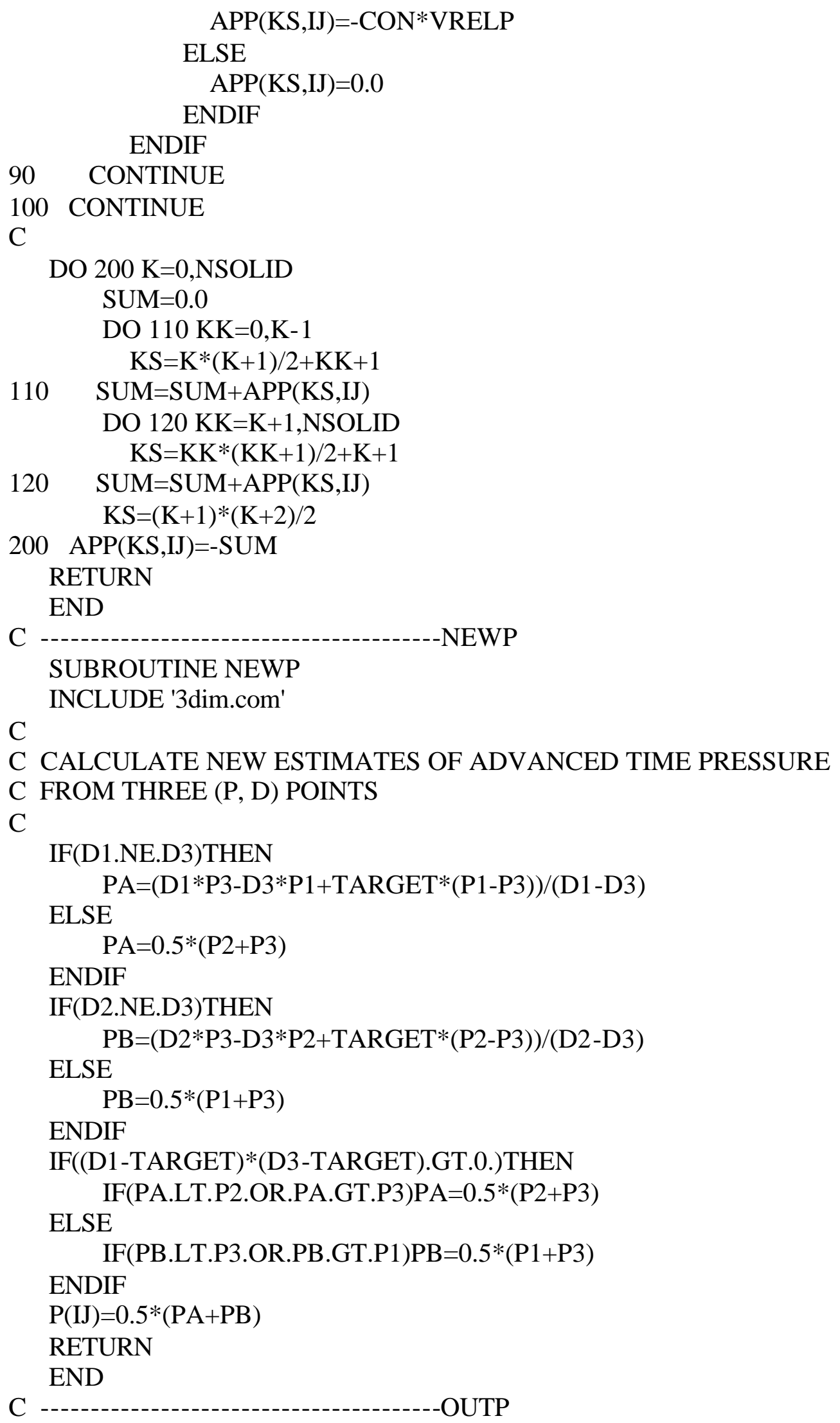


SUBROUTINE OUTP

INCLUDE '3dim.com'

CDTM

CDTM OUTPUT RESULTS TO DISK FOR FURTHER ANALYSIS

$\mathrm{C}$

DO $900 \mathrm{~L}=2, \mathrm{~KB} 1$

WRITE(22,*)' @@@K= ',L

WRITE(23,*)' @@@K=',L

WRITE(24,*) ' @@@ $@$ =',L

WRITE(25,*)' @@@K=',L

WRITE(26,*)' @@@K= ',L

WRITE(27,*)' @@@K=',L

WRITE(28,*)' @@@ $@$ = ',L

WRITE(29,*)' @@ @ = ',L

WRITE(30,*)' @@@K= ',L

if(NSOLID.eq.2)then

WRITE(31,*) '@@@K= ',L

WRITE(32,*) ' @@@K= ',L

WRITE(33,*)' @@@K= ',L

WRITE(34,*)' @@@ = ',L

endif

$\mathrm{I} 1=(\mathrm{L}) * \mathrm{IB} 2 * \mathrm{JB} 2+1$

WRITE $(22,548)$

DO $325 \mathrm{~J}=1$,JB2

$\mathrm{I} 1=\mathrm{I} 1-\mathrm{IB} 2$

$\mathrm{IJ} 2=\mathrm{IB} 2-1+\mathrm{I} 1$

325 WRITE(22,550) (P(IL), IL = I1,IJ2)

c $\quad$ DO $342 \mathrm{~K}=0$, NSOLID

$\mathrm{k}=0$

WRITE(23,549) K,K

$\mathrm{I} 1=(\mathrm{L}) * \mathrm{IB} 2 * \mathrm{JB} 2+1$

DO $228 \mathrm{~J}=1, \mathrm{JB} 2$

$\mathrm{I} 1=\mathrm{I} 1-\mathrm{IB} 2$

$\mathrm{IJ} 2=\mathrm{IB} 2-1+\mathrm{I} 1$

228 WRITE(23,550) (TH(K,IL), IL = I1,IJ2)

WRITE $(24,556) \mathrm{K}, \mathrm{K}$

$\mathrm{I} 1=(\mathrm{L}) * \mathrm{IB} 2 * \mathrm{JB} 2+1$

DO $236 \mathrm{~J}=1$,JB2

I1 = I1-IB2

$\mathrm{IJ} 2=\mathrm{IB} 2-1+\mathrm{I} 1$

236 WRITE(24,550) (VK(K,IL), IL = I1,IJ2)

WRITE $(25,557) \mathrm{K}, \mathrm{K}$

$\mathrm{I} 1=(\mathrm{L}) * \mathrm{IB} 2 * \mathrm{JB} 2+1$

DO $237 \mathrm{~J}=1, \mathrm{JB} 2$

I1 = I1-IB2 
$237 \quad$ IJ2 = IB2 - 1 + I1

WRITE(25,550) (UK(K,IL), IL = I1,IJ2)

WRITE(26,560) K,K

$\mathrm{I} 1=(\mathrm{L}) * \mathrm{IB} 2 * \mathrm{JB} 2+1$

DO $238 \mathrm{~J}=1$,JB2

$\mathrm{I} 1=\mathrm{I} 1-\mathrm{IB} 2$

$\mathrm{IJ} 2=\mathrm{IB} 2-1+\mathrm{I} 1$

238 WRITE $(26,550)(\mathrm{WK}(\mathrm{K}, \mathrm{IL}), \mathrm{IL}=\mathrm{I} 1, \mathrm{IJ} 2)$

$\mathrm{k}=1$

WRITE $(27,549) \mathrm{K}, \mathrm{K}$

$\mathrm{I} 1=(\mathrm{L}) * \mathrm{IB} 2 * \mathrm{JB} 2+1$

DO $328 \mathrm{~J}=1$,JB2

$\mathrm{I} 1=\mathrm{I} 1-\mathrm{IB} 2$

$\mathrm{IJ} 2=\mathrm{IB} 2-1+\mathrm{I} 1$

328 WRITE $(27,550)(\mathrm{TH}(\mathrm{K}, \mathrm{IL}), \mathrm{IL}=\mathrm{I} 1, \mathrm{IJ} 2)$

WRITE $(28,556) \mathrm{K}, \mathrm{K}$

$\mathrm{I} 1=(\mathrm{L}) * \mathrm{IB} 2 * \mathrm{JB} 2+1$

DO $336 \mathrm{~J}=1$,JB2

$\mathrm{I} 1=\mathrm{I} 1-\mathrm{IB} 2$

$\mathrm{IJ} 2=\mathrm{IB} 2-1+\mathrm{I} 1$

336 WRITE $(28,550)(\mathrm{VK}(\mathrm{K}, \mathrm{IL}), \mathrm{IL}=\mathrm{I} 1, \mathrm{IJ} 2)$

WRITE(29,557) K,K

$\mathrm{I} 1=(\mathrm{L}) * \mathrm{IB} 2 * \mathrm{JB} 2+1$

DO $337 \mathrm{~J}=1$,JB2

$\mathrm{I} 1=\mathrm{I} 1-\mathrm{IB} 2$

$\mathrm{IJ} 2=\mathrm{IB} 2-1+\mathrm{I} 1$

337 WRITE $(29,550)(\mathrm{UK}(\mathrm{K}, \mathrm{IL}), \mathrm{IL}=\mathrm{I} 1, \mathrm{IJ} 2)$

WRITE(30,560) K,K

$\mathrm{I} 1=(\mathrm{L}) * \mathrm{IB} 2 * \mathrm{JB} 2+1$

DO $338 \mathrm{~J}=1$,JB2

$\mathrm{I} 1=\mathrm{I} 1-\mathrm{IB} 2$

$\mathrm{IJ} 2=\mathrm{IB} 2-1+\mathrm{I} 1$

338 WRITE(30,550) (WK(K,IL), IL = I1,IJ2)

if(NSOLID.eq.2)then

$\mathrm{k}=2$

WRITE(31,549) K,K

$\mathrm{I} 1=(\mathrm{L}) * \mathrm{IB} 2 * \mathrm{JB} 2+1$

DO $428 \mathrm{~J}=1$,JB2

$\mathrm{I} 1=\mathrm{I} 1-\mathrm{IB} 2$

$\mathrm{IJ} 2=\mathrm{IB} 2-1+\mathrm{I} 1$

428 WRITE(31,550) (TH(K,IL), IL = I1,IJ2)

WRITE(32,556) K,K

$\mathrm{I} 1=(\mathrm{L}) * \mathrm{IB} 2 * \mathrm{JB} 2+1$

DO $436 \mathrm{~J}=1, \mathrm{JB} 2$ 


$$
\begin{aligned}
& \mathrm{I} 1=\mathrm{I} 1-\mathrm{IB} 2 \\
& \mathrm{IJ} 2=\mathrm{IB} 2-1+\mathrm{I} 1 \\
& 436 \text { WRITE(32,550) (VK(K,IL), IL = I1,IJ2) } \\
& \text { WRITE(33,557) K,K } \\
& \mathrm{I} 1=(\mathrm{L}) * \mathrm{IB} 2 * \mathrm{JB} 2+1 \\
& \text { DO } 437 \mathrm{~J}=1, \mathrm{JB} 2 \\
& \mathrm{I} 1=\mathrm{I} 1-\mathrm{IB} 2 \\
& \mathrm{IJ} 2=\mathrm{IB} 2-1+\mathrm{I} 1 \\
& \text { WRITE(34,560) K,K } \\
& \mathrm{I} 1=(\mathrm{L}) * \mathrm{IB} 2 * \mathrm{JB} 2+1 \\
& \text { DO } 438 \mathrm{~J}=1, \mathrm{JB} 2 \\
& \mathrm{I} 1=\mathrm{I} 1-\mathrm{IB} 2 \\
& \mathrm{IJ} 2=\mathrm{IB} 2-1+\mathrm{I} 1
\end{aligned}
$$

c342 CONTINUE

IF(INENT.NE.0) THEN

WRITE $(6,555) \mathrm{K}, \mathrm{K}$

$\mathrm{I} 1=(\mathrm{L}) * \mathrm{IB} 2 * \mathrm{JB} 2+1$

DO $339 \mathrm{~J}=1, \mathrm{JB} 2$

$\mathrm{I} 1=\mathrm{I} 1-\mathrm{IB} 2$

$\mathrm{IJ} 2=\mathrm{IB} 2-1+\mathrm{I} 1$

339 WRITE(6,550) (TL(K,IL), IL = I1,IJ2)

$$
\begin{aligned}
& \text { ENDIF } \\
& \text { IF(IKINT.EQ.1) THEN }
\end{aligned}
$$$$
\text { WRITE }(6,558) \mathrm{K}, \mathrm{K}
$$$$
\mathrm{I} 1=(\mathrm{L}) * \mathrm{IB} 2 * \mathrm{JB} 2+1
$$

$$
\begin{aligned}
& \text { DO } 340 \mathrm{~J}=1, \mathrm{JB} 2 \\
& \mathrm{I} 1=\mathrm{I} 1-\mathrm{IB} 2
\end{aligned}
$$$$
\mathrm{IJ} 2=\mathrm{IB} 2-1+\mathrm{I} 1
$$

340 WRITE(6,550) $(\mathrm{TSK}(\mathrm{K}, \mathrm{IL}), \mathrm{IL}=\mathrm{I} 1, \mathrm{IJ} 2)$

$$
\begin{aligned}
& \text { WRITE }(6,559) \mathrm{K}, \mathrm{K} \\
& \mathrm{I} 1=(\mathrm{L}) * \mathrm{IB} 2 * \mathrm{JB} 2+1
\end{aligned}
$$

DO $341 \mathrm{~J}=1, \mathrm{JB} 2$

$$
\begin{aligned}
& \mathrm{I} 1=\mathrm{I} 1-\mathrm{IB} 2 \\
& \mathrm{IJ} 2=\mathrm{IB} 2-1+\mathrm{I} 1
\end{aligned}
$$

341 WRITE(6,550) (VISCL(K,IL), IL = I1,IJ2)

$$
\text { ENDIF }
$$

c $\quad \mathrm{m}=\mathrm{m}+1$

900 CONTINUE

\section{RETURN}

547 FORMAT(1X,//,1X,'@ TIME = ',1PE12.5,' secs')

548 FORMAT(1X,/,1X,'FLUID PRESSURE, $\left.\mathrm{P}\left(\text { dynes } / \mathrm{cm}^{\wedge}{ }^{\wedge}\right)^{\prime} /\right)$

549 FORMAT(1X,/,1X,'VOLUME FRACTION (PHASE- ',I1 
1, ,'), TH',I1/)

550 FORMAT(1X,56(1X,G10.4))

555 FORMAT(1X,/,1X,'TEMPERATURE (PHASE-'

$\left.1, \mathrm{I} 1,{ }^{\prime}\right), \mathrm{TL}, \mathrm{I} 1,{ }^{\prime}($ Kelvin)'/)

556 FORMAT(1X,/,1X,'VELOCITY - Z (or Y) component,',

1 ' (PHASE-',I1,'), VK',I1,' (cm/s)'/)

557 FORMAT(1X,/,1X,'VELOCITY - R (or X) component,',

1 ' (PHASE-',I1,'), UK',I1,' (cm/s)'/)

558 FORMAT(1X,/,1X,'GRANULAR TEMPERATURE (PHASE-'

$\left.\left.1, \mathrm{I} 1,{ }^{\prime}\right), \mathrm{TSK}, \mathrm{I} 1,{ }^{\prime}\left((\mathrm{cm} / \mathrm{s})^{\wedge} 2\right)^{\prime} /\right)$

559 FORMAT(1X,/,1X,'GRANULAR SHEAR VISCOSITY (PHASE-'

1, I1,'), VISCL',I1,' (dynes/cm.s)'/)

560 FORMAT(1X,/,1X,'VELOCITY - THETA (or Z) component,',

1 ' (PHASE-',I1,'), WK',I1,' (cm/s)'/)

END

C

SUBROUTINE PROG

INCLUDE '3dim.com'

C

C CONTROL THE PROGRAM FLOW AND OUTPUT

$\mathrm{C}$

TDUMP1=TIME

TPRI=TIME

1 CONTINUE

TPDT $=$ TIME $+0.1 *$ DT

IF(MAXIT)CALL VARDT

$\mathrm{C}$

C SET BOUNDARY AND OBSTACLE CELLS

CALL BDRY

C

C SAVE VALUES AT PREVIOUS TIME STEP

DO $10 \mathrm{~L}=2, \mathrm{~KB} 2$

DO $10 \mathrm{~J}=2, \mathrm{JB} 2$

DO $10 \mathrm{I}=2$,IB2

$\mathrm{IJ}=\mathrm{I}+(\mathrm{J}-1) * \mathrm{IB} 2+(\mathrm{L}-1) * \mathrm{IB} 2 * \mathrm{JB} 2$

IF(IFL(IJ).NE.2.OR.IFL(IJ).EQ.3)THEN

IF(I.NE.IB2.OR.J.NE.JB2.OR.L.NE.KB2) THEN

$\mathrm{IMJ}=\mathrm{INDC}(\mathrm{IJ}, 2)$

$\mathrm{IJM}=\mathrm{INDC}(\mathrm{IJ}, 4)$

$\mathrm{IJKM}=\mathrm{INDC}(\mathrm{IJ}, 26)$

ELSE

$\mathrm{IMJ}=\mathrm{IJ}-1$

$\mathrm{IJM}=\mathrm{IJ}-\mathrm{IB} 2$

$\mathrm{IJKM}=\mathrm{IJ}-\mathrm{IB} 2 * \mathrm{JB} 2$

ENDIF

$\operatorname{RLX}(\mathrm{IJ})=\operatorname{RLK}(0, \mathrm{IJ})$ 


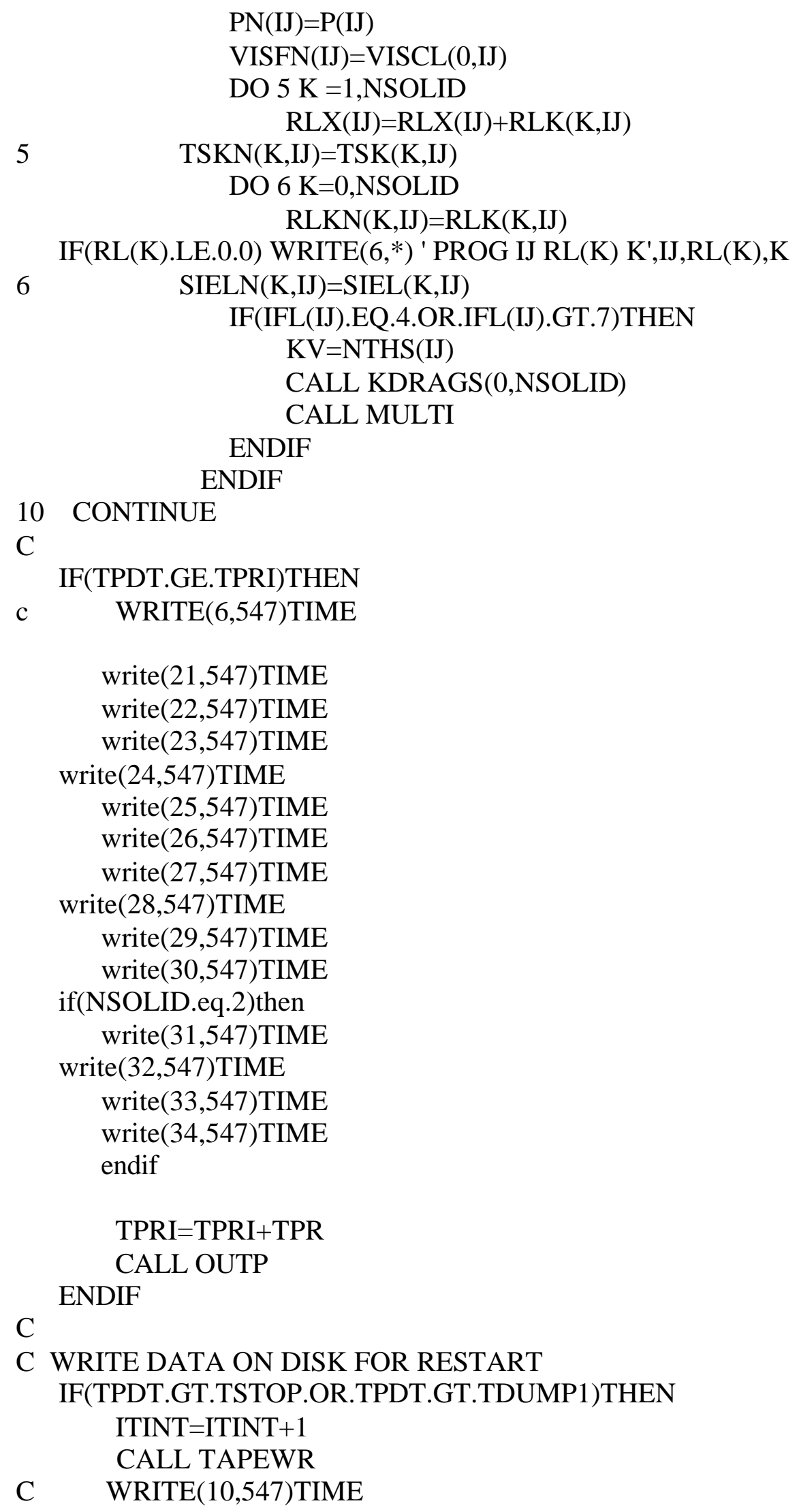




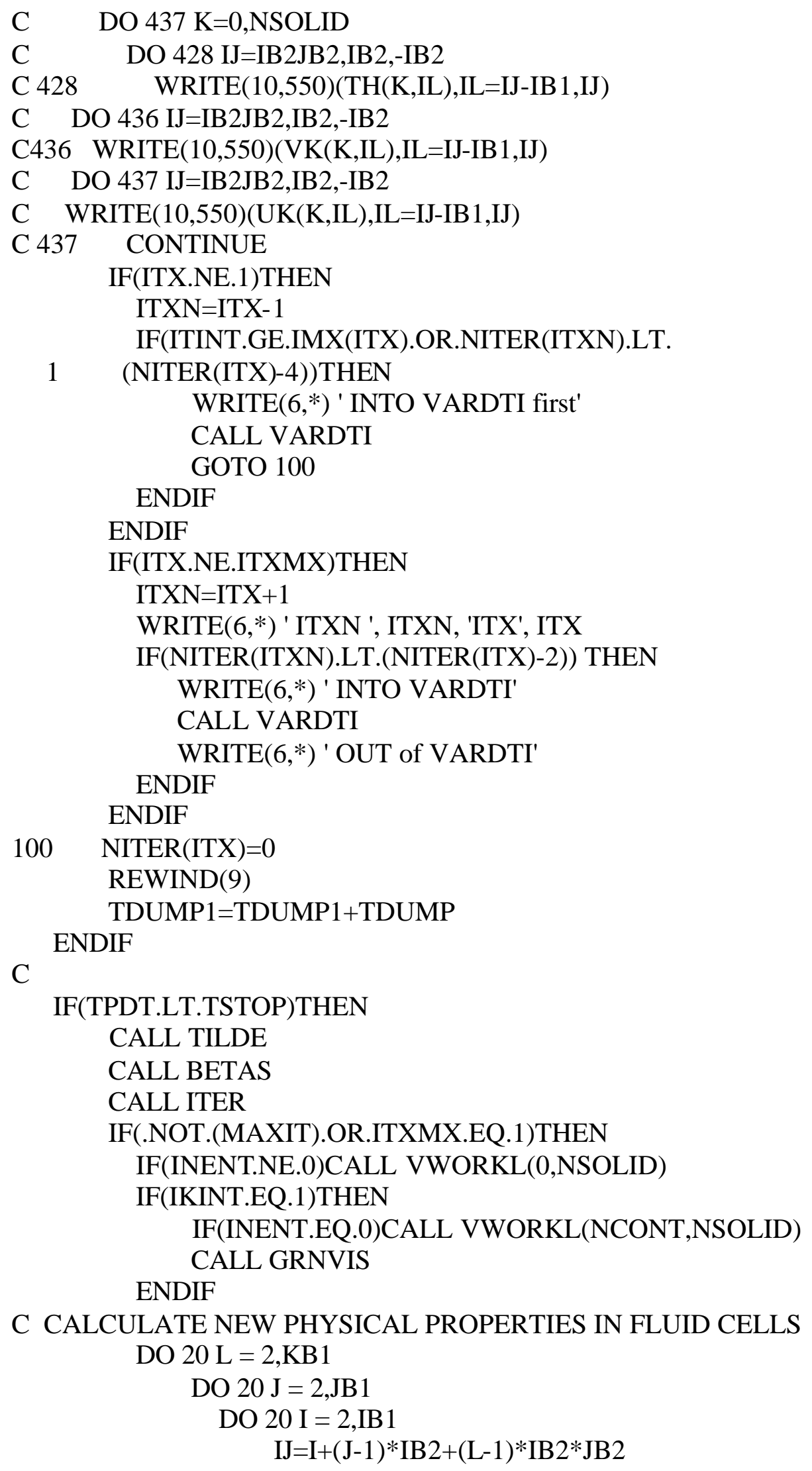




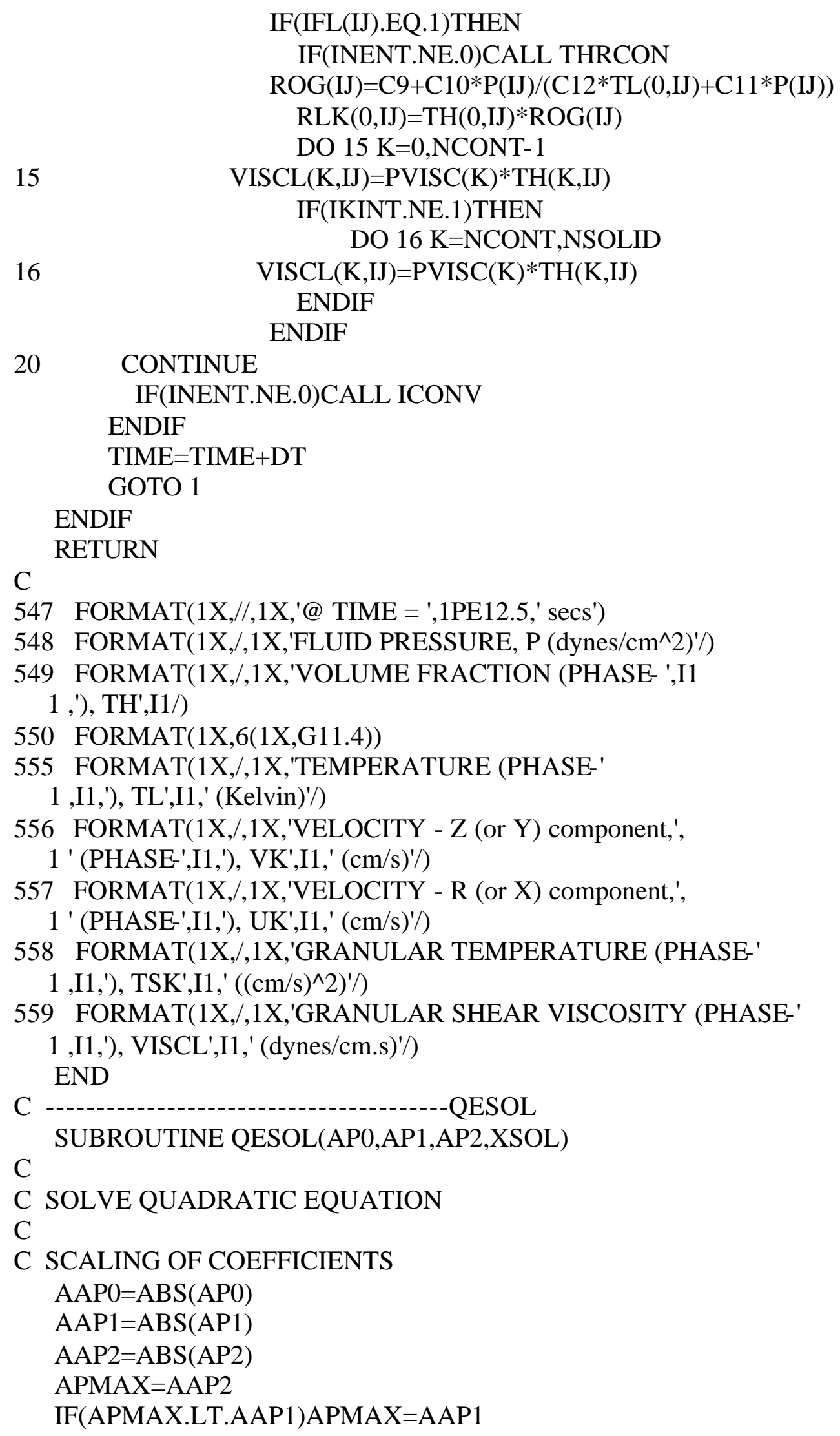




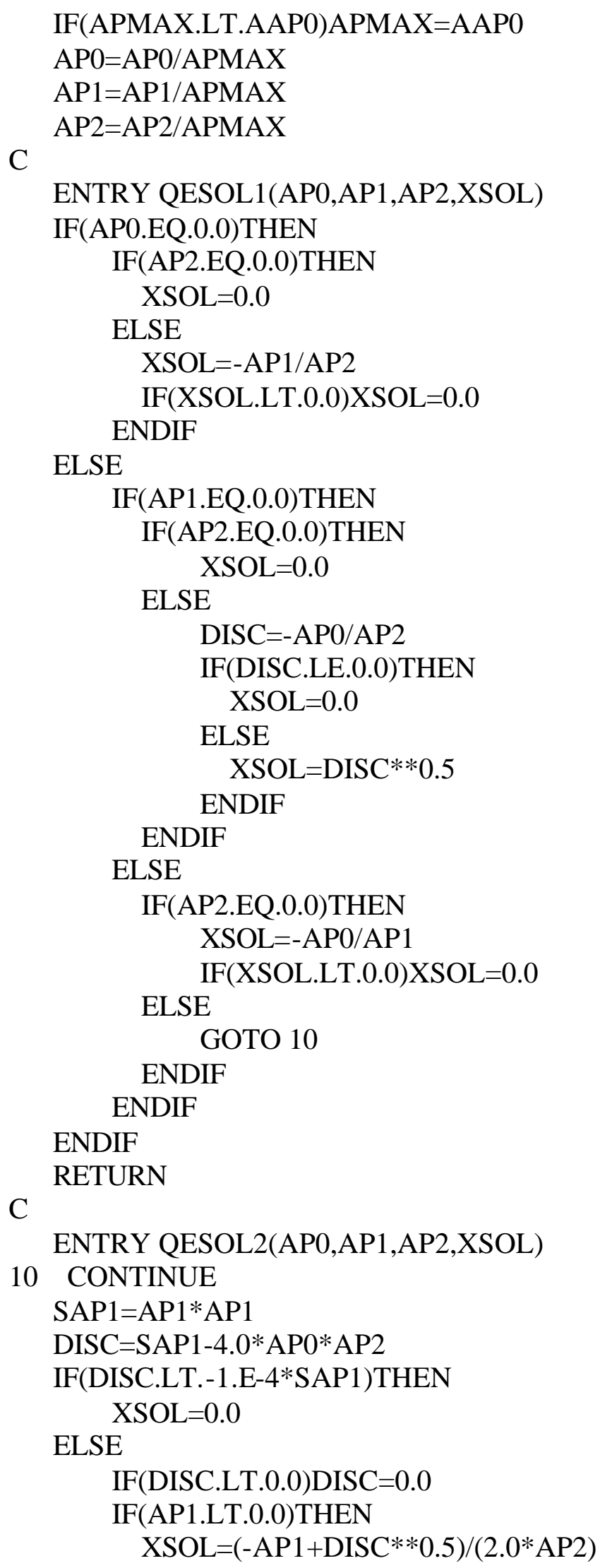


ELSE

$\mathrm{XSOL}=1 /(-2.0 * \mathrm{AP} 0 /(\mathrm{AP} 1+\mathrm{DISC} * * 0.5))$

ENDIF

ENDIF

RETURN

END

$\mathrm{C}$

SUBROUTINE RHEATS

$\mathrm{C}$

INCLUDE '3dim.com'

C INTERPASE HEAT TRANSFER COEFFICIENT

C RHEAT MUST BE GREATER THAN ZERO IF VOL. FRAC. $=0$ OR 1

$\mathrm{C}$

IF(KV.EQ.0)THEN

$\mathrm{AKGO}=8.67 \mathrm{E} 5 *(\mathrm{TL}(0, \mathrm{IJ}) / 1400.0) * * 1.786$

ELSE

$\mathrm{AKGO}=1.0$

ENDIF

$\mathrm{PR}=\mathrm{CL}(\mathrm{KV}) * \mathrm{VISCL}(\mathrm{KV}, \mathrm{IJ}) /(\mathrm{AKGO} * \mathrm{TH}(\mathrm{KV}, \mathrm{IJ}))$

$\mathrm{CRPR}=\mathrm{PR} * *(1 . / 3$.)

DO $10 \mathrm{~K}=1$,NSOLID

IF(RLK(K,IJ).GT.1.E-3*RLKMIN(K))THEN

$\mathrm{SP}=6 . * \mathrm{TH}(\mathrm{K}, \mathrm{IJ}) / \mathrm{DK}(\mathrm{K})$

REYN=TH(KV,IJ)*RLK(KV,IJ)*VREL $(\mathrm{K}) * \mathrm{DK}(\mathrm{K}) * \mathrm{PHI}(\mathrm{K})$

1 /VISCL(KV,IJ)

C GUNN'S MODEL 2/21/89

$\mathrm{PTH}=\mathrm{TH}(\mathrm{K}, \mathrm{IJ}) * \mathrm{TH}(\mathrm{K}, \mathrm{IJ})$

$\mathrm{PNU}=((2 .+5 . * \mathrm{PTH}) *(1 .+.7 * \mathrm{REYN} * * .2 * \mathrm{CRPR})$

$\left.1+(2 . / 15 .+1.2 * \mathrm{PTH}) * \mathrm{REYN}^{* *} .7 * \mathrm{CRPR}\right) * \mathrm{SP}$

$\operatorname{RHEAT}(\mathrm{K}, \mathrm{IJ})=\mathrm{PNU} * \mathrm{AKGO} / \mathrm{DK}(\mathrm{K})$

ELSE

$\operatorname{RHEAT}(\mathrm{K}, \mathrm{IJ})=0.0$

ENDIF

10 CONTINUE

RETURN

END

C

SUBROUTINE SETC

C

INCLUDE '3dim.com'

C INITIALIZES CONSTANTS AND FUNCTIONS

C

SQTPI=PI** 0.5

RSQTP=1./SQTPI

$\mathrm{C}$ INITIALIZE STATIC SOLIDS PRESSURE AND COHESIVE FORCE

DO $15 \mathrm{I}=0,1000$ 
THX=I/1000.

IF(ICOH.EQ.0)THEN

$\mathrm{COHF}(\mathrm{I})=0.0$

ELSE

$\mathrm{COHF}(\mathrm{I})=10 . * *(-\mathrm{C} 13 * \mathrm{THX}+\mathrm{C} 14)$

ENDIF

IF(IKINT.EQ.1.AND.(THX-THMIN).GT.1.E-10)THEN

C KINETIC THEORY OF GRANULAR SOLIDS REGIME $\mathrm{GTH}(\mathrm{I})=0.0$

ELSE

C SOLIDS STRESS (ELASTIC MODULUS G) REGIME $\mathrm{GTH}(\mathrm{I})=10 * * *(-\mathrm{C} 15 * \mathrm{THX}+\mathrm{C} 16)$

ENDIF

15 CONTINUE

$\mathrm{C}$

C CALCULATE VOLUME FRACTION FOR (RLIM particles/cm^3)

DO $20 \mathrm{~K}=0$,NSOLID

$\mathrm{VOLP}=\mathrm{PI} * \mathrm{DK}(\mathrm{K}) * \mathrm{DK}(\mathrm{K}) * \mathrm{DK}(\mathrm{K}) / 6.0$

$\operatorname{RLKMIN}(\mathrm{K})=\mathrm{RLIM} * \mathrm{RL}(\mathrm{K}) * \operatorname{VOLP}$

20 CONTINUE

C

DO $21 \mathrm{~K}=\mathrm{NCONT}$,NSOLID

$21 \quad \mathrm{DMFP}(\mathrm{K})=\mathrm{RL}(\mathrm{K}) * \mathrm{DK}(\mathrm{K}) /((2 . * * 0.5) * 6.0 * \mathrm{RADP})$

C

DO $50 \mathrm{~K}=\mathrm{NCONT,NSOLID}$

$\operatorname{VISDIL}(\mathrm{K})=5.0 * \mathrm{SQTPI} * \mathrm{RL}(\mathrm{K}) * \mathrm{DK}(\mathrm{K}) / 96.0$

C $\operatorname{GCDIL}(\mathrm{K})=(15.0 / 4.0) * \operatorname{VISDIL}(\mathrm{K})$

50 CONTINUE

$\mathrm{C}$

MAXIT=.FALSE

ITINT $=0$

DO 30 IX=1,ITXMX

$\operatorname{IMX}(\mathrm{IX})=105-5 * \mathrm{IX}$

NITER(IX) $=0$

30 CONTINUE

C

$\mathrm{DRCOE}=(24 . / 1000) *.(1 .+.15 * 1000 . * * .687)$

DO $55 \mathrm{~K}=1$,NSOLID

DO $55 \mathrm{KK}=0, \mathrm{~K}-1$

IF(DK(K).GT.DK(KK))THEN

$$
\mathrm{K} 1=\mathrm{K}
$$

$\mathrm{K} 2=\mathrm{KK}$

ELSE

$\mathrm{K} 1=\mathrm{KK}$

ENDIF

$$
\mathrm{K} 2=\mathrm{K}
$$




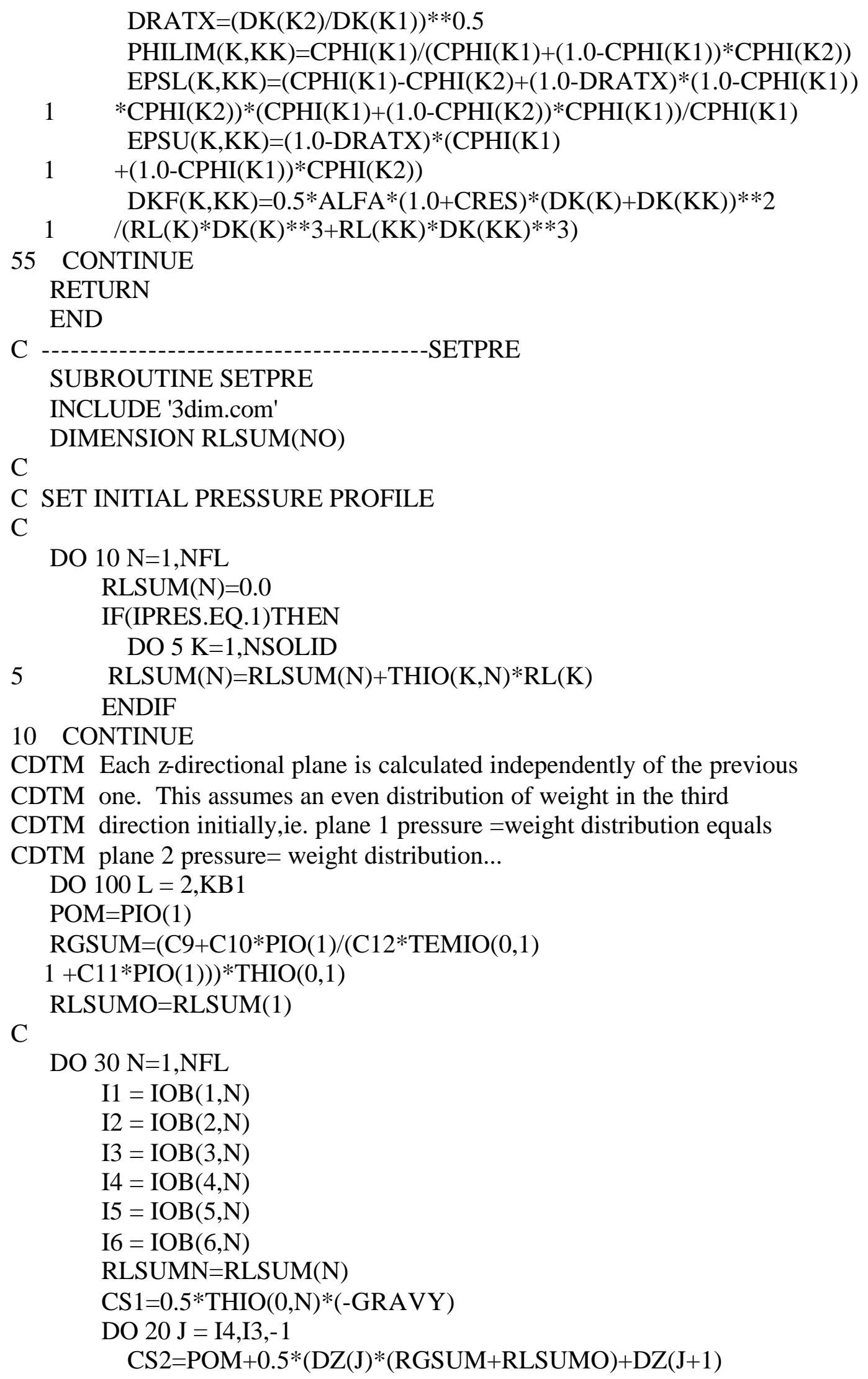




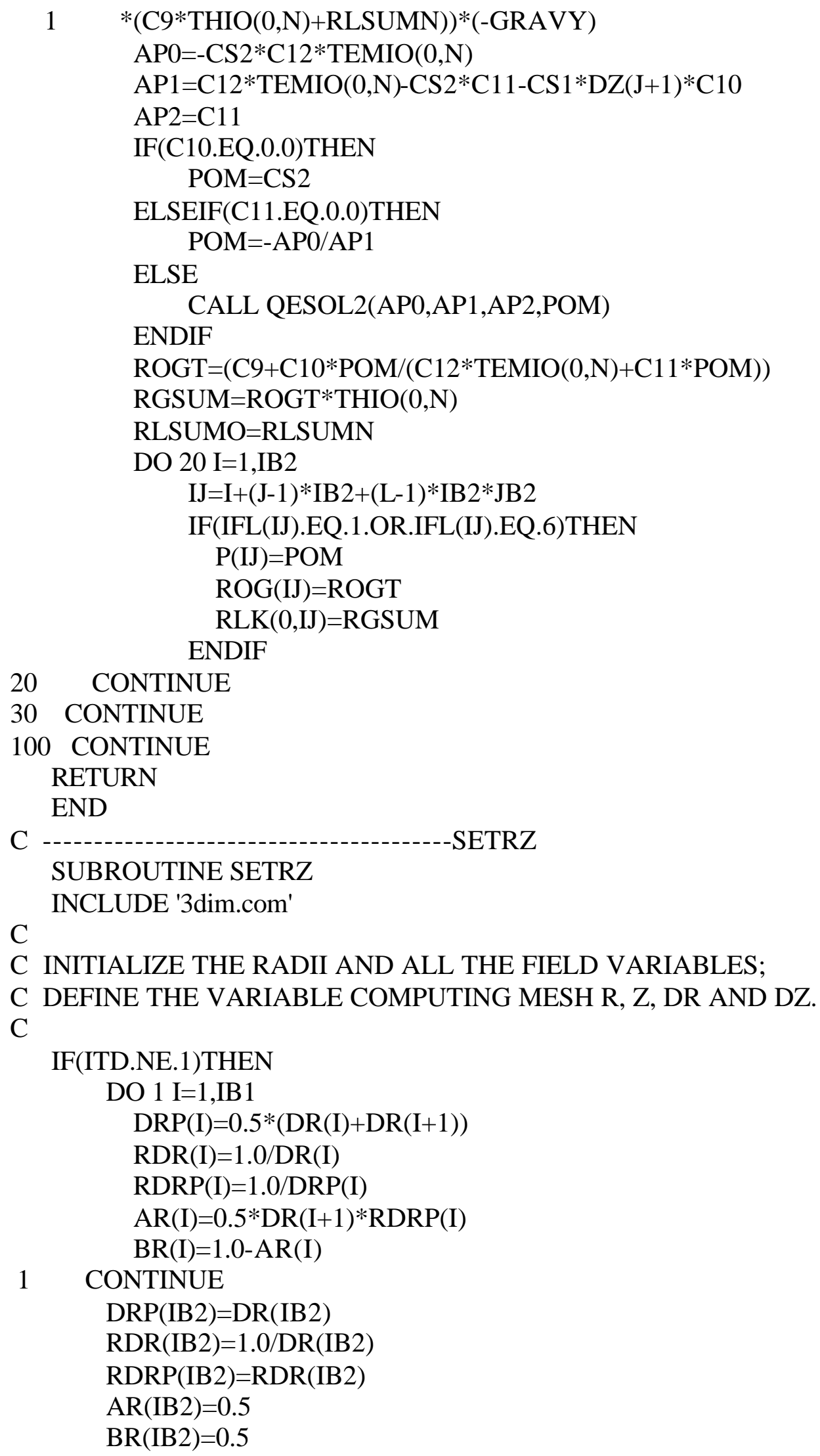




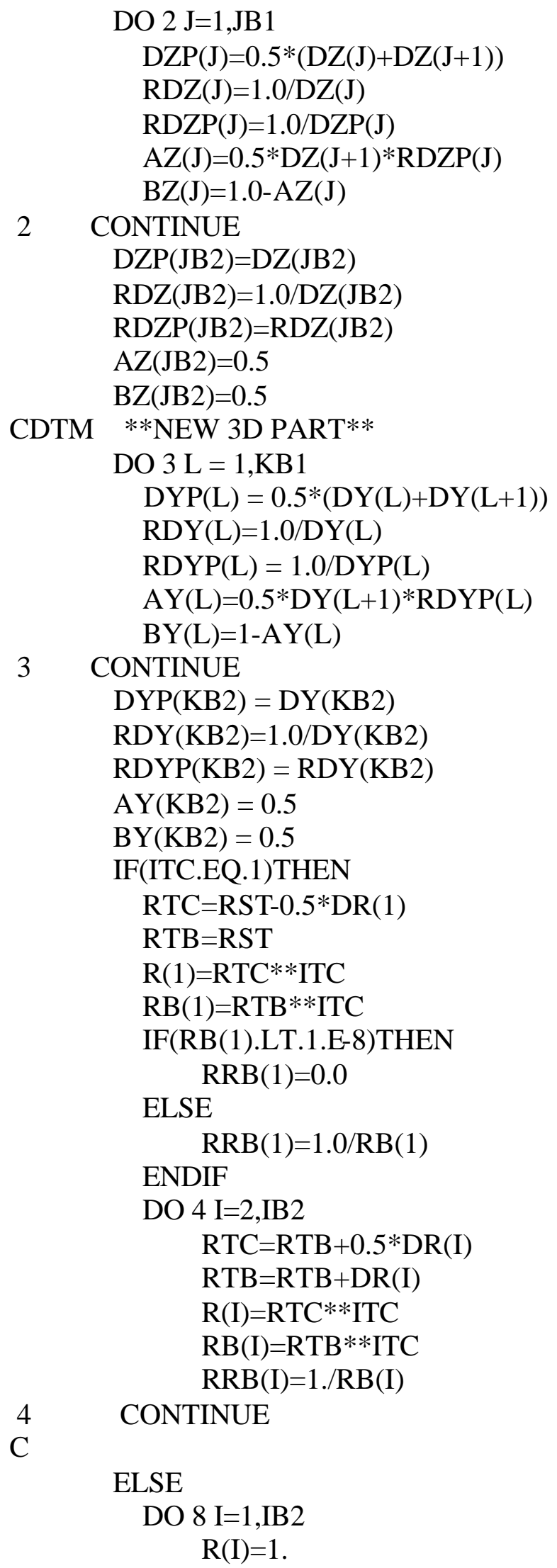




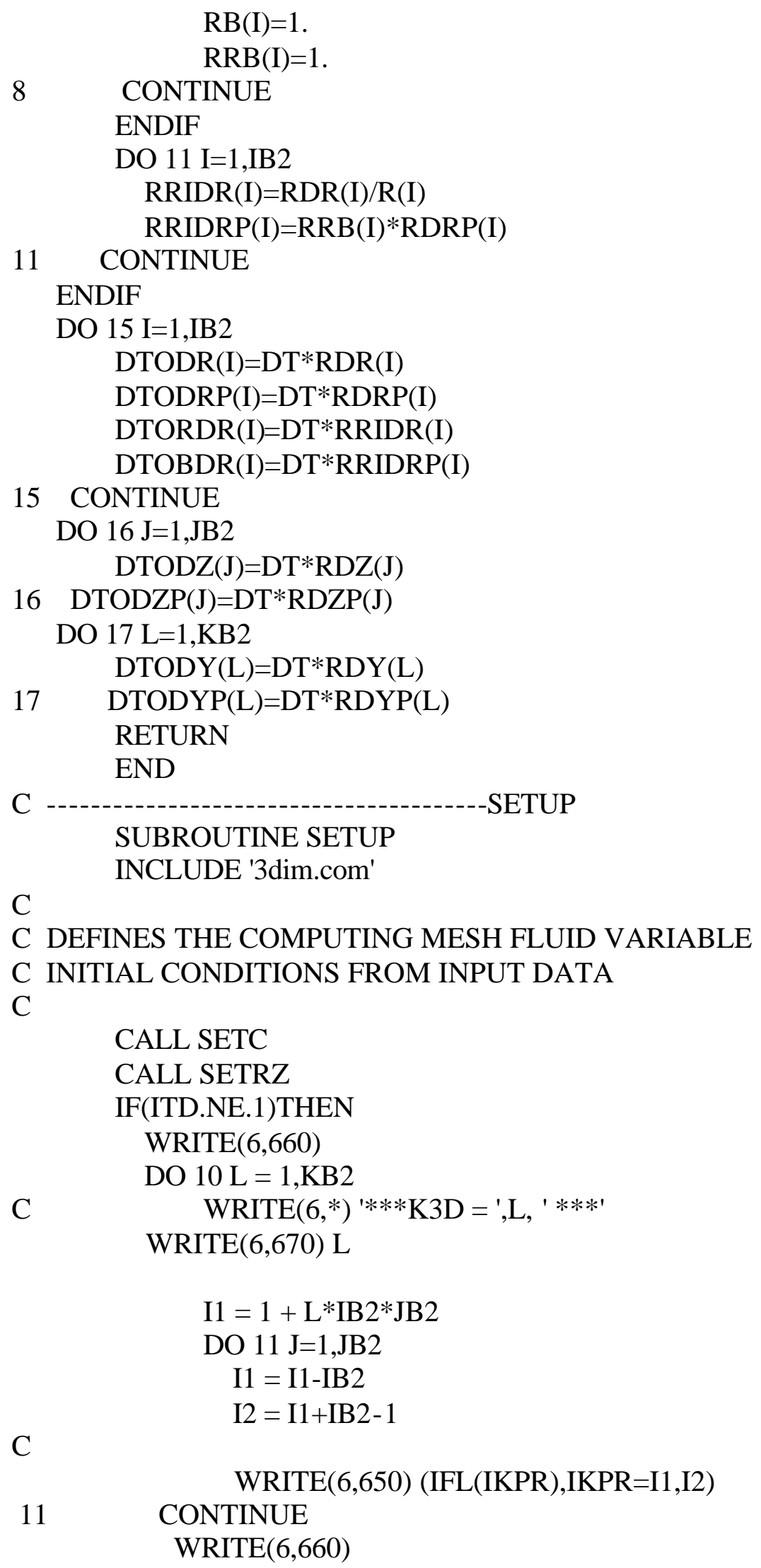


$\mathrm{C}$

C CALCULATE INDICES

CALL INDX

C

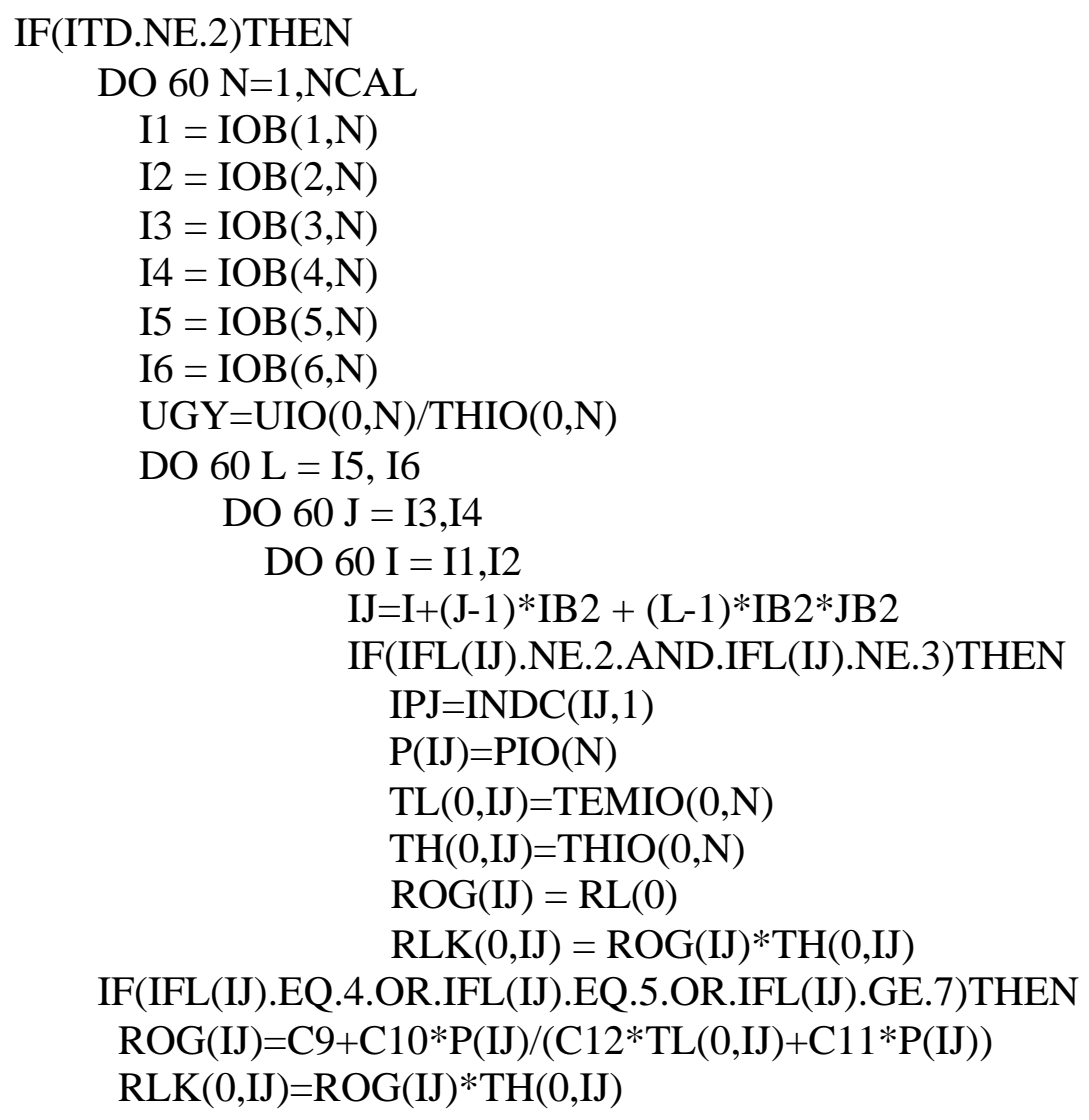

IF(IFL(IJ).EQ.4.OR.IFL(IJ).EQ.5.OR.IFL(IJ).GE.7)THEN

$\mathrm{ROG}(\mathrm{IJ})=\mathrm{C} 9+\mathrm{C} 10 * \mathrm{P}(\mathrm{IJ}) /(\mathrm{C} 12 * \mathrm{TL}(0, \mathrm{IJ})+\mathrm{C} 11 * \mathrm{P}(\mathrm{IJ}))$

$\operatorname{RLK}(0, \mathrm{IJ})=\mathrm{ROG}(\mathrm{IJ}) * \mathrm{TH}(0, \mathrm{IJ})$

CDTM

\section{ENDIF}

$$
\mathrm{UK}(0, \mathrm{IJ})=\mathrm{UIO}(0, \mathrm{~N})
$$

$\begin{array}{ll}\text { c } & \\ \text { c } & 1\end{array}$

IF(IFLZB.EQ.1.AND.IFL(IJ).NE.5.AND.

IFL(IPJ).NE.2.AND.IFL(IPJ).NE.3)UK(0,IJ)=UGY $\mathrm{VK}(0, \mathrm{IJ})=\mathrm{VIO}(0, \mathrm{~N})$

$\mathrm{WK}(0, \mathrm{IJ})=\mathrm{WIO}(0, \mathrm{~N})$

DO $56 \mathrm{~K}=1$,NSOLID

$\mathrm{TL}(\mathrm{K}, \mathrm{IJ})=\mathrm{TEMIO}(\mathrm{K}, \mathrm{N})$

$\mathrm{UK}(\mathrm{K}, \mathrm{IJ})=\mathrm{UIO}(\mathrm{K}, \mathrm{N})$

$\mathrm{VK}(\mathrm{K}, \mathrm{IJ})=\mathrm{VIO}(\mathrm{K}, \mathrm{N})$

$\mathrm{WK}(\mathrm{K}, \mathrm{IJ})=\mathrm{WIO}(\mathrm{K}, \mathrm{N})$

$\operatorname{TSK}(\mathrm{K}, \mathrm{IJ})=\operatorname{TSKIO}(\mathrm{K}, \mathrm{N})$

$\mathrm{TH}(\mathrm{K}, \mathrm{IJ})=\mathrm{THIO}(\mathrm{K}, \mathrm{N})$

$\operatorname{RLK}(\mathrm{K}, \mathrm{IJ})=\mathrm{RL}(\mathrm{K}) * \mathrm{THIO}(\mathrm{K}, \mathrm{N})$ 


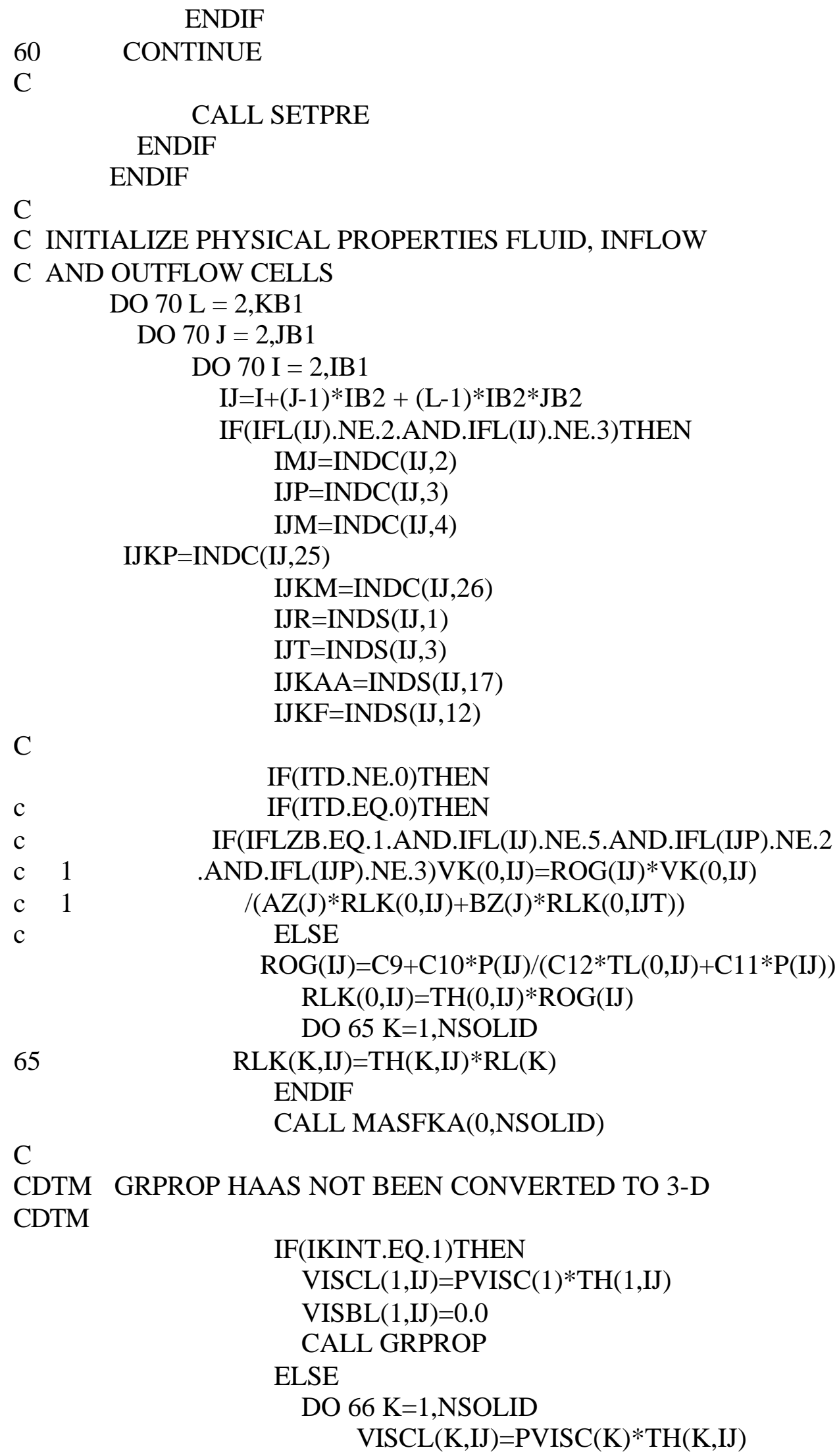




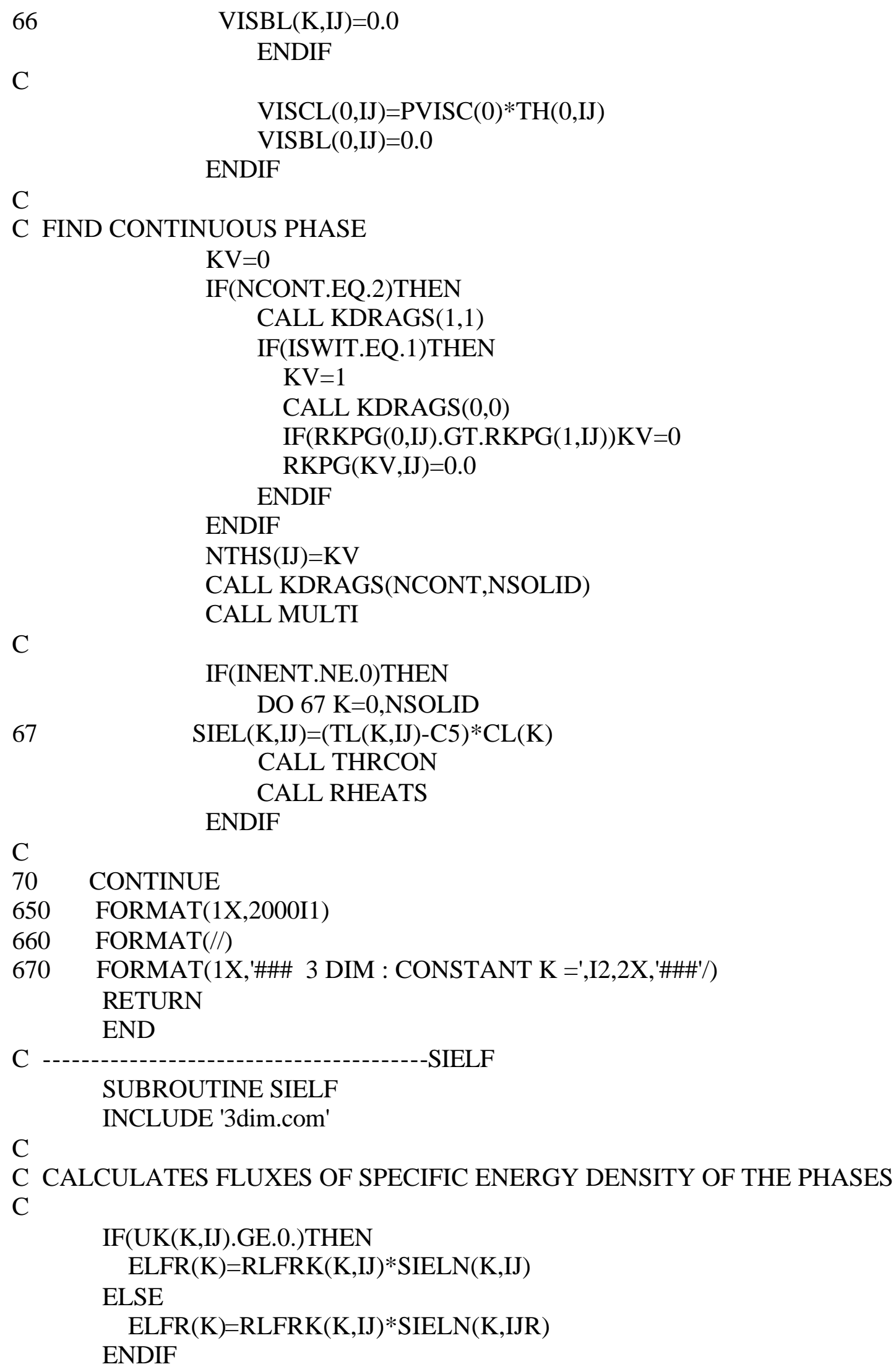




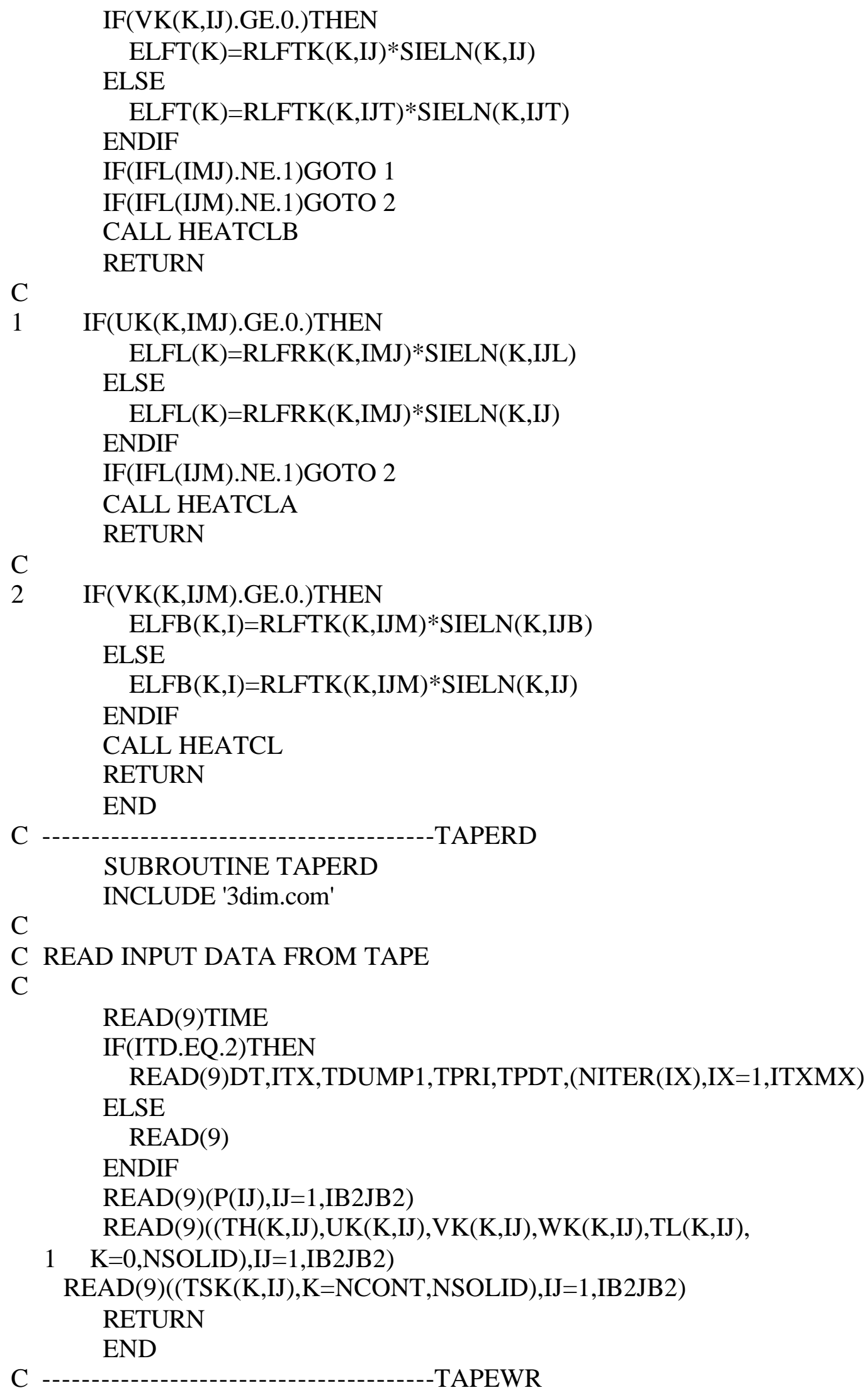


SUBROUTINE TAPEWR

INCLUDE '3dim.com'

C

C WRITE INPUT DATA TO TAPE

C

WRITE(9)TIME,IB2,JB2,KB2,NSOLID

WRITE(9)DT,ITX,TDUMP1,TPRI,TPDT,(NITER(IX),IX=1,ITXMX)

WRITE(9)(P(IJ),IJ=1,IB2JB2)

WRITE(9)((TH(K,IJ),UK(K,IJ),VK(K,IJ),WK(K,IJ),TL(K,IJ),

$1 \mathrm{~K}=0$,NSOLID),IJ=1,IB2JB2)

WRITE(9)((TSK(K,IJ),K=NCONT,NSOLID),IJ=1,IB2JB2)

RETURN

END

$\mathrm{C}$

SUBROUTINE THRCON

INCLUDE '3dim.com'

$\mathrm{C}$

PARAMETER (PIC=7.26E-3,RKP=0.3289,APIC=1.-PIC)

C DAMKOHLER'S EQUIVALENT THERMAL CONDUCTIVITY

$\mathrm{C}$

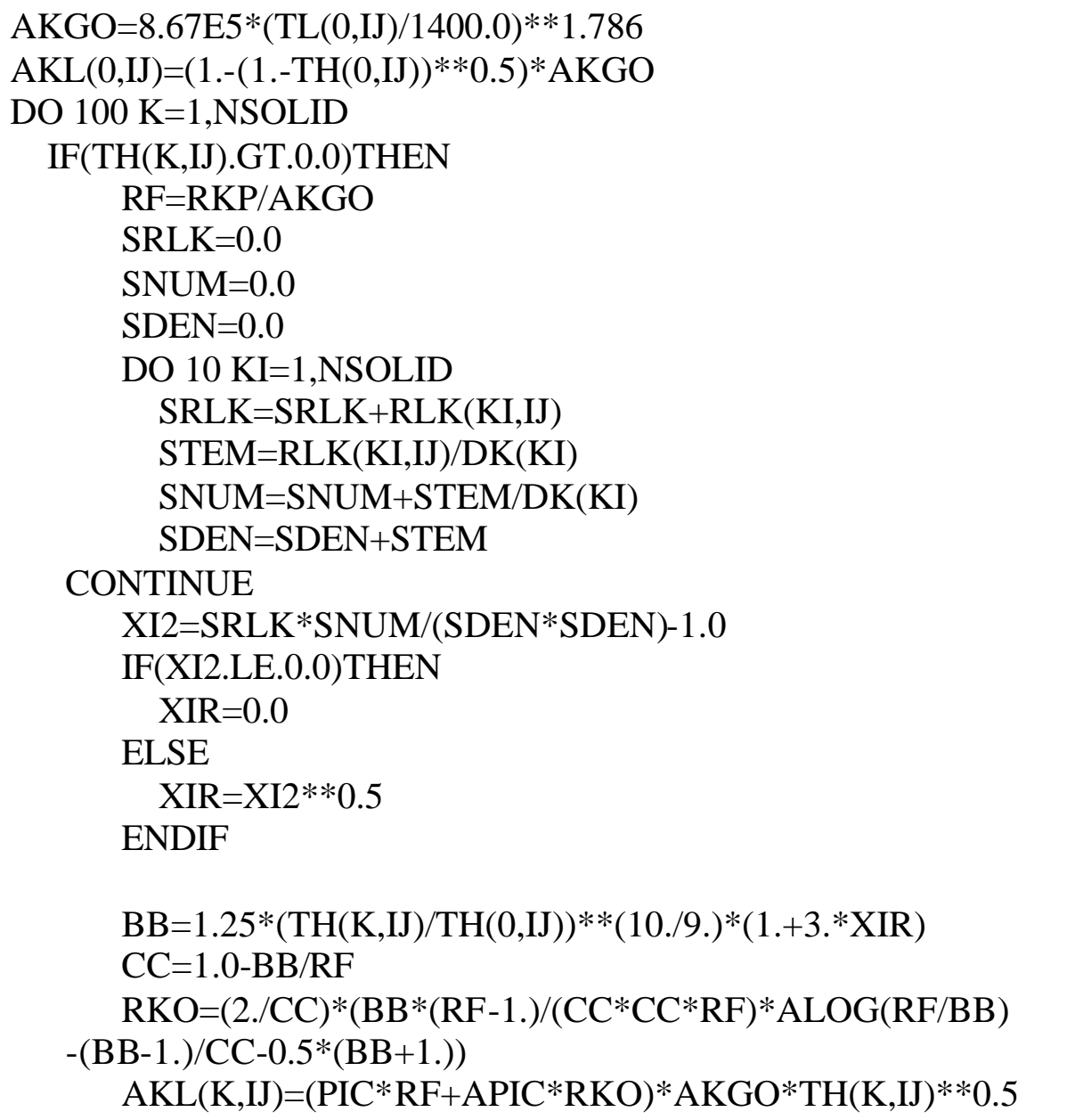




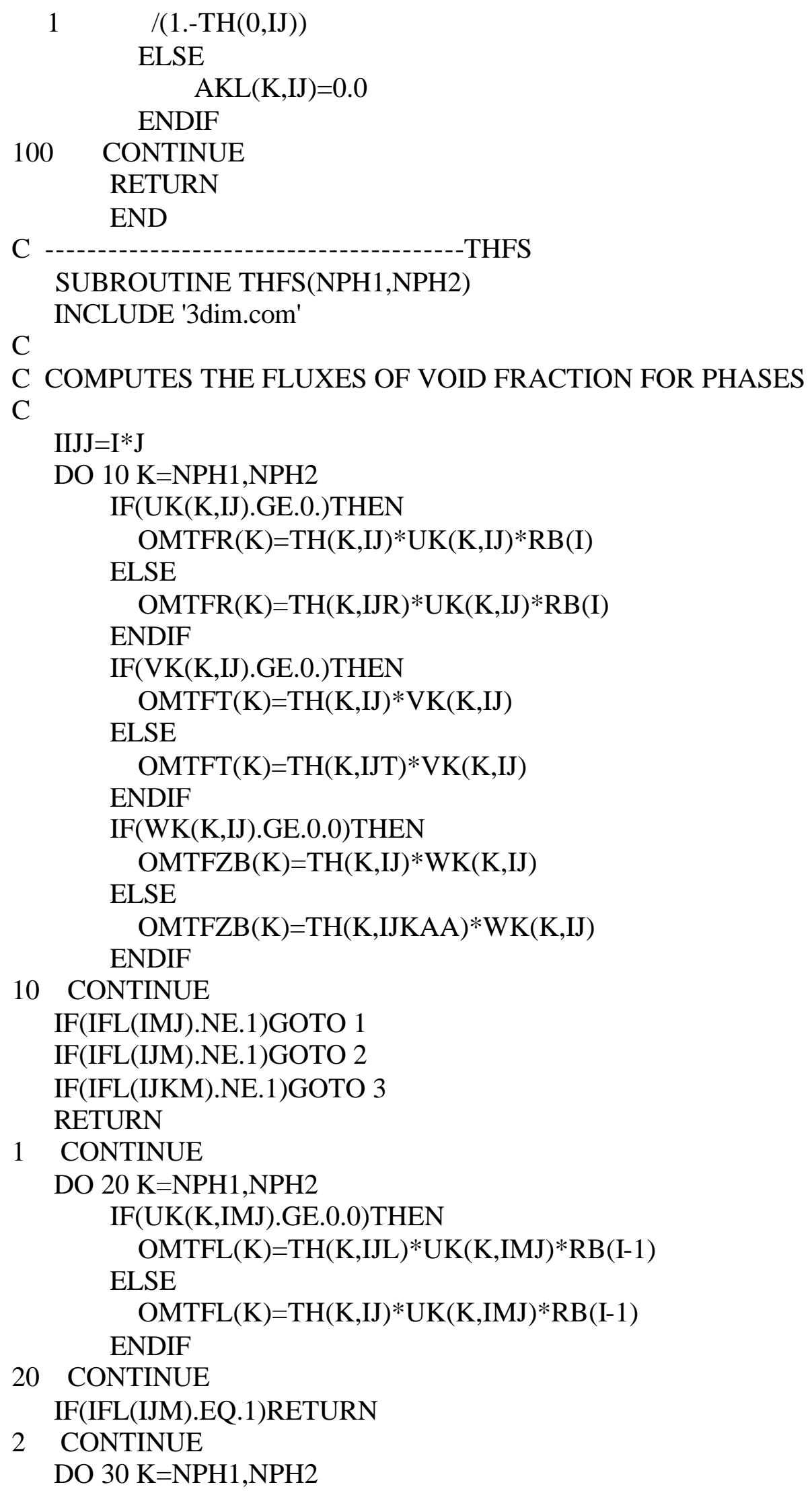


IF(VK(K,IJM).GE.0.0)THEN

$\operatorname{OMTFB}(\mathrm{K}, \mathrm{I})=\mathrm{TH}(\mathrm{K}, \mathrm{IJB}) * \mathrm{VK}(\mathrm{K}, \mathrm{IJM})$

ELSE

$\operatorname{OMTFB}(\mathrm{K}, \mathrm{I})=\mathrm{TH}(\mathrm{K}, \mathrm{IJ}) * \mathrm{VK}(\mathrm{K}, \mathrm{IJM})$

ENDIF

30 CONTINUE

IF(IFL(IJKM).EQ.1)RETURN

3 CONTINUE

DO $40 \mathrm{~K}=\mathrm{NPH} 1, \mathrm{NPH} 2$

IF(WK(K,IJKM).GE.0.0)THEN

OMTFZF $(\mathrm{K}, \mathrm{IIJJ})=\mathrm{TH}(\mathrm{K}, \mathrm{IJKF}) * \mathrm{WK}(\mathrm{K}, \mathrm{IJKM})$

ELSE

$\operatorname{OMTFZF}(\mathrm{K}, \mathrm{IIJJ})=\mathrm{TH}(\mathrm{K}, \mathrm{IJ}) * \mathrm{WK}(\mathrm{K}, \mathrm{IJKM})$

ENDIF

40 CONTINUE

RETURN

END

$\mathrm{C}$

TILDE

SUBROUTINE TILDE

INCLUDE '3dim.com'

DIMENSION RKR(0:NS),RKZ(0:NS),RKRAB(0:NS),RKZAB(0:NS)

DIMENSION RLGR(0:NS),RLGZ(0:NS)

DIMENSION RKY(0:NS),RKYAB(0:NS),RLGY(0:NS)

$\mathrm{C}$

C CALCULATE MOMENTA DUE TO CONVECTION, GRAVITY,

C VISCOUS STRESS, SOLIDS PRESSURE AND COHESIVE STRESS

C

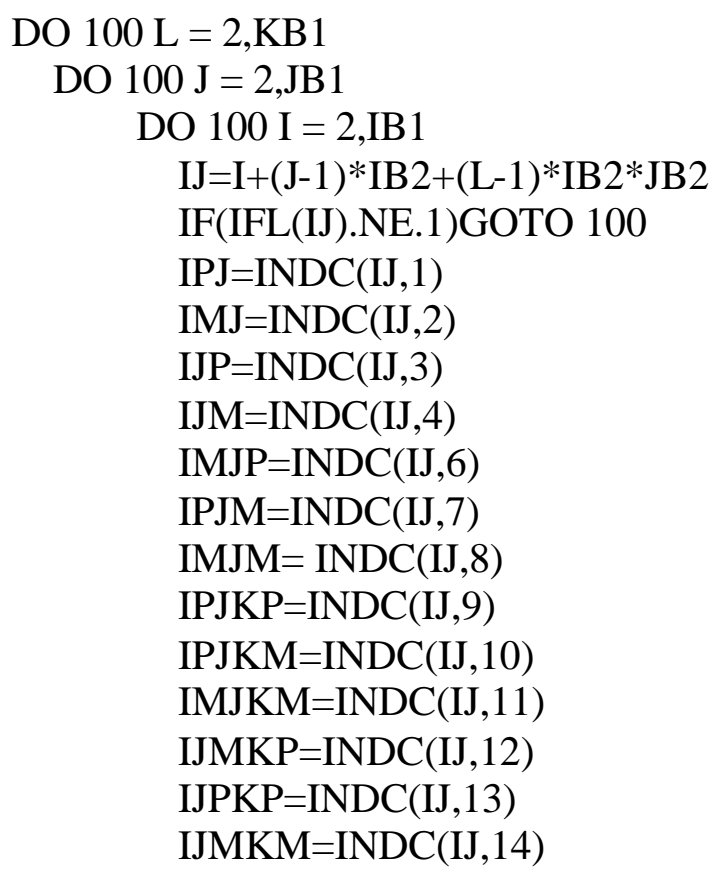




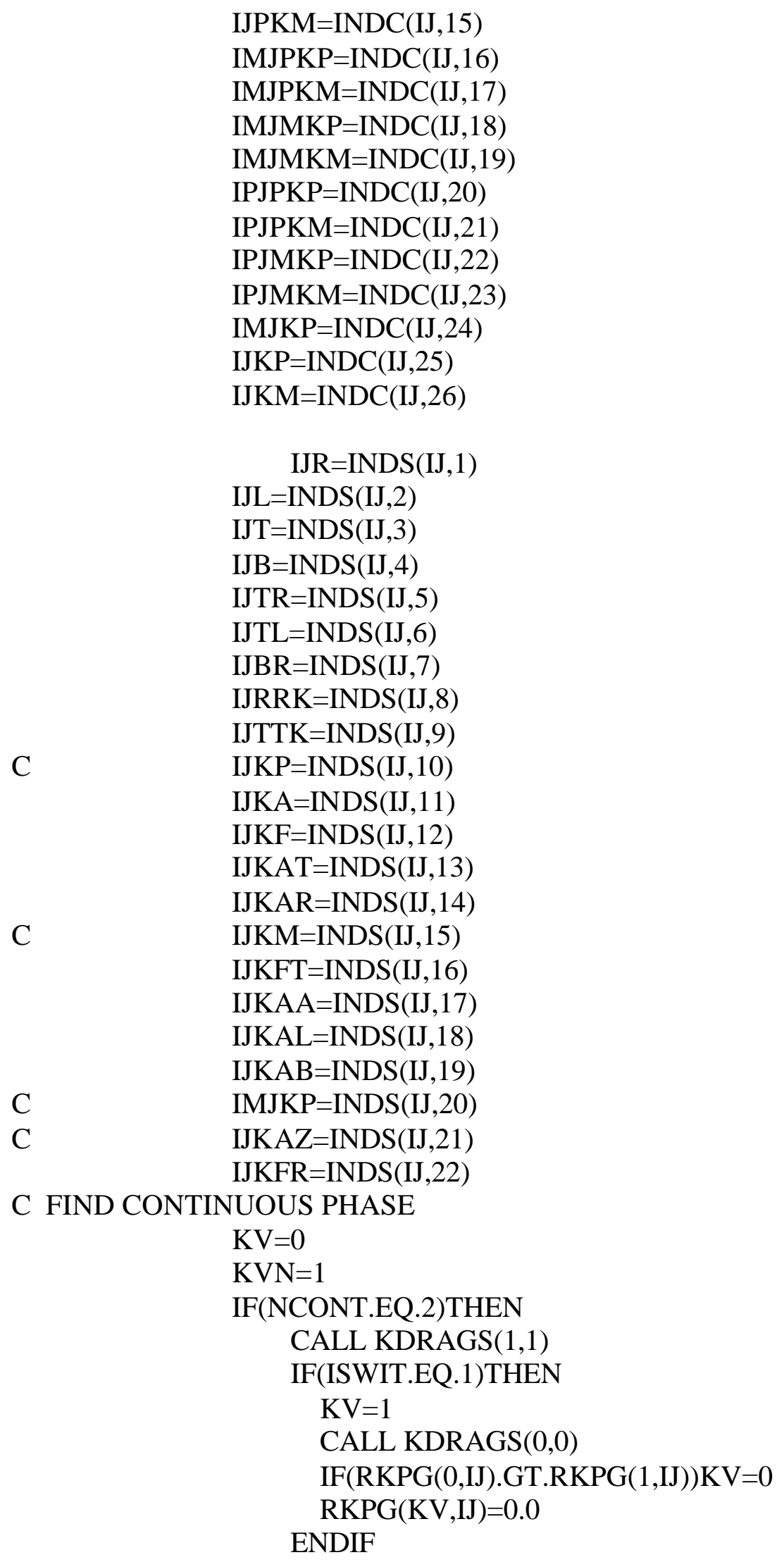




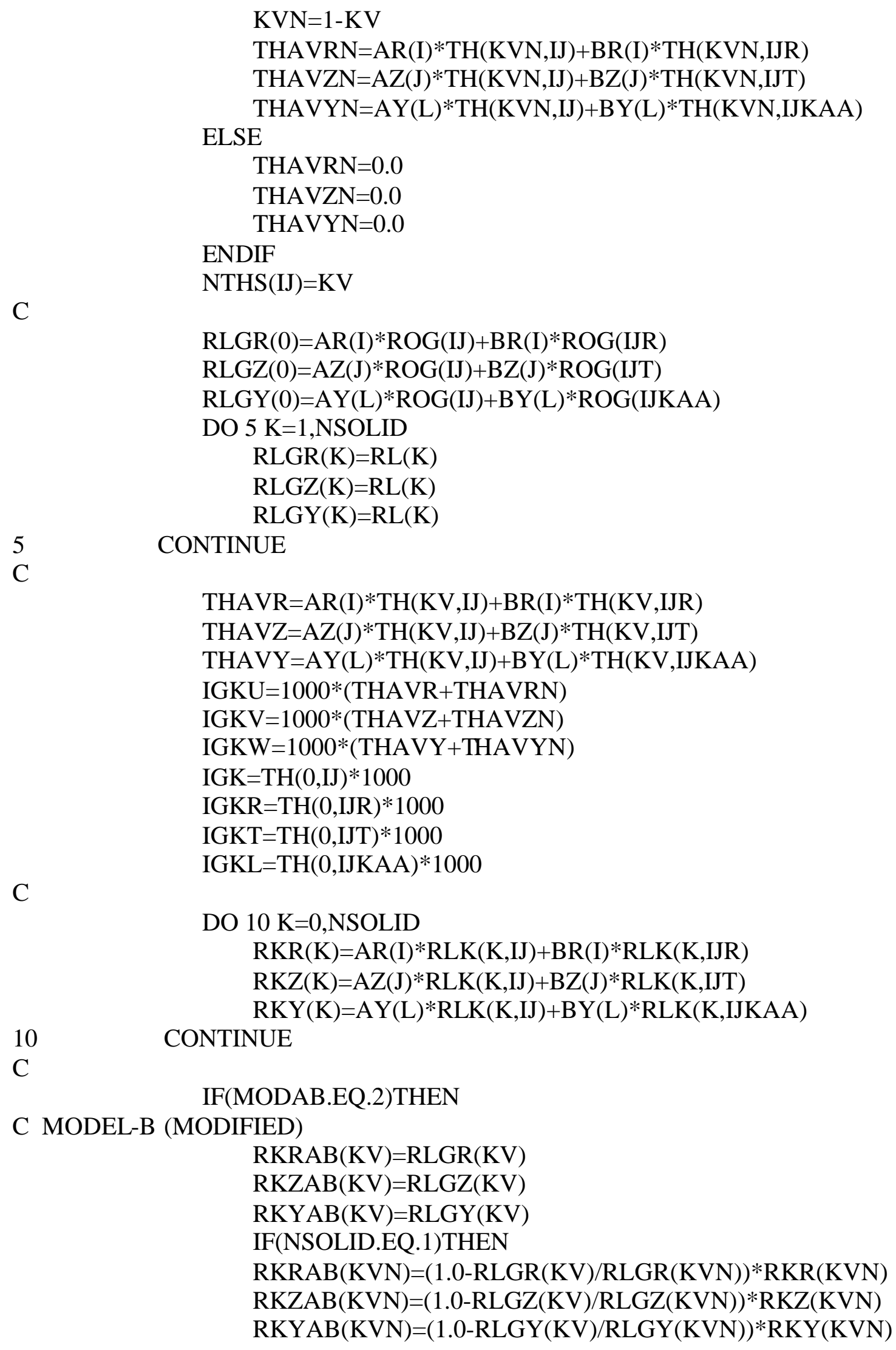

10

CONTINUE

DO $10 \mathrm{~K}=0$,NSOLID

$\mathrm{RKR}(\mathrm{K})=\mathrm{AR}(\mathrm{I}) * \mathrm{RLK}(\mathrm{K}, \mathrm{IJ})+\mathrm{BR}(\mathrm{I}) * \mathrm{RLK}(\mathrm{K}, \mathrm{IJR})$

$\mathrm{RKZ}(\mathrm{K})=\mathrm{AZ}(\mathrm{J}) * \mathrm{RLK}(\mathrm{K}, \mathrm{IJ})+\mathrm{BZ}(\mathrm{J}) * \mathrm{RLK}(\mathrm{K}, \mathrm{IJT})$ $\mathrm{RKY}(\mathrm{K})=\mathrm{AY}(\mathrm{L}) * \mathrm{RLK}(\mathrm{K}, \mathrm{IJ})+\mathrm{BY}(\mathrm{L}) * \mathrm{RLK}(\mathrm{K}, \mathrm{IJKAA})$

C

IF(MODAB.EQ.2)THEN

C MODEL-B (MODIFIED)

$\operatorname{RKRAB}(K V)=R L G R(K V)$

$\mathrm{RKZAB}(\mathrm{KV})=\mathrm{RLGZ}(\mathrm{KV})$

$\operatorname{RKYAB}(\mathrm{KV})=\mathrm{RLGY}(\mathrm{KV})$

IF(NSOLID.EQ.1)THEN

$\operatorname{RKRAB}(\mathrm{KVN})=(1.0-\mathrm{RLGR}(\mathrm{KV}) / \mathrm{RLGR}(\mathrm{KVN})) * \mathrm{RKR}(\mathrm{KVN})$

$\operatorname{RKZAB}(\mathrm{KVN})=(1.0-\mathrm{RLGZ}(\mathrm{KV}) / \mathrm{RLGZ}(\mathrm{KVN})) * \operatorname{RKZ}(\mathrm{KVN})$

$\operatorname{RKYAB}(\mathrm{KVN})=(1.0-\mathrm{RLGY}(\mathrm{KV}) / \mathrm{RLGY}(\mathrm{KVN})) * \operatorname{RKY}(\mathrm{KVN})$ 
ELSE

1

1

1

1

1

20

C MODEL-A

30

C

\author{
DO $20 \mathrm{~K}=0$,NSOLID \\ IF(K.NE.KV)THEN \\ $\operatorname{RKRAB}(\mathrm{K})=\operatorname{RKR}(\mathrm{K}) *(1.0-(\mathrm{AR}(\mathrm{I}) *$
}

$\mathrm{RLX}(\mathrm{IJ})+\mathrm{BR}(\mathrm{I}) * \mathrm{RLX}(\mathrm{IJR}))$

/RLGR(K))/THAVR $\mathrm{RKZAB}(\mathrm{K})=\mathrm{RKZ}(\mathrm{K}) *(1.0-(\mathrm{AZ}(\mathrm{J}) *$

$\mathrm{RLX}(\mathrm{IJ})+\mathrm{BZ}(\mathrm{J}) * \mathrm{RLX}(\mathrm{IJT}))$

/RLGZ(K))/THAVZ $\operatorname{RKYAB}(\mathrm{K})=\mathrm{RKY}(\mathrm{K}) *(1.0-(\mathrm{AY}(\mathrm{L}) *$

$\mathrm{RLX}(\mathrm{IJ})+\mathrm{BY}(\mathrm{L}) * \mathrm{RLX}(\mathrm{IJKAA}))$

/RLGY(K))/THAVY

ENDIF

CONTINUE

ENDIF

ELSE

DO $30 \mathrm{~K}=0$,NSOLID

$\operatorname{RKRAB}(\mathrm{K})=\operatorname{RKR}(\mathrm{K})$

$\operatorname{RKZAB}(\mathrm{K})=\operatorname{RKZ}(\mathrm{K})$

$\operatorname{RKYAB}(\mathrm{K})=\mathrm{RKY}(\mathrm{K})$

CONTINUE

ENDIF

DO 40 K=0,NSOLID

CALL ULMOMF

$\mathrm{RUK}(\mathrm{K}, \mathrm{IJ})=\mathrm{RKR}(\mathrm{K}) * \mathrm{UK}(\mathrm{K}, \mathrm{IJ})+\mathrm{RKRAB}(\mathrm{K}) * \mathrm{GRAVX} * \mathrm{DT}$

-DTOBDR(I)*(ULFR(K)-ULFL(K))

-DTODZ(J)*(ULFT(K)-ULFB(K,I))-DT*SULC(K)

$-1 / \mathrm{R}(\mathrm{I}) * \mathrm{DTODY}(\mathrm{L}) *(\mathrm{ULFA}(\mathrm{K})-\mathrm{ULFF}(\mathrm{K}, \mathrm{I}, \mathrm{J}))$

$\operatorname{ULFL}(\mathrm{K})=\mathrm{ULFR}(\mathrm{K})$

$\operatorname{ULFB}(\mathrm{K}, \mathrm{I})=\operatorname{ULFT}(\mathrm{K})$

$\operatorname{ULFF}(\mathrm{K}, \mathrm{I}, \mathrm{J})=\operatorname{ULFA}(\mathrm{K})$

CALL VLMOMF

$\mathrm{RVK}(\mathrm{K}, \mathrm{IJ})=\mathrm{RKZ}(\mathrm{K}) * \mathrm{VK}(\mathrm{K}, \mathrm{IJ})+\mathrm{RKZAB}(\mathrm{K}) * \mathrm{GRAVY} * \mathrm{DT}$

-DTORDR(I)*(VLFR(K)-VLFL(K))

-DTODZP(J)*(VLFT(K)-VLFB(K,I))

$-1 / \mathrm{R}(\mathrm{I}) * \mathrm{DTODY}(\mathrm{L}) *(\mathrm{VLFA}(\mathrm{K})-\mathrm{VLFF}(\mathrm{K}, \mathrm{I}, \mathrm{J}))$

$\operatorname{VLFL}(\mathrm{K})=\operatorname{VLFR}(\mathrm{K})$

$\operatorname{VLFB}(\mathrm{K}, \mathrm{I})=\operatorname{VLFT}(\mathrm{K})$

$\operatorname{VLFF}(\mathrm{K}, \mathrm{I}, \mathrm{J})=\operatorname{VLFA}(\mathrm{K})$

CALL WLMOMF

$\mathrm{RWK}(\mathrm{K}, \mathrm{IJ})=\mathrm{RKY}(\mathrm{K}) * \mathrm{WK}(\mathrm{K}, \mathrm{IJ})+\mathrm{RKYAB}(\mathrm{K}) * \mathrm{GRAVZ} * \mathrm{DT}$

-DTORDR(I)*(WLFR(K)-WLFL(K))

-DTODZ(J)*(WLFT(K)-WLFB(K,I))

$-1 /$ R(I)*DTODYP(L)*(WLFA(K)-WLFF(K,I,J)) 


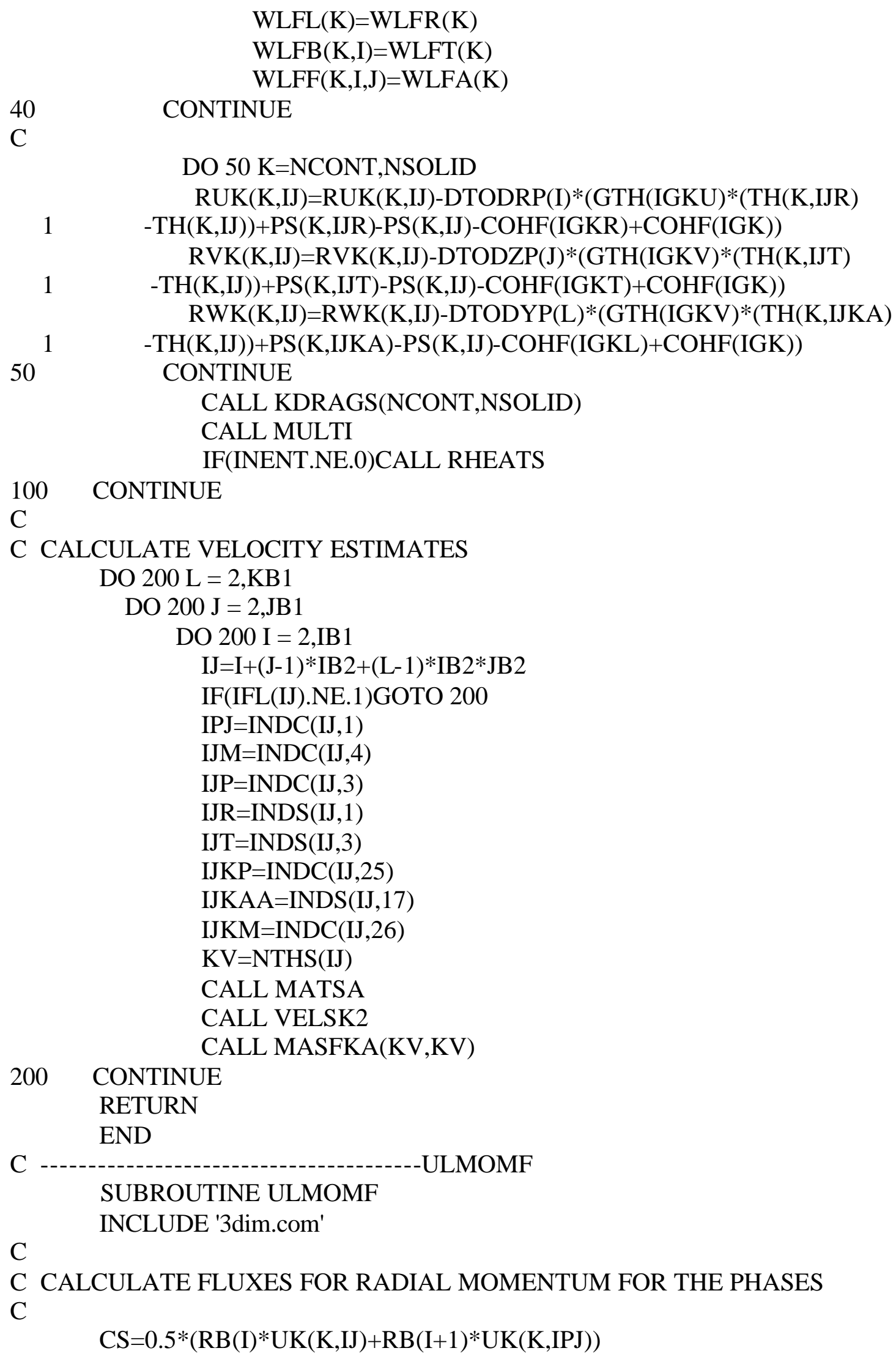




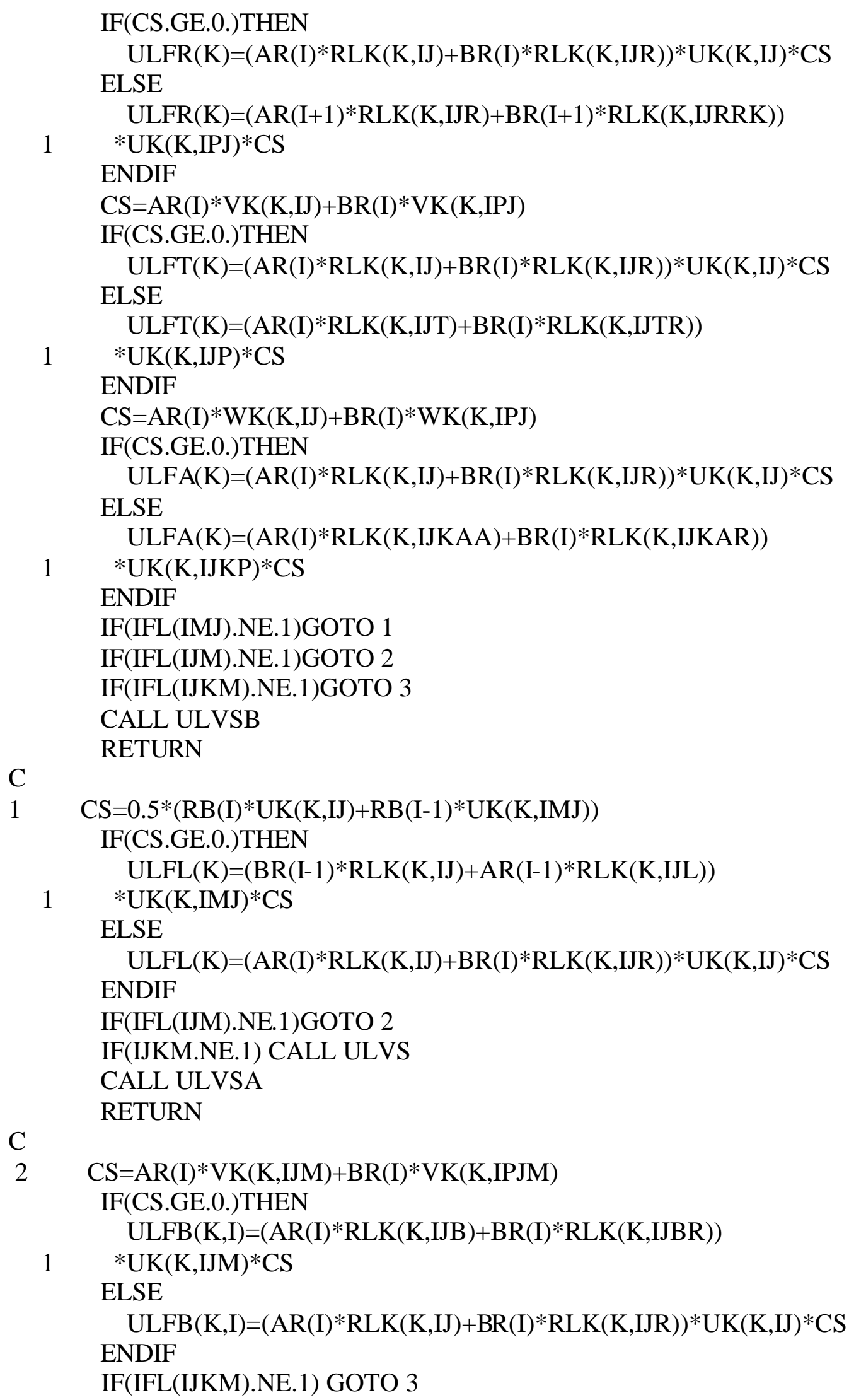




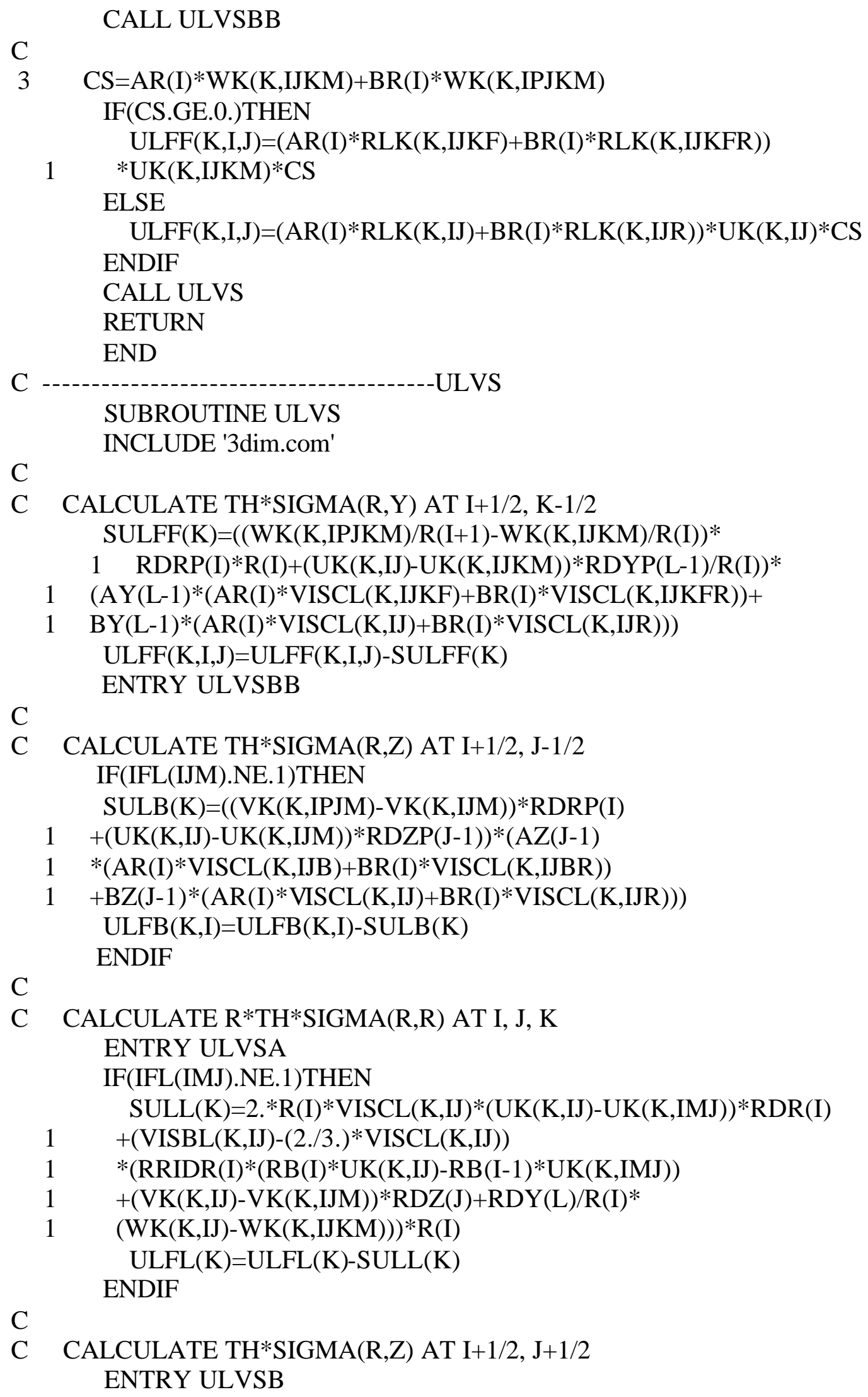




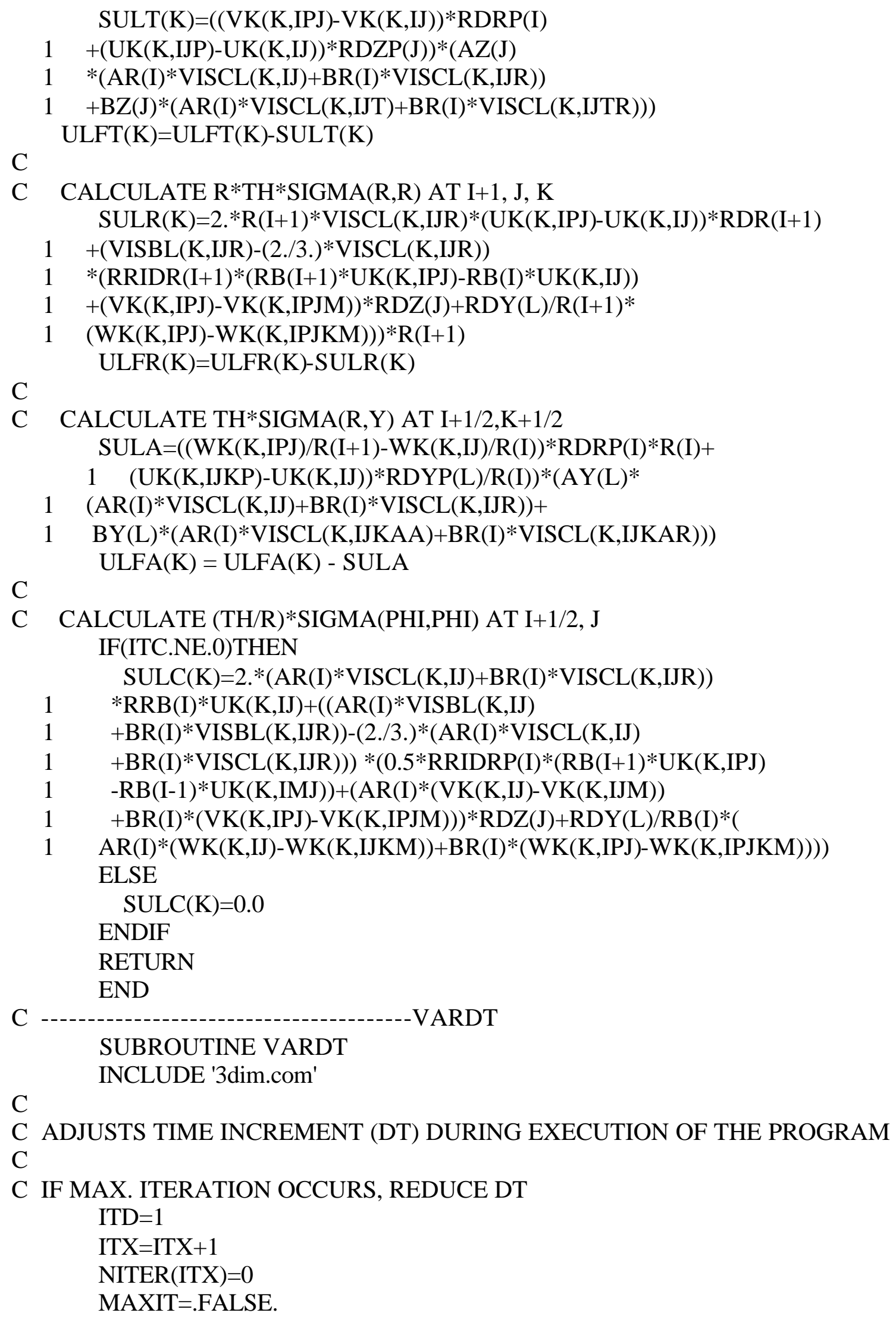


IF(ITX.LE.ITXMX)THEN

DT=DTNXT(ITX)

C READ RESTART FILE TO START AGAIN WITH LOWER DT

REWIND(9)

CALL TAPERD

TDUMP1=TDUMP1-TDUMP

CALL SETUP

ELSE

ITX=ITXMX

ENDIF

GOTO 100

$\mathrm{C}$

ENTRY VARDTI

C IF CONVERGENCE IS ACHIEVED FOR MORE THAN IMX TIMES

C FOR A DT, INCREASE DT

ITX=ITXN

DT=DTNXT(ITX)

ITD $=1$

CALL SETRZ

100 CONTINUE

ITINT $=0$

RETURN

END

C -VELINV

SUBROUTINE VELINV(NS,NP,A,B)

DIMENSION A(NP,NP),B(NP)

C

C USE GAUSS-DOLITTLE METHOD FOR SYMMETRIC MATRIX INVERSION

DO $136 \mathrm{~K}=2, \mathrm{NP}$

IF(ABS(A(K,K)).GE.1.E-6)GOTO 136

DO $135 \mathrm{KK}=1, \mathrm{NP}$

$\mathrm{A}(\mathrm{K}, \mathrm{KK})=0.0$

$135 \quad \mathrm{~A}(\mathrm{KK}, \mathrm{K})=0.0$

$\mathrm{B}(\mathrm{K})=0.0$

136 CONTINUE

C

DO $160 \mathrm{~K}=1, \mathrm{NP}$

IF(A(K,K).EQ.0.0)GOTO 160

$\mathrm{KP} 1=\mathrm{K}+1$

$\mathrm{DIV}=1 . / \mathrm{A}(\mathrm{K}, \mathrm{K})$

DO $140 \mathrm{KJ}=\mathrm{KP} 1$,NP

$140 \quad \mathrm{~A}(\mathrm{~K}, \mathrm{KJ})=\mathrm{A}(\mathrm{K}, \mathrm{KJ}) * \mathrm{DIV}$

$\mathrm{B}(\mathrm{K})=\mathrm{B}(\mathrm{K}) * \mathrm{DIV}$

DO $150 \mathrm{KI}=\mathrm{KP} 1, \mathrm{NP}$

$\mathrm{AMUL}=\mathrm{A}(\mathrm{KI}, \mathrm{K})$

DO $145 \mathrm{KJ}=\mathrm{KP} 1$,NP 


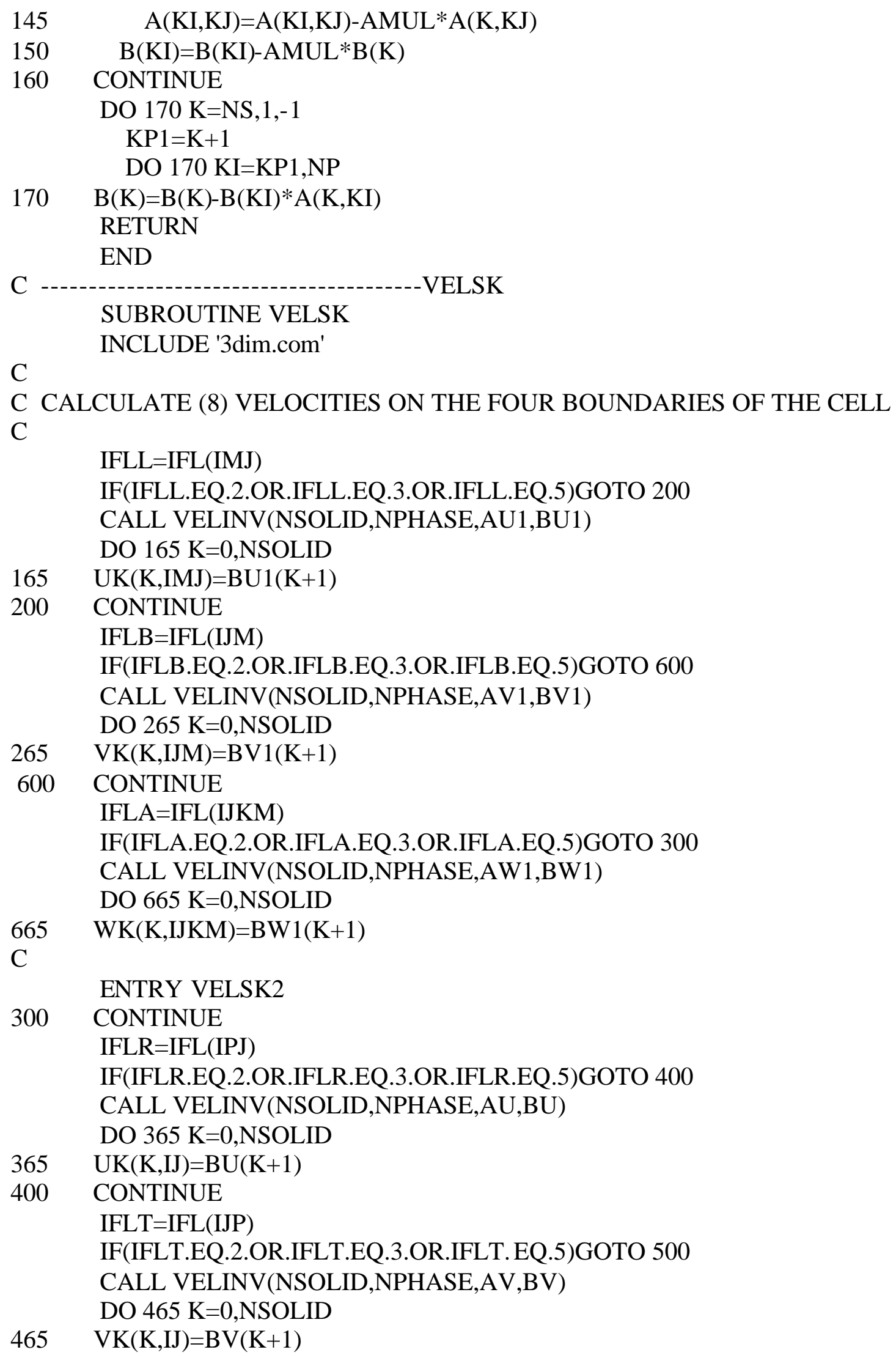


CONTINUE

IFLFF=IFL(IJKP)

IF(IFLFF.EQ.2.OR.IFLFF.EQ.3.OR.IFLFF.EQ.5)RETURN

CALL VELINV(NSOLID,NPHASE,AW,BW)

DO $565 \mathrm{~K}=0$,NSOLID

$565 \mathrm{WK}(\mathrm{K}, \mathrm{IJ})=\mathrm{BW}(\mathrm{K}+1)$

RETURN

END

$\mathrm{C}$

SUBROUTINE VLMOMF

VLMOMF

INCLUDE '3dim.com'

$\mathrm{C}$

C CALCULATES FLUXES OF AXIAL MOMENTUM FOR THE PHASES

$\mathrm{C}$

$\mathrm{CS}=0.5^{*}(\mathrm{VK}(\mathrm{K}, \mathrm{IJ})+\mathrm{VK}(\mathrm{K}, \mathrm{IJP}))$

IF(CS.GE.0.)THEN

$\operatorname{VLFT}(\mathrm{K})=(\mathrm{AZ}(\mathrm{J}) * \mathrm{RLK}(\mathrm{K}, \mathrm{IJ})+\mathrm{BZ}(\mathrm{J}) * \mathrm{RLK}(\mathrm{K}, \mathrm{IJT}))^{*} \mathrm{VK}(\mathrm{K}, \mathrm{IJ}) * \mathrm{CS}$

ELSE

$\operatorname{VLFT}(\mathrm{K})=(\mathrm{AZ}(\mathrm{J}+1) * \mathrm{RLK}(\mathrm{K}, \mathrm{IJT})+\mathrm{BZ}(\mathrm{J}+1) * \mathrm{RLK}(\mathrm{K}, \mathrm{IJTTK}))$

$1 \quad * \mathrm{VK}(\mathrm{K}, \mathrm{IJP}) * \mathrm{CS}$

ENDIF

$\mathrm{CS}=(\mathrm{AZ}(\mathrm{J}) * \mathrm{UK}(\mathrm{K}, \mathrm{IJ})+\mathrm{BZ}(\mathrm{J}) * \mathrm{UK}(\mathrm{K}, \mathrm{IJP}))^{*} \mathrm{RB}(\mathrm{I})$

IF(CS.GE.0.)THEN

$\mathrm{VLFR}(\mathrm{K})=(\mathrm{AZ}(\mathrm{J}) * \mathrm{RLK}(\mathrm{K}, \mathrm{IJ})+\mathrm{BZ}(\mathrm{J}) * \mathrm{RLK}(\mathrm{K}, \mathrm{IJT})) * \mathrm{VK}(\mathrm{K}, \mathrm{IJ}) * \mathrm{CS}$

ELSE

$\operatorname{VLFR}(\mathrm{K})=(\mathrm{AZ}(\mathrm{J}) * \mathrm{RLK}(\mathrm{K}, \mathrm{IJR})+\mathrm{BZ}(\mathrm{J}) * \mathrm{RLK}(\mathrm{K}, \mathrm{IJTR}))$

$1 \quad * \mathrm{VK}(\mathrm{K}, \mathrm{IPJ}) * \mathrm{CS}$

ENDIF

$\mathrm{CS}=(\mathrm{AZ}(\mathrm{J}) * \mathrm{WK}(\mathrm{K}, \mathrm{IJ})+\mathrm{BZ}(\mathrm{J}) * \mathrm{WK}(\mathrm{K}, \mathrm{IJP}))$

IF(CS.GE.0.)THEN

$\mathrm{VLFA}(\mathrm{K})=(\mathrm{AZ}(\mathrm{J}) * \mathrm{RLK}(\mathrm{K}, \mathrm{IJ})+\mathrm{BZ}(\mathrm{J}) * \mathrm{RLK}(\mathrm{K}, \mathrm{IJT})) * \mathrm{VK}(\mathrm{K}, \mathrm{IJ}) * \mathrm{CS}$

ELSE

$\operatorname{VLFA}(\mathrm{K})=(\mathrm{AZ}(\mathrm{J}) * \mathrm{RLK}(\mathrm{K}, \mathrm{IJKAA})+\mathrm{BZ}(\mathrm{J}) * \mathrm{RLK}(\mathrm{K}, \mathrm{IJKAT}))$

$1 \quad * \mathrm{VK}(\mathrm{K}, \mathrm{IJKP}) * \mathrm{CS}$

ENDIF

IF(IFL(IMJ).NE.1)GOTO 1

IF(IFL(IJM).NE.1)GOTO 2

IF(IFL(IJKM).NE.1) GOTO 3

CALL VLVSB

RETURN

$\mathrm{C}$

$1 \mathrm{CS}=(\mathrm{AZ}(\mathrm{J}) * \mathrm{UK}(\mathrm{K}, \mathrm{IMJ})+\mathrm{BZ}(\mathrm{J}) * \mathrm{UK}(\mathrm{K}, \mathrm{IMJP})) * \mathrm{RB}(\mathrm{I}-1)$

IF(CS.GE.0.)THEN

$\operatorname{VLFL}(\mathrm{K})=(\mathrm{AZ}(\mathrm{J}) * \mathrm{RLK}(\mathrm{K}, \mathrm{IJL})+\mathrm{BZ}(\mathrm{J}) * \mathrm{RLK}(\mathrm{K}, \mathrm{IJTL}))$

$1 \quad * \mathrm{VK}(\mathrm{K}, \mathrm{IMJ}) * \mathrm{CS}$

ELSE 


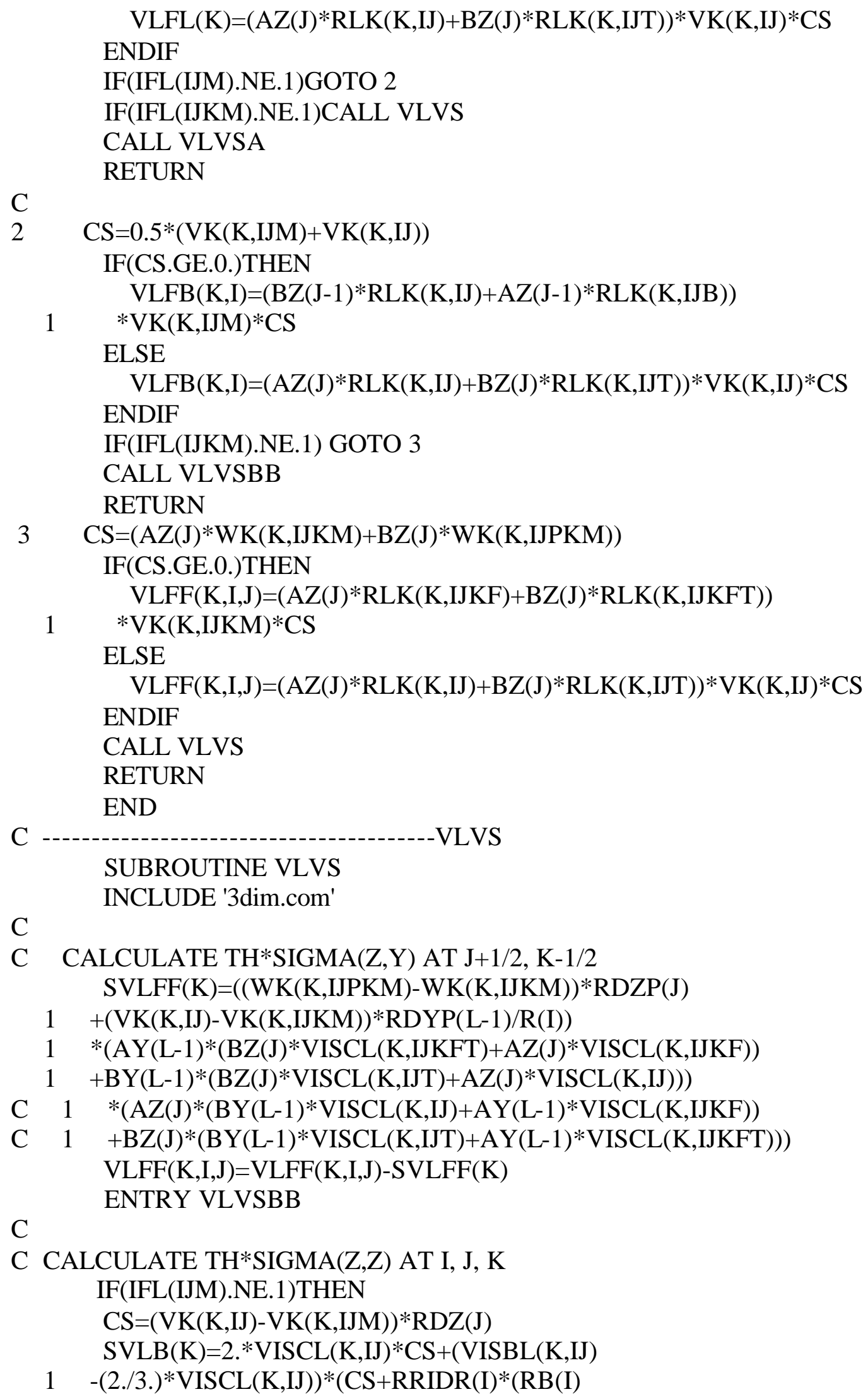




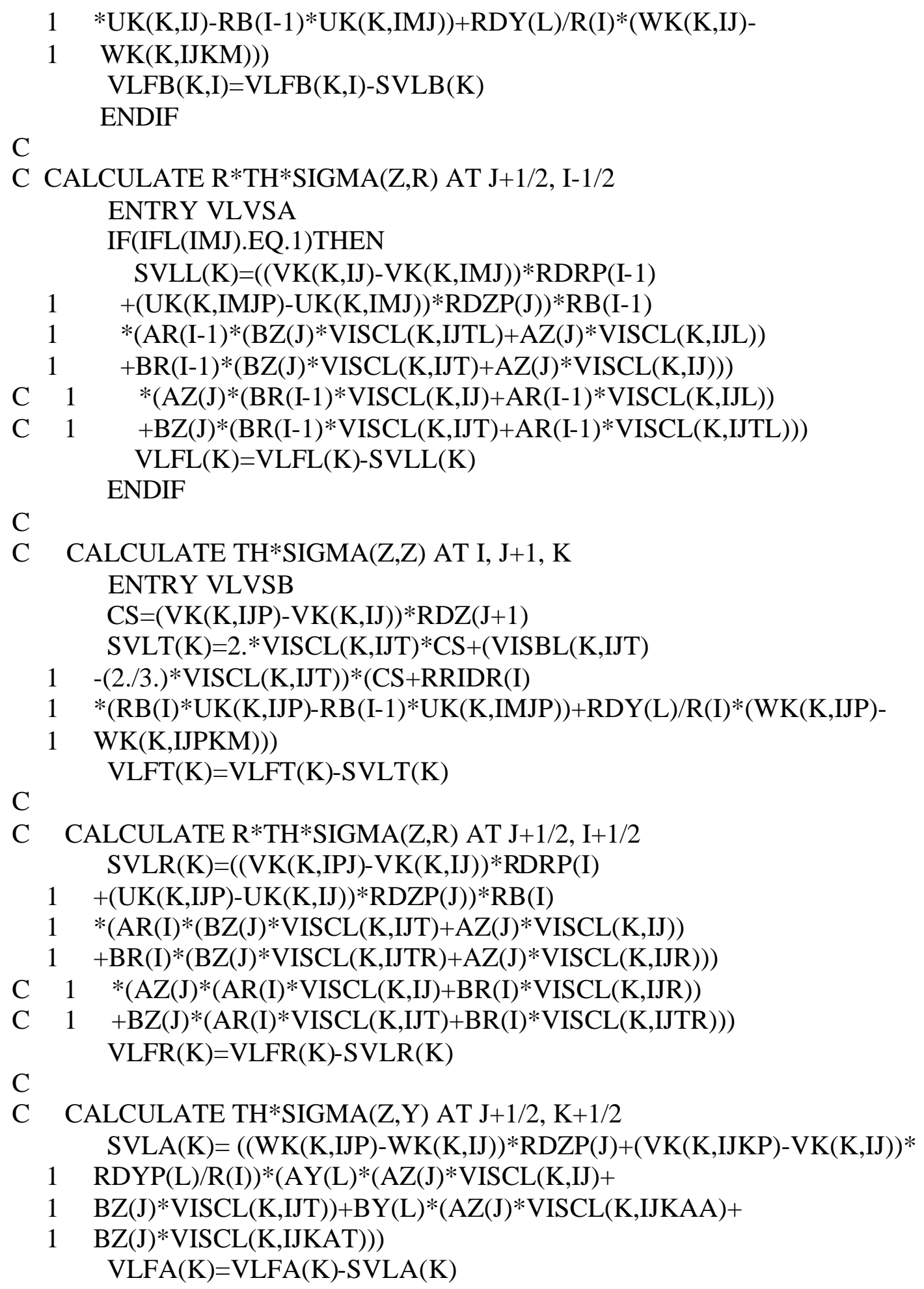
VRELS 
SUBROUTINE VRELS

INCLUDE '3dim.com'

$\mathrm{C}$

C CALCULATE SQUARE OF RELATIVE VELOCITY BETWEEN FIELDS

DO $10 \mathrm{~K}=0$, NSOLID

IF(K.NE.KV)THEN

$\mathrm{DV}=0.5 *(\mathrm{VK}(\mathrm{KV}, \mathrm{IJ})-\mathrm{VK}(\mathrm{K}, \mathrm{IJ})+\mathrm{VK}(\mathrm{KV}, \mathrm{IJM})-\mathrm{VK}(\mathrm{K}, \mathrm{IJM}))$

$\mathrm{DW}=0.5^{*}(\mathrm{WK}(\mathrm{KV}, \mathrm{IJ})-\mathrm{WK}(\mathrm{K}, \mathrm{IJ})+\mathrm{WK}(\mathrm{KV}, \mathrm{IJKM})-\mathrm{WK}(\mathrm{K}, \mathrm{IJKM}))$

$\mathrm{DU}=0.5 *(\mathrm{RB}(\mathrm{I}) *(\mathrm{UK}(\mathrm{KV}, \mathrm{IJ})-\mathrm{UK}(\mathrm{K}, \mathrm{IJ}))$

$\left.1+\mathrm{RB}(\mathrm{I}-1)^{*}(\mathrm{UK}(\mathrm{KV}, \mathrm{IMJ})-\mathrm{UK}(\mathrm{K}, \mathrm{IMJ}))\right) / \mathrm{R}(\mathrm{I})$

$\operatorname{SVREL}(\mathrm{K})=\mathrm{DU} * \mathrm{DU}+\mathrm{DV} * \mathrm{DV}+\mathrm{DW} * \mathrm{DW}$

ENDIF

10 CONTINUE

RETURN

END

$\mathrm{C}$

SUBROUTINE VWORKL(NPH1,NPH2)

INCLUDE '3dim.com'

$\mathrm{C}$

C CALCULATE VISCOUS STRESSES FOR THE WORK TERM IN THE PHASES

C ENERGY EQUATION (NOTE: VISCOSITY IS MULTIPLIED LATER)

$\mathrm{C}$

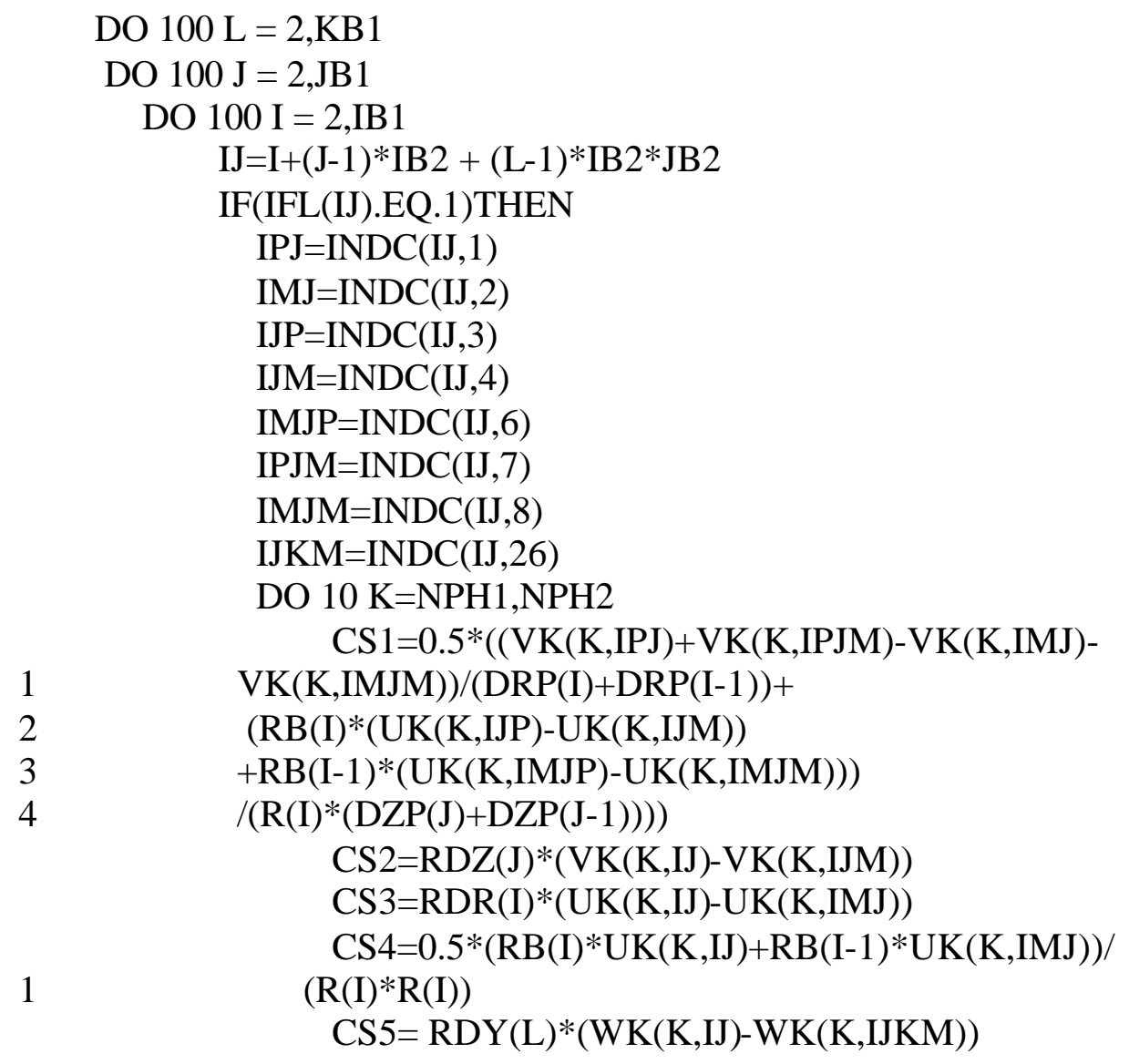


$\mathrm{C}$

$\operatorname{SILM}(\mathrm{K}, \mathrm{IJ})=\mathrm{RRIDR}(\mathrm{I}) *(\mathrm{RB}(\mathrm{I}) * \mathrm{UK}(\mathrm{K}, \mathrm{IJ})$

$-\mathrm{RB}(\mathrm{I}-1) * \mathrm{UK}(\mathrm{K}, \mathrm{IMJ}))+\mathrm{CS} 2+\mathrm{CS} 5$

SILRZ $=$ CS1

SILZZ $=2.0 * \mathrm{CS} 2$

SILRR $=2.0 * \mathrm{CS} 3$

SILPP $=2.0 *$ CS4

SILPY $=2 *$ CS5

$\mathrm{C}$

C CALCULATE VISCOUS WORK FOR THE PHASES

$\operatorname{VWLS}(\mathrm{K}, \mathrm{IJ})=\mathrm{DT} *(\mathrm{SILRZ} * \mathrm{CS} 1+\mathrm{SILZZ} * \mathrm{CS} 2+\mathrm{SILRR} * \mathrm{CS} 3$

$1+$ SILPY*CS5+SILPP*CS4*ITC)

$\operatorname{VWLM}(\mathrm{K}, \mathrm{IJ})=\mathrm{DT} * \operatorname{SILM}(\mathrm{K}, \mathrm{IJ}) * \operatorname{SILM}(\mathrm{K}, \mathrm{IJ})$

10 CONTINUE

100 CONTINUE

RETURN

END

$\mathrm{C}$ WLMOMF

SUBROUTINE WLMOMF

INCLUDE '3dim.com'

$\mathrm{C}$

C CALCULATES FLUXES OF THE 3rd DIR. MOMENTUM FOR THE PHASES

$\mathrm{C}$

$\mathrm{CS}=\mathrm{AY}(\mathrm{L}) * \mathrm{VK}(\mathrm{K}, \mathrm{IJ})+\mathrm{BY}(\mathrm{L}) * \mathrm{VK}(\mathrm{K}, \mathrm{IJKP})$

IF(CS.GE.0.)THEN

$\mathrm{WLFT}(\mathrm{K})=(\mathrm{AY}(\mathrm{L}) * \mathrm{RLK}(\mathrm{K}, \mathrm{IJ})+\mathrm{BY}(\mathrm{L}) * \mathrm{RLK}(\mathrm{K}, \mathrm{IJKAA})) * \mathrm{WK}(\mathrm{K}, \mathrm{IJ}) * \mathrm{CS}$

ELSE

$\operatorname{WLFT}(\mathrm{K})=(\mathrm{AY}(\mathrm{L}) * \mathrm{RLK}(\mathrm{K}, \mathrm{IJT})+\mathrm{BY}(\mathrm{L}) * \mathrm{RLK}(\mathrm{K}, \mathrm{IJKAT}))$

$1 \quad * \mathrm{WK}(\mathrm{K}, \mathrm{IJP}) * \mathrm{CS}$

ENDIF

$\mathrm{CS}=(\mathrm{AY}(\mathrm{L}) * \mathrm{UK}(\mathrm{K}, \mathrm{IJ})+\mathrm{BY}(\mathrm{L}) * \mathrm{UK}(\mathrm{K}, \mathrm{IJKP})) * \mathrm{RB}(\mathrm{I})$

IF(CS.GE.0.)THEN

$\mathrm{WLFR}(\mathrm{K})=(\mathrm{AY}(\mathrm{L}) * \mathrm{RLK}(\mathrm{K}, \mathrm{IJ})+\mathrm{BY}(\mathrm{L}) * \mathrm{RLK}(\mathrm{K}, \mathrm{IJKAA})) * \mathrm{WK}(\mathrm{K}, \mathrm{IJ}) * \mathrm{CS}$ ELSE

$\operatorname{WLFR}(\mathrm{K})=(\mathrm{AY}(\mathrm{L}) * \mathrm{RLK}(\mathrm{K}, \mathrm{IJR})+\mathrm{BY}(\mathrm{L}) * \mathrm{RLK}(\mathrm{K}, \mathrm{IJKAR}))$

$1 \quad * \mathrm{WK}(\mathrm{K}, \mathrm{IPJ}) * \mathrm{CS}$

ENDIF

$\mathrm{CS}=0.5^{*}(\mathrm{WK}(\mathrm{K}, \mathrm{IJ})+\mathrm{WK}(\mathrm{K}, \mathrm{IJKP}))$

IF(CS.GE.0.)THEN

$\mathrm{WLFA}(\mathrm{K})=(\mathrm{AY}(\mathrm{L}) * \mathrm{RLK}(\mathrm{K}, \mathrm{IJ})+\mathrm{BY}(\mathrm{L}) * \mathrm{RLK}(\mathrm{K}, \mathrm{IJKAA}))^{*} \mathrm{WK}(\mathrm{K}, \mathrm{IJ}) * \mathrm{CS}$

ELSE

$\operatorname{WLFA}(\mathrm{K})=(\mathrm{AY}(\mathrm{L}+1) * \mathrm{RLK}(\mathrm{K}, \mathrm{IJKAA})+\mathrm{BY}(\mathrm{L}+1) * \mathrm{RLK}(\mathrm{K}, \mathrm{IJKA}))$

$1 \quad * \mathrm{WK}(\mathrm{K}, \mathrm{IJKP}) * \mathrm{CS}$

ENDIF

IF(IFL(IMJ).NE.1)GOTO 1 


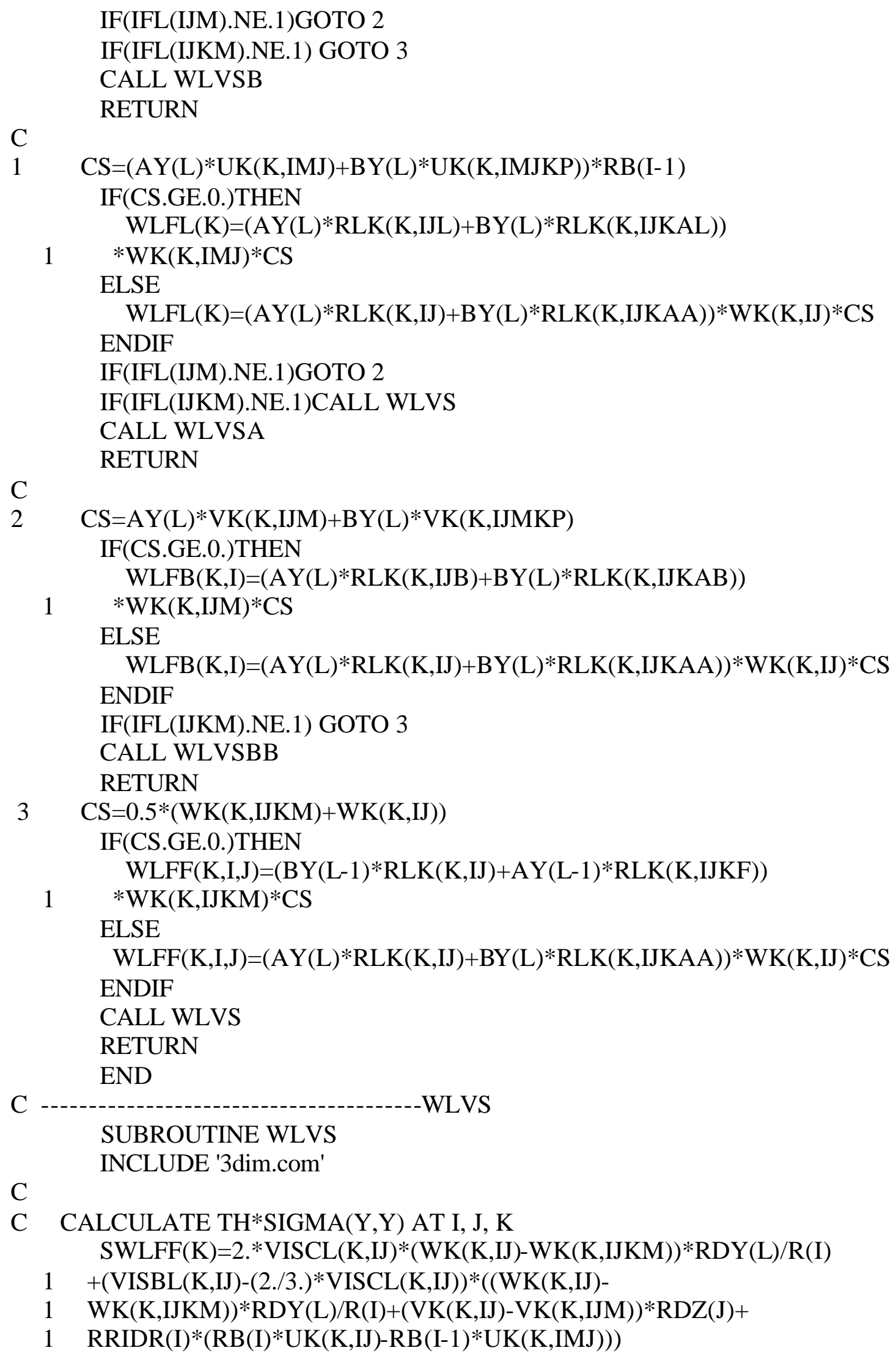




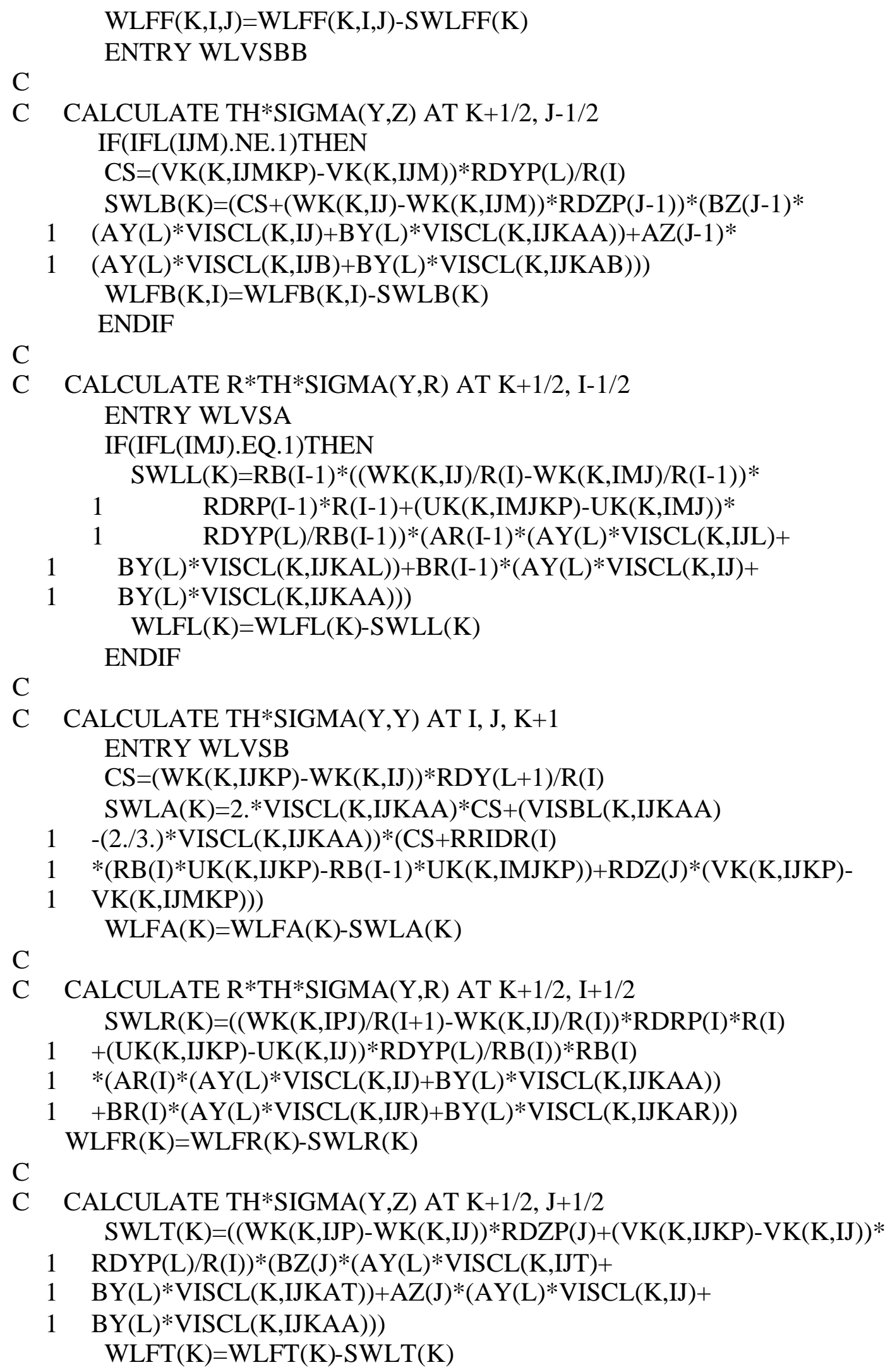




\section{RETURN}

END

\section{Sample Input Data}

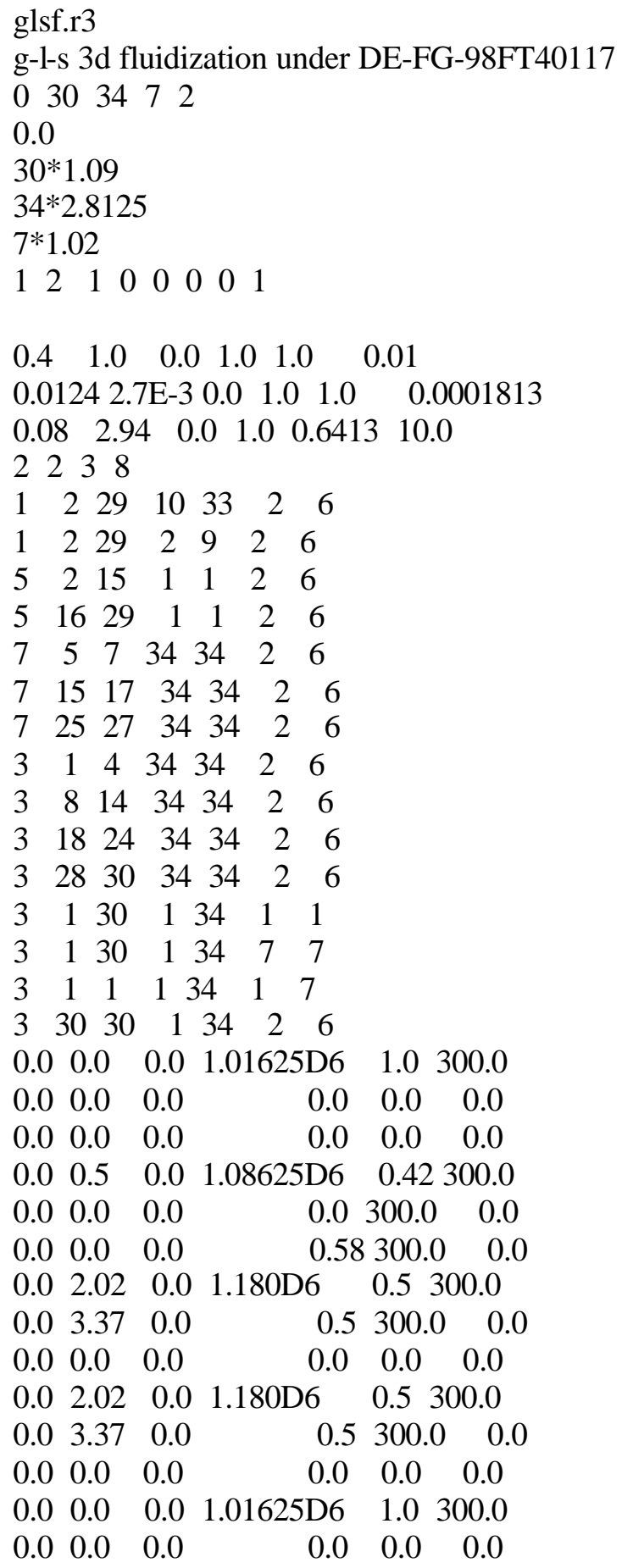




$\begin{array}{lrrrrr}0.0 & 0.0 & 0.0 & 0.0 & 0.0 & 0.0 \\ 0.0 & 0.0 & 0.0 & 1.01625 \mathrm{D} 6 & 1.0 & 300.0 \\ 0.0 & 0.0 & 0.0 & 0.0 & 0.0 & 0.0 \\ 0.0 & 0.0 & 0.0 & 0.0 & 0.0 & 0.0 \\ 0.0 & 0.0 & 0.0 & 1.01625 \mathrm{D} 6 & 1.0 & 300.0 \\ 0.0 & 0.0 & 0.0 & 0.0 & 0.0 & 0.0 \\ 0.0 & 0.0 & 0.0 & 0.0 & 0.0 & 0.0 \\ 0 & & & & \\ 0.0 & 100 & 0.000005 & 1 & & \\ 0.1 & 0.1 & & & \\ 1 & 0.000005 & & & \\ 0.0 & -980.621 & 0.0 \\ 0.999 & 30.48 & 1 . \mathrm{E}-5 & 0.1 & 1 . \mathrm{E}-5\end{array}$

